\title{
The interaction between Hedgehog/Patched and Ras signaling in Rhabdomyosarcoma
}

\section{Dissertation}

\author{
In partial fulfillment of the requirements for the degree \\ "Doctor rerum naturalium (Dr. rer. nat.)" \\ within the Molecular Medicine Study Program \\ of the Georg-August University School of Science (GAUSS)
}

submitted by

Nicole Cuvelier

born in Mechernich

Göttingen 2016 

Members of the Thesis Committee

Prof. Dr. Heidi Hahn

Dept. of Human Genetics; University Medical Center Göttingen

Prof. Dr. Dieter Kube

Dept. of Hematology and Medical Oncology; Georg-August-University Göttingen

Prof. Dr. Felix Brembeck

\section{Members of the Examination Board}

Referee: Prof. Dr. Heidi Hahn

Dept. of Human Genetics; Georg-August-University Göttingen

Co- referee: Prof. Dr. Dieter Kube

Dept. of Hematology and Medical Oncology; Georg-August-University Göttingen

Further members of the Examination Board

Prof. Dr. Felix Brembeck

Prof. Dr. Matthias Dobbelstein

Dept. of Molecular Oncology; Georg-August-University Göttingen

Prof. Dr. Hubertus Jarry

Dept. of Research; University Medical Center Göttingen

Prof. Dr. Christof Kramm

Dept. of Pediatric Hamatologie and Oncologie; University Medical Center Göttingen

Date of Disputation: 7.03.2016 


\section{Affidavit}

I hereby declare that the doctoral thesis entitled "The interaction between Hedgehog/Patched and Ras signaling in Rhabdomyosarcoma" has been written independently and with no other sources and aids than quoted.

\section{Nicole Cuvelier}

January, 2016 Göttingen 


\section{Content}

Affidavit..................................................................................................................................

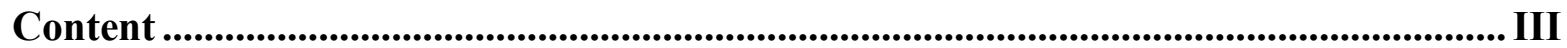

List of Figures .................................................................................................................................. VII

List of Tables.................................................................................................................................. IX

1. Summary ….............................................................................................................................

2. Introduction .........................................................................................................................2

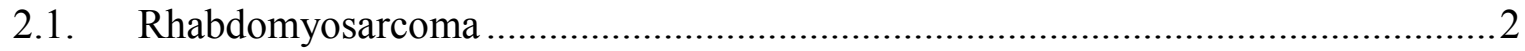

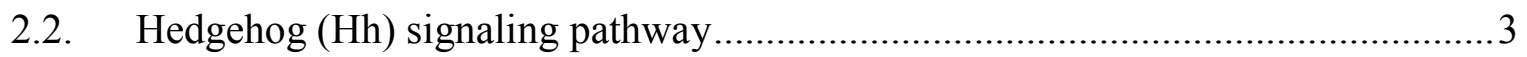

2.3. Hh signaling pathway and RMS ……………………..................................

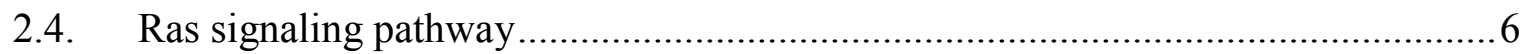

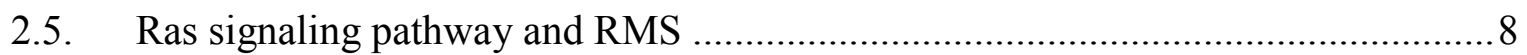

2.6. Interaction between $\mathrm{Hh}$ and Ras signaling ............................................................

2.7. Interaction between Hh and Ras signaling in RMS ............................................11

2.8. Mouse models used in this study.......................................................................

2.8.1. Ptch deficient mice: A mouse model to study the pathogenesis of ERMS ....12

2.8.2. Conditional Ras Mice: Tools for the modulation of oncogenic Ras signaling

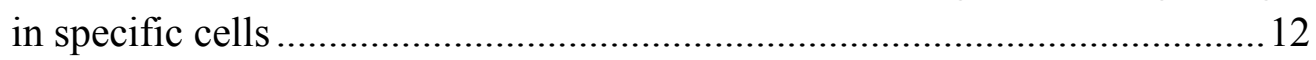

2.8.3. Myf5creERT mice: Tool for induction of conditional mutations in Myf5 expressing cells ....................................................................................... 13

3. Aim of the studies ..................................................................................................................14

4. Materials and Methods ..........................................................................................15

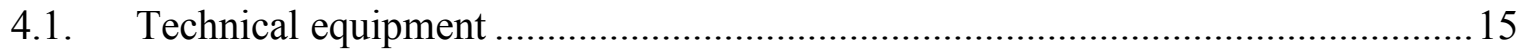

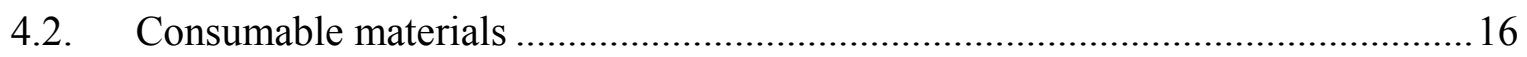

4.3. Reagents and Chemicals............................................................................... 18

4.4. Kits and ready-to-use reaction systems ………………..................................... 19

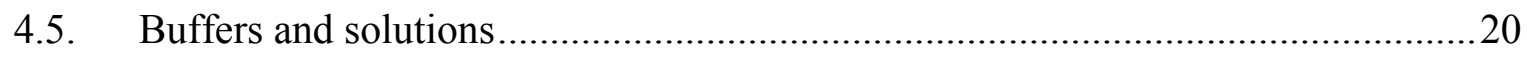

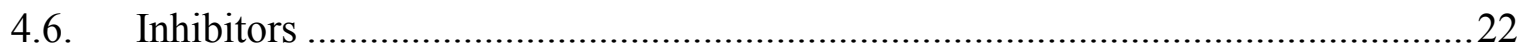

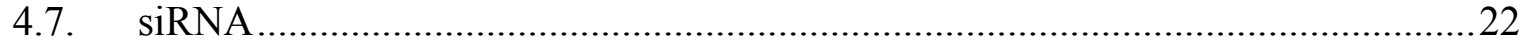

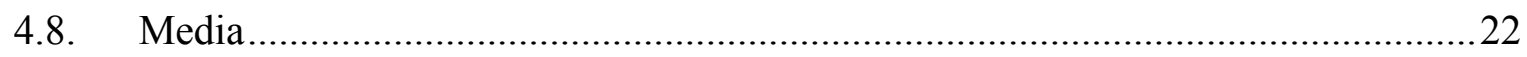

4.8.1. Media and agar plates for bacterial culturing ..............................................2.

4.8.2. Media and reagents for cultivation of eukaryotic cell lines ..........................23 


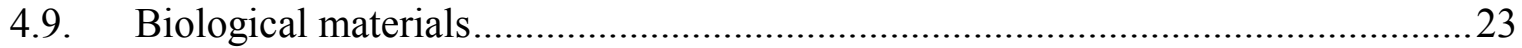

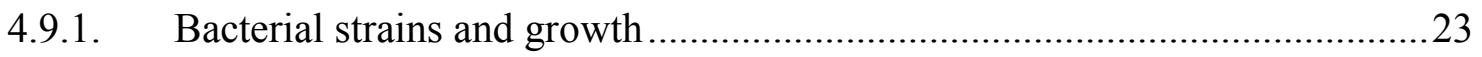

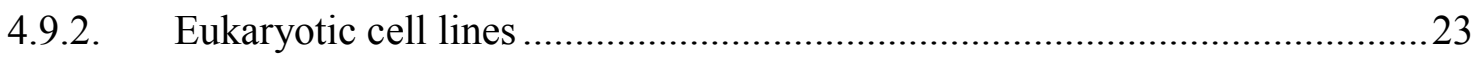

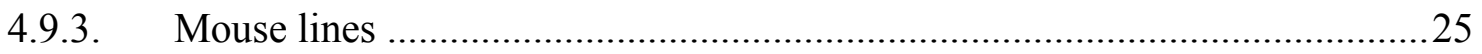

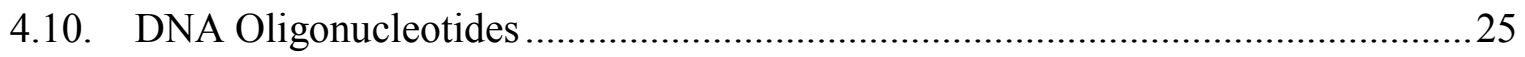

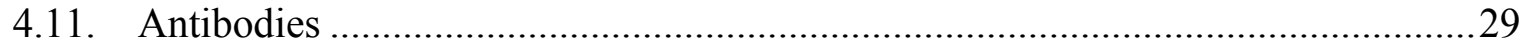

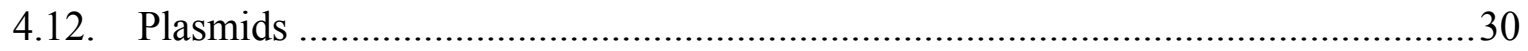

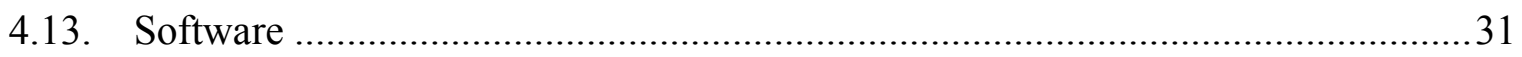

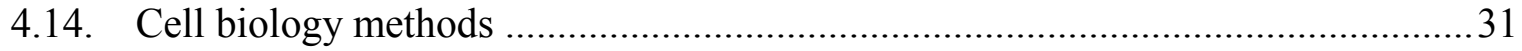

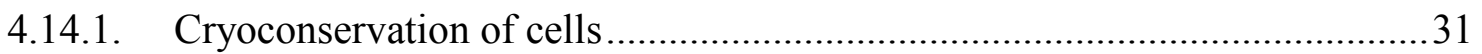

4.14.2. Stable retroviral transduction of RMS cell lines ............................................ 31

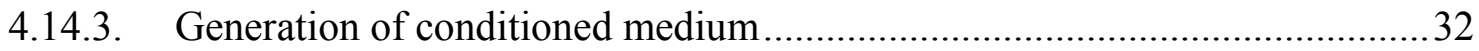

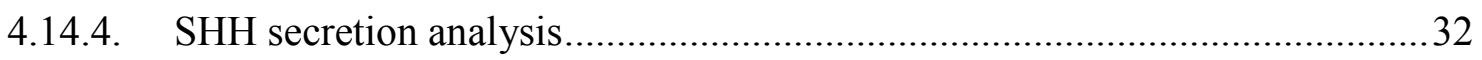

4.14.5. Metabolic activity assay and cell proliferation assay ....................................33

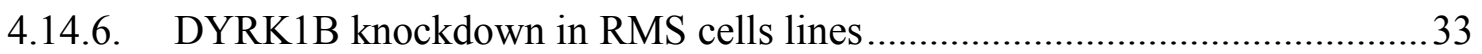

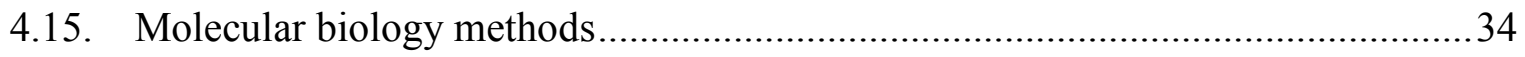

4.15.1. Genomic DNA isolation from murine mouse tail and tissue .........................34

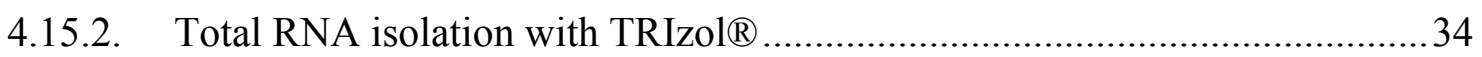

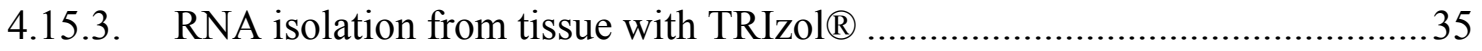

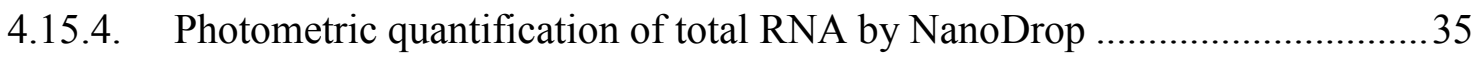

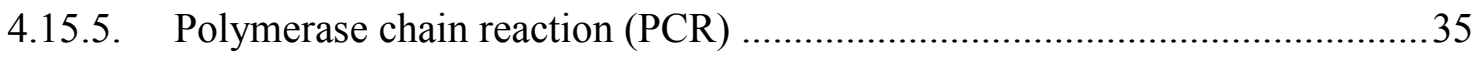

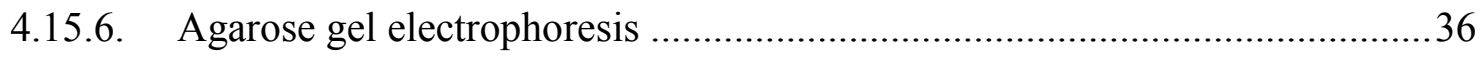

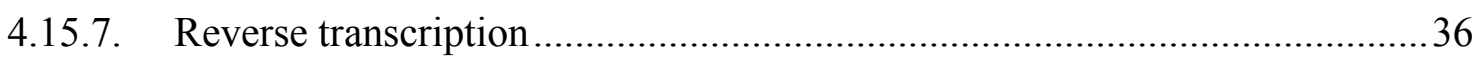

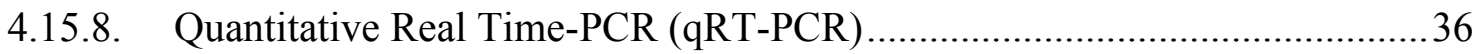

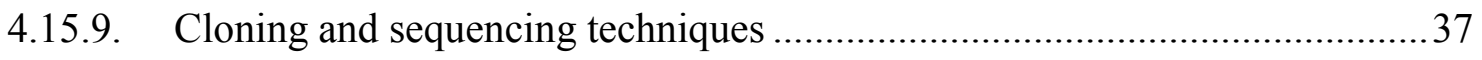

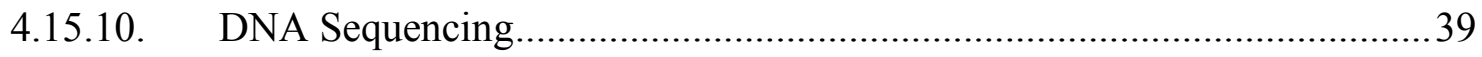

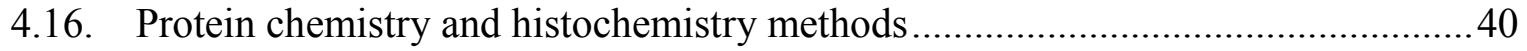

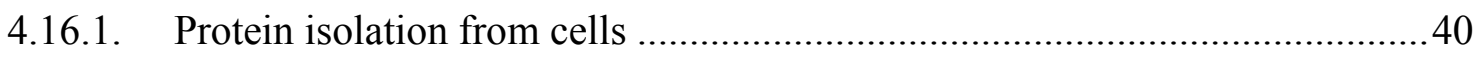

4.16.2. Protein isolation from tissue for Western Blot.......................................... 40

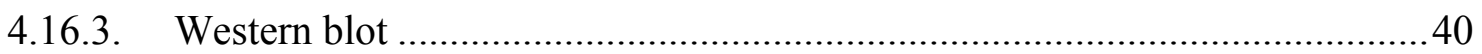

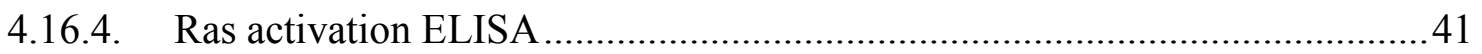

4.16.5. Hematoxylin-Eosin (H\&E) staining of paraffin embedded tissue .................. 41

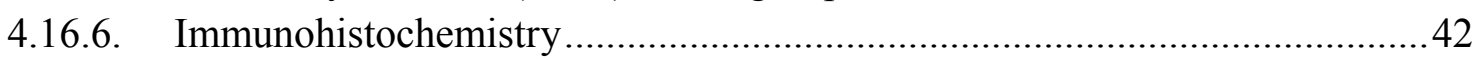

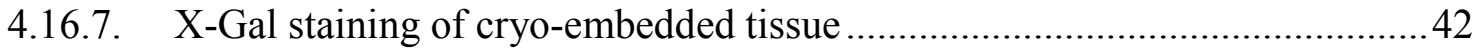

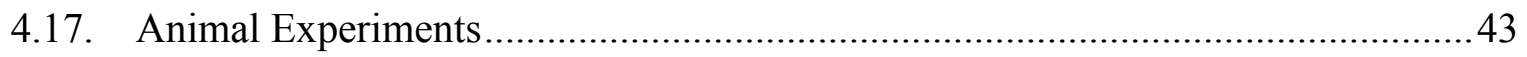

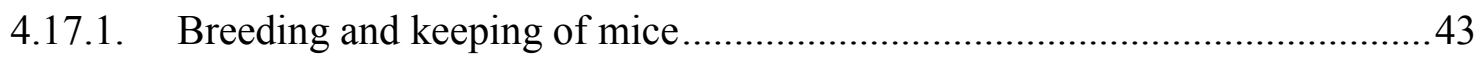

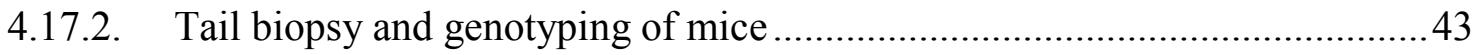

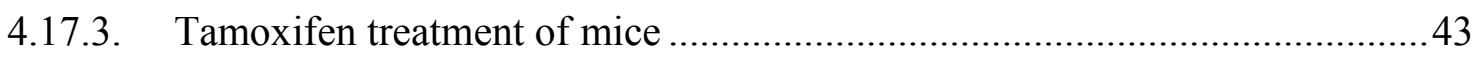

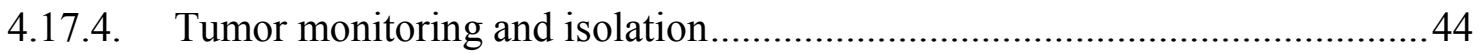

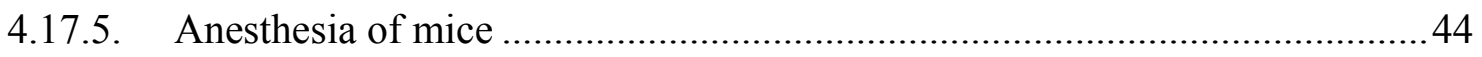

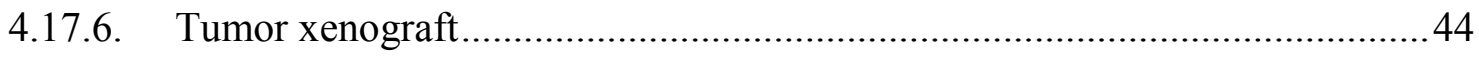


5. Results

5.1. Effects of oncogenic N-, K- or HRAS (in short oncRAS) on human RMS cell lines .46

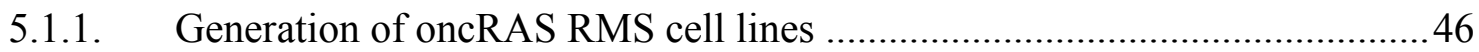

5.1.2. Successful transduction of oncRas in RMS cell lines ....................................... 46

5.1.3. Increased RAS expression levels in oncRas transduced RMS cell lines .......47

5.1.4. No obvious morphological changes of oncRAS transduced RMS cell lines . 48

5.1.5. Inhibition of GLII expression in oncRAS transduced RMS cell lines ..........49

5.1.6. Inhibition of GLII expression in oncRAS transduced RUCH-2 depends on MEK activity ......................................................................................5 52

5.1.7. oncRAS increase pERK levels in RMS-13 and RAS activity in RUCH-2 ....54

5.1.8. HhA decreases and increases pAKT and pERK levels, respectively in RUCH2 cells, whereas UO126 decreases pERK and concomitantly induces pAKT

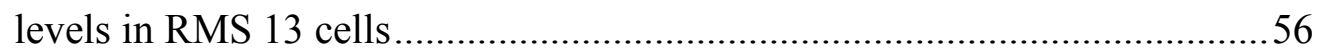

5.1.9. MEK activity inhibits proliferation of oncRAS-transduced RUCH-2 cells ... 57

5.1.10. oncRAS slightly induces muscle differentiation in RMS-13 cells .................61

5.1.11. oncRAS-induced HH signaling inhibition in RMS-13 cells is not mediated by

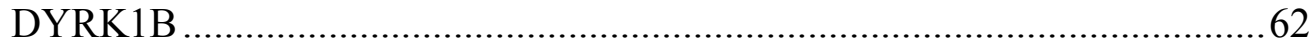

5.2. Growth of oncRas-transduced RMS cell lines in nude mice ...............................64

5.2.1. oncRAS change the tumor growth behavior of RMS tumor xenografts ........64

5.2.2. oncRAS does not obviously influence the morphology of RMS xenografts . 66

5.3. Growth of RMS after conditional expression of oncRas in Ptch mutant mice ...... 67

5.3.1. Generation and identification of $\operatorname{Ptch}^{\text {del/+ }}$ oncRas $^{f l /-} M y f 5 \mathrm{creERT}^{t g /-}$ mice........67

5.3.2. Characterization of RMS after conditional expression of oncNRas in Ptch mutant mice ............................................................................ 75

5.3.3. Characterization of RMS after conditional expression of oncKRas in Ptch

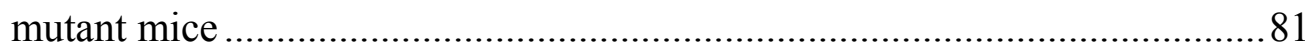

5.3.4. Preliminary characterization of RMS after conditional expression of oncHRas

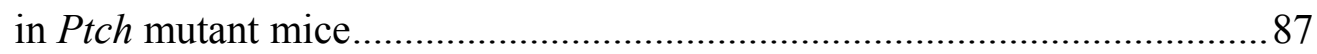

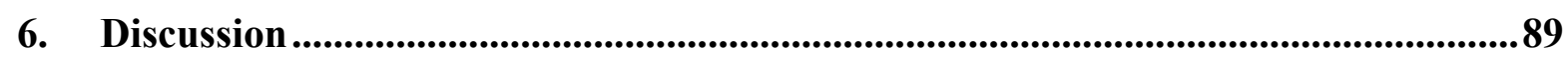

6.1. Generation of RMS models with active Ras signaling in vivo and in vitro ...........89

6.2. No obvious morphological changes of RMS by oncRas....................................90

6.3. Crosstalk of Ras and Hh signaling in RMS ....................................................... 91

6.4. Active Ras signaling changes the growth behavior in RMS ..............................95

6.5. Active Ras signaling slightly modulates the muscle differentiation behavior in RMS.

8. Abbreviations 
Content

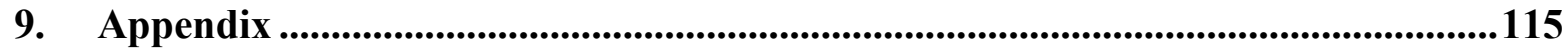

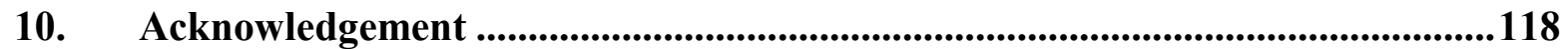

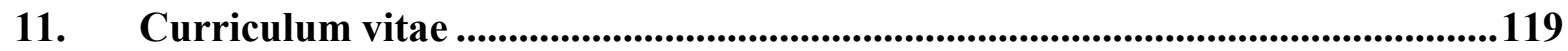




\section{List of Figures}

Figure 1: Simplified schematic drawing of the canonical Hh/Ptch signaling pathway.. ......... 4

Figure 2: Schematic drawing possible interactions between RAS and GLI activation.......... 10

Figure 3: Experimental setting for the SHH secretion analysis. ........................................ 33

Figure 4: PCR Fragments amplified with pMSCV-seq-F and either pNRasID-Rev, pKRasID-Rev or pHRasID-Rev from cDNA isolated from RUCH-2 cells.........................46

Figure 5: Sequences of the PCR fragments shown in Figure 4 ............................................47

Figure 6: Elevated RAS levels in RMS cell lines transduced with oncRAS. ......................48

Figure 7: Morphology of oncRAS transduced RMS cell lines. .........................................49

Figure 8: Downregulation of GLI1 expression in oncRAS transduced RMS cell lines.........50

Figure 9: $S H H$ expression levels in oncRAS transduced RMS cell lines. .............................50

Figure 10: oncRAS transduced RUCH-2 cell lines are not able to secrete SHH..................51

Figure 11: Schematic representation: Treatment strategy of stably transduced RMS cells...52

Figure 12: oncRAS may inhibit GLI1 expression via the MEK/ERK axis in RUCH-2, but

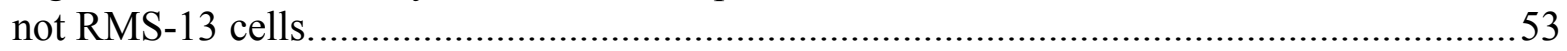

Figure 13: Modulation of RAS effector pathways in oncRAS-transduced RUCH-2 cells....55

Figure 14: Modulation of RAS effector pathways in oncRAS-transduced RMS-13 cells.....55

Figure 15: Moderately increased RAS activity in RUCH-2 cells stably transduced with oncRAS.

Figure 16: Modulation of the proliferative capacity and metabolic activity in oncRAS

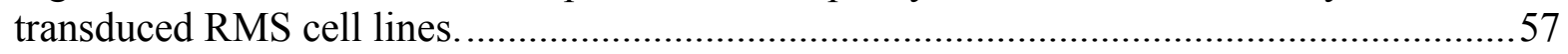

Figure 17: oncRAS modulates the proliferative capacity and metabolic activity ERMS......59

Figure 18: oncRAS modulates the proliferative capacity and metabolic activity in ARMS. 60

Figure 19: Slightly increased muscle differentiation in oncRAS-transduced RMS-13 cells. 61

Figure 20: Downregulation of DYRK1B expression in oncRAS-transduced RMS-13 cells..62

Figure 21: DYRK1B knockdown decreases the expression levels of GLI1 and DYRK1B in oncRAS-transduced RMS-13 cells.

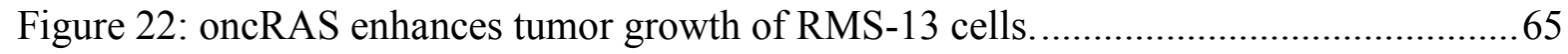

Figure 23: Histology of oncRAS-transduced RMS-13 xenografts. ....................................66

Figure 24: Scheme of the Ptch locus and genotyping of heterozygous Ptch $^{\text {del }}$ mice. 67 
Figure 25: Scheme of the Ras loci and genotyping of conditional oncogenic Ras mice.......68

Figure 26: Scheme of the Myf5 locus and genotyping of the inducible Myf5CreERT mice.. 70

Figure 27: Schematic representation of the schedule for mouse monitoring. .70

Figure 28: Tamoxifen-induced activity of the Myf5creERT in R26R-reporter mice. .71

Figure 29: Recombination at the Ras loci in tamoxifen induced oncNRas and oncKRas Ptch $^{\text {del/+ }}$ oncRas $^{\text {fl/ }}$ Myf5CreERT ${ }^{t g /+}$ mice.

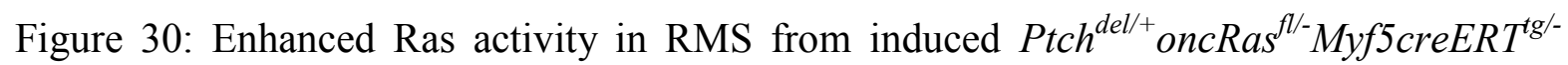
mice.

Figure 31: No significant effect of tamoxifen on RMS incidence, multiplicity and latency time. .75

Figure 32: Increased expression of Gli2 and Gli3 by oncNRas in Ptch mutant RMS. .76

Figure 33: No obvious phosphorylation changes in oncNRas-expressing Ptch mutant RMS.

Figure 34: Decreased expression of MyoD and Myogenin in oncNRas-expressing Ptch mutant RMS.

Figure 35: oncNRas decreases multiplicity of RMS of Ptch mutant mice. .79

Figure 36: Decreased proliferation of oncNRas expressing RMS of Ptch mutant mice........ 80

Figure 37: oncNRas expression does not change the histology of Ptch mutant RMS.

Figure 38: oncKRas decreases the expression of Glis in RMS of Ptch mutant mice.

Figure 39: oncKRas increases pErk and pAkt levels in RMS of Ptch mutant mice.

Figure 40: Slightly increased expression of the myogenesis proliferation and determination markers and decreased muscle differentiation marker in oncRas RMS of Ptch mutant mice.

Figure 41: oncKRas significantly increases tumor incidence and shortens latency time.

Figure 42: oncKRas does not significantly increase the proliferation rate of RMS of Ptch mutant mice.

Figure 43: No differences in the tissue structure of RMS with and without active KRas signaling. .87

Figure 44: oncHRas significantly increases tumor incidence and shortens latency time. . .88 


\section{List of Tables}

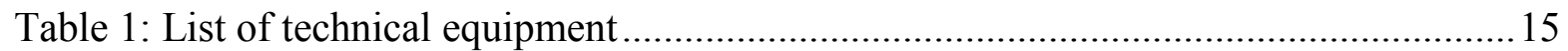

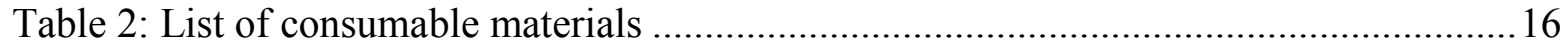

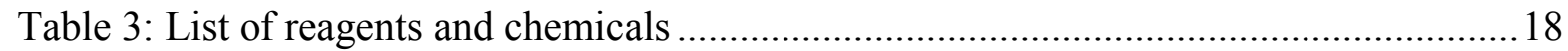

Table 4: List of kits and ready-to-use reaction systems used in this thesis.......................... 19

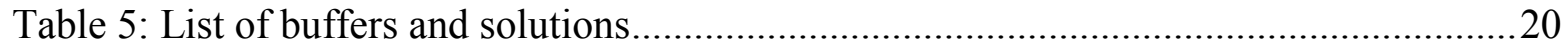

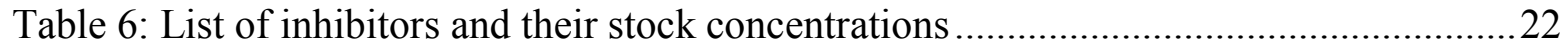

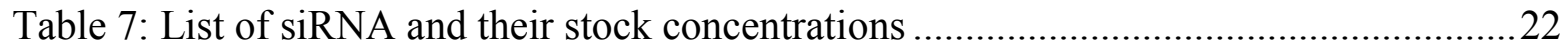

Table 8: List of supplementary materials used for the eukaryotic cells .............................23

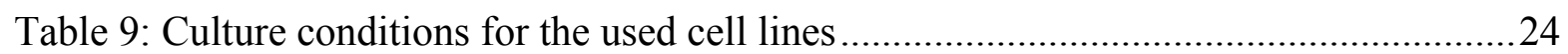

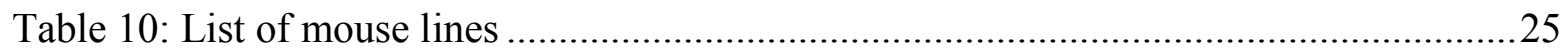

Table 11: DNA-oligonucleotides (primers) for mouse genotyping and recombination assay.

Table 12: DNA-oligonucleotides (primers) for quantitative RT PCR ................................2

Table 13: DNA-oligonucleotides (primers) for cloning and plasmid identification ..............28

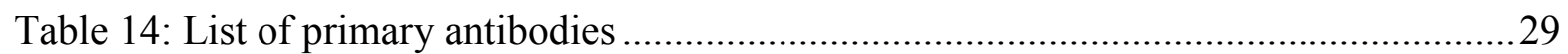

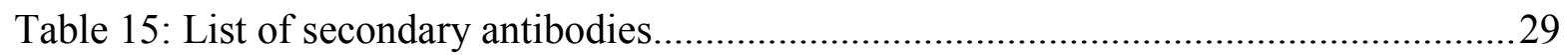

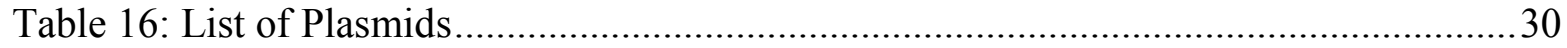

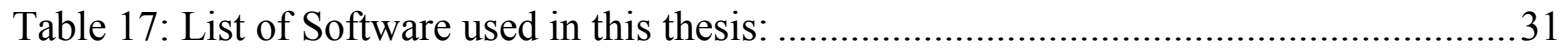

Table 18: Influence of Tamoxifen on RMS development of $\mathrm{Ptch}^{\mathrm{del} /+}$ mice .......................... 74

Table 19: Influence of oncNRas on RMS development of $\operatorname{Ptch}^{\text {del//+}} N$ RasG $12 D^{f / /}$

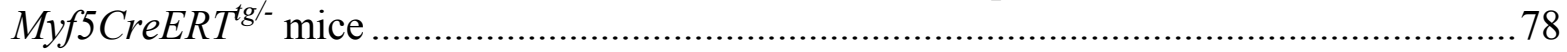

Table 20: Influence of oncKRas on RMS development of $\operatorname{Ptch}^{\text {del//+}} K R a s G 12 D^{f l /-}$

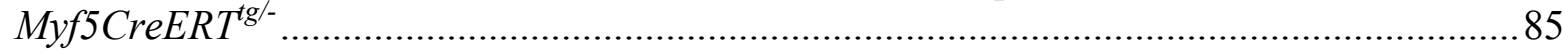

Table 21: Influence of oncHRas on RMS development of Ptch $^{\text {del//+}} H \operatorname{Ras} G 12 \mathrm{~V}^{f l /-}$ Myf5CreERT $T^{g /-}$ 



\section{Summary}

Rhabdomyosarcoma (RMS) is the most common soft tissue sarcoma in children. In children, the most common histological subtypes are embryonal RMS (ERMS) and alveolar RMS (ARMS). One feature of human RMS is aberrant Hedgehog $(\mathrm{HH})$ signaling. In mice, RMS formation occurs due to mutations in the Hh receptor Patched (Ptch), which equally activate Hh signaling. Furthermore approximately $60 \%$ of ERMS show active rat sarcoma (RAS) signaling. RAS and $\mathrm{HH}$ signaling are known to interact in several cancer types like lung, colon and pancreas. However an interdependency of these pathways in RMS is unknown.

The aim of this thesis was to analyze whether RAS and HH signaling influence each other and cooperate in the pathogenesis of RMS. For this purpose oncogenic NRAS, KRAS and HRAS (collectively named oncRAS) were overexpressed in human RMS cell lines and in Ptch mutant mice.

Indeed, oncRas signaling seems to cooperate with Hh signaling in RMS. This includes changes in the expression of Hh target genes and differences in RMS growth in Ptch mutant mice. Interestingly, the cooperation between Ras and Hh depends on the Ras isoform. Thus, the data of the genetic approach shows that expression of oncKRas and oncHRas in RMS decrease the tumor latency time and increase the tumor incidence, whereas oncNRas does not. Surprisingly, oncNRas decreases the tumor multiplicity in Ptch mutant mice and suppresses the proliferation of the tumors and thus improves the prognosis.

Furthermore, the in vitro data show that oncRAS suppresses HH signaling activity in human RMS cell lines. However the mode of action differs depending on the RMS subtype. While in the ERMS cell line RUCH-2 the RAS-mediated inhibition is caused by the MEK/ERK axis, it seems to be regulated by a jet unknown factor in the ARMS cell line RMS-13. Additionally the effects of active RAS signaling on the proliferative capacity and metabolic activity seem to vary depending on the RMS subtype. While oncRAS suppresses the proliferative capacity and metabolic activity of RUCH-2 cells, it enhances the proliferative capacity and metabolic activity of RMS-13 cells.

Together, these data indicate that HH and RAS signaling interact with each other in RMS. The outcome, however, depends on the RMS subtype and the RAS isoform. Thus, depending on the oncRas subtype the prognosis can either worsen or improve. 


\section{Introduction}

\subsection{Rhabdomyosarcoma}

Cancer in children and adolescents is rare but since 1975 the overall incidence of childhood cancer in the United states has been slowly increasing (Smith et al. 2014). Besides Neuroblastoma and Wilms-Tumor, Rhabdomyosarcoma (RMS) is the most common soft tissue sarcoma in children and adolescents. Nowadays about 350 and 80 children are diagnosed with RMS each year in the United States (American Cancer Society) and Germany (Kompetenznetz Pädiatrische Onkologie und Hämatologie), respectively. RMS belongs to the broader category of small blue round cell tumors of childhood (Wexler and LJ 1997) and histologically resembles normal fetal skeletal muscle. About $65 \%$ of the patients are younger than 6 years with a mortality rate of approximately $40 \%$ (Dagher and Helman 1999). The incidence of RMS in adults could not be clarified in total, however Sultan et al. reported that $40 \%$ of all RMS tumors are diagnosed in adults younger than 20 years (Sultan et al. 2009). In RMS patients the most common site of origin of these tumors is the head and the neck $(40 \%)$, followed by the genitourinary tract, extremities, trunk and retroperitoneum (Wang 2012). During the last decades (1975-2007), the treatment of RMS has been made great progress, which is demonstrated by an increased 5-year survival rate from 53\% to $67 \%$ in children below 15 years and from $30 \%$ to $51 \%$ in young adolescents between 15 to 19 years (Smith et al. 2014).

In children, the most common histological subtypes of RMS are alveolar (ARMS) and embryonal (ERMS) RMS. Patients with ERMS represent approximately $60 \%$ of all childhood RMS and patients with metastatic ERMS show a 5-year overall survival of $40 \%$ (Breneman et al. 2003) whereas patients with metastatic ARMS have a 5-year-overall survival of just 10$30 \%$ (De Giovanni et al. 2009). ARMS can be further divided in those with and without PAX3(7)/FOXO1 translocations resulting in respective fusion-proteins, whereas ERMS are always fusion-negative and frequently show loss of heterozygosity of $11 \mathrm{p} 15.5$ and concomitant overexpression of IGF2. Fusion-negative ARMS and ERMS in humans and in mice are characterized by pathological Hedgehog (HH)-signaling activity (Pressey et al. 2011, Zibat et al. 2010). Aberrant HH signaling activity can be monitored by the overexpression of the $\mathrm{HH}$ target gene Glioma-associated oncogene GLII. 


\subsection{Hedgehog (Hh) signaling pathway}

The Hh signaling pathway was originally identified to affect multiple processes in tissue patterning during embryonic development. Today it is known that this pathway also regulates tissue homeostasis, regeneration and tissue healing as well as stem cell activation and selfrenewal (Beachy et al. 2004, Nusslein-Volhard and Wieschaus 1980, Ruiz i Altaba et al. 2007).

Components of the Hh pathway were first described by Nusslein-Volhard and Wieschaus who performed a screen of genes involved in body patterning of Drosophila melanogaster (Nusslein-Volhard and Wieschaus 1980).

Today it is known that this pathway is highly conserved between the species, however with slight differences. Drosophila has only one Hh and Patched (Ptch) gene and one transcription factor $\mathrm{Ci}$, while vertebrates have three different $H$ h genes, Sonic hedgehog (Shh), Indian hedgehog (Ihh), and Desert hedgehog (Dhh), two Patched genes, Ptch1 and Ptch2 and 3 multiple Gli transcription factors, Gli1, Gli2 and Gli3 (Echelard et al. 1993).

Of the $3 \mathrm{Hh}$ homologs in vertebrates, Shh is the best characterized. Shh is involved in regulation of patterning of the limb bud and of the neural tube (Marigo et al. 1996, Marigo and Tabin 1996), while Ihh is specifically involved in bone and cartilage growth and differentiation and Dhh in gonadal differentiation (Bitgood et al. 1996, St-Jacques et al. 1999). The three Gli zinc-finger transcription factors in vertebrates mediate the Hh signal to the nucleus and exist as activator and repressor forms. Thus, all Gli proteins consist of a Cterminal activation domain, whereas Gli2 and Gli3 additionally have a N-terminal repressor domain (Briscoe and Therond 2013). The transcriptional activation of the pathway is mostly regulated by the full lengths Gli2/3 proteins (activator form) whereas the transcriptional repression is managed by the $\mathrm{C}$-terminally truncated Gli proteins (repressor form).

In vertebrates canonical Hh signaling is mediated by the Hh receptor Ptch, which regulates its interaction partner Smoothened (Smo) which in turn activates the Gli transcription factors. Despite the long research history on the Hh signaling pathway the interaction between the $\mathrm{Hh}$ components is quit incomplete. 
A

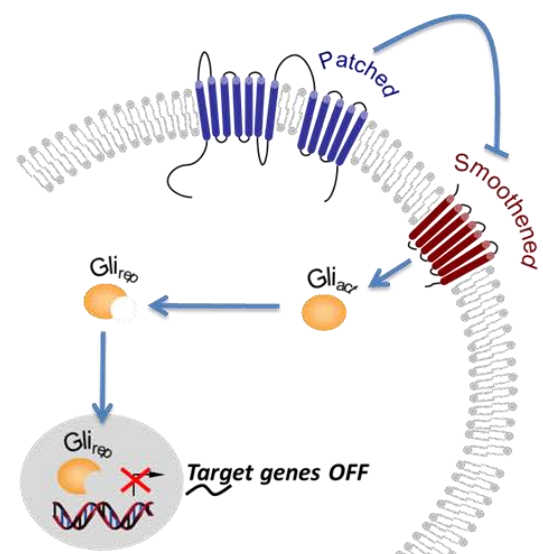

B

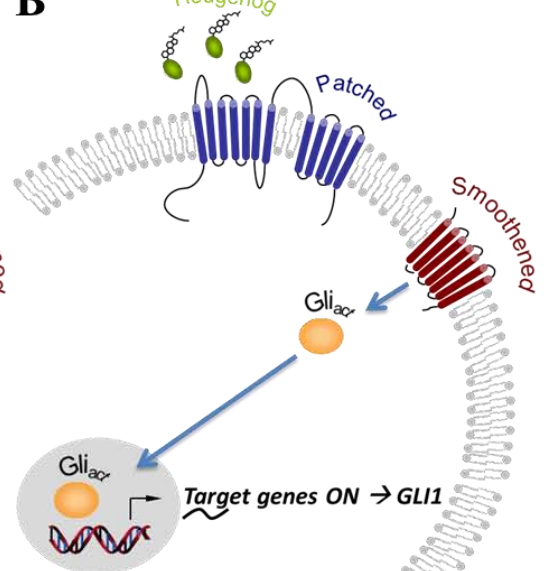

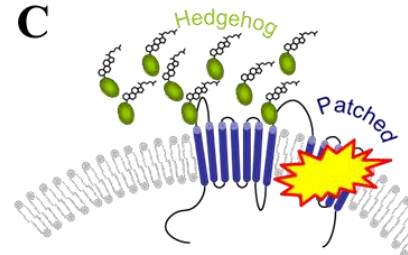

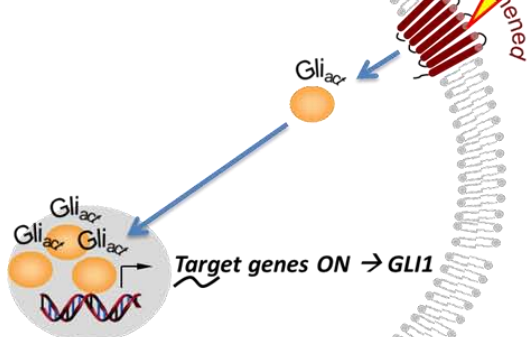

Figure 1: Simplified schematic drawing of the canonical $\mathbf{H h} / \mathrm{Ptch}$ signaling pathway. The figure shows a schematic drawing of physiological activation and pathological activation of the Hh signaling pathway. (A) In the inactive state Ptch inhibits Smo, which promotes the Gli repressor form and blocks target genes (B) In the physiological active state the ligand Hh binds to Ptch. This blocks Ptch function and results in activation of Smo, which promotes the Gli activator forms that activate target gene expression in the nucleus. (C) Ligand dependent and independent pathological activation of the Hh signaling pathway by either oversupply of the ligand $\mathrm{Hh}$ or mutation in $\mathrm{Hh}$ components (inactivating mutations in Ptch or activating mutations in Smo). The figures are modified from the Dissertations of Nitzki 2008 and Linder 2015.

In the inactive state (see Figure 1A), the ligand $\mathrm{Hh}$ is absent, resulting in the repression of the pathway by Ptch. Ptch is a 12-span transmembrane receptor that inhibits the activity of Smo. The detailed mechanism behind the inhibition of Smo by Ptch is only partially understood. In the current model Ptch prevents the translocation the seven-transmembrane G-protein-coupled receptor-like protein Smo to the primary cilium (Rohatgi et al. 2007). Blocking of accumulation of Smo at the cilium results in the degradation of the zinc finger transcription factors Gli2 and Gli3 to the transcriptional C-terminal truncated repressor forms ( $\left.\mathrm{Gli}_{\text {rep }}\right)$ (Wang et al. 2000, Wen et al. 2010). The $\mathrm{Gli}_{\text {rep }}$ forms are translocated into the nucleus and block the Hh target gene promoters and thus target gene expression (Aza-Blanc et al. 1997).

In the presence of Hh the pathway is activated (see Figure 1B) by binding of Hh to Ptch. This results in inhibition of Ptch function and in translocation of Smo, and accumulation in the primary cilium. This in turn leads to stabilization of the Gli2 and Gli3 activator forms (Gli $\mathrm{act}_{\text {at }}$ and their translocation to the nucleus where they activate transcription of target genes (Cohen 2010, Roberg-Larsen et al. 2014). One of these targets is Glil, which expression is thought to 
be a reliable marker of active Hh signaling. Another target gene is Ptch that thus is regulated in a negative feedback loop (Hooper and Scott 2005, Wetmore 2003).

When components of the Hh signaling pathway are mutated, these mutations can result in a constitutive pathological activation of the Hh pathway (Figure 1C). These mutations can be loss of function mutation of Ptch or activating mutations in Smo. In addition, pathological activation of the pathway can occur due to overexpression of Hh or Gli. Pathological activation of Hh signaling is involved in the development of various cancer types like basal cell carcinoma, several other carcinomas, medulloblastoma, multiple myeloma, chronic myeloid leukaemia (Gupta et al. 2010, Hahn et al. 1996, Liu et al. 2011) and also RMS (Zibat et al. 2010). All of these tumors overexpress Glil mRNA, which is thought to be a very good indicator for pathological Hh signaling activity and sometimes this also goes along with overexpression of Ptch mRNA (Scales and de Sauvage 2009).

Conclusively, Hh signaling pathway is an important pathway that regulates several essential functions like cell proliferation, differentiation and survival and is responsible for the proper development of numerous organs and tissues (Hooper and Scott 2005) and thus it requires an accurate onset control, strength and termination (Varjosalo and Taipale 2008).

\subsection{Hh signaling pathway and RMS}

In the past, several connections were discovered between aberrant $\mathrm{Hh}$ signaling and the pathogenesis of RMS (Bridge et al. 2000, Tostar et al. 2006, Zibat et al. 2010). First it has been shown that inherited PTCH mutations are responsible for Gorlin syndrome. Besides a predisposition to basal cell carcinoma and medulloblastoma, this syndrome predisposes the affected individual to other tumors including RMS (Hahn et al. 1996). In addition mice with inactivating Ptch or activating Smo mutations develop RMS resembling the human embryonal RMS subtype (Hahn et al. 1998, Hahn et al. 2004, Kappler et al. 2004, Mao et al. 2006) and both, human and murine RMS overexpress Hh target genes (Hahn et al. 1998, Zibat et al. 2010). Investigations on human RMS samples revealed that $33 \%$ of ERMS show loss of the PTCH locus at chromosome 9q22 (Bridge et al. 2000, Bridge et al. 2002). Furthermore HH signaling pathway is more active in ERMS and fusion negative ARMS compared to fusion positive ARMS (Zibat et al. 2010). In addition, a study of Paulson et al. showed that ERMS have a HH-signature and have high expression levels of GLI2 and GLI3 (Paulson et al. 2011). 
Finally it has been shown that the GLI inhibitor GANT-61 and the PKA activity stimulator and Shh inhibitor forskolin reduce growth and induce apoptosis by inhibiting the $\mathrm{HH}$ signaling pathway in RMS cultures and xenografts (Tostar et al. 2010, Yamanaka et al. 2011).

\subsection{Ras signaling pathway}

The rat sarcoma (Ras) signaling pathway plays a major role in normal tissue. Ras is a proto oncogene and is involved in many cellular processes like growth and differentiation. In humans, mutations in the RAS subtypes KRAS, HRAS and NRAS were found in approximately $30 \%$ of all tumors according to the current data available at the COSMIC database (http://cancer.sanger.ac.uk/cosmic) (HRAS mutations in $3 \%$, KRAS mutation in $20 \%$ and NRAS mutations in $5 \%$ of all tumors). The three human $R A S$ genes encode for $21 \mathrm{kDa}$ proteins, are highly homologous and share $85 \%$ identity at their amino acid sequence. Many mutant alleles were discovered in different human cancers and cancer cell lines for example in those of the pancreas (90\%), colon (50\%), thyroid gland (50\%) and lungs $(25 \%)$ (Der et al. 1982, Parada et al. 1982, Santos et al. 1982). Sometimes specific tumors harbor specific $R A S$ mutations. For example HRAS mutation are often identified in bladder carcinoma, whereas KRAS mutations dominate in colorectal, pancreatic, lung and cervical cancers and NRAS mutations in melanoma and liver carcinoma, lymphoid and myeloid malignancies cancer.

Ras is a guanine nucleotide-binding protein located at the inner surface of the plasma membrane. Ras activity is promoted by Guanine nucleotide Exchange Factor (GEF) or Guanine Dissociation Stimulators (GDS) and inactivated by GTPase Activating Protein (GAPs) (Boguski and McCormick 1993, Bollag and McCormick 1991). In inactive state Ras is bound to guanosine diphosphate (GDP). For activation of Ras signaling GDP has to be exchanged by guanosine triphosphate (GTP). For this purpose the GEFs or GDS form a complex with GDP bound Ras and release the GDP from the complex. The higher amount of intracellular GTP compared to GDP ensures that the released GDP is directly replaced by GTP. Next the GEFs or GDS release the Ras-GTP complex which leads to the activation of the downstream effector pathways (Bourne et al. 1990, Bourne et al. 1991). In a normal cell the activation of Ras is transient and the Ras-GTP complex is hydrolyzed by GAP to GDP, resulting in inactivation of Ras. In a cell with an activating mutation in Ras (for example in 
codons 12, 13, 59 or 61) GAP can bind to the Ras-GTP complex but is not able to hydrolyze the GTP to GDP. This results in constitutive activation of Ras and its effector pathways.

The Ras signaling pathway is a key element of different signaling pathways to transfer informations from the cell membrane to the nucleus. It is able to activate several effector pathways. The first identified and best characterized Ras effector was "rapidly accelerated fibrosarcoma" (Raf). The Ras-Raf signaling is known to regulate cell cycle progression or differentiation processes (Haigis et al. 2008). For the activation of Raf1, cytoplasmic Raf1 binds to Ras in a GTP-dependent manner and thus Raf1 is translocated to the plasma membrane. There the Rafl kinase can be activated by a Ras-independent modulation by tyrosine phosphorylation (Marais et al. 1995, Stokoe et al. 1994). Next the activated Raf1 phosphorylates its downstream target, which is the MAP kinase-extracellular signal-regulated kinase (Mek) (Cowley et al. 1994, Kyriakis et al. 1992). Phosphorylation of Mek activates and phosphorylates the extracellular-signal-regulated kinases (Erk), p44/42 Erk1 and Erk2 (Dent et al. 1992, Kyriakis et al. 1992, Robinson et al. 1996, Zheng and Guan 1993). Once activated Erk can mediate several important processes in a cell for example gene expression, cell differentiation, proliferation and programmed death (reviewed by (Mebratu and Tesfaigzi 2009)).

As mentioned above Ras can also regulate several other effector pathways. The second wellcharacterized Ras effector pathway is the antiapoptotic Phosphatidylinositol 3-kinase (PI3K) pathway. In general the PI3K pathway is involved in survival, transcription and cytoskeletal signals of a cell. The PI3K pathway is a highly complex pathway consisting of several activators, inhibitors and second messengers. In a simplified description, PI3K can be activated by binding to Ras-GTP. The active PI3K phosphorylates the membrane phospholipid phosphatidylinositol (4,5)-bisphosphate $\left(\mathrm{PIP}_{2}\right)$ to form the second messenger phosphatidylinositol (3,4,5)-trisphosphate $\left(\mathrm{PIP}_{3}\right)$ leading to recruitment of the phosphotidylinositide-dependent kinase (PDK1) and protein kinase B (known as Akt). While PDK1 is activated by recruitment to $\mathrm{PIP}_{3}$, Akt is partially phosphorylated on Thr308 by PDK1 (Martelli et al. 2010). The full activation of Akt requires another phosphorylation of the hydrophobic motif at Ser473, which can be performed by several kinases for example by mammalian target of rapamycin complex 2 (mTORC2) or DNA-dependent kinase (DNA-PK) (Bozulic and Hemmings 2009). Ones activated Akt affects several other proteins involved in 
proliferation, differentiation and cell survival (Downward 2004, Franke 2008). However, the activation of the PI3K pathway can also lead to the activation of Raf/Mek/Erk-signaling. This involves several proteins including Gab, Irs, Grb7 and Pdk1 (Aksamitiene et al. 2012). Interestingly, a bidirectional cross-talk between Erk and PI3K signaling has been described, that, dependent on the cellular context, can result in activation or inhibition of the Mek/Erk and PI3K/Akt axis, respectively (Aksamitiene et al. 2012).

Beside the regulation of apoptotic processes caused by the activation of the PI3K/Akt pathway (Downward 2003) Ras can also regulate apoptosis via Bad, Bim, Mcl-1, caspase 9 and Bcl-2 (Steelman et al. 2004). Furthermore it has been shown that beside growth promoting effects and the ability to induce tumors (Roop et al. 1986) active Ras signaling also can negatively regulate tumor growth by equalizing the growth promoting effects with protective mechanisms that decrease proliferation (Serrano et al. 1997). Indeed, several research groups showed that Ras is able to induced senescence in vivo in mouse models as well as in human cell lines (reviewed in Courtois-Cox, Jones et al. 2008). For example Courtois-Cox et al. reported that activation of RAS signaling in benign human tumors resulted in activation of the two tumor suppressors RB and TP53 and senescence (CourtoisCox et al. 2008). In this context several factors and complexes were regulated including NF$\kappa \mathrm{B}$ (Downward 1998), cAMP signaling (Shirokawa et al. 2000), and the Nore1-RASSF1Mst1 complex (Feig and Buchsbaum 2002).

\subsection{Ras signaling pathway and RMS}

RAS mutations were also detected in human RMS. Thus, patients with Costello syndrome, which is caused by germline mutations in HRAS, are predisposed to ERMS. Moreover, up to $42 \%$ of ERMS show activating mutation in either KRAS, HRAS or NRAS with mutations in NRAS being the most common ones (Chen et al. 2013, Martinelli et al. 2009, Paulson et al. 2011, Shern et al. 2014, Stratton et al. 1989). Furthermore, alternative mutations can result in activation of RAS signaling for exsampe loss of function mutations in Neurofibromin 1 (NF1) (Paulson et al. 2011). Since NF1 normally regulates GAP to promote the hydrolysis of GTP to GDP, loss of NF1 function results in activation of RAS. Together, approximately $60 \%$ of all ERMS exhibit active RAS signaling. Interestingly, just one case with an activating RAS mutation has been described in the alveolar subtype of RMS (Shern et al. 2014). 
It has been shown that induction of KRas mutations in mice with a preexisting mutation in p53 results in the development of pleomorphic RMS (Doyle et al. 2010, Tsumura et al. 2006). Moreover, Langenau et al. expressed an oncogenic KRas mutation under the rag2 promotor in a zebrafish that resulted in ERMS-like tumors with a tumor-specific gene expression profile comparable to human ERMS (Langenau et al. 2007). These data suggest that Ras signaling may play a hitherto underestimated role in RMS, especially in the embryonal subtype. However, the exact role of oncogenic RAS in RMS is unknown. Recently, Rubin et al. published microarray-based results showing that a RAS signature in ERMS only occurs in connection with signatures of other active signaling pathways (e.g. HH signaling on, RB or TP53 Signaling-off) (Rubin et al. 2011). Therefore the authors assume that RAS Signaling probably plays a role as a modifier with respect to ERMS initiation.

\subsection{Interaction between $\mathrm{Hh}$ and Ras signaling}

Beside the canonical regulation of Hh signaling i.e. via the $\mathrm{Hh} / \mathrm{Ptch} / \mathrm{Smo} / \mathrm{Gli}$ axis, the activity of Gli transcription factors can be modulated by other molecules or signaling pathway. These Gli modulators can act independently of the Hh ligand and in a Smo independent noncanonical manner. The Smo independent non-canonical regulation of Gli activity involves several molecules and signaling pathways that can bypass the ligand-dependent $\mathrm{Hh}$ signaling axis and include Ras signaling that via the Mek/Erk axis can stimulate Gli activity. Ras also can stimulate Gli activity via the PI3K and Pkc axis (Figure 2). 


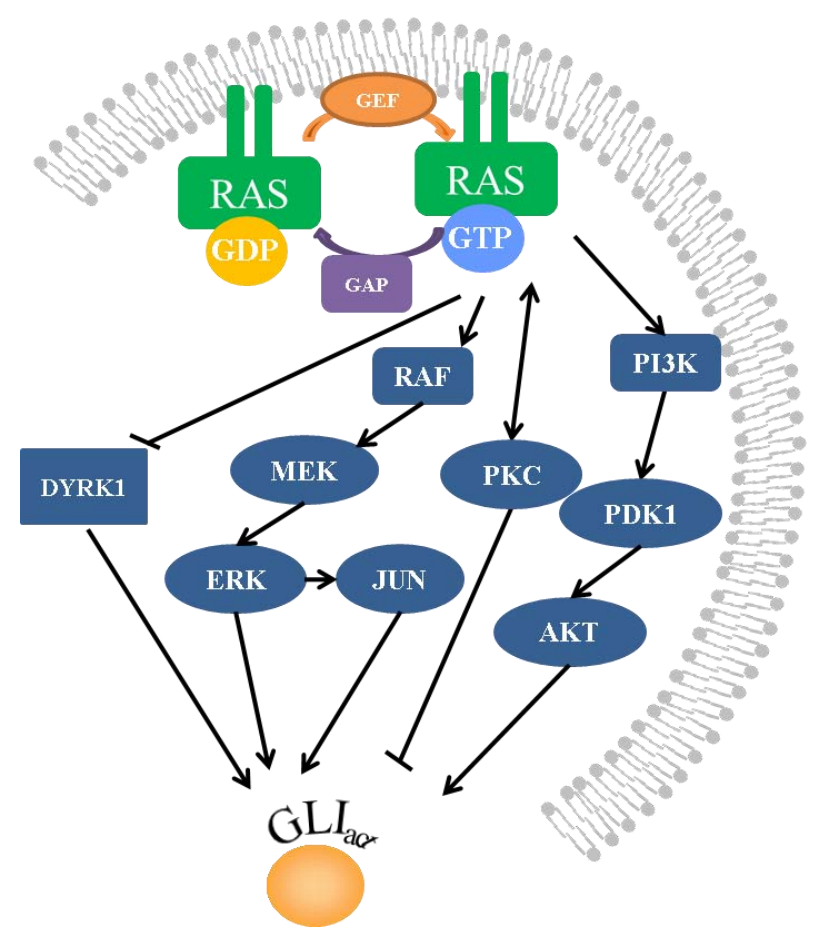

Figure 2: Schematic drawing possible interactions between RAS and GLI activation. The figure shows a schematic drawing of the possible regulators of Smo independent non-canonical activation of GLI by active RAS signaling. Modified from Aberger (Aberger et al. 2012).

In the literature, the data regarding the interaction of $\mathrm{HH}$ - and RAS-Signaling varies depending on the tumor entity. These tumors include those of the pancreas, lung and colon. This coincidence indicates a possible cooperation between the two signaling pathways involved in tumorigenesis and / or tumor maintenance. Indeed, it was shown that RAF/MEK/ERK signaling can have a positive regulatory role in GLI transcriptional activity in gastric cancer cells (Seto et al. 2009). Upon that it was reported that this involves modulation of GLI phosphorylation (Niewiadomski et al. 2014). This indicates that the MEK/ERK axis can directly regulate the activity of GLI. Furthermore it has been reported that the MEK/ERK axis prevents the degradation of GLI protein (Ji et al. 2007). Indeed, in keratinocytes activation of ERK1/2 by EGFR signaling results in stabilization of GLI proteins. This was shown for GLI2 that was stabilized by EGFR-mediated inhibition of the proteasome pathway (Kasper et al. 2006). In addition, PI3K/Akt signaling that is also downstream of Ras, can influence Gli activity. Thus, Riobo and colleagues showed that the PI3K/Akt pathway increases Shh induced Gli activation (Riobo et al. 2006b).

Moreover, as published by Stecca et al. for melanoma, activating RAS mutations can also result in activation of the HH-signaling cascade via activation of GLI1 (Stecca et al. 2007). 
The authors showed that activation of RAS signaling either through the RAF/MEK/ERK or $\mathrm{PI} 3 \mathrm{~K} / \mathrm{AKT}$ axis can enhance the nuclear localization and transcriptional activity of GLI. In addition, Riobo et al. showed that GLI can also be activated through the PKC $/ \mathrm{MEK} / \mathrm{ERK}$ axis, which also belongs to the RAS effector pathways (Riobo et al. 2006a).

In contrast, as recently shown by Lauth et al. for pancreas carcinoma, active RAS signaling suppresses the activity of the transcription factors GLI2 and GLI3 by modulating the protein kinase DYRK1B. The latter mechanisms result in a tumor intrinsic inhibition of the HHsignaling cascade (Lauth et al. 2010), although the tumors concomitantly express and secrete SHH.

Taken together these data show that Ras signaling can either activate or inhibit Hh signaling, which strongly depends on the cellular context.

\subsection{Interaction between Hh and Ras signaling in RMS}

There have been several studies on the role of Hh signaling in RMS as well as on Ras signaling in RMS. However, it is completely unknown whether both pathways interact with each other in this tumor entity. Nevertheless, there is data strongly supporting connectivity between both pathways. Thus, as already mentioned above, a connection between those pathways could be investigated in several other tumor entities and due to the fact that approximately $60 \%$ of ERMS tumors show active RAS signaling, which is caused by either loss of function of NF1 (15\%) or activating mutation in RAS (42\%) itself (Paulson et al. 2011) and HH signaling is mainly active in fusion negative ARMS and ERMS (Pressey et al. 2011, Zibat et al. 2010).

\subsection{Mouse models used in this study}

In the present work five mouse strains were used to investigate the role of oncogenic RAS signaling in Hh-associated RMS. These were conventional Ptch deficient mice $\left(\right.$ Ptch $\left.^{\text {del/ }}\right)$, a tamoxifen inducible, muscle-specific Cre-driver $\left(M y f 5 C r e E R T^{t g /-}\right)$ and three strains that conditionally can express oncogenic N-, K- or HRas (NRasG12D $D^{f l /}, K R a s G 12 D^{f l /-}$ and $\left.\operatorname{HRas} G 12 V^{f l /-}\right)$. 


\subsubsection{Ptch deficient mice: A mouse model to study the pathogenesis of ERMS}

In the presented work Ptch $^{\text {del/+ }}$ mice were used. This mouse model was generated by breeding conditional knockout Ptch ${ }^{\text {floxfllox }}$ to EIIaCre mice (Lakso et al. 1996). In Ptch ${ }^{\text {floxflox }}$ mice exon 8 and 9 of the Ptch locus are flanked by loxP sites (Uhmann et al. 2007). The EIIaCre mouse expresses a Cre recombinase under control of the endogenous EIIa promoter. The expression is most efficient in mature oocytes. To achieve a germline deletion, Ptch floxflox mice were crossed with EIIaCre mice (for exact crossing see (Zibat et al. 2009)). In the resulting heterozygous $\mathrm{Ptch}^{\mathrm{del} / \mathrm{+}}$ mice exons 8 and 9 of the Ptch gene are excised. This leads to the expression of an aberrant Ptch transcript with exon 7 spliced into exon 10 resulting in a frameshift and a premature stop codon. Ptch ${ }^{\text {del/+ }}$ mice were backcrossed 10 times to a pure Balb/cJ background that confers high susceptibility to RMS resembling the human embryonal RMS subtype (Hahn et al. 2004).

\subsubsection{Conditional Ras Mice: Tools for the modulation of oncogenic Ras signaling in specific cells}

Three mouse strains were used in this study that allowed conditional expression of human variants of oncogenic Ras. These were the strains $L S L-N R a s^{G 12 D}$ (Haigis et al. 2008), LSLKRas $^{G 12 D}$ (Tuveson et al. 2004) and FR-HRas ${ }^{G 12 V}$ (Chen et al. 2009).

For the generation of the LSL-NRas ${ }^{G 12 D}$ strain Haigis and colleagues used V26.2 murine embryonic stem (ES) cells derived from the C57BL/6 strain to insert a floxed STOP element (LSL) followed by the first two exons of oncogenic $N R a s^{G 12 D}$ into the wildtype locus of NRas. Due to the STOP element this constitutes a null NRas allele. Upon expression of Cre, the STOP element is excised and the activated allele of NRas (NRasG12D) is expressed. Because the mutant allele is expressed from its endogenous promoter, murine NRasG12D is expressed at the same level and in the same pattern as wild-type (wt) NRas (Haigis et al. 2008). The mice are born according to Mendelian ratio, are viable and fertile and can be kept homo- or heterozygote.

The Cre-dependent conditional LSL-KRas ${ }^{G 12 D}$ mouse was generated by Tuveson et al. who designed a targeting vector containing the G12D point mutation in exon 2 of the KRas allele and a floxed STOP element upstream of the mutation. Thus, similarly to the LSL-NRas G12D strain, expression of oncogenic KRas is controlled by a removable transcriptional termination 
STOP element. This targeting vector was electroporated into 129S4/SvJae-derived J1 embryonic stem cells and the mice carrying the $L S L-K R a s^{G 12 D}$ allele were backcrossed to C57BL/6 (Tuveson et al. 2004). In the presence of a Cre recombinase the STOP element is removed and the oncogenic allele is expressed at the same level and pattern as the $w t$ allele. The mice are born according to Mendelian ratio, are viable and fertile and can be kept heterozygote.

For the development of the Cre-dependent conditional FR-HRas ${ }^{G 12 V}$ mouse Chen and coworkers transfected the targeting vector into 129SvEv (129S6) ES cell lines. The vector harbored a floxed HRas wt sequence followed by a Neo cassette flanked by Frt sites and a HRas $^{G 12 V}$ sequence. The FR-Hras ${ }^{G 12 V}$-Neo mice were crossed with the $\beta$-actin-Flp mice to remove the Neo cassette. The resulting offspring were used for further breedings and experiments (Chen et al. 2009). In the presence of a Cre recombinase the wt HRas sequence is excised and the HRas ${ }^{G 12 V}$ sequence is expressed. The mice were born according to Mendelian ratio, are viable, fertile, and survived normally. It was reported by Chen et al that they can have systemic hypertension and when aged they can develop myocardial and kidney fibrosis.

\subsubsection{Myf5creERT mice: Tool for induction of conditional mutations in $M y f 5$ expressing cells}

The Myf5 gene (Myogenic factor 5) belongs to the muscle specific determination genes and is important for skeletal muscle development. In order to generate a mouse line that allows targeting of cells expressing Myf5 at different developmental stages Biressi et al. generated a tamoxifen inducible Myf5creERT mouse line. For this purpose they designed a target vector that placed a tamoxifen-inducible Cre recombinase (ires-CreER ${ }^{\text {TM}}$-FRT-Neo-FRT cassette) in the 3' untranslated region of the Myf5 gene behind the Myf5 STOP codon (Biressi et al. 2013). Thus, the Cre recombinase is expressed from the Myf5 locus. The mice are born at a Mendelian ratio, are viable and fertile and can be kept homo- or heterozygote.

Since ERMS and fusion negative ARMS in humans and RMS of Ptch mutant mice show very high Myf5 expression in comparison to normal skeletal muscle (Nitzki et al. 2011, Zibat et al. 2010) Myf5creERT mice were considered to be useful for RMS-specific expression of the above mentioned oncogenic Ras genes. 


\section{Aim of the studies}

The primary aim of this study was to investigate the interaction of $\mathrm{Hh}$ and Ras signaling in RMS. This involved also the investigation of the role of oncogenic Ras variants in development and pathogenesis of RMS. Probably, this study will offer new therapy option for the treatment of RMS. For this purpose, in vitro and in vivo studies were performed.

First, 2 human RMS cell lines, which were wildtype for RAS (RUCH-2 (ERMS) and RMS-13 (ARMS)), were stably transduced with viral vectors expressing either oncogenic NRASG12V, KRASG12V or HRASG12V. After transduction, the RMS cell lines were examined with respect to $\mathrm{HH}$ signaling activity, cell viability and also to proliferative behavior both in culture and in nude mice.

Second, the influence of all 3 oncogenic Ras genes on the growth behavior of Hh-associated ERMS was studied in a genetic approach. For this purpose Ptch deficient mice were crossed with mice that conditionally express oncogenic N-, K- or HRas. To drive the expression of oncogenic Ras specifically in RMS, the mice were crossed to Myf5CreERT mice. In the resulting offspring the expression of oncogenic Ras was activated shortly after birth by tamoxifen injection. If possible, the mice were monitored for up to 200 days and the influence of oncogenic Ras on tumor incidence, latency time and multiplicity was investigated by comparison with control mice. Furthermore the tumors were subjected to histological and molecular analyses.

Excursion: In a first experiment the tamoxifen-inducible HSACreERT2 ${ }^{\text {tg/- }}$ Cre-driver was used that expresses the Cre recombinase specifically in skeletal muscle under the control of the human skeletal muscle actin promoter (Schuler et al. 2005). These mice were crossed to Ptch $^{\text {del/+ }}$ oncRas ${ }^{f l /-}$ mice. In the resulting Ptch ${ }^{\text {del/ }}$ oncRas $^{f l /-} H S A C r e E R T 2^{\text {tg/- }}$ mice the expression of mutant NRas, KRas or HRas was induced at an age of 4 weeks by tamoxifen. The mice were monitored weekly for up to an age of 200 days. However, very unfortunately, at the end of October 2013 it was discovered that the HSACreER ${ }^{T 2}$ is active in the skeletal muscle of the induced mice but not in RMS of these animals. Therefore we switched to the Myf5creERT ${ }^{\text {tg/- }}$ mouse model. 


\section{Materials and Methods}

\subsection{Technical equipment}

The technical equipments used in this thesis are listed in Table 1.

Table 1: List of technical equipment

\begin{tabular}{|c|c|}
\hline Equipment & Supplier \\
\hline$-80^{\circ}$ C Freezer(MDF-U71V) & Sanyo Electric Co., Ltd., Japan \\
\hline 8-well pipette & Costar, Corning Incorporated, Corning, USA \\
\hline Agarose gel electrophoresis chamber & Peqlab Biotechnologie GmbH, Erlangen \\
\hline Arium ${ }^{\circledR} 611$ VF water purification system & Sartorius, Göttingen \\
\hline Autoclave (sanoclav) & W. Krannich GmbH \& Co. KG, Göttingen \\
\hline Gas burner (Gasprofi 2 scs) & WLD-TEC GmbH, Göttingen \\
\hline $\begin{array}{l}\text { Centrifuges (Biofuge pico, fresco, primo, } \\
\text { Multifuge 3LR) }\end{array}$ & $\begin{array}{l}\text { Kendro Laboratory Products GmbH, Hanau, } \\
\text { German }\end{array}$ \\
\hline Clean bench (Euroflow Class IIA) & Clean Air Techniek bv, Woerden, Netherlands \\
\hline Cryostat (Modell CM 1900-1-1) & Leica Microsysteme Vertrieb GmbH, Bensheim \\
\hline Cell counter & Omnilab-Krannich, Göttingen, Germany \\
\hline Digital Monochrome Printer P91D & Mitsubishi, Ratingen \\
\hline Digital Photo camera (PowerShot G2) & Canon Inc., Japan \\
\hline FACScalibur & BD Biosciences GmbH, Heidelberg \\
\hline Fluorchem Q & Fisher Scientific GmbH, Schwerte \\
\hline Freezer $\left(-20^{\circ} \mathrm{C}\right)$ & Liebherr GmbH, Ochshausen \\
\hline Fridge $\left(4^{\circ} \mathrm{C}\right)$ & Robert Bosch GmbH, Stuttgart \\
\hline Heating block (Thermomixer) & Eppendorf AG, Hamburg \\
\hline Heating and stirring plate (MR 3000/3001) & Heidolph Instruments, Schwabach \\
\hline Homogenizer (Miccra D-1) & ART Prozess- \& Labortechnik GmbH \& Co. \\
\hline Hybridization oven (HB-1000 Hybridizer) & UVP, Inc., Upland, USA \\
\hline Incubators $\mathrm{CO} 2(6000, \mathrm{BBD} 6220)$ & Kendro Laboratory Products GmbH, Hanau \\
\hline $\begin{array}{l}\text { Inverse microscope with fluorescence filter } \\
\text { (Axiovert 25,Filter Set } 43,01,09 \text { ) }\end{array}$ & Carl Zeiss Jena GmbH, Jena \\
\hline Liquid nitrogen tank & L'air liquide S.A., Paris \\
\hline Luminometer (Synergy Mx) & BioTek Instruments, Inc., Bad Friedrichshall \\
\hline Mastercycler ep gradient $S$ & Eppendorf AG, Hamburg \\
\hline Microtome (HN 40) & Leica Microsysteme Vertrieb $\mathrm{GmbH}$, Bensheim, \\
\hline
\end{tabular}




\begin{tabular}{|c|c|}
\hline & Germany \\
\hline MilliQ-water purifier & MembraPure GmbH, Bodenheim \\
\hline Mini centrifuge & Carl Roth GmbH, Karlsruhe \\
\hline Mr. FrostyTM Freezing Container & Thermo Fisher Scientific GmbH, Schwerte \\
\hline Multipette & Eppendorf AG, Hamburg \\
\hline Nanodrop & Thermo Fisher Scientific GmbH, Schwerte \\
\hline $\begin{array}{l}\text { Paraffin dispenser, embedding machine } \\
\text { (Dispenser PAG12) }\end{array}$ & Medite Medizintechnik GmbH, Burgdorf \\
\hline PCR-machine & Eppendorf, Hamburg \\
\hline PCR-machine & BIORON Diagnostics GmbH, Ludwigshafen \\
\hline pH-meter (inoLab pH Level 1) & WTW GmbH, Vienna, Austria \\
\hline Pipette controller(Accu-jet) & Brand GmbH \& Co. KG, Wertheim, Germany \\
\hline Power source for electrophoresis & $\begin{array}{l}\text { Peqlab Biotechnologie } \mathrm{GmbH}, \text { Erlangen, } \\
\text { Germany }\end{array}$ \\
\hline Sequencer (ABI 3500 XL) & Life Technologies GmbH, Darmstadt \\
\hline Shaking incubator & New Brunswick Scientific GmbH, Nürtingen \\
\hline Single channel pipettes & Eppendorf AG, Hamburg \\
\hline Sterile bench (Euroflow class IIA) & Clean Air Techniek bv, Woerden, Netherlands \\
\hline TaqMan (ABI Prism 7900HT) & Life Technologies GmbH, Darmstadt \\
\hline $\begin{array}{l}\text { UV-printer (Digital Monochrome Printer } \\
\text { P91D) }\end{array}$ & Mitsubishi, Ratingen, Germany \\
\hline Trans-Blot SD semi-dry transfer cell & Bio-Rad Laboratories GmbH, Munich \\
\hline Vortexer (Vortex-Genie 2) & Scientific Industries, Inc., Bohemia, USA \\
\hline Weighing scale (Sartorius Basic plus) & Sartorius AG, Göttingen \\
\hline
\end{tabular}

\subsection{Consumable materials}

The consumable materials used in this thesis are listed in Table 2.

Table 2: List of consumable materials

\begin{tabular}{ll}
\hline Consumable materials & Supplier \\
\hline $\mathbf{1 . 5} \mathbf{~ m l}$ reaction tubes & Ochs GmbH, Bovenden/Lenglern \\
$\mathbf{1 . 5} \mathbf{~ m l ~ S a f e s e a l ~ M i c r o t u b e s ~}$ & Sarstedt AG \& Co., Nürnberg \\
$\mathbf{1 5} \mathbf{~ m l ~ t u b e s}$ & Greiner Bio-One GmbH, Frickenhausen \\
$\mathbf{2 . 0} \mathbf{~ m l ~ r e a c t i o n ~ t u b e s ~}$ & Sarstedt AG \& Co., Nürnberg \\
\hline
\end{tabular}




\begin{tabular}{|c|c|}
\hline 384-well Optical Reaction Plate & Life Technologies GmbH, Darmstadt \\
\hline $50 \mathrm{ml}$ tubes & Greiner Bio-One GmbH, Frickenhausen \\
\hline 96-Well Assay Plate & Costar, Corning Incorporated, Corning, USA \\
\hline 96-Well Optical Reaction Plate & Life Technologies GmbH, Darmstadt \\
\hline Blotting paper (GB 33 B003) & Heinemann Labortechnik GmbH, Duderstadt \\
\hline Cell culture dishes, $100 \mathrm{~mm}$ (Nunclon Surface) & Nunc GmbH \& Co.KG, Wiesbaden \\
\hline Cell scraper & Sarstedt AG \& Co., Nürnberg \\
\hline Coverslips & Menzel GmbH \& Co.KG, Braunschweig \\
\hline Cryo Pure & Sarstedt AG \& Co., Nürnberg \\
\hline Disposable needles (Sterican Ø 0,45 x 12 mm) & B. Braun Medical AG, Emmenbrücke, Germany \\
\hline $\begin{array}{l}\text { Eppendorf Combitips Plus/Advanced }(0.2,0.5 \text {, } \\
2.5,5,10,25,50 \mathrm{ml})\end{array}$ & Eppendorf AG, Hamburg \\
\hline Filter tips $(10 \mu \mathrm{l})$ & Sarstedt AG \& Co., Nürnberg \\
\hline Filter tips $(100 \mu \mathrm{l}, 200 \mu \mathrm{l}, 1000 \mu \mathrm{l})$ & Kisker Biotech GmbH \& Co. KG, Steinfurt \\
\hline Flow Cytometry Tube & Sarstedt AG \& Co., Nürnberg \\
\hline Fluted filters & Sartorius AG, Göttingen \\
\hline Glassware & Schott AG, Mainz \\
\hline $\begin{array}{lll}\text { Milliporefilter } & \text { (Nuclepore } & \text { Track-Etch } \\
\text { Membran) } & & \end{array}$ & Whatman GmbH, Dassel \\
\hline Miscroscope slides (SuperFrost Plus) & Menzel GmbH \& Co.KG, Braunschweig \\
\hline Neubauer counting chamber & Brand GmbH \& Co KG, Wertheim \\
\hline Nitrocellulose membrane(Hybond ECL) & GE Healthcare Europe GmbH, Freiburg \\
\hline NuPAGE Novex 4 - $12 \%$ Bis-Tris Midi Gel & Invitrogen $\mathrm{GmbH}$, Karlsruhe \\
\hline Pasteur pipettes & Brand GmbH \& Co.KG, Wertheim \\
\hline $\begin{array}{l}\text { PCR-Reaction tubes (ThermoFast 96, } \\
\text { nonskirted, natural domed cap strips) }\end{array}$ & Sarstedt AG \& Co., Nürnberg \\
\hline Petri dishes & Ochs GmbH, Bovenden/Lenglern \\
\hline Pipette tips $(10 \mu \mathrm{l}, 200 \mu \mathrm{l})$ & Ochs GmbH, Bovenden/Lenglern \\
\hline Pipette tips $(1000 \mu \mathrm{l})$ & Sarstedt AG \& Co., Nürnberg \\
\hline Scalpel blade & Aesculap AG \& Co.KG, Tuttlingen \\
\hline $\begin{array}{l}\text { Serological pipettes }(2 \mathrm{ml}, 5 \mathrm{ml}, 10 \mathrm{ml}, 25 \mathrm{ml} \text {, } \\
50 \mathrm{ml})\end{array}$ & Sarstedt AG \& Co., Nürnberg \\
\hline Tissue Culture Plate 6-Well & Sarstedt AG \& Co., Nürnberg \\
\hline Urine cup & Sarstedt AG \& Co., Nürnberg \\
\hline
\end{tabular}




\subsection{Reagents and Chemicals}

All chemicals which are not listed in Table 3 were purchased from AppliChem GmbH, Darmstadt, Carl Roth GmbH \& Co. KG, Karlsruhe, or from Sigma-Aldrich Chemistry GmbH, Steinheim.

Table 3: List of reagents and chemicals

\begin{tabular}{|c|c|}
\hline Reagents and Chemicals & Supplier \\
\hline 1 kb DNA, 50 bp und 100 bp DNA Ladder & Invitrogen $\mathrm{GmbH}$, Karlsruhe, Germany \\
\hline $99,9 \%$ Ethanol & J.T. Baker B.V., Deventer, Netherlands \\
\hline 99,9 \% Methylated Ethanol & $\begin{array}{l}\text { CVH Chemie-Vertrieb GmbH \& Co. Hannover } \\
\text { KG, Hannover }\end{array}$ \\
\hline Agarose & Invitrogen GmbH, Karlsruhe, Germany \\
\hline Ampuwa & $\begin{array}{l}\text { Fresenius Kabi Deutschland GmbH, Bad } \\
\text { Homburg, }\end{array}$ \\
\hline Boric acid & MP Biomedicals LLC, Illkirch, France \\
\hline Cryoblock embedding medium & $\begin{array}{l}\text { Medite Medizintechnik GmbH, Burgdorf, } \\
\text { Germany }\end{array}$ \\
\hline Deoxyribonucleotidtriphosphate (dNTP) & Roche Diagnostics GmbH, Mannheim, Germany \\
\hline Dithiotreitol, 100mM (DTT) & Invitrogen $\mathrm{GmbH}$, Karlsruhe \\
\hline Ethidium bromide (0.07 \%) & inna-TRAIN-Diagnostics, Kronberg \\
\hline Ethylenediaminetetraacetic acid (EDTA) & ICN Biochemicals Inc., Aurora, USA \\
\hline Formamide & Acros Organics b.v.b.a, Geel, Belgium \\
\hline Glycer gel mounting medium & Dako GmbH, Hamburg, Germany \\
\hline Immuno Mount & $\begin{array}{l}\text { Shandon Thermo Electron Corporation, } \\
\text { Waltham, USA }\end{array}$ \\
\hline Ketanest S (25 mg/ml) & Pfizer Pharma GmbH, Karlsruhe, Germany \\
\hline Mayer's Hemalaun solution & Merck KgaA, Darmstadt, Germany \\
\hline $\begin{array}{l}\text { Mounting medium (Vectrashield Mounting } \\
\text { Medium with DAPI) }\end{array}$ & Vector Laboratories, Inc., Burlingame, USA \\
\hline NuPAGE MES SDS Running Buffer, $20 \mathrm{x}$ & Invitrogen $\mathrm{GmbH}$, Karlsruhe, Germany \\
\hline Oligo(dT)-Nucleotide (50 ng/ $\mu \mathrm{l})$ & Roche Diagnostics GmbH, Mannheim, Germany \\
\hline Paraformaldehyde & $\begin{array}{l}\text { Carl Roth GmbH \& Co. KG, Karlsruhe, } \\
\text { Germany }\end{array}$ \\
\hline PBS tablets; ready to use solution & GIBCO Invitrogen GmbH, Karlsruhe, Germany \\
\hline Penicillin $\quad(10000 \quad \mathrm{U} / \mathrm{ml}) /$ Streptomycin $\quad(10$ & PAN Biotech GmbH, Aidenbach, Germany \\
\hline
\end{tabular}


$\mathrm{mg} / \mathrm{ml})$

RNAse-DNAse free water

SeeBlue ${ }^{\circledR}$ Plus2 Pre-Stained Standard

Sodiumdodecylsulfate (SDS)

SOC Medium

Tween-20

water (Ampuwa)

WST-1 reagent

X-Gal
Invitrogen $\mathrm{GmbH}$, Karlsruhe, Germany

Invitrogen $\mathrm{GmbH}$, Karlsruhe, Germany

Carl Roth GmbH \& Co. KG, Karlsruhe, Germany

Invitrogen $\mathrm{GmbH}$, Karlsruhe, Germany

Scharlau Chemie S.A., Barcelona, Spain

Fresenius Kabi Deutschland $\mathrm{GmbH}, \mathrm{Bad}$

Homburg, Germany

Roche Diagnostics GmbH, Mannheim, Germany

Carl Roth GmbH \& Co. KG, Karlsruhe,

Germany

\subsection{Kits and ready-to-use reaction systems}

If not stated otherwise all kits and ready-to-use reaction systems mentioned in Table 4 were used as described in the manufacturer's instructions.

Table 4: List of kits and ready-to-use reaction systems used in this thesis

\begin{tabular}{|c|c|}
\hline Kits and ready-to-use reaction systems & Supplier \\
\hline $\begin{array}{l}\text { Amersham ECL Plus }{ }^{\text {TM }} \text { Western Blotting } \\
\text { Detection Reagents }\end{array}$ & $\begin{array}{l}\text { GE Healthcare Europe GmbH, Freiburg, } \\
\text { Germany }\end{array}$ \\
\hline $\begin{array}{l}\text { Cell Proliferation } \\
\text { (chemiluminescence) }\end{array}$ & Roche Diagnostics GmbH, Mannheim, Germany \\
\hline Cell Proliferation Reagent WST-1 & Roche Diagnostics GmbH, Mannheim, Germany \\
\hline MolTaq Taq- Polymerase & Molzym GmbH \& Co. KG, Bremen \\
\hline Pierce $\circledR^{\circledR}$ BCA Protein Assay Kit & Thermo Fisher Scientific, Rockford, USA \\
\hline PureLink HiPure Plasmid Midiprep Kit & Invitrogen $\mathrm{GmbH}$, Karlsruhe, Germany \\
\hline PureLink HiPure Plasmid Miniprep Kit & Invitrogen $\mathrm{GmbH}$, Karlsruhe, Germany \\
\hline QuantiTect SYBR Green PCR & Qiagen GmbH, Hilden, Germany \\
\hline Ras Activation ELISA ASSAY Kit & Millipoor S.A.S, Molsheim Cedex, France \\
\hline SuperScriptII ${ }^{\circledR}$ Reverse Transcriptase & Invitrogen $\mathrm{GmbH}$, Karlsruhe, Germany \\
\hline SYBR Green Invitrogen & Invitrogen $\mathrm{GmbH}$, Karlsruhe, Germany \\
\hline TRIzol® Reagent & Invitrogen $\mathrm{GmbH}$, Karlsruhe, Germany \\
\hline QIAquick Gel Extraction Kit & Qiagen GmbH, Hilden, Germany \\
\hline
\end{tabular}




\subsection{Buffers and solutions}

All buffers and solutions mentioned in Table 5 were prepared in double distilled water (Satorius AG, Göttingen).

Table 5: List of buffers and solutions

\begin{tabular}{|c|c|c|}
\hline Buffer or solution & Compositions & \\
\hline 6 x SDS loading buffer & $35 \%(\mathrm{v} / \mathrm{v})$ & Glycerol \\
\hline & $9 \%(w / v)$ & SDS \\
\hline & $8.5 \%(\mathrm{w} / \mathrm{v})$ & DTT \\
\hline & $0.1 \%(\mathrm{w} / \mathrm{v})$ & Bromphenolblue \\
\hline & & in upper gel buffer \\
\hline AEC chromogen, pH 5 & $230 \mathrm{mM}$ & Acetic acid \\
\hline & $70 \mathrm{mM}$ & Sodium acetate trihydrate \\
\hline & $16 \mathrm{mM}$ & 3-Amino-9 Ethylcarbazole (dissolved \\
\hline & & in dimethyl formamide) \\
\hline Blotting buffer & $6 \%(\mathrm{w} / \mathrm{v})$ & Tris \\
\hline & $3 \%(w / v)$ & Glycine \\
\hline & $0.075 \%(\mathrm{w} / \mathrm{v})$ & SDS \\
\hline & $20 \%(\mathrm{v} / \mathrm{v})$ & Methanol \\
\hline BSA/Azidee-TBST-Solution & $0,02 \%(\mathrm{w} / \mathrm{v})$ & Sodium Azidee solution \\
\hline & $2 \%(\mathrm{w} / \mathrm{v})$ & BSA \\
\hline & & $1 \mathrm{x}$ TBST \\
\hline Citric Acid buffer, pH6 & $10 \mathrm{mM}$ & Sodium citrate $\mathrm{pH} 6.0$ \\
\hline Cresol & $0,1 \%(\mathrm{w} / \mathrm{v})$ & Cresol \\
\hline & & Saturated sucrose solution \\
\hline Eosin, $1 \%$ & $1 \%(\mathrm{w} / \mathrm{v})$ & Eosin y (water soluble) \\
\hline & $80 \%(\mathrm{v} / \mathrm{v})$ & Ethanol \\
\hline LacZ-staining buffer & $5 \mathrm{mM}$ & $\mathrm{K} 3 \mathrm{Fe}(\mathrm{CN}) 6$ \\
\hline & $5 \mathrm{mM}$ & $\mathrm{K} 4 \mathrm{Fe}(\mathrm{CN}) 6$ \\
\hline & $2 \mathrm{mM}$ & $\mathrm{MgCl} 2$ \\
\hline & $0,02 \%(\mathrm{v} / \mathrm{v})$ & NP-40 \\
\hline & $0,01 \%(\mathrm{w} / \mathrm{v})$ & Natrium Deoxycholat \\
\hline & $500 \mu \mathrm{g} / \mathrm{ml}$ & $\mathrm{X}-\mathrm{Gal}$ \\
\hline & & 1x PBS \\
\hline LacZ-buffer & $2 \mathrm{mM}$ & $\mathrm{MgCl} 2$ \\
\hline
\end{tabular}




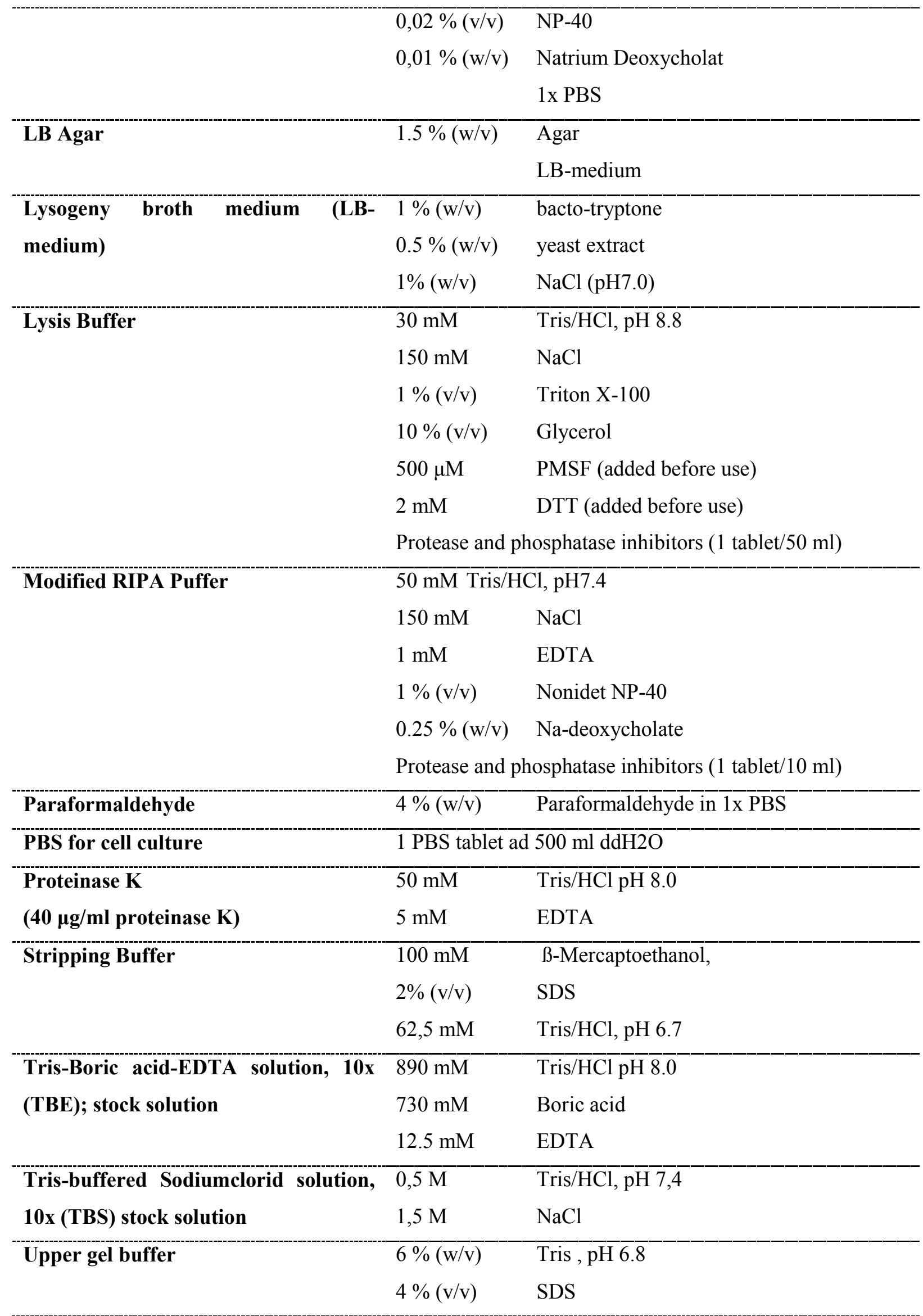




\subsection{Inhibitors}

The stock concentrations as well as the solvents are listed in Table 6 .

Table 6: List of inhibitors and their stock concentrations

\begin{tabular}{lllll}
\hline Inhibitor & Solvent & Stock concentration & Storage & Supplier \\
\hline HhAntag & DMSO & $10 \mathrm{mM}$ & $-20^{\circ} \mathrm{C}$ & Genentech \\
PI103 & DMSO & $3 \mathrm{mM}$ & $-20^{\circ} \mathrm{C}$ & Alexis \\
UO126 & DMSO & $10 \mathrm{mM}$ & $-20^{\circ} \mathrm{C}$ & Cell Signaling \\
\hline
\end{tabular}

\section{7. siRNA}

The siRNAs used in this thesis is listed in Table 7.

Table 7: List of siRNA and their stock concentrations

\begin{tabular}{lllll}
\hline siRNA & Solvent & Stock concentration & Storage & Supplier \\
\hline siDYRK1B_1 & $\mathrm{ddH}_{2} \mathrm{O}$ & $5 \mu \mathrm{M}$ & $-20^{\circ} \mathrm{C}$ & Dharmacon D-004806-01 \\
siDYRK1B_2 & $\mathrm{ddH}_{2} \mathrm{O}$ & $5 \mu \mathrm{M}$ & $-20^{\circ} \mathrm{C}$ & Dharmacon D-004806-02 \\
\hline
\end{tabular}

\subsection{Media}

\subsubsection{Media and agar plates for bacterial culturing}

Bacterial (E.coli) were cultured in Lysogeny broth medium and agar was prepared as described in Table 4. The LB medium/agar was autoclaved, cooled to $55^{\circ} \mathrm{C}$ and $50 \mu \mathrm{g} / \mathrm{ml}$ Ampicillin (Carl Roth GmbH, Karlsruhe) or $25 \mu \mathrm{g} / \mathrm{ml}$ Kanamycin (Carl Roth GmbH, Karlsruhe) were added. The plates and the media were stored at $4{ }^{\circ} \mathrm{C}$. 


\subsubsection{Media and reagents for cultivation of eukaryotic cell lines}

The media and reagents used for the culturing of eukaryotic cell lines are listed in Table 8 .

Table 8: List of supplementary materials used for the eukaryotic cells

\begin{tabular}{|c|c|}
\hline Medium or reagent & Supplier \\
\hline Accutase & PAA Laboratories GmbH, Pasching \\
\hline Dulbecco's Modified Eagle Medium (DMEM) & Gibco, Invitrogen GmbH, Karlsruhe \\
\hline Fetal calf serum (FCS) & Gibco, Invitrogen $\mathrm{GmbH}$, Karlsruhe \\
\hline HyperFect & \\
\hline $\begin{array}{l}\text { Penicillin (10.000 U/ml)/streptomycin } \\
\mathrm{mg} / \mathrm{ml})(\mathbf{P} / \mathrm{S})\end{array}$ & PAN Biotech GmbH, Aidenbach \\
\hline Puromycin dihydrochloride $(10 \mathrm{mg} / \mathrm{ml})$ & Sigma-Aldrich Chemistry GmbH, Steinheim \\
\hline RPMI 1640 & Gibco, Invitrogen $\mathrm{GmbH}$, Karlsruhe \\
\hline Trypsin/EDTA and TrypLE Express & Gibco, Invitrogen $\mathrm{GmbH}$, Karlsruhe \\
\hline
\end{tabular}

\subsection{Biological materials}

\subsubsection{Bacterial strains and growth}

In this thesis, E.coli stain DH5a (Invitrogen $\mathrm{GmbH}$, Karlsruhe) was used for transformation and amplification of plasmid DNA. Until use the competent cells were stored at $-80{ }^{\circ} \mathrm{C}$. The competent cells were maximally defrosted for 3 times and then thrown away.

\subsubsection{Eukaryotic cell lines}

All cell lines were cultured at $37^{\circ} \mathrm{C}, 5 \% \mathrm{CO}_{2}$ and $95 \%$ humidity in an incubator. The media was changed all 3-4 days and when the cells reached $80-90 \%$ confluence they were passaged. Dulbecco's Modified Eagle Medium (DMEM) or RPMI were used. The conditions for each cell line are given in Table 9. 
Table 9: Culture conditions for the used cell lines

\begin{tabular}{|c|c|c|c|c|}
\hline Cell line & Description & Media & Supplement & Reference \\
\hline HEK293 & $\begin{array}{l}\text { Human embryonic kidney } \\
\text { cell line }\end{array}$ & DMEM & $10 \% \mathrm{FCS}, 1 \% \mathrm{P} / \mathrm{S}$ & $\begin{array}{l}\text { ATCC (cat\# } \\
\text { CRL-1573) }\end{array}$ \\
\hline НЕК293SHН & $\begin{array}{l}\text { Shh-N secreting HEK } \\
\text { Cells }\end{array}$ & DMEM & $\begin{array}{l}10 \% \text { FCS, } 1 \% \mathrm{P} / \mathrm{S} \\
0.4 \mathrm{mg} / \mathrm{ml} \mathrm{G} 418\end{array}$ & $\begin{array}{l}\text { (Chen et al. } \\
\text { 2002) }\end{array}$ \\
\hline RMS-13 & $\begin{array}{l}\text { Alveolar RMS cell line, } \\
\text { wtRAS }\end{array}$ & RPMI & $10 \% \mathrm{FCS}, 1 \% \mathrm{P} / \mathrm{S}$ & $\begin{array}{l}\text { ATCC (cat\# } \\
\text { CRL-2061) }\end{array}$ \\
\hline $\begin{array}{l}\text { RMS-13 } \\
\text { pMSCVpuro }\end{array}$ & $\begin{array}{l}\text { RMS-13 stably transduced } \\
\text { with empty pMSCVpuro } \\
\text { vector }\end{array}$ & RPMI & $\begin{array}{l}10 \% \mathrm{FCS}, 1 \% \mathrm{P} / \mathrm{S}, \\
0.5 \mu \mathrm{g} / \mathrm{ml} \text { Puromycin }\end{array}$ & this work \\
\hline $\begin{array}{l}\text { RMS-13 } \\
\text { pMSCVpuro } \\
\text { NRAS12V }\end{array}$ & $\begin{array}{l}\text { RMS-13 stably transduced } \\
\text { with oncNRAS }\end{array}$ & RPMI & $\begin{array}{l}10 \% \mathrm{FCS}, 1 \% \mathrm{P} / \mathrm{S}, \\
0.5 \mu \mathrm{g} / \mathrm{ml} \text { Puromycin }\end{array}$ & this work \\
\hline $\begin{array}{l}\text { RMS-13 } \\
\text { pMSCVpuro } \\
\text { KRAS12V }\end{array}$ & $\begin{array}{l}\text { RMS-13 stably transduced } \\
\text { with oncKRAS }\end{array}$ & RPMI & $\begin{array}{l}10 \% \mathrm{FCS}, 1 \% \mathrm{P} / \mathrm{S}, \\
0.5 \mu \mathrm{g} / \mathrm{ml} \text { Puromycin }\end{array}$ & this work \\
\hline $\begin{array}{l}\text { RMS-13 } \\
\text { pMSCVpuro } \\
\text { HRAS12V }\end{array}$ & $\begin{array}{l}\text { RMS-13 stably transduced } \\
\text { with oncHRAS }\end{array}$ & RPMI & $\begin{array}{l}10 \% \mathrm{FCS}, 1 \% \mathrm{P} / \mathrm{S}, \\
0.5 \mu \mathrm{g} / \mathrm{ml} \text { Puromycin }\end{array}$ & this work \\
\hline RUCH-2 & $\begin{array}{l}\text { Embryonal RMS cell line, } \\
\text { wtRAS }\end{array}$ & DMEM & $10 \% \mathrm{FCS}, 1 \% \mathrm{P} / \mathrm{S}$ & $\begin{array}{l}\text { (Scholl et al. } \\
\text { 2000) }\end{array}$ \\
\hline $\begin{array}{l}\text { RUCH-2 } \\
\text { pMSCVpuro }\end{array}$ & $\begin{array}{l}\text { RUCH-2 stably transduced } \\
\text { with empty pMSCVpuro } \\
\text { vector }\end{array}$ & DMEM & $\begin{array}{l}10 \% \mathrm{FCS}, 1 \% \mathrm{P} / \mathrm{S}, \\
8 \mu \mathrm{g} / \mathrm{ml} \text { Puromycin }\end{array}$ & this work \\
\hline $\begin{array}{l}\text { RUCH-2 } \\
\text { pMSCVpuro } \\
\text { NRAS12V }\end{array}$ & $\begin{array}{l}\text { RUCH-2 stably transduced } \\
\text { with oncNRAS }\end{array}$ & DMEM & $\begin{array}{l}10 \% \mathrm{FCS}, 1 \% \mathrm{P} / \mathrm{S}, \\
8 \mu \mathrm{g} / \mathrm{ml} \text { Puromycin }\end{array}$ & this work \\
\hline $\begin{array}{l}\text { RUCH-2 } \\
\text { pMSCVpuro } \\
\text { KRAS12V }\end{array}$ & $\begin{array}{l}\text { RUCH-2 stably transduced } \\
\text { with oncKRAS }\end{array}$ & DMEM & $\begin{array}{l}10 \% \mathrm{FCS}, 1 \% \mathrm{P} / \mathrm{S}, \\
8 \mu \mathrm{g} / \mathrm{ml} \text { Puromycin }\end{array}$ & this work \\
\hline $\begin{array}{l}\text { RUCH-2 } \\
\text { pMSCVpuro } \\
\text { HRAS12V }\end{array}$ & $\begin{array}{l}\text { RUCH-2 stably transduced } \\
\text { with oncHRAS }\end{array}$ & DMEM & $\begin{array}{l}10 \% \mathrm{FCS}, 1 \% \mathrm{P} / \mathrm{S}, \\
8 \mu \mathrm{g} / \mathrm{ml} \text { Puromycin }\end{array}$ & this work \\
\hline
\end{tabular}




\subsubsection{Mouse lines}

The genetically manipulated mouse lines used in this work are listed in Table 10. Most mice were on an inbred C57BL/6 and BALB/c (Charles River Laboratories) background. Raising and breeding of animals and animal experimental methods are described in chapter 4.17.

Table 10: List of mouse lines

\begin{tabular}{|c|c|c|c|}
\hline Mouse lines & Background & Genetic modification & Literature \\
\hline Ptch $^{\text {del/+ }}$ & $\mathrm{Balb} / \mathrm{c}$ & Ptch deletion (knockout) & (Uhmann et al. 2007) \\
\hline $\begin{array}{l}\text { NRAS LSL-G12D } \\
f l /-\end{array}$ & $\mathrm{C} 57 \mathrm{BL} / 6$ & $\begin{array}{l}\text { Integration of a conditional } \\
\text { NRasG12D allele in the NRas } \\
\text { locus }\end{array}$ & (Haigis et al. 2008) \\
\hline $\begin{array}{l}L S L-K- \\
R A S G 12 D^{f /-}\end{array}$ & $\mathrm{C} 57 \mathrm{BL} / 6$ & $\begin{array}{l}\text { Integration of a conditional } \\
\text { KRasG12D allele in the KRas } \\
\text { locus }\end{array}$ & (Tuveson et al. 2004) \\
\hline FR-HRASG12V $V^{f l /-}$ & $\mathrm{C} 57 \mathrm{BL} / 6$ & $\begin{array}{l}\text { Integration of a conditional } \\
\text { HRasG12V allele in the HRas } \\
\text { locus }\end{array}$ & (Chen et al. 2009) \\
\hline Myf5creERT $T^{g /+}$ & $\begin{array}{ll}\text { Mixed } & \text { Balb/c, } \\
\text { C57BL/6, } & \text { SV/129 } \\
\text { and FVB/N } & \end{array}$ & $\begin{array}{l}\text { Targeted insertion of cre } \\
\text { cDNA into the endogenious } \\
\text { Myf5 locus }\end{array}$ & (Biressi et al. 2013) \\
\hline NMRI-Foxn1nu & outbred & Deletion of the FOXN1 gene & $\begin{array}{l}\text { The Jackson } \\
\text { Laboratory, Baltimore }\end{array}$ \\
\hline ROSA26-R fll- $^{f(1)}$ & C57BL/6 & $\begin{array}{l}\text { Integration of a conditional } \beta \text { - } \\
\text { galactosidase allele in the } \\
\text { ROSA26-locus }\end{array}$ & (Soriano 1999) \\
\hline
\end{tabular}

\subsection{DNA Oligonucleotides}

The DNA oligonucleotides were obtained from Eurofins MWG Operon, Ebersberg. They were adjusted to a stock concentration of $100 \mu \mathrm{M}$ with DNAse and RNAse-free water. The working concentration for PCR (see section 4.15.5) was $10 \mu \mathrm{M}$. The DNA oligonucleotides used are listed in Table 11, Table 12 and Table 13. 
Table 11: DNA-oligonucleotides (primers) for mouse genotyping and recombination assay.

\begin{tabular}{|c|c|c|c|c|}
\hline Mouse lines & Primer name & $\begin{array}{l}\mathbf{A T} \\
{\left[{ }^{\circ} \mathbf{C}\right]}\end{array}$ & Primer Sequence (5'-3' orientation) & Literature \\
\hline \multirow[b]{2}{*}{$\operatorname{Ptch}^{\mathrm{del/}+}$} & mPtcdelNx-F & \multirow{2}{*}{60} & TTCATTGAACCTTGGGGAACATT & \multirow{2}{*}{$\begin{array}{l}\text { Dissertation } \\
\text { F. Nitzki }\end{array}$} \\
\hline & $\mathrm{mPtcNxR}$ & & CCGGTAGAATTAGCTTGAAGTTCCT & \\
\hline \multirow{2}{*}{ wtPtch } & mPTCNx_F & \multirow{2}{*}{60} & TGGTAATTCTGGGCTCCCGT & \multirow{2}{*}{$\begin{array}{l}\text { (Uhmann et } \\
\text { al. 2007) }\end{array}$} \\
\hline & mPTCwt_R & & TCAAGGAGCAGAGGCCCAA & \\
\hline \multirow{4}{*}{$\begin{array}{l}\text { NRas LSL- } \\
\text { G12D }^{f l /+}\end{array}$} & mNRas-WT-For & \multirow{4}{*}{68} & AGACGCGGAGACTTGGCGAGC & \multirow{4}{*}{$\begin{array}{l}\text { (Haigis et } \\
\text { al. 2008) }\end{array}$} \\
\hline & mNRas-WT-Rev & & GCTGGATCGTCAAGGCGCTTTTCC & \\
\hline & mNRas-Mut-Rev & & AGCTAGCCACCATGGCTTGAGTAAGT & \\
\hline & & & CTGCA & \\
\hline \multirow{3}{*}{$\begin{array}{l}\text { LSL-KRas } \\
\text { G12D }\end{array}$} & Kras-WT_UP1 & \multirow{3}{*}{55} & CACCAGCTTCGGCTTCCTATT & \multirow{3}{*}{$\begin{array}{l}\text { (Tuveson et } \\
\text { al. 2004) }\end{array}$} \\
\hline & Kras-URP_Lp1 & & AGCTAATGGCTCTCAAAGGAATGTA & \\
\hline & KrasG12Dmut_UP & & CCATGGCTTGAGTAAGTCTGC & \\
\hline \multirow{3}{*}{$\begin{array}{l}\text { FR-HRas } \\
\text { G12V }^{\mathrm{fl} /-}\end{array}$} & $\begin{array}{l}\text { eCreRASF } \\
\text { eCreRasR }\end{array}$ & 64 & $\begin{array}{l}\text { GCCATCCCTCGCGTTCCTGTAGTC } \\
\text { CCTGCCCCACCTGCCAATGAGAAG }\end{array}$ & \multirow{3}{*}{$\begin{array}{l}\text { (Chen et al. } \\
\text { 2009) } \\
\text { This work }\end{array}$} \\
\hline & mHRasG12VrelF1 & \multirow{2}{*}{65} & TGGGGCAGGAGCTCCTGGATT & \\
\hline & mHRasG12VrelR1 & & GGTGTTGTTGATGGCAAATAC & \\
\hline \multirow{2}{*}{$\begin{array}{l}\text { Myf5creER } \\
T^{T^{g / 4}}\end{array}$} & $\mathrm{c}-\mathrm{R}$ & \multirow{2}{*}{58} & CCCGGCAAAACAGGTAGTTA & \multirow{2}{*}{$\begin{array}{l}\text { (Biressi et } \\
\text { al. 2013) }\end{array}$} \\
\hline & $\mathrm{c}-\mathrm{F}$ & & GCATTTCTGGGGATTGCTTA & \\
\hline \multirow{2}{*}{$w t M y f 5$} & cK382 & \multirow{2}{*}{59} & ACCCTCCAGCTCCAGACTTATC & \multirow{2}{*}{$\begin{array}{l}\text { (Biressi et } \\
\text { al. 2013) }\end{array}$} \\
\hline & cK383 & & CCCTGTAATGGATTCCAAGCTG & \\
\hline ROSA26- & Rosal & \multirow{3}{*}{56} & AAAGTCGCTCTGAGTTGTTAT & (P (Soriano \\
\hline \multirow[t]{2}{*}{$\boldsymbol{R}^{f l /+}$} & Rosa2 & & GCGAAGAGTTTGTCCTCAACC & \multirow[t]{2}{*}{ 1999) } \\
\hline & Rosa3 & & GGAGCGGGAGAAATGGATATG & \\
\hline
\end{tabular}

$\mathrm{AT}=$ Annealingtemperature 
Table 12: DNA-oligonucleotides (primers) for quantitative RT PCR

\begin{tabular}{|c|c|c|c|}
\hline $\begin{array}{l}\text { Analyzed } \\
\text { transcript }\end{array}$ & Primer name & Primer Sequence (5'-3' orientation) & $\begin{array}{l}\text { Supplier of } \\
\text { SYBRGreen }\end{array}$ \\
\hline \multirow[t]{2}{*}{$18 \mathrm{r} R \mathrm{R} A$} & $18 \mathrm{~s}$ forw & CGCAAATTACCCACTCCCG & Invitrogen \\
\hline & $18 \mathrm{~s}$ rev2 & TTCCAATTACAGGGCCTCGAA & and Quiagen \\
\hline \multirow[t]{2}{*}{ hDESMIN } & Desmin_RT_F1 & CATCGCGGCTAAGAACATTT & Quiagen \\
\hline & Desmin_RT_R1: & GCCTCATCAGGGAATCGTTA & \\
\hline \multirow[t]{2}{*}{ hDYRK1B } & hDYRK1B_F1 & TTGGCCAGGTGGTGAAAGCCTATGA & Invitrogen \\
\hline & hDYRK1B_R1 & CAATCTGGGCCTGGTTCAGGAAAGC & \\
\hline \multirow[t]{2}{*}{ hGLII } & HsaGli1 tq F & AGCTACATCAACTCCGGCCA & Invitrogen \\
\hline & HsaGli1 tq R & GCTGCGGCGTTCAAGAGA & \\
\hline \multirow[t]{2}{*}{ mGli1 } & mGli1-tq-f & TACATGCTGGTGGTGCACATG & Quiagen \\
\hline & mGli1-tq-r & ACCGAAGGTGCGTCTTGAGG & \\
\hline \multirow[t]{2}{*}{ mGli2 } & Gli2-RT-RCR-F & GGTCATCTACGAGACCAACTGC & Quiagen \\
\hline & Gli2-RT-RCR-R & GTGTCTTCAGGTTCTCCAGGC & \\
\hline \multirow[t]{2}{*}{ mGli3 } & Gli3F2 & GAAGGAACAACCCTAGTCAAGGAGGA & Invitrogen \\
\hline & Gli3-sybrgree R & CCAGCGGCACACGAACTCCTTCT & \\
\hline \multirow[t]{2}{*}{ mMyoD } & MyoD-Scerj-F & CCCCGGCGGCAGAATGGCTACG & Quiagen \\
\hline & MyoD-Scerj-R & GGTCTGGGTTCCCTGTTCTGT & \\
\hline \multirow[t]{2}{*}{ hMYOD } & hMYOD F & CGAACCCCCAACCCGATA & Invitrogen \\
\hline & hMYOD R & GAAAAAACCGCGCTGTG & \\
\hline \multirow[t]{2}{*}{$m M y f 5$} & mMyf5F.1 & TGACGGCATGCCTGAATGTAA & Invitrogen \\
\hline & mMyf5R.1 & CCAAGCTGGACACGGAGCT & \\
\hline \multirow[t]{2}{*}{ mMyogenin } & Myogenin-Scerj-F & GCAATGCACTGGAGTTCG & Invitrogen \\
\hline & Myogenin-Scerj-R & ACGATGGACGTAAGGGAGTG & \\
\hline \multirow[t]{2}{*}{ mPtch } & mPTC10 & TACAGTCCGGGACAGCATACC & Quiagen \\
\hline & mPTC22R & TCTCCTCACATTCCACGT & \\
\hline \multirow[t]{2}{*}{ hSHH } & hSHH_forw & GATGACTCAGAGGTGTAAGGAC & Quiagen \\
\hline & hSHH_rev & CCTCGTAGTGCAGAGACTCC & \\
\hline
\end{tabular}


Table 13: DNA-oligonucleotides (primers) for cloning and plasmid identification

\begin{tabular}{|c|c|c|}
\hline Application & Primer name & Primer Sequence (5'-3' orientation) \\
\hline $\begin{array}{l}\text { PCR-based amplification } \\
\text { of oncHRAS } \text { sequence } \\
\text { from pBabe puro } \\
\text { HRasV12 } \\
\text { plasmid }\end{array}$ & $\begin{array}{l}\text { HRasKlon- } \\
\text { XhoI-For } \\
\text { HRasKlon- } \\
\text { EcoRI-Rev }\end{array}$ & AGTCTCTCGAGAGTGTGGTGGTCAGCTTGGG \\
\hline $\begin{array}{l}\text { PCR-based amplification } \\
\text { of oncNRAS sequence } \\
\text { from pCaggs-NrasG12V }\end{array}$ & $\begin{array}{l}\text { NRasKlon- } \\
\text { XhoI-For3 } \\
\text { NRasKlon- } \\
\text { EcoRI-Rev3 }\end{array}$ & $\begin{array}{l}\text { AGTCTCTCGAGATGACTGAGTACAAACTGGT } \\
\text { AGCTCTGAATTCTTACATCACCACACATGGCA }\end{array}$ \\
\hline $\begin{array}{l}\text { PCR-based amplification } \\
\text { of oncKRAS sequence } \\
\text { from KRAS12V plasmid }\end{array}$ & $\begin{array}{l}\text { KRasKlon- } \\
\text { XhoI-For3 } \\
\text { KRasKlon- } \\
\text { HpaI-Rev3 }\end{array}$ & $\begin{array}{l}\text { AGTCTCTCGAGCTTTTGGAGTACGTCTTTAGG } \\
\text { AGCTCTGTTAACTTACATAATTACACACTTTG }\end{array}$ \\
\hline Sanger sequencing & $\begin{array}{l}\text { pMSCVpuro- } \\
\text { seq-F } \\
\text { pMSCVpuro- } \\
\text { seq-R } \\
\text { pNRasID-Rev } \\
\text { pKRasID-Rev } \\
\text { pHRasID-Rev }\end{array}$ & $\begin{array}{l}\text { GCCTTCGCCTGTCCTCATGTATTG } \\
\text { CCTCATGTACTGGTCCCTCATT } \\
\text { TCATCCGAGTCCTTCACCCGTT }\end{array}$ \\
\hline
\end{tabular}




\subsection{Antibodies}

For immunohistochemistry (IHC) and Western blot (WB) analysis the following antibodies were used with the specified conditions (see Table 14 and Table 15).

Table 14: List of primary antibodies

\begin{tabular}{|c|c|c|c|}
\hline Antibody & Method & Dilution & Diulent \\
\hline pAb mouse anti-Akt, BD Pharmingen & WB & 1:1.000 & $2 \% \mathrm{BSA} / 0.2 \%$ Azide in TBST \\
\hline pAb rabbit anti-ERK, Sigma-Aldrich & WB & $1: 4000$ & $2 \% \mathrm{BSA} / 0.2 \%$ Azide in TBST \\
\hline mAb mouse anti-HSC70, Santa Cruz & WB & $1: 10.000$ & $2 \% \mathrm{BSA} / 0.2 \%$ Azide in TBST \\
\hline mAb mouse anti-Ki-67, BD Pharmingen & $\mathrm{IHC}$ & $1: 50$ & $0.02 \%$ casein in TBS \\
\hline pAb rabbit anti-Ras, Cell Signaling & WB & $1: 1000$ & $2 \%$ BSA $/ 0.2 \%$ Azide in TBST \\
\hline mAb mouse anti-p-AKT, Cell Signaling & WB & 1:1.000 & $2 \% \mathrm{BSA} / 0.2 \%$ Azide in TBST \\
\hline pAb rabbit anti-p-ERK, Cell Signaling & WB & $1: 2.000$ & $2 \%$ BSA $/ 0.2 \%$ Azide in TBST \\
\hline
\end{tabular}

Table 15: List of secondary antibodies

\begin{tabular}{|c|c|c|c|}
\hline Secondary antibodies & Method & Dilution & Diluent \\
\hline En vision+ anti- rabbit/mouse/HRP, Dako & $\mathrm{IHC}$ & undiluted & \\
\hline rabbit anti rat/biotinylated, Dako & $\mathrm{IHC}$ & $1: 100$ & $0.02 \%$ casein in TBS \\
\hline $\begin{array}{l}\text { pAb goat anti-rabbit IgG, HRP- } \\
\text { conjugated, Sigma-Aldrich }\end{array}$ & WB & $1: 5000$ & $\begin{array}{l}5 \%(\mathrm{w} / \mathrm{v}) \text { milkpowder in TBS- } \\
0.1 \% \text { tween- } 20\end{array}$ \\
\hline $\begin{array}{l}\text { pAb sheep anti- mouse/HRP-conjugated, } \\
\text { GE Healthcare }\end{array}$ & WB & $1: 5000$ & $\begin{array}{l}5 \%(\mathrm{w} / \mathrm{v}) \text { milkpowder in TBS- } \\
0.1 \% \text { tween }-20\end{array}$ \\
\hline Streptavidin/HRP, Dako & $\mathrm{IHC}$ & $1: 1000$ & TBS \\
\hline
\end{tabular}




\subsection{Plasmids}

The Plasmids used and generated in this work are listed in Table 16.

Table 16: List of Plasmids

\begin{tabular}{|c|c|c|}
\hline Name & Application & Supplier/Reference \\
\hline pCaggs-NrasG12V & $\begin{array}{l}\text { Amplification of NRAS12V for cloning } \\
\text { into pMSCVpuro }\end{array}$ & (Rudalska et al. 2014) \\
\hline$K R A S 12 V$ & $\begin{array}{l}\text { Amplification of KRAS } 12 \mathrm{~V} \text { for cloning } \\
\text { into pMSCVpuro }\end{array}$ & $\begin{array}{l}\text { obtained from Matthias Lauth } \\
\text { (Lauth et al. 2010) }\end{array}$ \\
\hline pBabe puro HRas & $\begin{array}{l}\text { Amplification of HRAS } 12 \mathrm{~V} \text { for cloning } \\
\text { into } p M S C V \text { puro }\end{array}$ & Addgene \\
\hline pMSCVpuro & $\begin{array}{l}\text { Backbone for pMSCVpuro oncRAS } \\
\text { variants }\end{array}$ & $\begin{array}{l}\text { Clontech Laboratories, Inc., } \\
\text { Mountain View, USA }\end{array}$ \\
\hline pMSCVpuro-NRAS12V & $\begin{array}{l}\text { Stable expression of NRAS12V in RMS } \\
\text { cell lines }\end{array}$ & this work \\
\hline pMSCVpuro-KRAS12V & $\begin{array}{l}\text { Stable expression of KRAS12V in RMS } \\
\text { cell lines }\end{array}$ & this work \\
\hline pMSCVpuro-HRAS12V & $\begin{array}{l}\text { Stable expression of HRAS12V in RMS } \\
\text { cell lines }\end{array}$ & this work \\
\hline
\end{tabular}




\subsection{Software}

The Software used in this work is listed in Table 17.

Table 17: List of Software used in this thesis:

\begin{tabular}{ll}
\hline Name & Developer/Reference \\
\hline ABI 3500 & Applied Biosystems, Darmstadt, Germany \\
Adobe Photoshop CS5 & Adobe Systems Incorporated, San Jose, USA \\
BD FACSDiva & Becton Dickinson GmbH, Heidelberg \\
BioEdit & Ibis Biosciences, Carlsbad, USA \\
Cell F & Olympus Europa GmbH, Hamburg, Germany \\
Endnote X5 & Thomson ISI ResearchSoft, California, USA \\
Fiji & (Schindelin et al. 2012) \\
GraphPad Prism 6 & GraphPad Software, Inc., La Jolla, CA, USA \\
Intas GDS & Intas Science Imaging Instruments GmbH, Göttingen \\
Gen5 1.11 & BioTek Instruments, Inc., Bad Friedrichshall \\
Microsoft Office & Microsoft Co., Redmont, USA \\
SDS 2.2 & Applied Biosystems, Darmstadt \\
SnapGene & GSL Biotech (snapgene.com) \\
\hline
\end{tabular}

\subsection{Cell biology methods}

\subsubsection{Cryoconservation of cells}

For cryconservation the cells were grown up to $80 \%$ confluency, washed with PBS and incubated for $2 \mathrm{~min}$ with $2 \mathrm{ml}$ TriplExpress ${ }^{\circledR}$ to detach. After that the reaction was stopped with $8 \mathrm{ml}$ FCS containing media. The cells were transferred to a $15 \mathrm{ml}$ tube and centrifuged for $5 \mathrm{~min}$ at $300 \mathrm{~g}$. The pellets were resuspended in 5\% DMSO containing FCS and aliquoted in $1 \mathrm{ml}$ portions into cryotubes. The cryotubes were stored overnight at $-80^{\circ} \mathrm{C}$ in a freezing container and then transferred to a liquid nitrogen tank.

\subsubsection{Stable retroviral transduction of RMS cell lines}

Virus particles were generated with the packaging cell line Platinum-E. This was performed in collaboration with Dr. Michael Engelke, Institute of Cellular and Molecular Immunology. 
For this purpose Platinum-E were grown to approximately $70 \%$ confluency in $5 \mathrm{~cm}$ culture dishes and incubated overnight in culture medium. The next day the cells were transfected for $30 \mathrm{~min}$ at RT by adding drop wise the transfection mixture to the dish while shaking. The transfection mixture contained $400 \mu \mathrm{l}$ serum free medium, $9 \mu 1$ TransIT ${ }^{\circledR}-\mathrm{LT} 1$ Transfection Reagent and $3 \mu \mathrm{g}$ of the retroviral expression vectors pMSCVpuro, pMSCVpuro-NRAS12V, pMSCVpuro-KRAS12V or pMSCVpuropuro-HRAS12V. After transfection the cells were washed with PBS and incubated for $48 \mathrm{~h}$ with $4 \mathrm{ml}$ fresh culture medium (DMEM 2\% FCS, $1 \% \mathrm{P} / \mathrm{S})$. After that the virus containing supernatant was sterile-filtered by using a $0.45 \mu \mathrm{m}$ pore sterile filter and used for the stable transduction.

The stable transduction of the RMS cell lines was performed in collaboration with Tobias Puckrop, Institute of Hematology and Oncology. For this purpose the cells were grown to 70$80 \%$ confluency, washed with PBS, detached and counted. $1 \times 10^{5}$ cells were seeded in a 6well plate and $100 \mu 1$ of the virus-containing supernatant (virus particle with the expression plasmids pMSCVpuro, pMSCVpuro-NRAS12V, pMSCVpuro-KRAS12V or pMSCVpuro$H R A S 12 \mathrm{~V}$ ) were added. The incubation with the respective virus particles was performed in duplicates. Next, the plate was centrifuged for $1 \mathrm{~h}, 2000 \mathrm{rpm}$ at RT, followed by incubation at $37^{\circ} \mathrm{C}$ overnight. During the next 3 days the media with the virus particles were refreshed daily. After 3 days the cells containing the plasmid were selected by culturing with culture media containing puromycin.

\subsubsection{Generation of conditioned medium}

In order to generate conditioned medium the cells were grown to a density of $70 \%$, washed with PBS and incubated for $24 \mathrm{~h}$ in DMEM containing $2 \% \mathrm{FCS}$ and $1 \% \mathrm{P} / \mathrm{S}$. After that time the supernatant was sterile-filtered $(0.2 \mu \mathrm{m}$ pore size $)$ and stored for up to 3 month at $4{ }^{\circ} \mathrm{C}$.

\subsection{4. $\quad$ SHH secretion analysis}

For the identification whether cells were able to secrete SHH, B9 cells were incubated with conditioned medium of the respective cell line. For this purpose 1 x $10^{5} \mathrm{~B} 9$ cells were seeded onto a 6-well plate and incubated overnight. The next day the medium was replaced by the conditioned medium (see chapter 4.14.3). As positive control the conditioned medium from SHH secreting HEK293SHH cells was used. After $48 \mathrm{~h}$ the RNA was isolated, reversed transcribed and the $18 S$ and Glil expression were measured by qRT-PCR. 


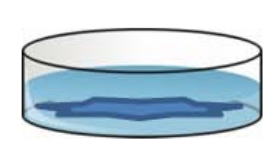

Grow the cells to be tested in medium
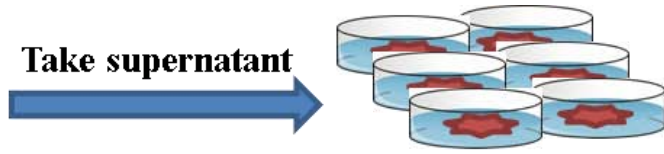

Incubate the reporter

cells (B9) with the

supernatant

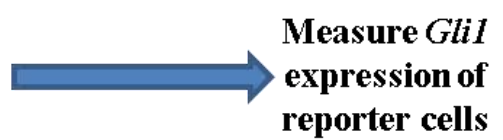

reporter cells

Figure 3: Experimental setting for the SHH secretion analysis. The cells to be tested were grown in medium as described in chapter 4.14.3, the supernatant was taken, filtered and transferred to the reporter cells B9. After $48 \mathrm{~h}$ the cells were harvested and used for gene expression analysis.

\subsubsection{Metabolic activity assay and cell proliferation assay}

To test metabolic activity of human cell lines, WST-1 reagent from Roche was used. For this purpose 8000 cells were seeded in a 96-well-platte and incubated overnight. The next day the cells were incubated for $24 \mathrm{~h}$ with the desired inhibitors or solvents. The incubation was stopped $3 \mathrm{~h}$ before the end of treatment, by replacement of the drug-containing medium with fresh media containing $100 \mu \mathrm{l}$ of WST- 1 reagent $\left(1: 10\right.$ dilution) at $37{ }^{\circ} \mathrm{C}$ for $3 \mathrm{~h}$. During the incubation with the WST-1 reagent, the tetrazolium salt WST-1 is cleaved to a soluble formazan dye by cellular mitochondrial dehydrogenases of viable cells. Thus, the amount of formazan dye is proportional to the amount of living cells and can be quantified by measuring the absorbance using a spectrophotometer at a wavelength of $450 \mathrm{~nm}$.

To test proliferation of human cell lines the chemiluminescence based Cell Proliferation BrdU ELISA Kit from Roche was used as described by the manufacturer's instruction. This assay is based on the principal that during replication the pyrimidinanalog Bromdeoxyuridin (BrdU) replaced thymidine residues in the DNA. Therefore the amount of incorporated BrdU is proportional to the amount of proliferating cells. For the assay 8000 cells were seeded in a 96well-platte. The next day the cells were incubated for $24 \mathrm{~h}$ with the desired inhibitors or solvents. $10 \mu \mathrm{M}$ BrdU-reagent was added to the media for the last $22 \mathrm{~h}$. Then the cells were fixed, denaturated and labeled with anti-BrdU-POD for $1 \mathrm{~h}$. After a washing step, the peroxidase substrate was added and BrdU incorporation was measured by a luminometer. The resulting luminescence is proportional to the proliferation of cells.

\subsection{6. $\quad$ DYRK1B knockdown in RMS cells lines}

For knockdown of DYRK1B two specific siRNAs i.e. DYRK1B_01 and DYRK1B_02 siRNA from Dharmacon (D-004806-01 and D-004806-02) were used. For this purpose 
$2 \times 10^{5}$ cells were seeded onto a 6 -well plate in a final volume of $2.4 \mathrm{ml}$. Next the siRNA mixture containing $4 \mu 1$ siRNA ( $2 \mu 1$ of DYRK1B_01 [5 $\mu \mathrm{M}]$ and $2 \mu 1$ DYRK1B_02 [5 $\mu \mathrm{M}]$ ) or solvent, $12 \mu 1$ HiPerFect reagent and $82 \mu 1$ media containing FSC was added to the cells and incubated for $48 \mathrm{~h}$. After incubation the RNA was isolated, reverse transcribed and the $18 S, G L I 1$ and DYRK1B expression measured by qRT-PCR.

\subsection{Molecular biology methods}

\subsubsection{Genomic DNA isolation from murine mouse tail and tissue}

For the isolation of genomic DNA (gDNA) from mouse tails (mouse tail biopsy see chapter 4.17.2) or tissue a small peace ( $\sim 5-10 \mathrm{mg})$ was sliced and incubated with $500 \mu \mathrm{l}$ STE-Buffer and $25 \mu \mathrm{l}$ Proteinase $\mathrm{K}\left(10 \mathrm{mg} / \mathrm{ml}\right.$ stock) over night at $55^{\circ} \mathrm{C}$ in a $1.5 \mathrm{ml}$ tube. Undigested tissue was pelleted for $10 \mathrm{~min}$ at $13.000 \mathrm{rpm}$ and $400 \mu \mathrm{l}$ of the supernatant were transferred in a new $1.5 \mathrm{ml}$ tube with $1 \mathrm{ml}$ precooled $99.9 \%$ Ethanol. The tubes were shaken and centrifuged for $25 \mathrm{~min}$ at $13000 \mathrm{rpm}$ and $4{ }^{\circ} \mathrm{C}$. Pellets were washed with $70 \%$ Ethanol and dried for $10 \mathrm{~min}$ at $55^{\circ} \mathrm{C}$. After the incubation time the pellets were solved with $100 \mu \mathrm{l}$ Ampuwa water and stored at $4{ }^{\circ} \mathrm{C}$.

\subsubsection{Total RNA isolation with TRIzol ${ }^{\circledR}$}

For RNA isolation from cells the TRIzol ${ }^{\circledR}$ Reagent from the company Invitrogen was used according to the manufacturer's instruction. Briefly, the cells on the plate were washed once with PBS and solved with $1 \mathrm{ml}$ TRIzol ${ }^{\circledR}$. Unless stated otherwise, the following steps were performed on ice to prevent RNA degradation. The detached cells were transferred into a pre cooled $2 \mathrm{ml}$ tube and vortexed for $2 \mathrm{~min}$ at the highest level followed by an incubation of 5 min at RT. Afterwards, $200 \mu 1$ chloroform were added and vortexed at the highest level for 15 seconds. After 3 minute incubation the tubes were centrifuged for $10 \mathrm{~min}$ at $12000 \mathrm{rpm}$ and $4{ }^{\circ} \mathrm{C}$. Subsequently the upper phase, which contains the RNA, was transferred to a new precooled $2 \mathrm{ml}$ tube containing $500 \mu \mathrm{l}$ Isopropanol. After precipitation over night at $-20{ }^{\circ} \mathrm{C}$ the RNA was pelleted by centrifugation at $12000 \mathrm{rpm} 4{ }^{\circ} \mathrm{C}$ for $20 \mathrm{~min}$. The pellets were washed two times with $500 \mu 170 \%$ Ethanol, dried at room temperature (RT) for 5 min. and dissolved with 10-60 $\mu 1$ RNase-free $\mathrm{H}_{2} \mathrm{O}$ for $5-10 \mathrm{~min}$ at $56{ }^{\circ} \mathrm{C}$ depending on the pellet size and the used cell number. 


\subsubsection{RNA isolation from tissue with TRIzol ${ }^{\circledR}$}

The RNA isolation from tissues was performed by using the TRIzol ${ }^{\circledR}$ Reagent from the company Invitrogen according to the manufacturer's instruction. In short, approximately $20 \mathrm{mg}$ tissue was transferred in a $2 \mathrm{ml}$ tube containing $1 \mathrm{ml}$ TRIzol ${ }^{\circledR}$. The tissue was homogenized on ice with the homogenization tool at stage B-C for up to 1 minute followed by incubation for 5 min at RT. RNA isolation was done as described in chapter 4.15.2.

\subsubsection{Photometric quantification of total RNA by NanoDrop}

To determine the concentration of DNA and RNA, $1 \mu$ of the solution was added onto the lower optical surface slot at the NanoDrop and the concentration was measured by determining the optical density (OD). For calculation of the final concentration $(c=n g / \mu 1)$ the device uses the following formula.

$$
c=O D 260 \times 50(d s D N A) \text { or } 40(s s R N A)
$$

Additional to the $\mathrm{OD}_{260}$ the $\mathrm{OD}_{280}$ was measured to determine the amount of protein and to calculate the purity of the RNA or DNA. For this purpose the ratio of the $\mathrm{OD}_{260}$ and $\mathrm{OD}_{280}$ was calculated that should be between $1.8-2.0$ for DNA (purity of DNA $>90 \%$ ) and 2.0 for RNA (purity of RNA $>90 \%$ ).

\subsubsection{Polymerase chain reaction (PCR)}

For the amplification of double strand DNA molecules a polymerase chain reaction (PCR) was performed in a total volume of $10 \mu \mathrm{l}$. The conditions used for PCR varied depending on the DNA oligonucleotide, the amplified fragment size and the GC-content. The PCR starts with initial denaturation step for $5-10 \mathrm{~min}$ at $95{ }^{\circ} \mathrm{C}$ followed by $25-35$ cycles of denaturation (95 ${ }^{\circ} \mathrm{C}$ for $30-90$ seconds), annealing $\left(55-65^{\circ} \mathrm{C}\right.$ for $30-90$ seconds) and an elongation step ( $72{ }^{\circ} \mathrm{C}$ for 1-2 min depending on the fragment size). At the end an additional elongation step was performed at $72{ }^{\circ} \mathrm{C}$ for 3-7 min. For analysis of the PCR product the DNA was loaded to a $0.5-2 \%$ agarose gel in TBE buffer. 
Example for a typical PCR approach

$\begin{array}{ll}10-100 \mathrm{ng} & \text { DNA template } \\ 0.5 \mu \mathrm{M} & \text { specific Forward DNA-oligonucleotide } \\ 0.5 \mu \mathrm{M} & \text { specific Reverse DNA-oligonucleotide } \\ 0.2 \mathrm{mM} & \text { dNTP-mix } \\ 1 \%(\mathrm{v} / \mathrm{v}) & \text { Cresol solution } \\ 1 \mathrm{x} & \text { Taq-Polymerase-buffer } \\ 0.1 \mathrm{U} & \text { Taq-Polymerase }\end{array}$

\subsubsection{Agarose gel electrophoresis}

Agarose gel electrophoresis was performed in 0.5 to $2.0 \%(\mathrm{w} / \mathrm{v})$ agarose gels and TBE buffer. The percentage of the gel was dependent on the size of the DNA-/ or RNA fragment. For visualization of the fragments $0.2 \mu \mathrm{g} / \mathrm{ml}$ ethidium bromide was added to the gel. The fragments were separated in a TBE buffer filed gel electrophoresis chamber at a constant voltage of $100 \mathrm{~V}$. For the detection a UV-transilluminart (Intas, Göttingen) was used that visualizes the ethidium bromide stained fragments. The identification of the band size was done with the help of a DNA-ladder (100bp ladder).

\subsubsection{Reverse transcription}

Reverse transcription of RNA was done in a final volume of $20 \mu 1$. For this purpose $2 \mu \mathrm{g}$ RNA were incubated with $250 \mathrm{~g}$ hexameres at $70{ }^{\circ} \mathrm{C}$ for $10 \mathrm{~min}$. Afterwards, 1st strand Buffer (Invitrogen), $10 \mathrm{mM}$ DTT and $0.5 \mathrm{mM}$ dNTPs were added, followed by a 10 min incubation at RT. After pre-warming at $42{ }^{\circ} \mathrm{C}$ for $2 \mathrm{~min}, 50 \mathrm{U}$ reverse transcriptase (SuperScript II) were added to start the reaction. The reaction was stopped after $1 \mathrm{~h}$ at $70{ }^{\circ} \mathrm{C}$ for $10 \mathrm{~min}$. The cDNA was stored at $-20^{\circ} \mathrm{C}$. Assuming that the reverse transcription was $50 \%$ efficient the resulting cDNA has a concentration of $50 \mathrm{ng} / \mu \mathrm{l}$.

\subsubsection{Quantitative Real Time-PCR (qRT-PCR)}

For quantification of gene expression levels a SYBR-green based quantitative realtime PCR (qRT-PCR) assay was performed with the primer combinations given in Table 12. For the reaction $4 \mu \mathrm{l}$ qPCR SuperMix-UDG (Invitrogen) or QuantiTect SYBR-Green RT-PCR-Mix (Qiagen), $0.4 \mu \mathrm{M}$ forward and reverse primer and $100 \mathrm{ng}$ template cDNA were used in a final volume of $10 \mu \mathrm{l}$. The quantification of the expression of murine and human genes was calculated using the standard curve method. For this purpose each sample was measured in 
parallel to a corresponding standard. The standard curves were prepared by 1:5 serial dilutions of the standard sample. Based on the determined values of the standard curve, a linear trendline was generated. This was done by plotting the logarithm of the quantity against the $\mathrm{Ct}$-value for each dilution. Thus, the linear trendline follows the equation $\mathrm{y}=\mathrm{mx}+\mathrm{b}$. With this equation the quantity of each gene was determined.

At the end the expression of each gene was normalized to $18 S$ rRNA expression of the respective cDNA sample. To analyze the results the software's SDS 2.2.1 (Applied Biosystems) and Microsoft EXCEL (Microsoft Co) were used. Statistics and graphics were done with GraphPadPrism6.

\subsubsection{Cloning and sequencing techniques}

\subsubsection{Transformation of E.coli to amplify plasmid DNA}

For transformation competent E. coli DH5 $\alpha$ were thawed on ice, $100 \mu 1$ of the cells were mixed with 50-100 ng of plasmid DNA and incubated on ice for $20 \mathrm{~min}$. Next, the mixture was heat shocked at $42^{\circ} \mathrm{C}$ for 45 seconds followed by incubation for $2 \mathrm{~min}$ on ice. Afterwards, $500 \mu \mathrm{l}$ super optimal broth with catabolite repression (SOC) medium were added and the mixture was incubated for $1 \mathrm{~h}$ at $37^{\circ} \mathrm{C}$ and $900 \mathrm{rpm}$. At the end, $20-200 \mu \mathrm{l}$ of the cell suspension was plated onto lysogeny broth (LB) agar plates containing the adequate antibiotic as a selection marker and incubated overnight in an incubator at $37^{\circ} \mathrm{C}$. Single colonies were picked and used for inoculation of $1.5 \mathrm{ml} \mathrm{LB}$ medium at $37{ }^{\circ} \mathrm{C}$ overnight. This was followed by a small scale plasmid purification with the HiPure Plasmid DNA Purification Kit (Invitrogen $\mathrm{GmbH}$, Karlsruhe) according to the manufacturer's instruction. To perform a large scale plasmid purification $100 \mathrm{ml}$ LB medium were inoculated with $1 \mathrm{ml}$ of transformed E.coli cells and incubated overnight at $37^{\circ} \mathrm{C}$. The purification of plasmids was done with the

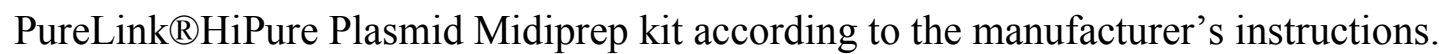

\subsubsection{Restriction digest}

Restriction digests of plasmids and PCR products were performed with appropriate enzymes and buffers according to the manufacturer's instructions. If necessary, a double restriction was performed. If the buffers were not compatible the DNA was precipitated after the first restriction reaction and subjected to the second restriction reaction. Each restriction reaction 
was carried out for $1 \mathrm{~h}$ at the optimal temperature for each enzyme. All used restriction enzymes used in this work were ordered from NEB (Ipswich, USA).

\subsubsection{Isolation of DNA fragments from agarose gels}

For isolation of DNA fragments from agarose gels, the fragments were loaded to a $1 \%$ agarose gel, separated and cut out using a sterile scalpel under $70 \% \mathrm{UV}$ light (to reduce the risk of UV-induced mutations). The DNA was purified with the DNA purification QIAquick Gel Extraction Kit (Quiagen) according to the manufacturer's instructions. The concentration of the purified DNA was calculated as described in chapter 4.15.4.

\subsubsection{Ligation}

To connect two pieces of DNA with compatible restriction ends a ligation T4 DNA Ligase system was used. For ligation the molecular ratio of the insert and vector was 3:1 (insert:vector). To calculate the molar ratio for the amount of the insert the following formula was used.

$$
\text { amount of insert }[\mathrm{ng}]=\frac{\text { insert length }[\mathrm{bp}] \times \text { amount of vector }[\mathrm{ng}]}{\text { vector length }[\mathrm{bp}] \times \text { molar ratio }\left(\frac{\text { insert }}{\text { vector }}\right)}
$$

Ligation was performed using an insert and vector mixture at a molar ratio of $3: 1$ in a total amount of 100 ng DNA. The DNA was incubated with 4 U T4 DNA Ligase in $1 \times$ T4 Ligase buffer in a final volume of $10 \mu \mathrm{l}\left(\mathrm{ad}\right.$ with $\left.\mathrm{ddH}_{2} \mathrm{O}\right)$ at $4^{\circ} \mathrm{C}$ for at least $48 \mathrm{~h}$. The reaction was stopped by heat-inactivation for $10 \mathrm{~min}$ at $65^{\circ} \mathrm{C}$ and the plasmids were transformed into E.coli (see chapter 4.15.9.1).

\subsubsection{Generation of RAS expression plasmids}

To generate the vectors pMSCVpuro-NRAS12V, pMSCVpuro-KRAS12V and pMSCVpuro$H R A S 12 \mathrm{~V}$, the respective RAS sequences were amplified from $p$ Caggs-NRASG12V, a KRAS plasmid and pBabe puro HRAS12V (addgene), by PCR. The primers used for the PCR are given in Table 13 and had overhangs containing specific restriction enzymes for cloning the fragments into pMSCVpuro. The PCR was done with the Phusion ${ }^{\circledR}$ High-Fidelity DNA Polymerase (NEB) in final reaction volume of $20 \mu \mathrm{l}$ according to the following protocol: 


$\begin{array}{lllll}50 \mathrm{ng} & \text { template plasmidDNA } & 1^{\circ} & 95^{\circ} \mathrm{C} \\ 0.5 \mu \mathrm{M} & \text { forward Primer } & 30^{\circ} & 95^{\circ} \mathrm{C} \\ 0.5 \mu \mathrm{M} & \text { reverse Primer } & 2.5^{\circ} & 60^{\circ} \mathrm{C} & 30 \text { cycles } \\ 0.2 \mathrm{mM} & \text { dNTP mix } & 5^{\circ} & 60^{\circ} \mathrm{C} & \\ 1 \mathrm{x} & \text { Phusion }{ }^{\circledR} \mathrm{GC} \text { Buffer Pack } & & \infty & 8^{\circ} \mathrm{C} \\ 2.5 \mathrm{mM} & \mathrm{MgCl} & & \\ 0.05 \mathrm{U} & \text { Phusion }{ }^{\circledR} \text { High-Fidelity } & \text { DNA } & & \\ & \text { Polymerase }\end{array}$

The amplified fragments were purified by agarose gel extraction and eluted in $30 \mu \mathrm{ddH} 2 \mathrm{O}$. The inserts were digested with XhoI and EcoRI (HRAS12V and NRAS12V) or XhoI and HpaI (KRAS12V) (see chapter4.15.9.2), separated on a $0.7 \%$ agarose gel, purified and cloned into the pMSCVpuro vector which was also digested with XhoI and EcoRI (HRAS12V and NRAS12V) or XhoI and HpaI (KRAS12V). For this purpose insert and the vector were mixed at a molar ratio of 3:1, respectively, and ligated using T4 DNA Ligase. The plasmids were transfected into E.coli DH5a and small and medium-scale plasmid purification was performed. For the final plasmids see attachment Figures Appendix 1, 2 und 3.

\subsubsection{DNA Sequencing}

Sanger sequencing using the BigDye ${ }^{\circledR}$ reagent was employed. For sequencing 20-200 ng plasmid DNA, 100 pmol sequencing primer (see Table 13), 1x BigDye 3.1 and 1x BigDye buffer reagent were mixed with $\mathrm{ddH}_{2} \mathrm{O}$ to a final volume of $10 \mu \mathrm{l}$ and the reaction was done in an $\mathrm{ABI} 3500 \mathrm{XL}$ sequencing device under the following conditions.

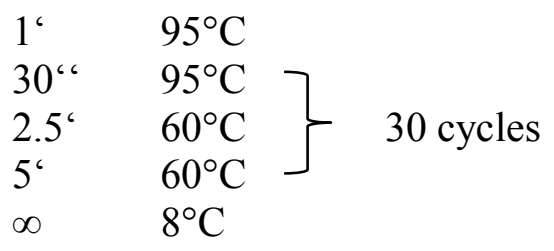

The resulting sequences and electropherograms were analyzed with the help of the BioEdit software. 


\subsection{Protein chemistry and histochemistry methods}

\subsubsection{Protein isolation from cells}

For the isolation of protein from cultured cells, the cells were removed with a cell scraper from the plate, transferred to a $15 \mathrm{ml}$ tubes and pelleted by centrifugation for $5 \mathrm{~min}$ at 2.000 rpm. The cell pellet was once washed with $700 \mu \mathrm{l}$ PBS and transferred to a $1.5 \mathrm{ml}$ tube. Afterwards the cells were pelleted, shock-frozen in liquid nitrogen and thawed on ice. The pellets were solved in 50-100 $\mu$ lysis buffer containing $500 \mu \mathrm{M}$ PMSF and $2 \mathrm{mM}$ DTT, and incubated on ice for $20 \mathrm{~min}$. Then the cell lysates were centrifuged at $13.000 \mathrm{rpm}$ for $25 \mathrm{~min}$ at $4{ }^{\circ} \mathrm{C}$. The supernatant was transferred to a new $1.5 \mathrm{ml}$ tube and the protein concentration was measured by Pierce ${ }^{\circledR}$ BCA Protein Assay Kit according to manufacturer's instructions. As standard bovine serum albumin was used. The protein lysates were stored at $-80{ }^{\circ} \mathrm{C}$.

\subsubsection{Protein isolation from tissue for Western Blot}

Approximately $20 \mathrm{mg}$ tissue was transferred in a $2 \mathrm{ml}$ tube containing $300 \mu \mathrm{L}$ modified RIPA buffer. The tissue was homogenized on ice with the homogenization tool at stage B-C for up to $1 \mathrm{~min}$, lysed for $10 \mathrm{~min}$ on ice and centrifuged at $13.000 \mathrm{rpm}$ for $25 \mathrm{~min}$ at $4{ }^{\circ} \mathrm{C}$. The supernatant was transferred to a new $1.5 \mathrm{ml}$ tube and the protein concentration was measured by Pierce ${ }^{\circledR}$ BCA Protein Assay Kit according to manufacturer's instructions. As standard Bovine serum albumin was used. The protein lysates were stored at $-80{ }^{\circ} \mathrm{C}$.

\subsubsection{Western blot}

To perform a western blot analysis, $30-50 \mu \mathrm{g}$ of total protein lysates were denatured at $96{ }^{\circ} \mathrm{C}$ for $5 \mathrm{~min}$ at $700 \mathrm{rpm}$ and $3 \mu \mathrm{l} 6 \mathrm{x}$ loading buffer was added. The proteins and a pre-stained protein standard (SeeBlue Plus2), to estimate the molecular weight of the proteins, were loaded to a NuPAGE Novex midi gel and electrophoresed in 1x NuPAGE MES SDS running buffer for 1.5 to $2 \mathrm{~h}$ at $160 \mathrm{~mA}, 160 \mathrm{~V}$ and $100 \mathrm{~W}$. The transfer of the proteins to a nitrocellulose membrane (GE Healthcare) was performed using a semi-dry blotting device at $120 \mathrm{~mA}, 20 \mathrm{~V}$ and $100 \mathrm{~W}$ for $90 \mathrm{~min}$. After blotting the membrane was blocked in $5 \%$ milk in TBST for $1.5 \mathrm{~h}$ at RT. The membrane was washed 3 times for $10 \mathrm{~min}$ in TBS with $0.1 \%$ Tween 20 and incubated overnight at $4{ }^{\circ} \mathrm{C}$ with the appropriate primary antibodies (see Table 14). After washing 3 times for $10 \mathrm{~min}$ in TBS with $0.1 \%$ Tween 20 membranes were 
incubated in HRP-conjugated secondary antibody for $1 \mathrm{~h}$ at RT. After another 3 washing steps 1-3 $\mathrm{ml}$ of the detection reagent (Amersham ECL Plus ${ }^{\mathrm{TM}}$ Western Blotting Detection Reagent) was added onto the membrane (so that the membrane was totally covered with the substrate) and incubated for $2 \mathrm{~min}$ at RT. For visualization of the proteins the FluorchemQ camera system and software was used.

\subsubsection{Ras activation ELISA}

For the detection of Ras activity in cell lines and tissue a commercially available ELISA kit, (Ras Activation ELISA ASSAY Kit) was used according to the manufacturer's instruction.

For protein isolation from cell lines, $1 \times 10^{6}$ cells were grown on a $10 \mathrm{~cm}$ dish for $24 \mathrm{~h}$. The culture media was removed and the cells were washed twice with ice-cooled PBS. The cells were lysed according to the Kit specification. For the lysis $200 \mu 1$ lysis buffer were used. . For analysis of tissue samples $20 \mu \mathrm{g}$ of the tissue were homogenized with a homogenizer in $300 \mu \mathrm{l}$ $\mathrm{Mg}^{2+}$ lysis/wash buffer in a $2 \mathrm{ml}$ tube and centrifuged at $14000 \mathrm{rpm}$ for $10 \mathrm{~min}$ at $4{ }^{\circ} \mathrm{C}$. The supernatant was transferred to a new $1.5 \mathrm{ml}$ tube and the protein concentration of the lysates was measured by Pierce ${ }^{\circledR}$ BCA Protein Assay Kit according to manufacturer's instructions.

Then the glutathione coated wells of the Kit were washed with TBST (washing buffer) and incubated for 1 with Raf-1-RBD, which is able to bind via a GST/Glutathione interaction to the coated wells. After a washing step the cell lysate $(50 \mu \mathrm{g})$ were added to the wells and incubated at RT for 1 the active/GTP-bound Ras proteins of the lysates are able to bind to the Ras binding domain of Raf-1 whereas the inactive/GDP-bound RAS will be washed away during the next washing step. After another washing step the wells are incubated with a monoclonal anti-Ras antibody specific for the detection of N-, K-, HRas isoforms, followed by another washing step. Next, the HRP conjugated secondary antibody was incubated for $1 \mathrm{~h}$. At the end the wells were washed with TBST followed by another washing step with TBS to remove the Tween 20 from the wells. For the detection the chemiluminescent substrate was added and the signals were measured by using a luminometer.

\subsubsection{Hematoxylin-Eosin $(H \& E)$ staining of paraffin embedded tissue}

For H\&E staining of paraffin embedded tissue, the tissue was cut into 4-5 $\mu \mathrm{m}$ sections, transferred to a slide and dried at $80{ }^{\circ} \mathrm{C}$ for $20 \mathrm{~min}$. Then the sections were deparaffinized 2 times for $10 \mathrm{~min}$ in Xylol and subsequently rehydrated by using descending ethanol solutions 
$(100 \%$ to $70 \%)$. After $2 \mathrm{~min}$ incubation in $\mathrm{ddH} 2 \mathrm{O}$ the sections were transferred to hematoxylin solution for $15 \mathrm{~min}$. For coloring the sections were incubated with flowing tepid tab water for $5 \mathrm{~min}$. Then the sections were shortly dipped into $1 \%$ eosin-solution with freshly added glacial acetic acid $(0.5 \% \mathrm{v} / \mathrm{v})$ and washed with ddH2O. At the end the sections were dehydrated by using ascending ethanol solutions (70\% to $100 \%$ ethanol), shortly placed in xylene and mounted in Pertex.

\subsubsection{Immunohistochemistry}

For immunohistochemistry staining, paraffin embedded tissue was cut at $2-4 \mu \mathrm{m}$ sections, deparaffinized by xylene and rehydrated using descending ethanol solutions (100\%, $95 \%$ to $35 \%$ ). If necessary, a permeabilization treatment was done as described in Table 14. Next, the sections were blocked with $3 \% \mathrm{H} 2 \mathrm{O} 2$ to inhibit endogenous peroxidase. This was followed by a blocking step with $0.2 \%$ casein to prevent non-specific antibody binding. Next, the sections were incubated with a primary antibody (see Table) for $1 \mathrm{~h}$ at RT in a humid chamber. After washing with TBS the sections were incubated with the appropriate secondary antibody for $1 \mathrm{~h}$ at RT. After another wash step the visualization of the antibody binding was done either by using DAB+ (Envision+ system-HRP, Dako) as chromogen. At the end the reaction was stopped by transferring the sections to distilled water. Counterstaining was performed with hematoxilin and the slides were embedded in Glycergel ${ }^{\circledR}$ Mounting Medium (Dako, Hamburg).

The quantification of Ki-67 positive cells was performed using the imageJ software. For this purpose 5 representative pictures per section were taken and 1000 cells of these pictures were counted. The Ki-67 positive cells are given in percent.

\subsection{7. $\quad$ X-Gal staining of cryo-embedded tissue}

For X-Gal staining the tissue was fixed for $2 \mathrm{~h}$ with $4 \%$ paraformaldehyde/PBS on ice, thoroughly washed and incubated in $25 \%$ sucrose/PBS overnight. The next day, the tissue was embedded in OCT cryoblock embedding media on dry ice and cut into $5 \mu \mathrm{m}$ sections at -20 to $-22^{\circ} \mathrm{C}$ and mounted onto slides and dried at RT for $2 \mathrm{~h}$. Then the sections were incubated for 10 min with $0.2 \%$ Glutaraldehyd /PBS on ice. After incubation in LacZ-buffer 3 times for 10 min the sections were incubated overnight in LacZ-staining solution (ingredients see Table X) 
at $30{ }^{\circ} \mathrm{C}$. The next day the sections were mounted with Glycergel ${ }^{\circledR}$ Mounting Medium (Dako, Hamburg).

\subsection{Animal Experiments}

All animal experiments described in this thesis were performed in compliance with all relevant legal and ethical requirements.

\subsubsection{Breeding and keeping of mice}

All mouse lines used in this thesis were bred and kept in the private institute animal facility of the Institute of Human Genetic, University of Göttingen. The Ptch ${ }^{d e l /+}, L S L-K-R A S G 12 D^{f l /+}$, NRAS LSL-G12D $D^{\mathrm{fl} /+}, F R-H R A S G 12 V^{f l /+}$, Myf5creERT $T^{t g /+}$ and ROSA26-R ${ }^{f l /+}$ mice were housed in Makrolon cages type I and II and the immune deficient NMRI-Foxn $1^{n u}$ mice were housed in specific pathogen free (SPF) cages. All animals were housed in rooms with a twelve-hour light-dark cycle (light period: 6.a.m.-6 p.m.), a temperature of $20 \pm 2^{\circ} \mathrm{C}$ and a relative humidity of $50 \pm 10 \%$. They were fed ad libitum with rodent pellets (complete diet for mice breeding) and tap water which was autoclaved in case of the NMRI-Foxn $1^{n u}$ mice.

\subsubsection{Tail biopsy and genotyping of mice}

For identification of the mice the weaned mice (age approx. 4 weeks) were labeled by earclipping. Respective tail biopsies (approx. a peace of $0.2 \mathrm{~cm}$ from the tip of the tail) were taken at the age of four weeks. Mice that were younger and not yet weaned were labeled by a tattoo code on their foot pads and later at the age of four weeks by an ear code.

DNA isolation from tail biopsies and genotyping was performed by PCR. The used oligonucleotides and PCR conditions are listed in Table 11.

\subsubsection{Tamoxifen treatment of mice}

To induce the expression of mutant KRAS, HRAS or NRAS in Ptch $^{\text {del/ }}$ oncRAS $S^{f l /-}$ Myf5CreERT ${ }^{\text {tg/- }}$ mice (see chapter 4.9.3) the animals were injected with tamoxifen (SigmaAldrich, Chemie $\mathrm{GmbH}$, Steinheim). For preparing a $10 \mathrm{mg} / \mathrm{ml}$ tamoxifen solution, $200 \mathrm{mg}$ tamoxifen was emulsified in $800 \mu 199.9 \%$ EtOH and diluted with sunflower oil (SigmaAldrich, Chemie $\mathrm{GmbH}$, Steinheim) to a $20 \mathrm{ml}$ final volume. The tamoxifen solution was stored until application at $-20{ }^{\circ} \mathrm{C}$. For the activation of the Cre recombinase $100 \mu 1$ of the 
tamoxifen solution (dosis of $1 \mathrm{mg}$ tamoxifen) was injected intraperitoneally (i.p.) for five consecutive days (final dose of $5 \mathrm{mg}$ tamoxifen) starting at an age of 4 weeks.

\subsubsection{Tumor monitoring and isolation}

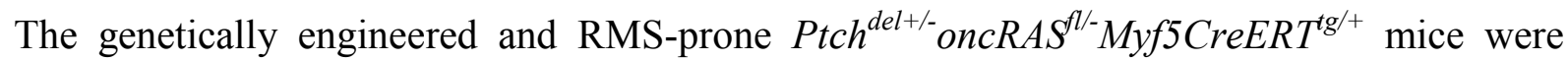
monitored each week. Soft tissue tumors of the animals were detected by manual palpation. In addition, they were checked for symptoms of medulloblastoma namely ataxia, emaciation, lethargy or development of a hydrocephalus. Animals which showed this type of symptoms were painlessly killed by $\mathrm{CO}_{2}$ anesthesia and cervical dislocation. When soft tissue tumors were detected, the date was documented. The animals were also sacrificed when the tumor reached $1 \mathrm{~cm}$ in size or after the monitoring time (200 days after birth). All mice were autopsied and all parable and non-palpable tumors and skeletal muscle as reference tissue were isolated and fixed in $4 \%$ PFA-solution overnight.

\subsubsection{Anesthesia of mice}

An anesthesia-solution was prepared containing $1 \mathrm{ml}$ Ketamin Inresa $(50 \mathrm{mg} / \mathrm{ml}$ Ketaminhydrochlorid) and $400 \mu \mathrm{l}$ Xylazin (2\% Xylazinhydrochlorid) dissolved in $1.4 \mathrm{ml}$ $\mathrm{ddH}_{2} \mathrm{O}$. The solution was injected intraperitoneally at a concentration of $3.6 \mu 1 / \mathrm{ml}$ body weight. For preventing drying of the eyes during anesthesia the eyes were covered with Bepanthen ${ }^{\circledR}$ eye and nose lotion (Bayer Vital GmbH).

\subsubsection{Tumor xenograft}

For transplantation of RMS cells in nude mice the cells were grown until they were $80-90 \%$ confluent. Media was discarded and the cells were trypsinized and pelleted at $300 \mathrm{~g}$ for $5 \mathrm{~min}$ at $4{ }^{\circ} \mathrm{C}$. Next, the cells were washed two times with PBS and dissolved in 500-1000 $\mu$ PBS. The amount of viable cells was determined by counting trypan blue stained cells in a Neubauer counting chamber. The RMS cells were transferred to pre-chilled syringes in a total volume of $100 \mu \mathrm{l}$ containing $5 \times 10^{6}$ (RMS-13) cells per mouse. The cells were transplanted into the flanks of anesthetized nude mice at the age of $8-10$ weeks. The mice were controlled daily and the tumor size was measured using a digital caliper. The calculation of the tumor volume was done according to the following formula (Tomayko and Reynolds 1989). 


$$
V=\frac{(\text { Length } \times \text { Broad } \times \text { High })}{2}
$$

All mice were sacrificed at the end of the experiment at approximately 30 days after transplantation and the tumors were isolated, fixed in $4 \%$ paraformaldehyde and embedded in paraffin for histological analysis. If the tumor was large a part of the tumor was stored at $80^{\circ} \mathrm{C}$ for further analysis. 


\section{Results}

\subsection{Effects of oncogenic $\mathrm{N}$-, K- or HRAS (in short oncRAS) on human RMS cell lines}

\subsubsection{Generation of oncRAS RMS cell lines}

To investigate whether oncogenic N-, K- or HRAS (in short oncRAS) can influence HH signaling in human RMS cells, pMSCVpuro plasmids containing inserts of constitutive active forms of RAS (pMSCVpuro-NRAS12V, pMSCVpuro-KRAS12V or pMSCVpuro-HRAS12V) were generated (see chapter 4.15.9). After sequencing, the plasmids were stably transduced in RMS cell lines that were wildtype RAS (wtRAS), namely RMS-13 (ARMS) and RUCH-2 (ERMS) cells (see chapter 4.14.2) (Schaaf et al. 2010). As control, the cell lines were transduced with the empty pMSCVpuro vector.

\subsubsection{Successful transduction of oncRas in RMS cell lines}

To confirm the successful transduction with pMSCVpuro, pMSCVpuro-NRAS12V, pMSCVpuro-KRAS12V or pMSCVpuro-HRAS12V, cDNA of the cells was prepared. After amplification with pMSCVpuroF (that binds to vector-derived sequences of the cDNA) and oncRAS-specific reverse primers (i.e. pNRasID-Rev, pKRasID-Rev or pHRasID-Rev) the respective PCR-fragments (Figure 4; shows data for RUCH-2 cells as an example) were also sequenced. Indeed, all oncRAS plasmids expressed the respective oncogenic versions of the RAS genes in exon 2 (Figure 5; shows data for RUCH-2 cells as an example).

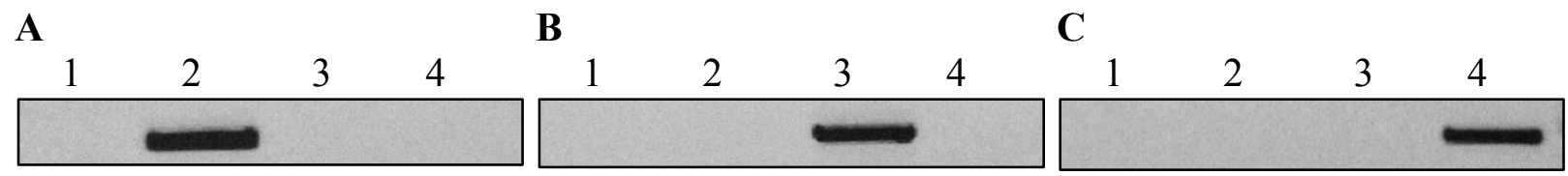

Figure 4: PCR Fragments amplified with pMSCV-seq-F and either pNRasID-Rev, pKRasID-Rev or pHRasID-Rev from cDNA isolated from RUCH-2 cells. cDNA isolated from RUCH-2 cells transduced with pMSCV (Line 1), RUCH-2 NRAS12V (Line 2), RUCH-2 KRAS12V (Line 3) and RUCH-2 HRAS12V(Line 4). PCR with the primers (A) pMSCV-seq-F/pNRasID-Rev (B) pMSCV-seq-F/ pKRasIDRev (C)pMSCV-seq-F/ pHRasID-Rev. 


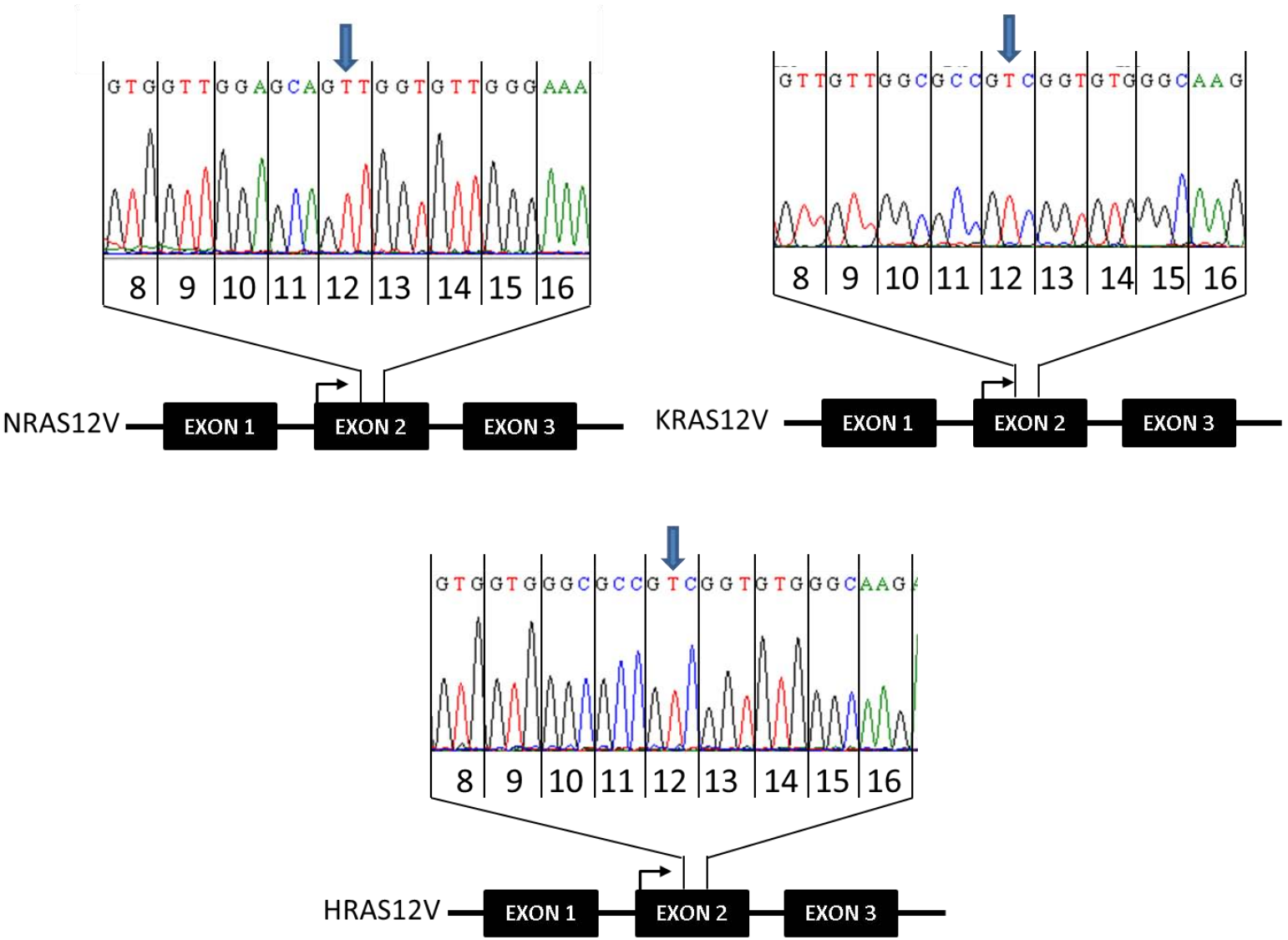

Figure 5: Sequences of the PCR fragments shown in Figure 4. Shown are the sequences of the second exons of oncogenic N-, K- and HRAS. The codon 12 located in exon 2 encodes for valine (instead of glycine), which results in constitutive activation of the corresponding RAS protein.

\subsubsection{Increased RAS expression levels in oncRas transduced RMS cell lines}

In order to investigate, if the stable transduction of the oncRAS plasmids in RMS cell lines resulted in elevated levels of RAS protein, a Western Blot analysis was performed using an anti-Pan-RAS antibody (see Figure 6). 
A

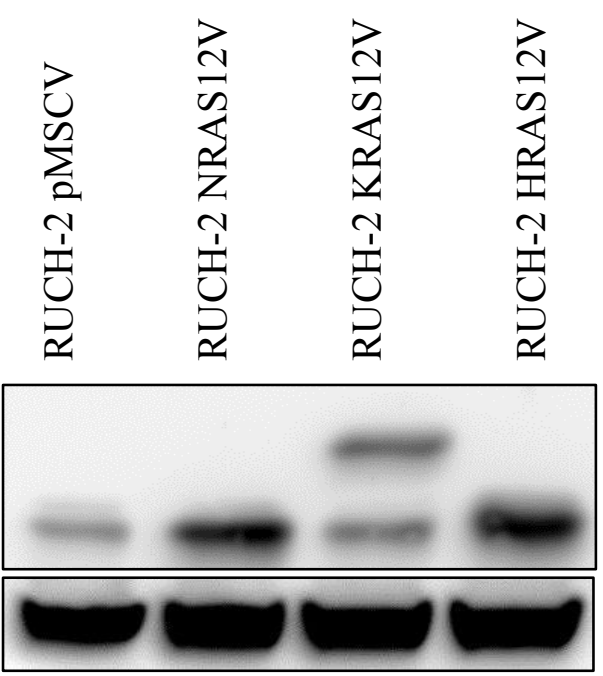

B

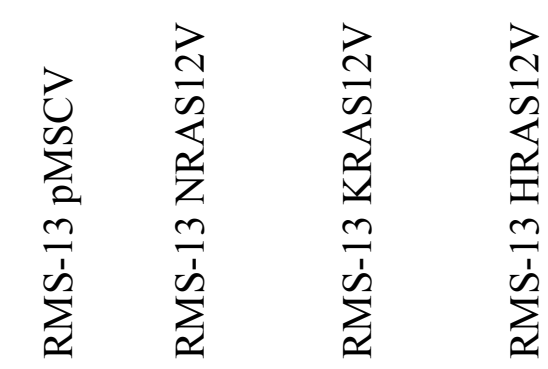

Pan-RAS

$[21 \mathrm{kDa}]$

HSC70

$[70 \mathrm{kDa}]$

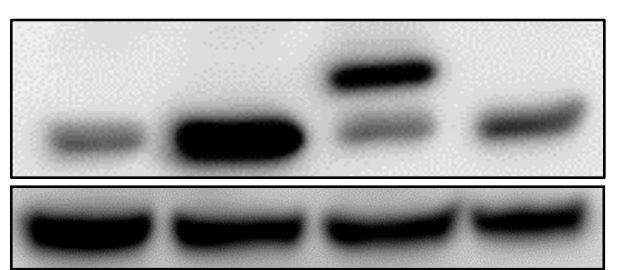

Figure 6: Elevated RAS levels in RMS cell lines transduced with oncRAS. Western blot analyses using anti-Pan-RAS antibody with protein lysates of (A) RUCH-2 (ERMS) and (B) RMS-13 (ARMS) cells stably transduced with pMSCVpuro (pMSCV), pMSCVpuro-NRAS12V (NRAS12V), pMSCVpuro-KRAS12V (KRAS12V), or pMSCVpuro-HRAS12V (HRAS12V). Detection of the HSC70 expression levels served as loading control.

As illustrated in Figure 6 protein samples collected from pMSCVpuro transduced RMS cells (RUCH-2 pMSCVpuro and RMS-13 pMSCVpuro) express low levels of RAS, as shown by a weak band at the expected size of $21 \mathrm{kDa}$. The cells expressing oncogenic RAS show moderately increased RAS expression levels. In cells that had been transduced with pMSCVPuro-KRAS12V a double band can be detected. The larger protein is the mutant KRAS12V since KRAS12V expressed by the plasmid pMSCVpuro-KRAS12V is HA-Tagged (see Figure Appendix 1, 2 and 3).

\subsubsection{No obvious morphological changes of oncRAS transduced RMS cell lines}

Yeh et al. showed that the activation of the RAS signaling pathway can change the morphology of different cell lines (Yeh et al. 2008). However, as illustrated in Figure 7, none of the cell lines stably transduced with oncRAS showed distinct morphological changes compared to control vector transduced cells. 

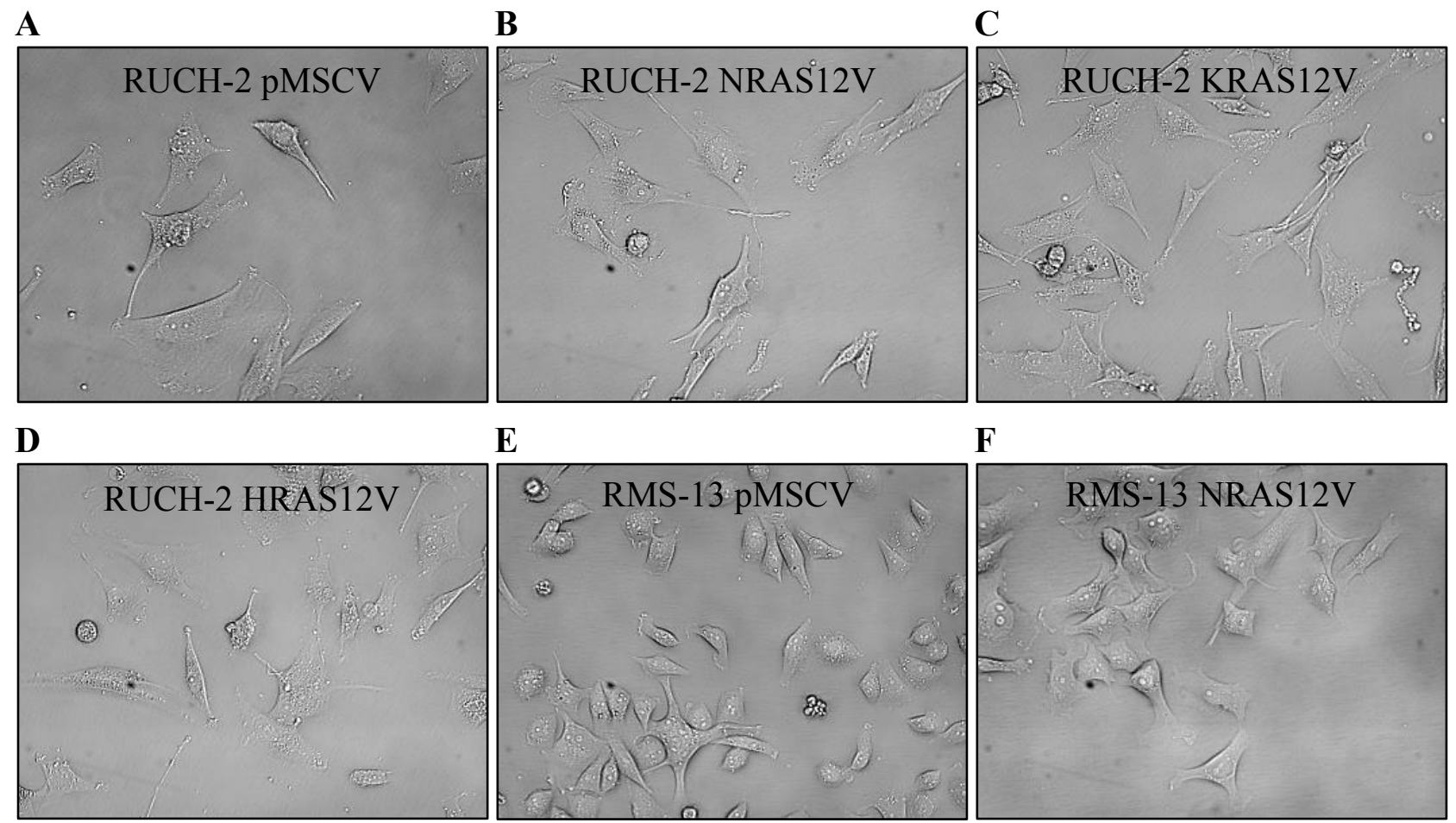

E

$\mathbf{F}$
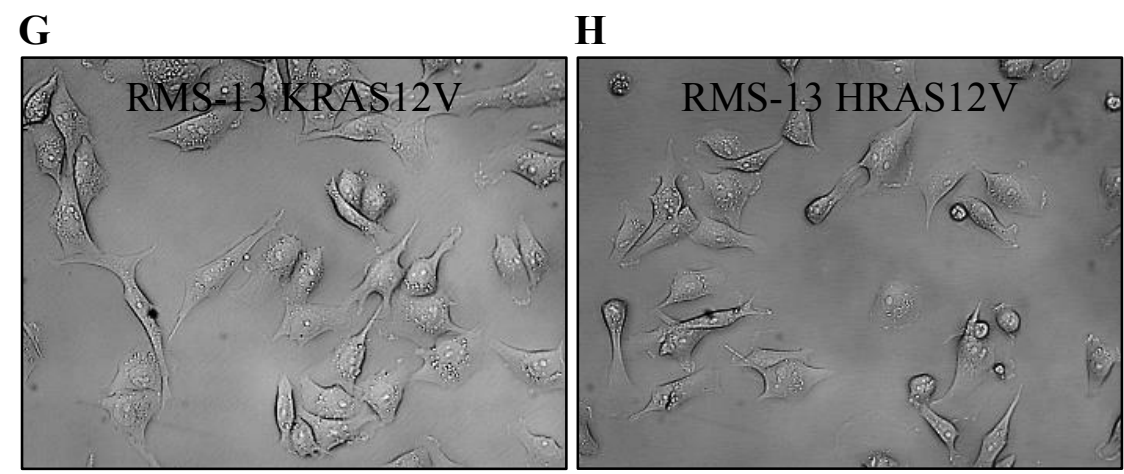

Figure 7: Morphology of oncRAS transduced RMS cell lines. Shown are representative pictures of RUCH-2 (A-D) and RMS-13 (E-H) cells stably transduced with pMSCVpuro (pMSCV), pMSCVpuroNRAS12V (NRAS12V), pMSCVpuro-KRAS12V (KRAS12V), or pMSCVpuro-HRAS12V (HRAS12V) as indicated (20 x magnification).

\subsubsection{Inhibition of $G L I 1$ expression in oncRAS transduced RMS cell lines}

To see whether active RAS signaling can modulate $\mathrm{HH}$ signaling the expression levels of GLI1 in the RAS transduced cell lines were measured. As mentioned in the introduction the expression level of GLII is a reliable marker for $\mathrm{HH}$ signaling activity (Hooper and Scott 2005). As shown in Figure 8 overexpression of all 3 oncogenic RAS isoforms decreased GLII expression levels as revealed by qRT-PCR. This indicates that oncRAS probably inhibits the HH-signaling pathway in both ERMS (RUCH-2) and ARMS (RMS-13) cells. This is similar 
to data shown by Lauth et al. (Lauth et al. 2010), who demonstrated that KRAS12V inhibits HH signaling and thus GLI1 expression by blocking GLI2/3 (see introduction).

A

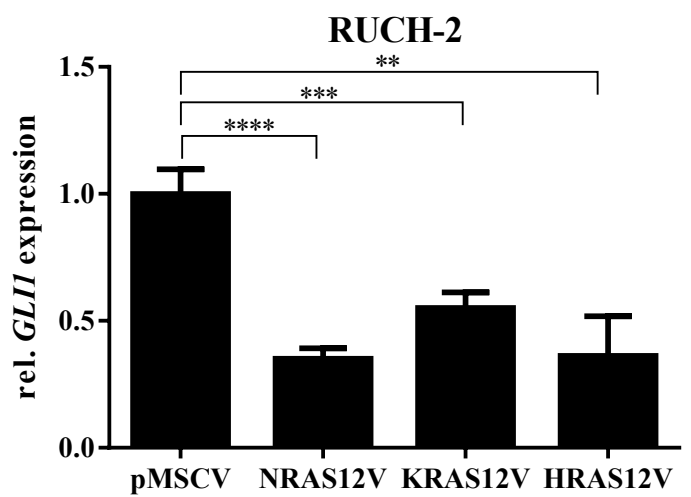

B

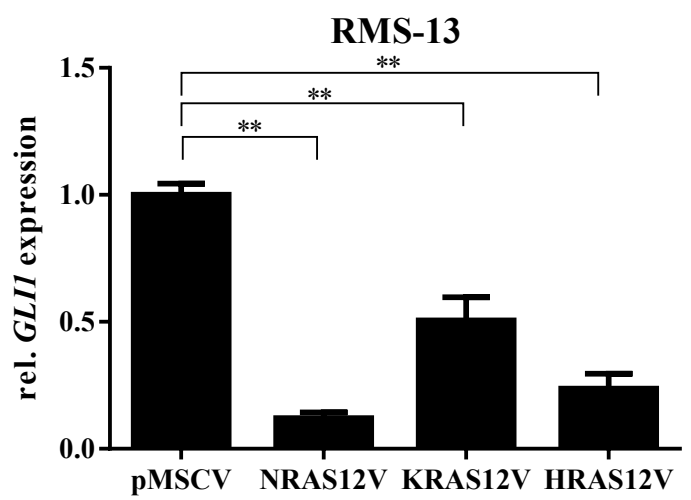

Figure 8: Downregulation of GLII expression in oncRAS transduced RMS cell lines. Quantification of GLI1 expression levels by qRT-PCR in (A) RUCH-2 (ERMS) and (B) RMS-13 (ARMS) cells stably transduced with the empty vector pMSCVpuro (pMSCV), pMSCVpuro-NRAS12V (NRAS12V), pMSCVpuro-KRAS12V (KRAS12V) or pMSCVpuro-HRAS12V (HRAS12V), as indicated. Expression levels were normalized to $18 S$ rRNA levels. GLI1 expression of the pMSCVpuro-transduced cells was set to 1 . All data represent at least 3 independent experiments measured in triplicates displayed as mean \pm SEM. Statistical significance was tested by using Mann-Whitney test. ${ }^{*} \mathrm{P}<0.05$, ${ }^{* *} \mathrm{P}<0.005,{ }^{* * *} \mathrm{P}<0.0005$ and $* * * * \mathrm{P}<0.0001$.

Since KRAS12V simultaneously can induce SHH in pancreatic cancer cells (Lauth et al. 2010), SHH expression levels were analyzed by qRT-PCR. The data show that oncRAS indeed induced $S H H$ expression in RUCH-2 cells. However, this was not the case in RMS-13 cells, in which oncRAS inhibited $\mathrm{SHH}$ expression (Figure 9).

A

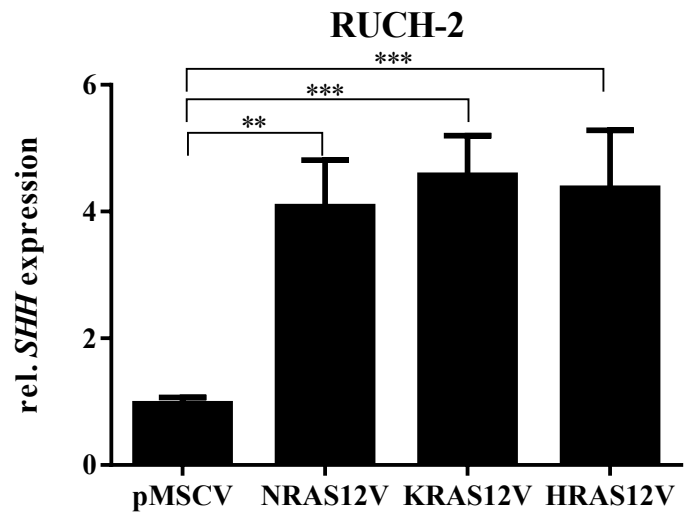

B

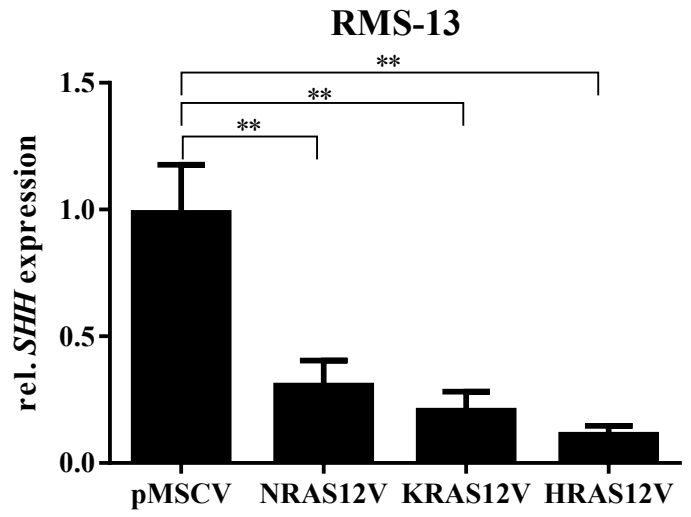

Figure 9: $S H H$ expression levels in oncRAS transduced RMS cell lines. Quantification of $S H H$ expression levels by qRT-PCR in (A) RUCH-2 and (B) RMS-13 cells stably transduced with the empty vector pMSCVpuro (pMSCV), pMSCVpuro-NRAS12V (NRAS12V), pMSCVpuro-KRAS12V (KRAS12V) or pMSCVpuro-HRAS12V (HRAS12V). Expression levels were normalized to $18 S$ rRNA levels. SHH 
expression of the pMSCVpuro-transduced cells was set to 1 . All data represent at least 2 independent experiments measured in triplicates displayed as mean \pm SEM. Statistical significance was tested by using Mann-Whitney test. * $\mathrm{P}<0.05, * * \mathrm{P}<0.005$ and $* * * \mathrm{P}<0.0005$.

Next, it was analyzed whether the transduced RUCH-2 cells were able to secrete SHH. For this purpose the supernatant of the cells was harvested as described in chapter 4.14.3. The murine HH-responsive fibroblast cell line B9 (also called Ptch ${ }^{\text {floxflox } C r e E R T 2 ~ c e l l s ;(U h m a n n ~}$ et al. 2011)) was incubated with the respective supernatants and the Glil expression was measured by qRT-PCR. Supernatant from HEK293-SHH cells served as control. However, as seen in Figure 10, RUCH-2 cells transduced with oncRAS were not able to secrete SHH.

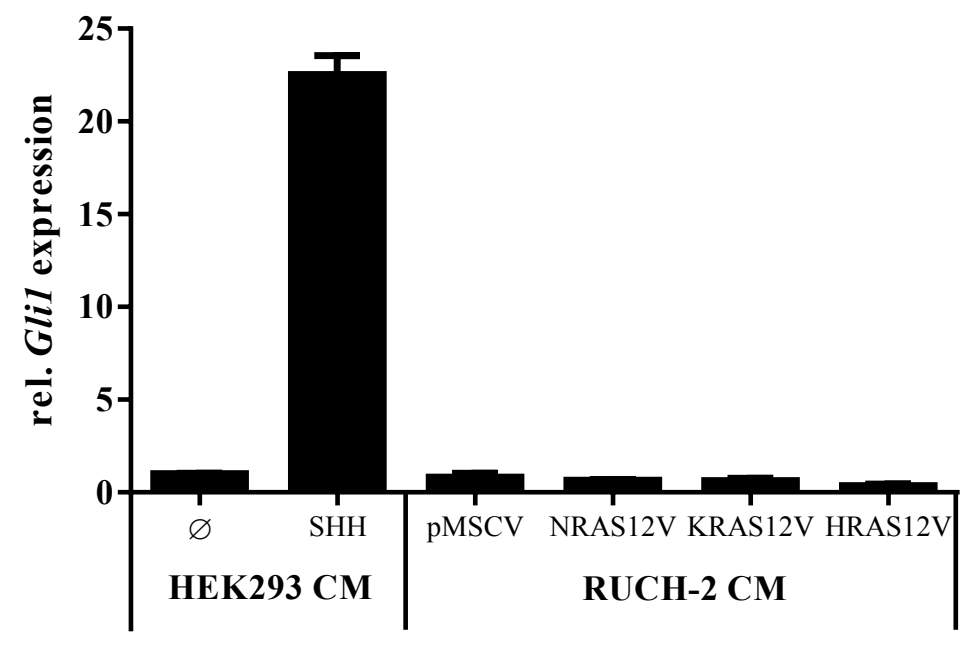

Figure 10: oncRAS transduced RUCH-2 cell lines are not able to secrete SHH. Quantification of Gli1 expression levels by qRT-PCR analyses in B9 cells incubated with conditioned medium (in short CM) from HEK293-wildtype $(\varnothing)$ and HEK293-SHH (SHH) cells and from RUCH-2 cells stably transduced with the empty vector pMSCVpuro (pMSCV), pMSCVpuro-NRAS12V (NRAS12V), pMSCVpuro-KRAS12V (KRAS12V) or pMSCVpuro-HRAS12V (HRAS12V). Expression levels were normalized to $18 S$ rRNA levels. Gli1 expression of B9 cells treated with the HEK293 wildtype-CM was set to 1. All data represent 1 experiment measured in triplicates displayed as mean \pm SEM. Statistical significance was tested by using Mann-Whitney test.

Together these data show that oncRAS suppresses GLII expression in both RUCH-2 and RMS-13 cell lines. In addition, oncRAS induced SHH expression, but not secretion, in RUCH-2 cells, whereas SHH expression was repressed in RMS-13 cells. 


\subsubsection{Inhibition of GLI1 expression in oncRAS transduced RUCH-2 depends on MEK activity}

In order to investigate if oncRAS inhibited $\mathrm{HH}$ signaling via the RAF/MEK/ERK or $\mathrm{PI} 3 \mathrm{~K} / \mathrm{AKT} / \mathrm{mTOR}$ axis cells were treated with the MEK inhibitor UO126 or the dual PI3K/mTOR inhibitor PI103. In addition, the cells were incubated with HhAantag (HhA) that binds and inhibits SMO and thus can block canonical HH signaling (Figure 11). However, as recently shown by our group HhA can additionally reduce phosphorylation of AKT in ERMS cell lines (Dissertation Rosalie Ridzewski). GLII expression served as readout.

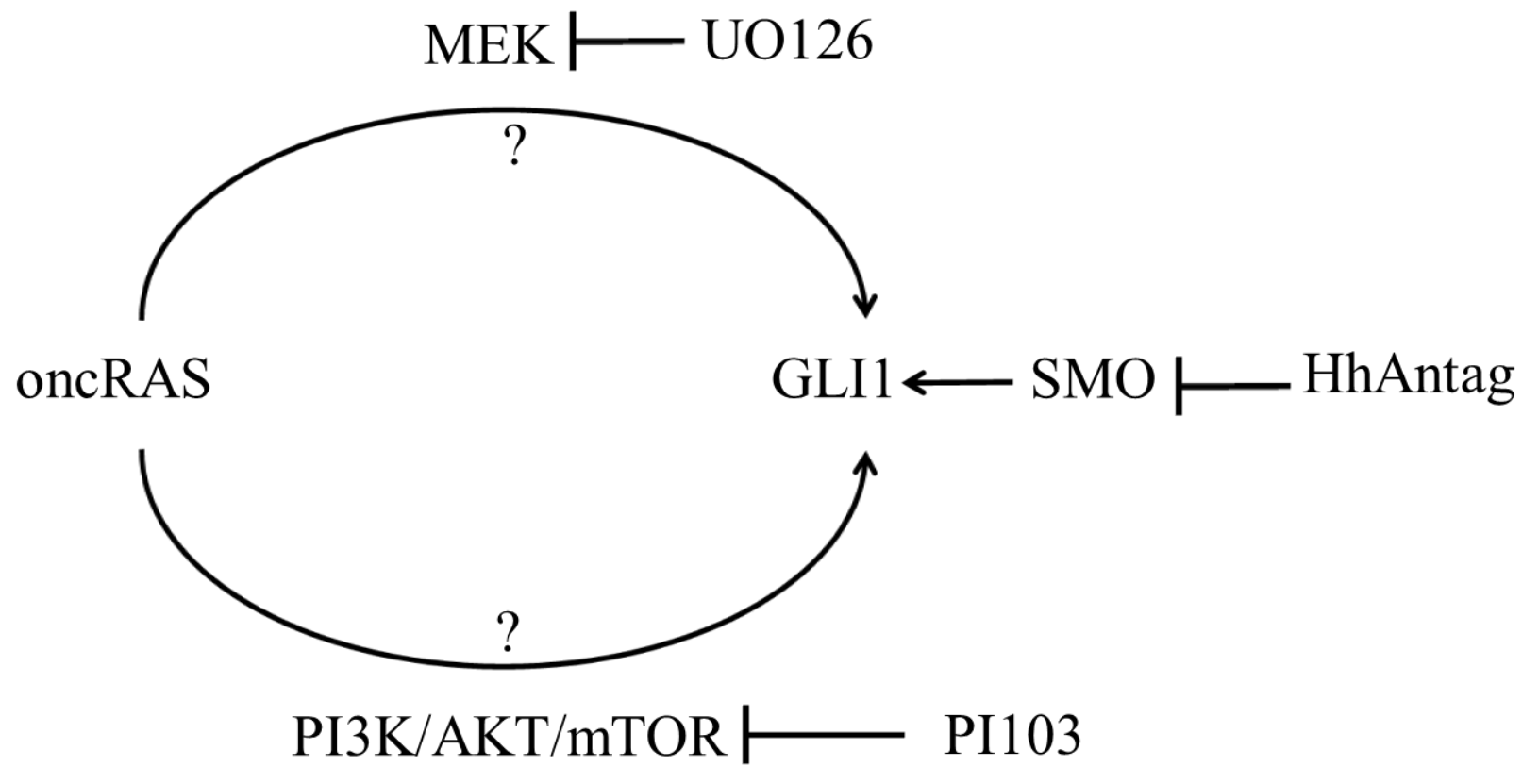

Figure 11: Schematic representation: Treatment strategy of stably transduced RMS cells. As shown in Figure 8 oncRAS inhibits GLI1 expression. In order to see whether this is mediated by the RAF/MEK/ERK axis the MEK inhibitor UO126 that is a dual MEK1 \& MEK2 inhibitor and therefore also blocks the phosphorylation of the downstream targets ERK1 and ERK2 (Favata et al. 1998) was employed. The dual PI3K/mTOR inhibitor PI103 which can inhibit both the rapamycin-sensitive and -insensitive complexes of mTOR and block PI3K activity and thus also its downstream effector AKT (Fan et al. 2006, Knight et al. 2006), was used to inhibit the PI3K/AKT/mTOR axis. The SMO inhibitor HhA that supposedly blocks canonical HH signaling (Dijkgraaf et al. 2011) and can show interaction with AKT activity was used as a control.

As shown in Figure $12 \mathrm{HhA}$ and PI103, but not UO126, inhibited GLI1 expression and thus HH signaling activity in RUCH-2 (Figure 12A) and RMS-13 (Figure 12E) control cells. In RUCH-2 cells that express oncRAS, UO126, but not PI103 or HhA, increased HH signaling activity back to normal levels (Figure 12B-D; values not significant in comparison to the 
solvent-treated control cell line). This suggested that oncRAS-induced downregulation of GLI1 expression in RUCH-2 cells depends on MEK activity. In contrast in RMS-13 that express oncRAS (Figure 12F-H) and show decreased expression of GLII when compared to the control, the inhibitors did not result in any changes in GLII expression.

A

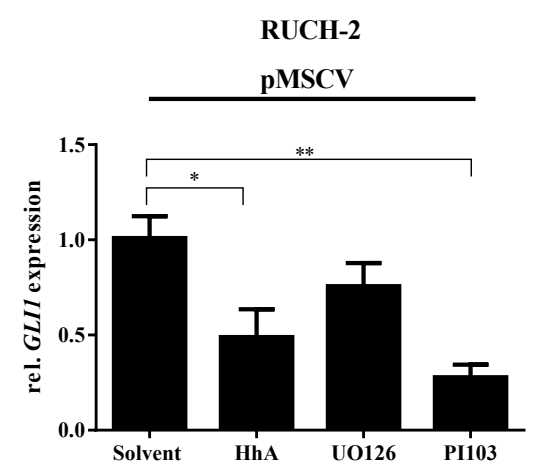

D

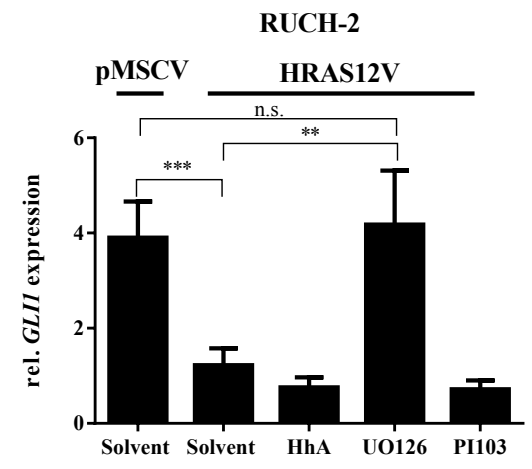

G

RMS-13

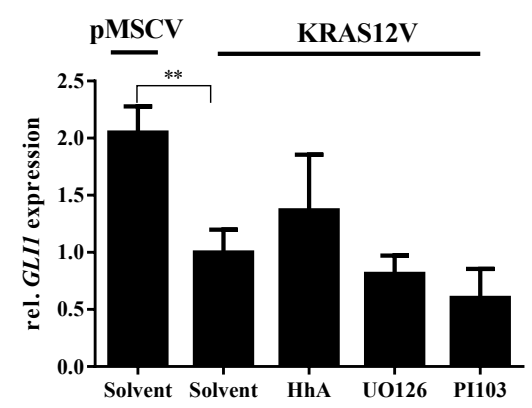

B

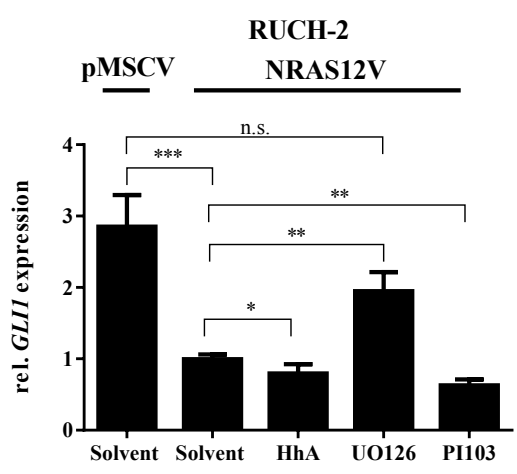

$\mathbf{E}$

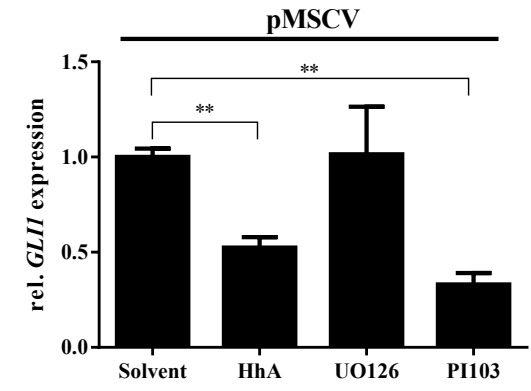

$\mathbf{H}$

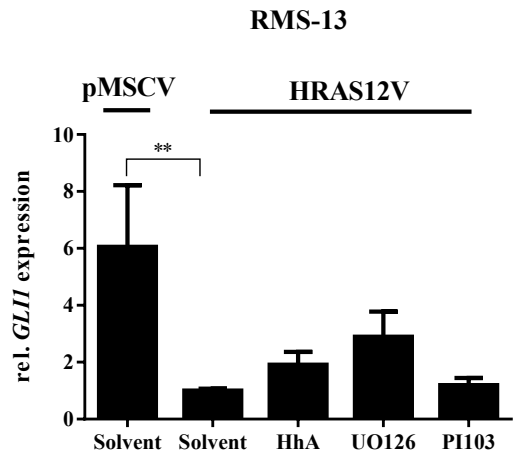

C

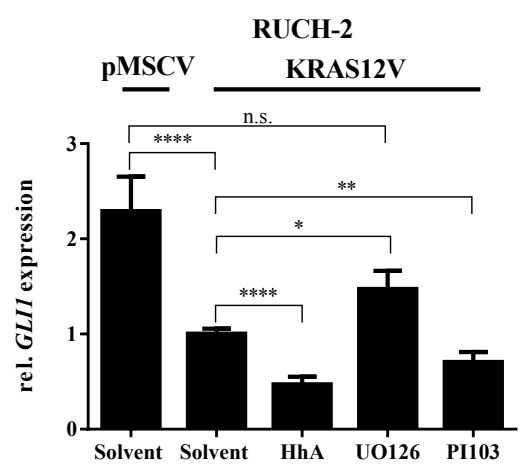

$\mathbf{F}$

RMS-13

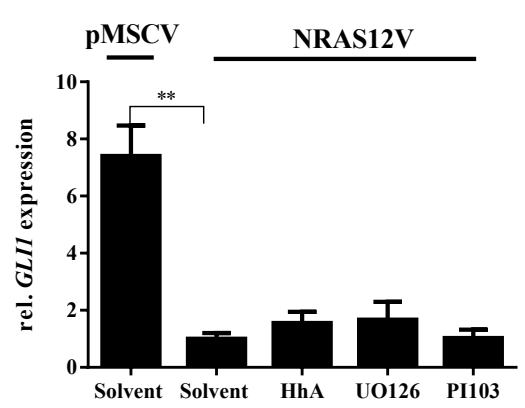

Figure 12: oncRAS may inhibit GLI1 expression via the MEK/ERK axis in RUCH-2, but not RMS-13 cells. Quantification of GLII expression levels measured by qRT-PCR analyses of (A-D) RUCH-2 (ERMS) and (E-F) RMS-13 (ARMS) cells stably transduced with pMSCVpuro (pMSCV), pMSCVpuro-NRAS12V (NRAS12V), pMSCVpuro-KRAS12V (KRAS12V) or pMSCVpuro-HRAS12V (HRAS12V), as indicated. Cells were treated for $24 \mathrm{~h}$ with solvent, HhA (SMO inhibitor), UO126 (MEK inhibitor) or PI103 (Dual PI3K/mTOR inhibitor). Expression levels were normalized to $18 S$ rRNA levels. GLII expression levels of 
the solvent controls were set to 1 (please note that in the figures showing data of solvent treated control and oncRAS cell lines, the data of solvent treated oncRAS cell lines were set to one) All data represent at least 3 independent experiments measured in triplicates displayed as mean \pm SEM. Statistical significance was tested by using Mann-Whitney test. * $\mathrm{P}<0.05$, ** $\mathrm{P}<0.005$, *** $\mathrm{P}<0.0005$ and $* * * \mathrm{P}<0.0001$. Please note that in the Figures showing the comparison of control and oncRAS transduced cells, significant values are only shown for the comparison of the solvent-treated cells and not for the comparison of the solvent-treated control and oncRAS transduced cells that have been treated with the inhibitors. The only exception is the comparison of solvent-treated control RUCH-2 cells with oncRAS-transduced cells that have been treated with UO126.

Together the data show that oncRAS mutations in both ERMS and ARMS cell lines decrease GLII expression, which is indicative for blockage of the HH signal cascade. However, the mode of action seems to be different. Whereas in the ERMS cell line RUCH-2 oncRASmediated inhibition of $\mathrm{HH}$ signaling is probably communicated via the MEK axis, the RASinduced inhibition of HH signaling in the ARMS cell line RMS-13 is not.

\subsection{7. oncRAS increase pERK levels in RMS-13 and RAS activity in RUCH-2}

Next, the phosphorylation status of ERK and AKT in oncRAS-transduced cell lines was examined. For this purpose protein lysates from stably transduced RUCH-2 and RMS-13 cells were used for Western blot analysis (see Chapter 4.16.3). Here, only unambiguous results of at least 3 Western Blots are discussed. As illustrated in Figure 13 and Figure 14 the expression of NRAS12V, KRAS12V, or HRAS12V increased phosphorylation of ERK in RMS-13 (Figure 14) but not in RUCH-2 cells (Figure 13) when compared to the respective cell line that have been transduced with the control plasmid pMSCVpuro.

The increased phosphorylation of ERK in RMS-13 was an indication that the expressed oncRAS proteins were functional. The lack of any changes in ERK and AKT phosphorylation in RUCH-2 prompted us perform a RAS Activation ELISA. As demonstrated in Figure 15, RAS signaling activity was increased in RUCH-2 cells transduced with oncRAS compared to the control cells. These data show that the expressed oncRAS proteins in RUCH-2 were functional as well. 


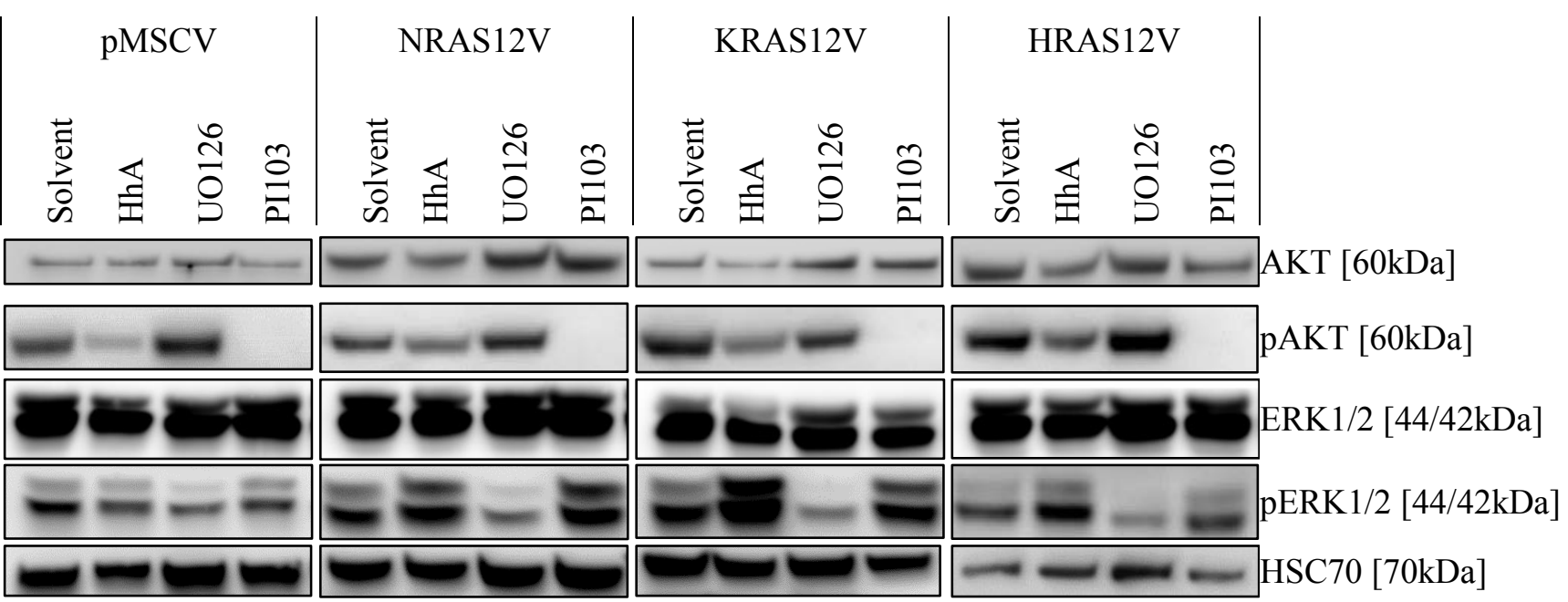

Figure 13: Modulation of RAS effector pathways in oncRAS-transduced RUCH-2 cells. Western blot analyses using antibodies specific for downstream targets of RAS signaling with protein lysates of RUCH2 cells stably transduced with pMSCVpuro (pMSCV), pMSCVpuro-NRAS12V (NRAS12V), pMSCVpuroKRAS12V (KRAS12V) or pMSCVpuro-HRAS12V (HRAS12V), as indicated. Cells were treated with solvent, HhA, UO126 or PI103 for 24h as indicated. Detection of the HSC70 expression levels served as loading control.

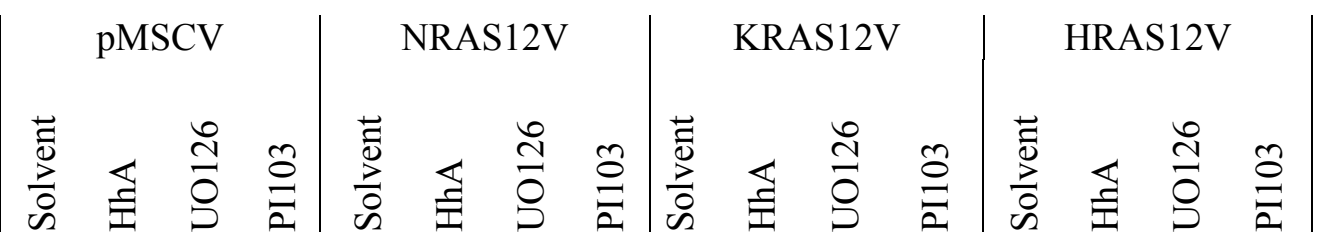

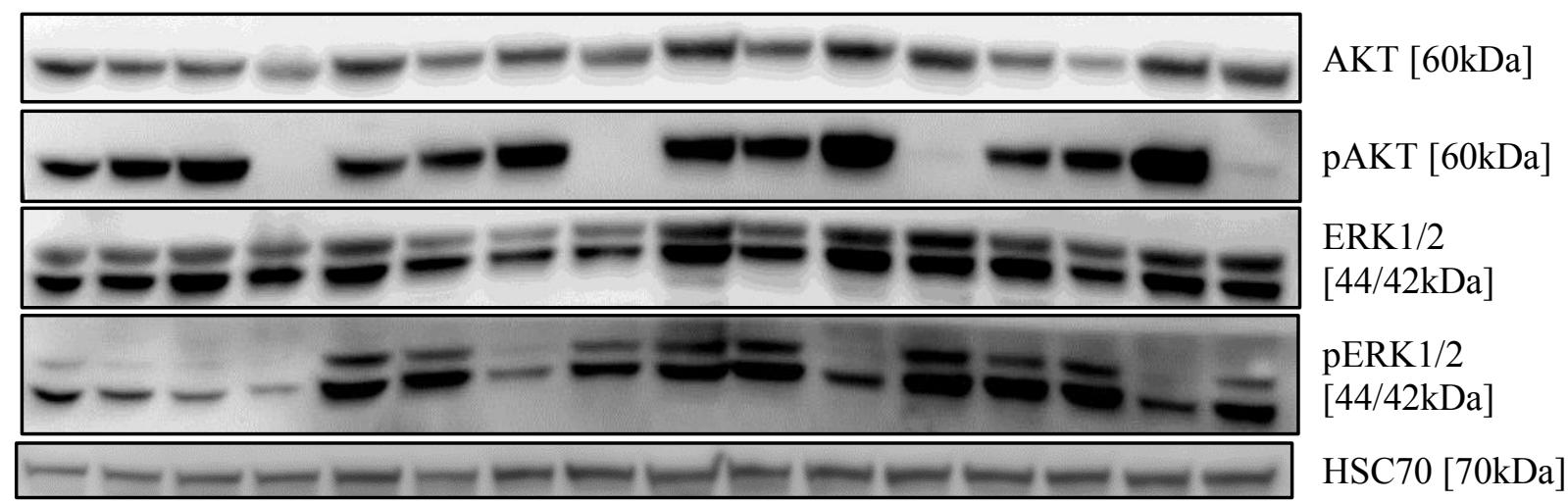

Figure 14: Modulation of RAS effector pathways in oncRAS-transduced RMS-13 cells. Western blot analyses using antibodies specific for downstream targets of RAS signaling with protein lysates of RMS-13 cells stably transduced with pMSCVpuro (pMSCV), pMSCVpuro-NRAS12V (NRAS12V), pMSCVpuroKRAS12V (KRAS12V) or pMSCVpuro-HRAS12V (HRAS12V), as indicated, and were treated with solvent, HhA, UO126 or PI103 for 24h as indicated. Detection of the HSC70 expression levels served as loading control. 


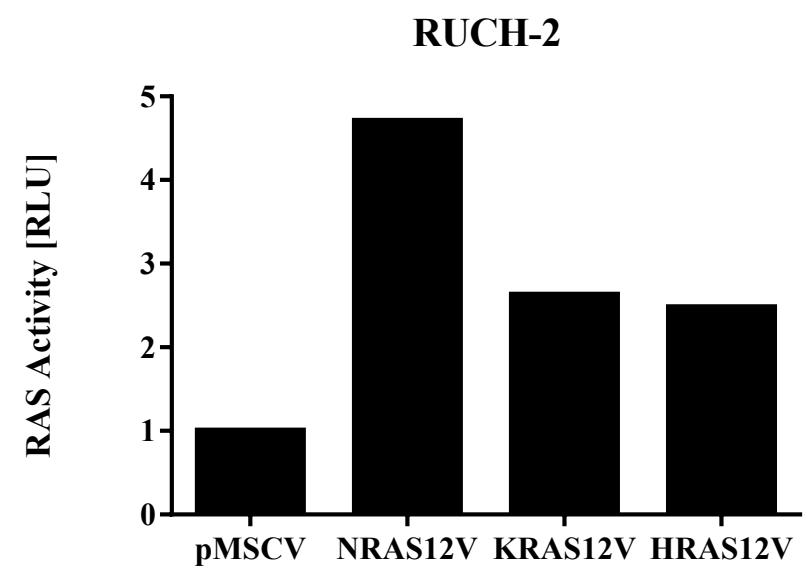

Figure 15: Moderately increased RAS activity in RUCH-2 cells stably transduced with oncRAS. RAS activity analysis using the Ras Activation ELISA ASSAY Kit in protein lysates of RUCH-2 cells stably transduced with either pMSCVpuro (pMSCV), pMSCVpuro-NRAS12V (NRAS12V), pMSCVpuroKRAS12V (KRAS12V) or pMSCVpuro-HRAS12V (HRAS12V), as indicated.

5.1.8. HhA decreases and increases pAKT and pERK levels, respectively in RUCH-2 cells, whereas UO126 decreases pERK and concomitantly induces pAKT levels in RMS 13 cells

In RUCH-2 cells, treatment with HhA decreased AKT/pAKT levels. In addition, it increased phosphorylation of ERK in RUCH-2 cells that express oncRAS (Figure 13 and Figure 14). As expected, treatment with UO126 decreased phosphorylation of ERK in all settings. Finally and equally expected, treatment of the cells with PI103 blocked phosphorylation of AKT.

In RMS-13 cells treatment with HhA did not affect any of the analyzed proteins, whereas treatment with UO126 decreased pERK and concomitantly induced pAKT levels, which also has been shown in RMS and other cell lines (Chen et al. 2012, Graham et al. 2006, Guenther et al. 2013). As expected, PI103 decreased pAKT levels. Otherwise it had no effects.

With respect to the decrease of GLII expression by oncRAS in RUCH2 and RMS-13 cells (see Figure 8) these data suggest that neither the phosphorylation status of AKT nor of ERK play a role in $\mathrm{HH}$ signaling activation. Thus, pAKT was not altered at all by oncRAS in none of the cell lines, whereas oncRAS strongly induced phosphorylation of ERK in RMS-13, but not in RUCH-2 cells. 


\subsubsection{MEK activity inhibits proliferation of oncRAS-transduced RUCH-2 cells}

RAS signaling is known to be involved in various cellular processes including proliferation and metabolic activity. In order to investigate the effects of oncRAS on the proliferative capacity and metabolic activity in human RMS cell lines BrdU and WST-1 assays were performed, respectively. As shown in Figure 16A and B oncRAS decreased the proliferative capacity and metabolic activity in RUCH-2 cells, whereas it increased it in RMS-13 (Figure $16 \mathrm{~B}$ und $\mathrm{D})$.

A

RUCH-2

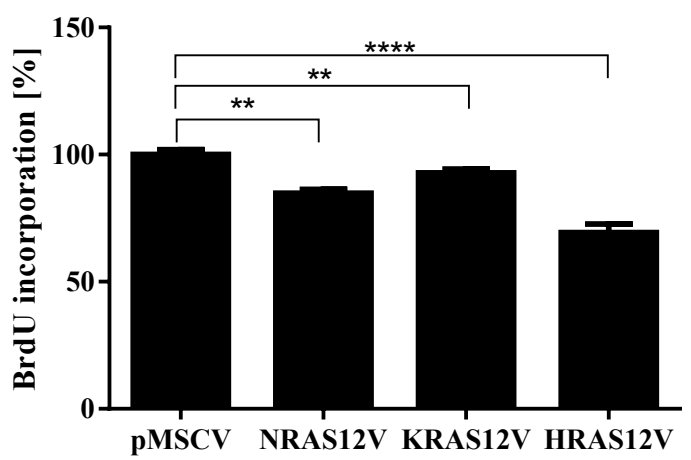

C

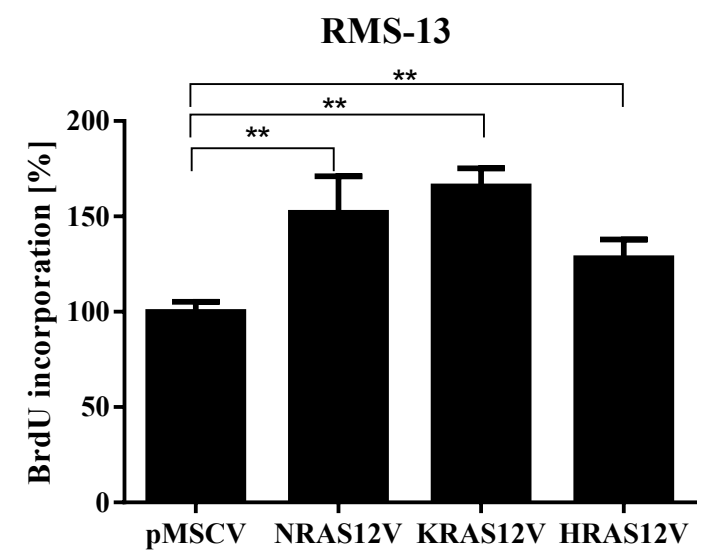

B

\section{RUCH-2}

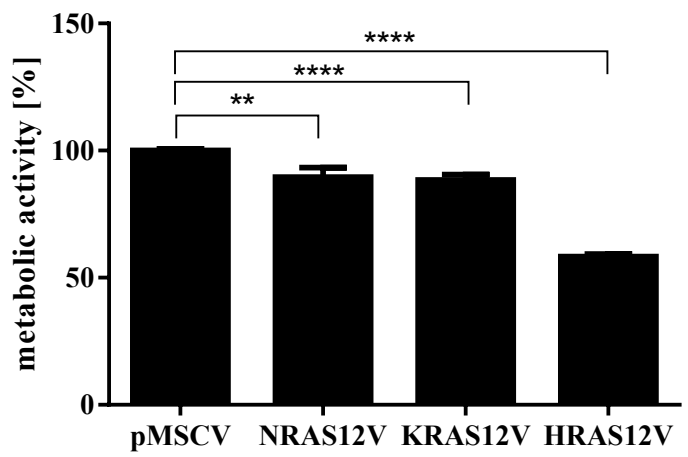

D

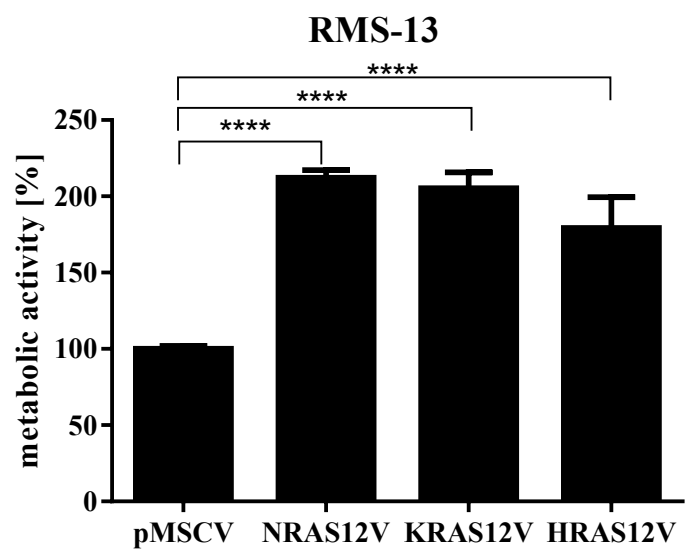

Figure 16: Modulation of the proliferative capacity and metabolic activity in oncRAS transduced RMS cell lines. Quantification of the proliferative capacity measured by BrdU assay and metabolic activity by WST-1 of (A and B) RUCH-2 and (C and D) RMS-13 cells stably transduced with either pMSCVpuro (pMSCV), pMSCVpuro-NRAS12V (NRAS12V), pMSCVpuro-KRAS12V (KRAS12V), or pMSCVpuroHRAS12V (HRAS12V), as indicated. BrdU incorporation and metabolic activity of the pMSCVpurotransduced cells was set to $100 \%$. All data represent at least 3 independent experiments measured in 
triplicates displayed as mean \pm SEM. Statistical significance was tested by using Mann-Whitney test. * $P<0.05$, ** $P<0.005, * * * P<0.0005$ and $* * * * P<0.0001$

In order to investigate if canonical $\mathrm{HH}$ signaling, RAF/MEK/ERK signaling or PI3K signaling are involved in the changes of the proliferation and metabolic activity behavior in RMS cells, the cells were incubated for $24 \mathrm{~h}$ with HhA, UO126 and PI103. When RUCH-2 cells were incubated with HhA and PI103, proliferation and metabolic activity were decreased in all settings independently of the RAS activity (Figure 17). However, UO126 significantly increased metabolic activity in RUCH-2 transduced with oncogenic NRAS and KRAS and the proliferative capacity in NRAS-transduced cells to the basal levels detected in control RUCH2 cells. This indicates that in RUCH-2 transduced with oncogenic NRAS or KRAS it is MEK activity that decreases the metabolic capacity. In addition, MEK activity also inhibits the proliferative capacity of NRAS- transduced cells. The data also suggest that MEK inhibitors are probably not a good treatment option in ERMS harboring oncogenic RAS mutations. 
A

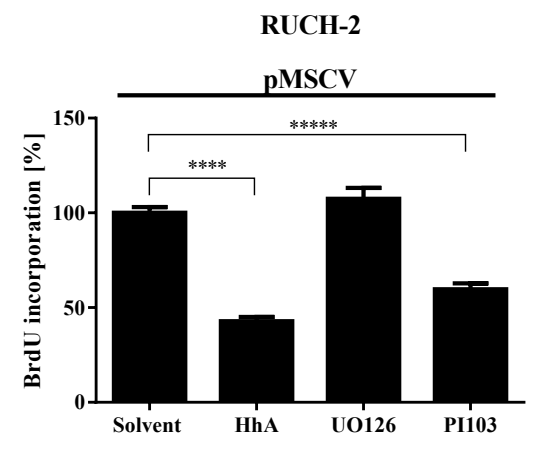

D

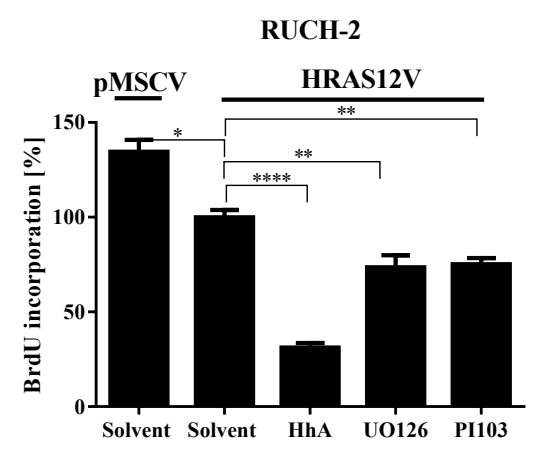

G

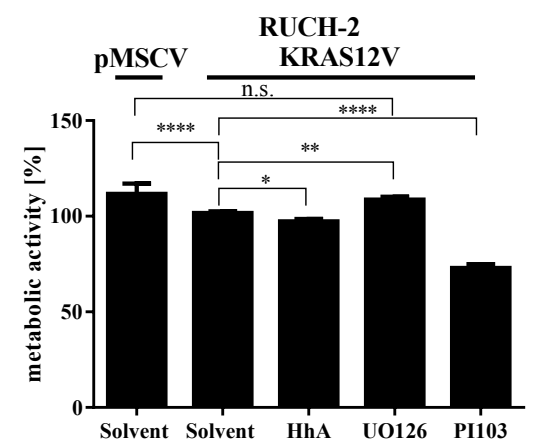

B

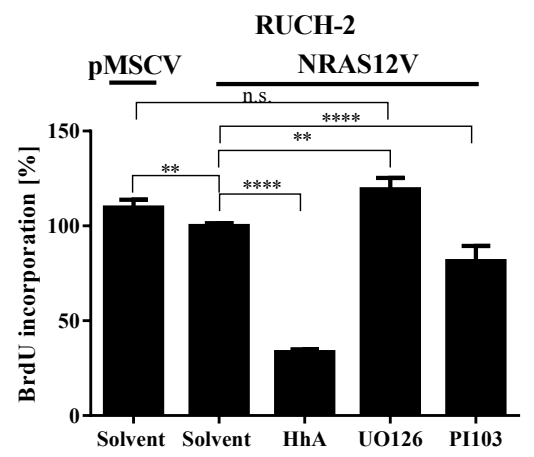

$\mathbf{E}$

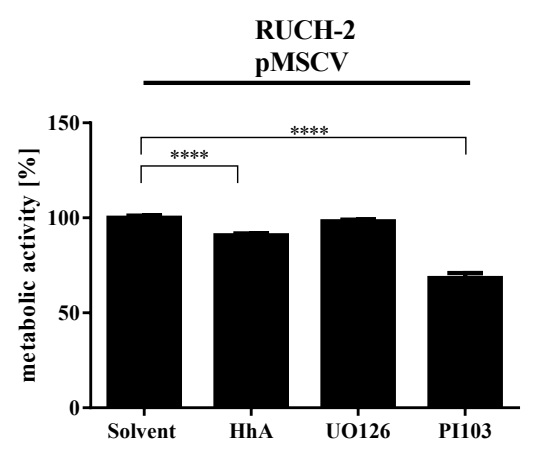

$\mathbf{H}$

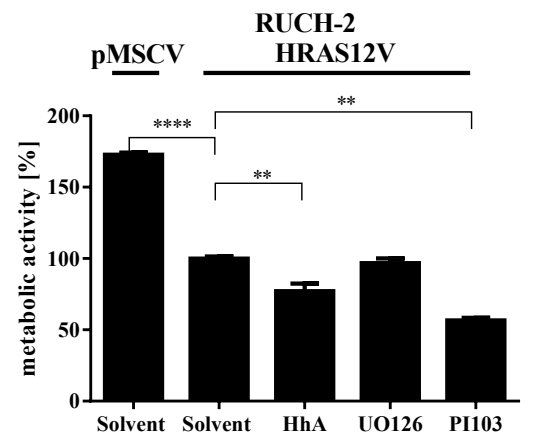

C

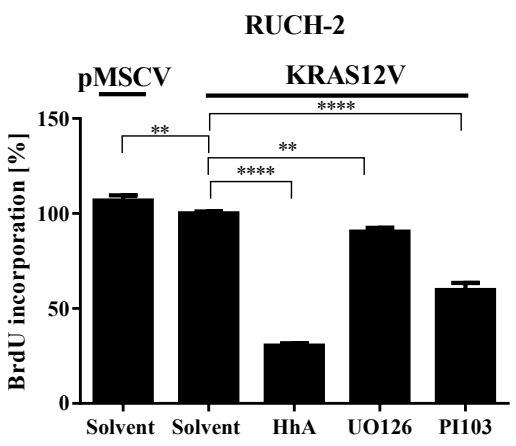

$\mathbf{F}$

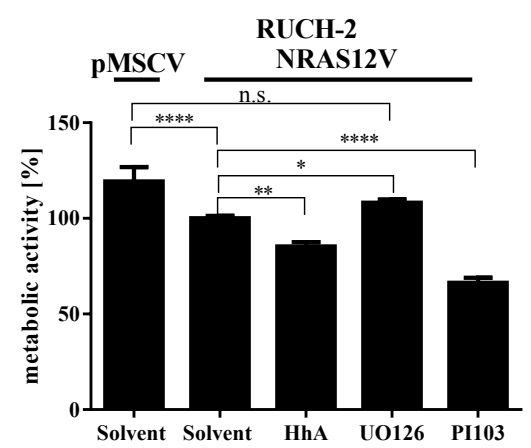

Figure 17: oncRAS modulates the proliferative capacity and metabolic activity ERMS. Quantification of the proliferative capacity measured by BrdU assay (A-D) and metabolic activity by WST-1 (F-H) of RUCH-2 cells stably transduced with either pMSCVpuro (pMSCV), pMSCVpuro-NRAS12V (NRAS12V), pMSCVpuro-KRAS12V (KRAS12V), or pMSCVpuro-HRAS12V (HRAS12V), as indicated. Cells were treated for $24 \mathrm{~h}$ with solvent, HhA, UO126 or PI103 for 24h. The proliferation rates and metabolic activity of the solvent controls were set to $100 \%$ (please note that in the figures showing data of solvent treated control and oncRAS cell lines, the data of solvent treated oncRAS cell lines were set to $100 \%$ ). All data represent at least 3 independent experiments measured in triplicates displayed as mean \pm SEM. Statistical significance was tested by using Mann-Whitney test. ${ }^{*} \mathrm{P}<0.05$, ** $\mathrm{P}<0.005$, *** $\mathrm{P}<0.0005$ and **** $\mathrm{P}<0.0001$. Please note that the depiction of significance is equal as in Figure 12. 
A

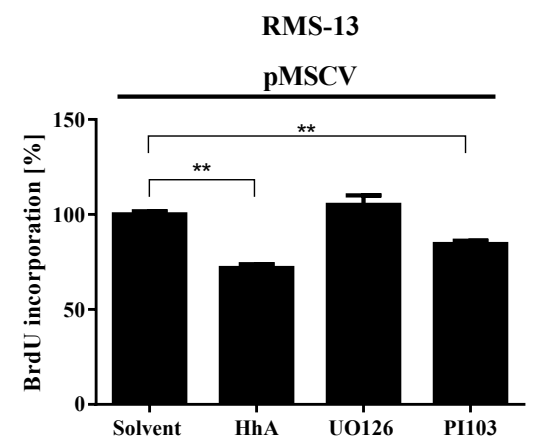

D

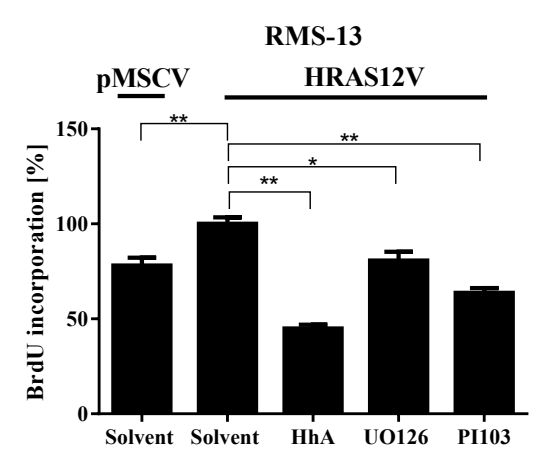

G

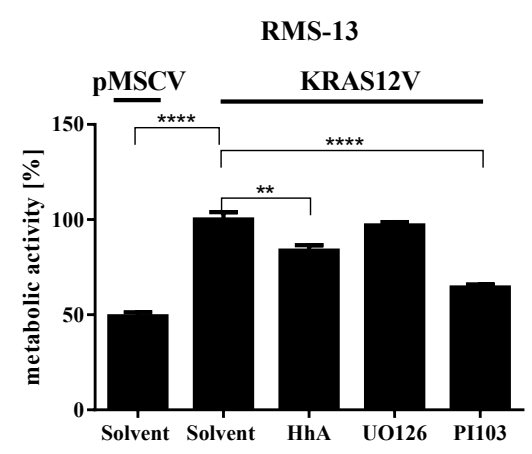

B

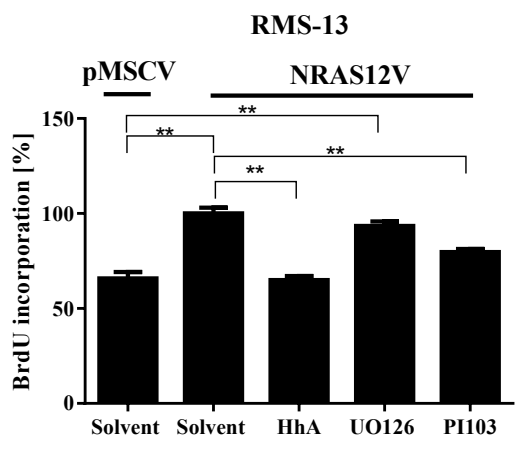

$\mathbf{E}$

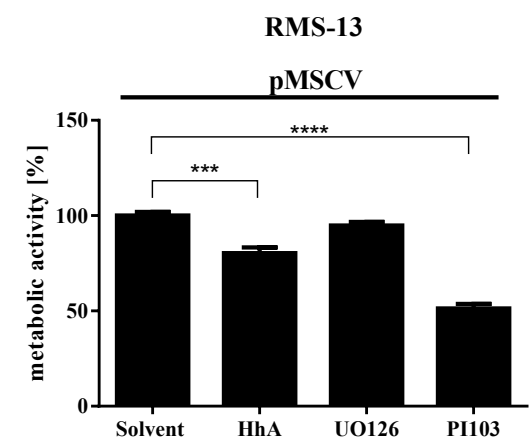

$\mathbf{H}$

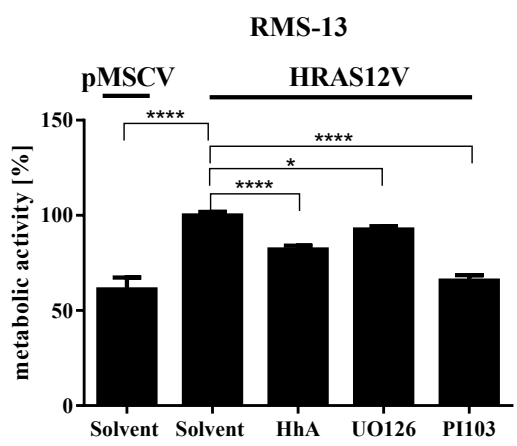

C

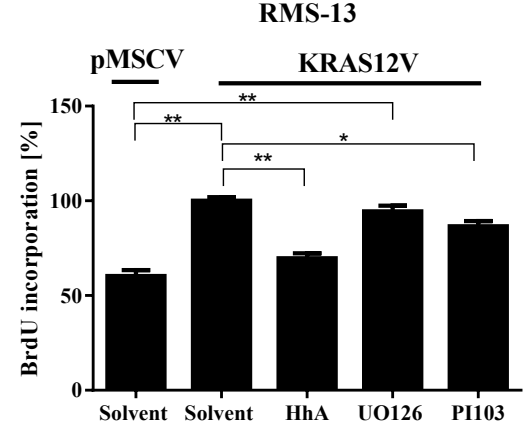

$\mathbf{F}$

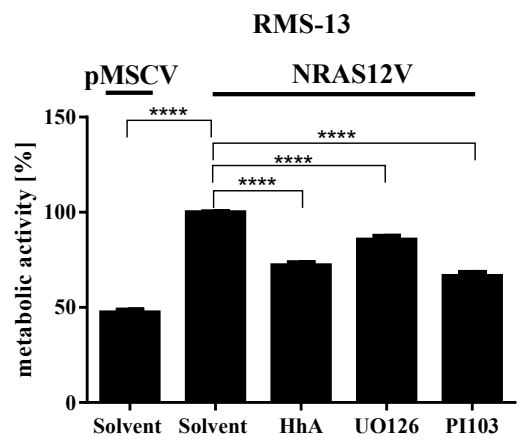

Figure 18: oncRAS modulates the proliferative capacity and metabolic activity in ARMS. Quantification of the proliferative capacity measured by BrdU assay (A-D) and metabolic activity by WST1 (F-H) of RMS-13 cells stably transduced with either pMSCVpuro (pMSCV), pMSCVpuro-NRAS12V (NRAS12V), pMSCVpuro-KRAS12V (KRAS12V), or pMSCVpuro-HRAS12V (HRAS12V), as indicated. Cells were treated for $24 \mathrm{~h}$ with solvent, HhA, UO126 or PI103 for $24 \mathrm{~h}$. The proliferation rates and metabolic activity of the solvent controls were set to $100 \%$ (please note that in the figures showing data of solvent treated control and oncRAS cell lines, the data of solvent treated oncRAS cell lines were set to $100 \%)$. All data represent at least 3 independent experiments measured in triplicates displayed as mean \pm SEM. Statistical significance was tested by using Mann-Whitney test. ${ }^{*} \mathrm{P}<0.05,{ }^{* *} \mathrm{P}<0.005,{ }^{* * *} \mathrm{P}<0.0005$ and ${ }^{* * * *} \mathrm{P}<0.0001$ (depiction of significant values as in Figure 17). 
In RMS-13 cells the incubation with the above mentioned inhibitors revealed that HhA and PI103, but not UO126, can inhibit proliferation and metabolic activity of control cells (Figure 18). This indicates that proliferation of RMS-13 control cells may be regulated by $\mathrm{HH}$ and $\mathrm{PI} 3 \mathrm{~K} / \mathrm{mTOR}$ signaling. HhA and PI103 also repressed proliferation and metabolic activity in RMS-13 cells that express oncogenic NRAS, KRAS and HRAS. This was different for UO126 that not consistently repressed the proliferative capacity and metabolic activity of oncRAS-transduced RMS-13 cells.

\subsubsection{0. oncRAS slightly induces muscle differentiation in RMS-13 cells}

Since it is known that oncogenic RAS signaling is involved in processes of differentiation (Haigis et al. 2008), the changes in the expression of muscle differentiation markers were measured by qRT PCR reaction. As shown in Figure 19 all 3 oncRAS slightly increased the expression levels of the muscle differentiation markers MYOD and DESMIN in RMS-13 cells. However the increase was not significant. This indicates that oncRAS may induce a slightly more differentiated phenotype in RMS-13 cells.

Unfortunately the ERMS cell line RUCH-2, does not or merely expresses muscle differentiation markers, which were also not upregulated by oncRAS.

A

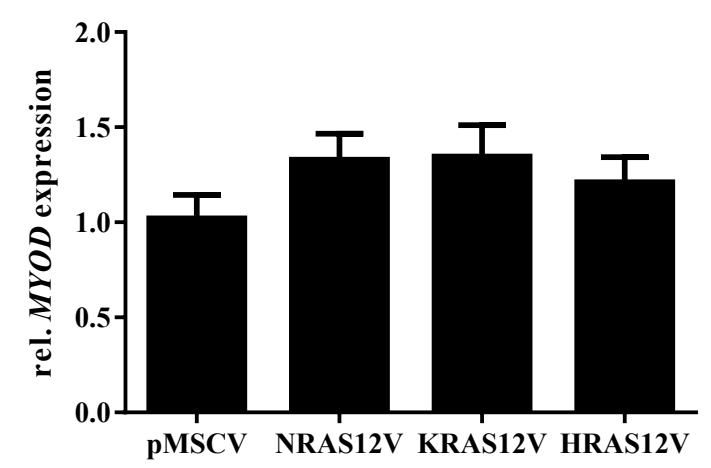

B

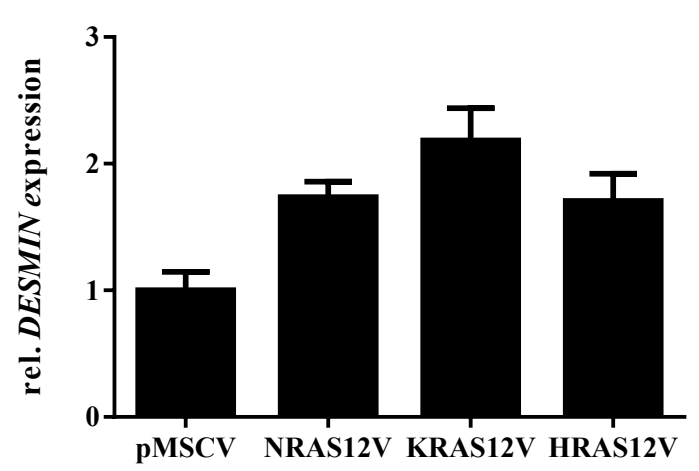

Figure 19: Slightly increased muscle differentiation in oncRAS-transduced RMS-13 cells. Quantification of (A) MYOD and (B) DESMIN expression levels measured by qRT-PCR of RMS-13 stably transduced with either pMSCVpuro (pMSCV), pMSCVpuro-NRAS12V (NRAS12V), pMSCVpuroKRAS12V (KRAS12V), or pMSCVpuro-HRAS12V (HRAS12V), as indicated. The expression levels of the oncRas transduced cells were normalized to expression in RMS-13 pMSCV which was set to 1 . All data represent at least 2 independent experiments measured in triplicates displayed as mean \pm SEM. Statistical significance was tested by using Mann-Whitney test. 


\subsubsection{1. oncRAS-induced HH signaling inhibition in RMS-13 cells is not mediated by DYRK1B}

In the ARMS cell line RMS-13 oncRAS probably inhibited GLI1 expression manner independently of the MEK or the PI3K/mTOR axis. Thus, the inhibition may be mediated by a yet unknown factor or signaling cascade. A good candidate is DYRK1B that can be activated by RAS and that has been reported to block the function of GLI2 and to promote GLI3 repressor formation (Lauth et al. 2010). Surprisingly, and as shown in Figure 20 oncRAS expression decreases DYRK1B levels in RMS-13.

Next, DYRK1B was targeted with 2 specific siRNAs i.e. DYRK1B_01 and DYRK1B_02 siRNA from Dharmacon (D-004806-01 and D-004806-02).

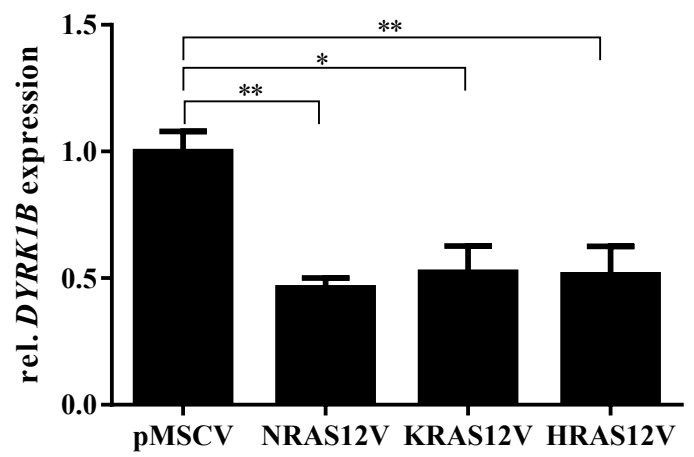

Figure 20: Downregulation of DYRK1B expression in oncRAS-transduced RMS-13 cells. Quantification of DYRK1B expression levels measured by qRT-PCR in RMS-13 cells stably transduced with either pMSCVpuro (pMSCV), pMSCVpuro-NRAS12V (NRAS12V), pMSCVpuro-KRAS12V (KRAS12V), or pMSCVpuro-HRAS12V (HRAS12V). Expression levels were normalized to $18 S$ rRNA levels. The expression levels of the oncRas transduced cells were normalized to expression in RMS-13 pMSCV which was set to 1 . All data represent at least 2 independent experiments measured in triplicates displayed as mean \pm SEM. Statistical significance was tested by Mann-Whitney test. $* \mathrm{P}<0.05$ and $* *$ $\mathrm{P}<0.005$

The DYRK1B knockdown significantly decreased DYRK1B expression in RMS-13 cells transduced with the control vector (pMSCV in Figure 21A). This decrease went along with an inhibition of the GLI1 expression (Figure 21B) and not - as expected - with an induction of GLI1. A similar result was revealed in oncRAS-transduced RMS-13 upon siRNA treatment. Although the decrease of DYRK1B expression in oncRAS-transduced RMS-13 was not significant it was accompanied by a decrease in GLII expression level. 
Unfortunately, due to shortage of time I was not able to repeat the experiment using different siRNAs that would have allowed for a more efficient knockdown of DYRK1B. Nevertheless, the coincidence of significant downregulation of both DYRKIB and GLII expression in oncRAS RMS-13 cells indicates that DYRK1B in RMS-13 activates GLII expression rather than inhibiting it. This would be different from human pancreatic cancer in which DYRK1B inhibits HH signaling activity (Lauth et al. 2010).

$\mathbf{A}$

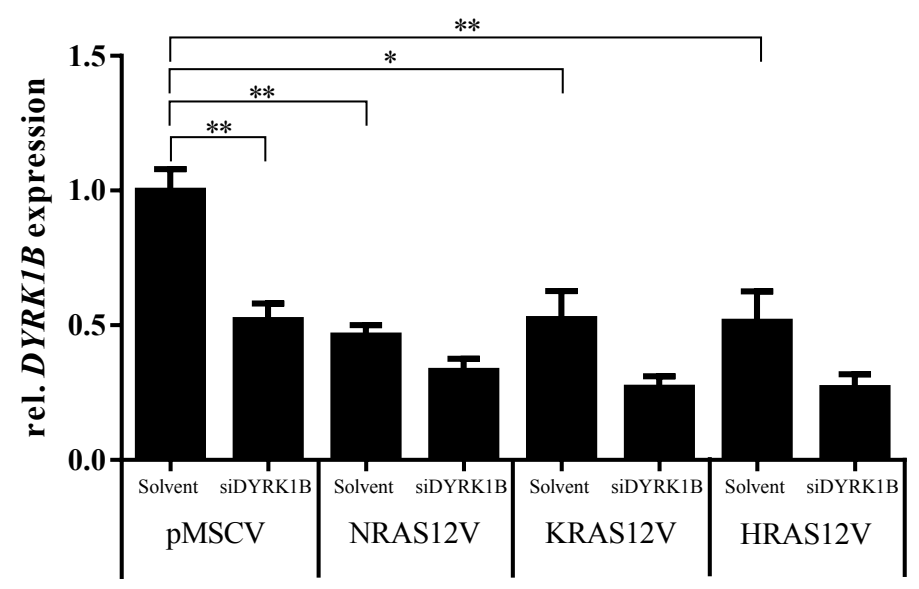

B

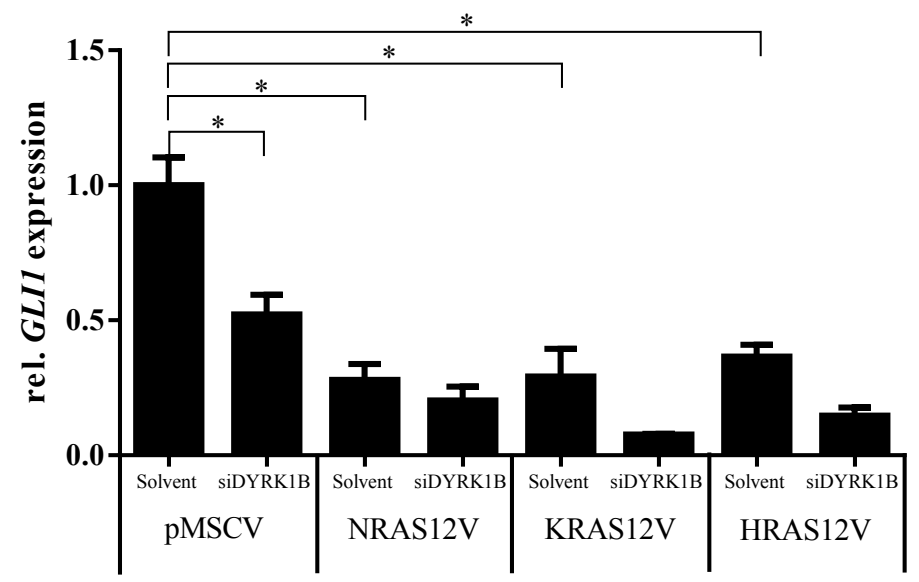

Figure 21: DYRK1B knockdown decreases the expression levels of GLI1 and DYRK1B in oncRAStransduced RMS-13 cells. Quantification of (A) DYRKIB and (B) GLII expression levels measured by qRT-PCR in RMS-13 cells stably transduced with either pMSCVpuro (pMSCV), pMSCVpuro-NRAS12V (NRAS12V), pMSCVpuro-KRAS12V (KRAS12V), or pMSCVpuro-HRAS12V (HRAS12V) without and with DYRK1B knockdown. Expression levels were normalized to $18 S$ rRNA levels. All expression levels were normalized to expression of solvent treated RMS-13 pMSCV cells which was set to 1. All data measured in triplicates displayed as mean \pm SEM. Statistical significance was tested by using MannWhitney test. Significances are discussed in the text. 


\subsection{Growth of oncRas-transduced RMS cell lines in nude mice}

Additionally to the above mentioned in vitro analysis of human RMS cell lines, the cells were transplanted in nude mice. This was done to investigate if oncRAS influence the growth behavior of human RMS cell lines in vivo.

\subsection{1. oncRAS change the tumor growth behavior of RMS tumor xenografts}

RMS cells were injected into the flanks of 8 weeks old nude mice (see chapter 4.17.6). To circumvent individual mouse effects that might have influenced the growth of control- and oncRAS-transduced cell lines, each mouse was injected with pMSCVpuro transduced RMS cells on one side and with either pMSCVpuro-NRAS12V or pMSCVpuro-KRAS12V transduced RMS cells into the other side. When the tumors were visible, the size was measured 5 to 7 times a week.

Unfortunately and unexpectedly, the transplantation of RUCH-2 (ERMS) cells was unsuccessful, because the injected tumor cells didn't form tumors at all, even when a higher number of cells were injected.

When oncRAS-transduced RMS-13 cell lines were transplanted both oncogenic KRAS and NRAS induced a more aggressive tumor growth. This was demonstrated by increased tumor size and weight (Figure 22). Indeed, the monitoring studies had to be stopped at day 18 $(N R A S 12 V)$ or at day $20(\mathrm{KRAS12V})$ after transplantation. Thus, the tumor size of the oncRAS RMS-13 xenografts reached a diameter of $1.5 \mathrm{~cm}$, which is an abort criteria in transplantation studies of tumor cells. Within this time frame most of the control xenografts derived from pMSCVpuro-transduced RMS-13 cells did not grow. Therefore, only very small tumors could be isolated, which hampered further molecular analyses of RNA or protein lysates. Taken together, these results indicate that oncogenic NRAS and KRAS increase the tumor growth rate of human ARMS cells. This data fits to the in vitro data showing that both NRAS and KRAS induce a more aggressive growth behavior of RMS-13 cells (see Figure 22). 
A

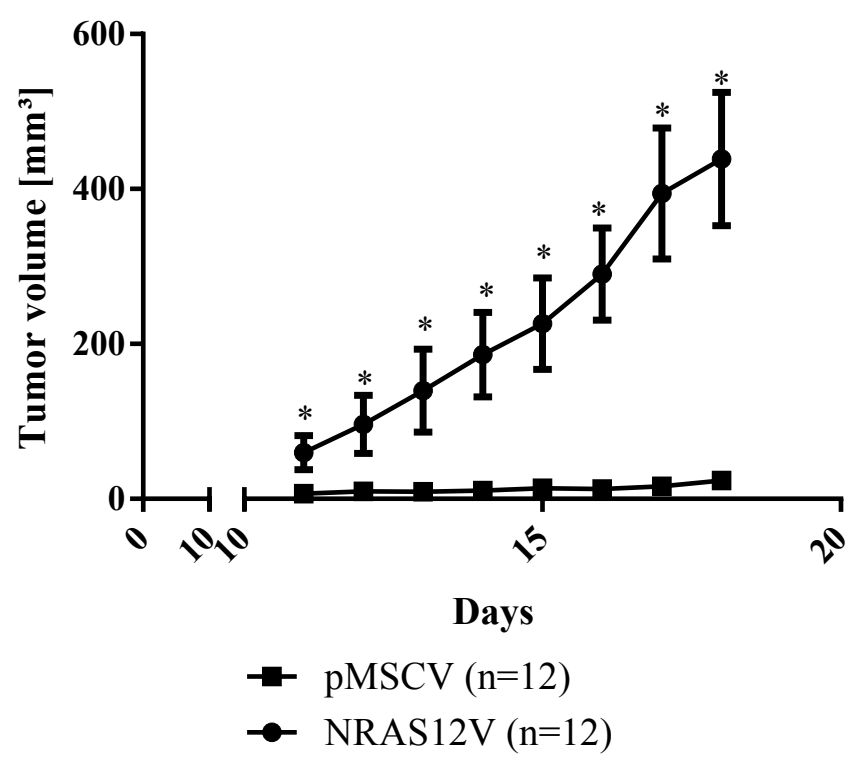

C

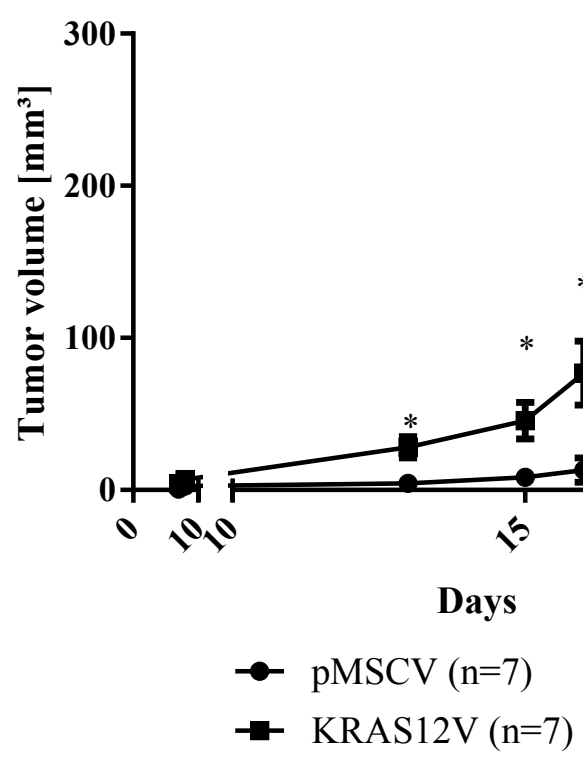

B

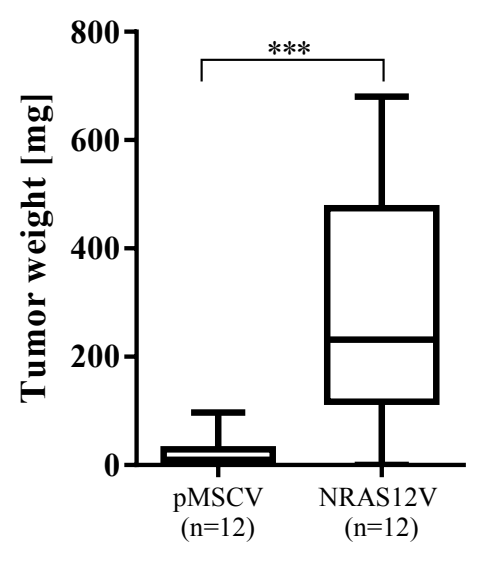

D

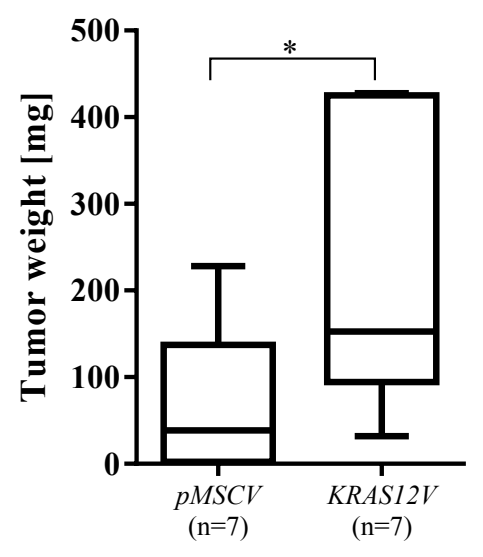

Figure 22: oncRAS enhances tumor growth of RMS-13 cells. $5 \times 10^{6}$ cells in a final volume of $100 \mu 1$ were injected into the flanks of 8 weeks old nude mice. When the tumors were visible the size was measured with a caliper. At the end tumors were isolated and weighted. (A) Growth curve and (B) tumor weight of the tumors derived from RMS-13 xenografts that have been transduced with pMSCVpuro (pMSCV) or pMSCVpuro-NRAS12V (NRAS12V). (C) Monitoring curve and (D) tumor weight of the tumors derived from RMS-13 xenografts that have been transduced with pMSCVpuro (pMSCV) or pMSCV puro-KRAS12V (KRAS12V). All data are displayed as mean \pm SEM. Statistical significance of the tumor volume was tested by Wilcoxon test and of the tumor weight by using unpaired $t$ test with Welch's correction. ${ }^{*} P<0.05$, ${ }^{* *} P<0.005$ and $* * * P<0.0005$. 


\subsection{2. oncRAS does not obviously influence the morphology of RMS xenografts}

To investigate if oncRAS influenced the morphology of tumor xenografts from RMS-13 cells, the tumors were embedded in paraffin, sliced into sections, mounted on a slide and stained by H\&E staining (see chapter 4.16.5). As illustrated in Figure 23, oncRas in RMS-13 does not obviously change the tissue structure.

A

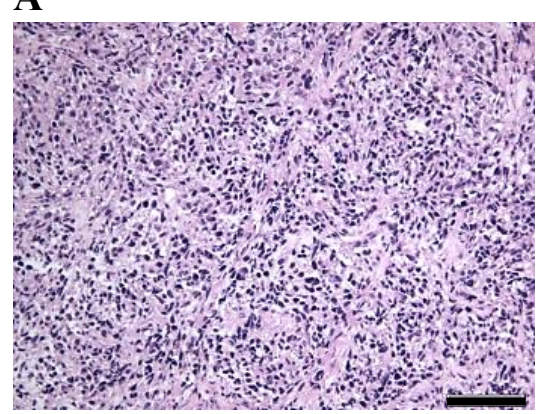

B

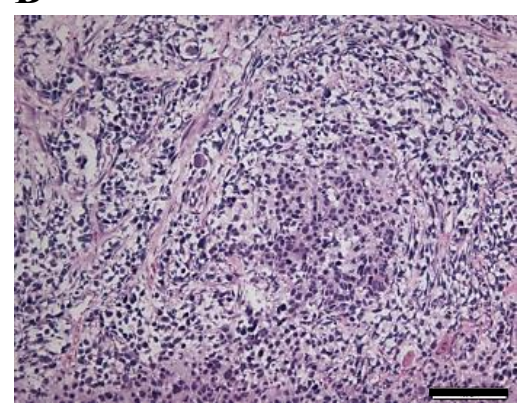

C

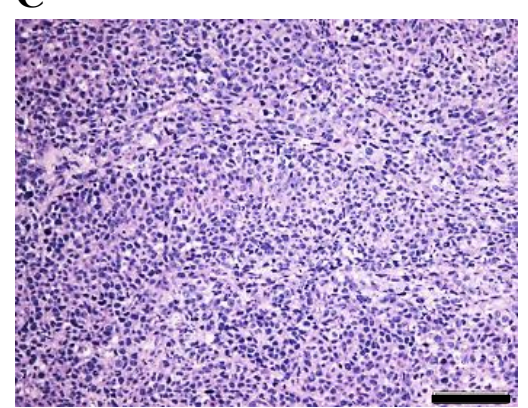

Figure 23: Histology of oncRAS-transduced RMS-13 xenografts. Representative pictures from H\&E stained tumors derived from RMS-13 cells that have been stably transduced with (A) pMSCVpuro (B) pMSCVpuro-NRAS12V or (C) pMSCVpuro-KRAS12V, Scale bar $=100 \mu \mathrm{m}$. 


\subsection{Growth of RMS after conditional expression of oncRas in Ptch mutant mice}

\subsubsection{Generation and identification of $\operatorname{Ptch}^{\mathrm{del/+}}$ oncRas $^{\mathrm{fl} /-} \mathrm{Myf5creERT^{tg/- }}$ mice}

As already explained in the introduction, $\mathrm{Ptch}^{\mathrm{del} /+}$ mice were used as an in vivo mouse model for RMS. Figure 24 shows the wt and mutant Ptch locus and the location of the primers used for genotyping tail biopsies of $\mathrm{Ptch}^{\mathrm{del} /+}$ mice.

A

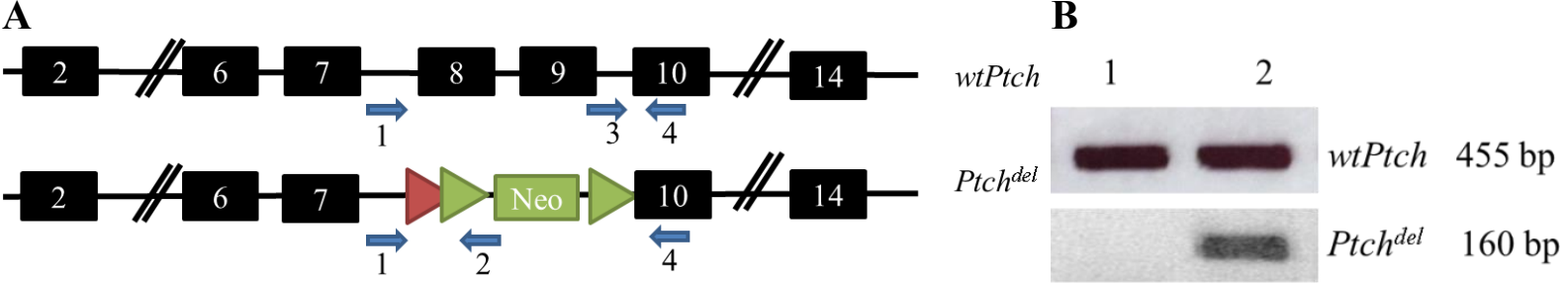

Figure 24: Scheme of the Ptch locus and genotyping of heterozygous $P t c h{ }^{d e l}$ mice. (A) The figure shows the wtPtch allele and the mutant $P t c h{ }^{d e l}$ allele in which the floxed Exon 8 and 9 were deleted. The primer locations are indicated as blue arrows $(1=\mathrm{mPtcdelNx}-\mathrm{F}, 2=\mathrm{mPtcNxR}, 3=\mathrm{mPTCNx} F \mathrm{~F}$ and $4=$ mPTCwt_R). The figure was adapted from (Uhmann et al. 2007). (B) The primer pairs mPtcdelNx-F (1) and mPtcNxR (2) and mPTCNx_F (3) and mPTCwt_R (4) indicated in panel A were used to identify the Ptch $^{\text {del }}$ allele (160 bp) and wtPtch allele (455 bp), respectively, by PCR performed on mouse tail DNA. Line 1 shows genotyping of a homozygous wtPtch mouse and line 2 that of a heterozygous Ptch $^{\text {del/+ }}$ mouse.

As shown by our working group, $\operatorname{Ptch}^{\text {del/ }+}$ mice develop RMS depending on the genetic background. On a mixed C57BL/6 x BALB/c (B6xBALB $50 \%: 50 \%$ ) background approximately $60 \%$ of $\mathrm{Ptch}^{\mathrm{del} / \mathrm{+}}$ mice develop this tumor, whereas inactivation of one Ptch allele on a pure B6 background does not result in RMS formation. On a pure BALB background approximately $90 \%$ of Ptch $^{\text {del/+ }}$ develop RMS. In order to see whether the expression of oncRas genes has an impact on RMS incidence, latency time or multiplicity a mixed B6xBALB background was anticipated. This was due to the fact that a RMS incidence of $60 \%$ allows for monitoring of an increase as well as a decrease in Ras-induced changes of the tumor incidence. For this purpose heterozygous $\mathrm{Ptch}^{\mathrm{del} /+}(100 \%$ BALB) mice were bred to mice that conditionally express oncogenic KRas (LSL-K-RASG12D(Tuveson et al. 2004)), HRas (FR-HRASG12V (Chen et al. 2009)) or NRas (NRAS LSL-G12D (Haigis et al. 2008)). All conditional Ras mutants were on a pure B6 background. Whereas LSL-K-RASG12D and $N R A S L S L-G 12 D$ mice conditionally express the murine RasG12D variant, FR-HRASG12V mice conditionally express the murine RasG12V variant (see Figure 25). The conditional 
expression of the respective Ras variants is accomplished in the presence of a cre recombinase, that removes a floxed stop cassette in $N R a s G 12 D^{\mathrm{f} / /}$ and $K R a s G 12 D^{\mathrm{f} / /-}$ mice or the floxed wtRas locus in HRasG12 $\mathrm{V}^{\mathrm{fl} /-}$ mice (see Figure 25). This results in the expression of the oncogenic Ras variants.

A

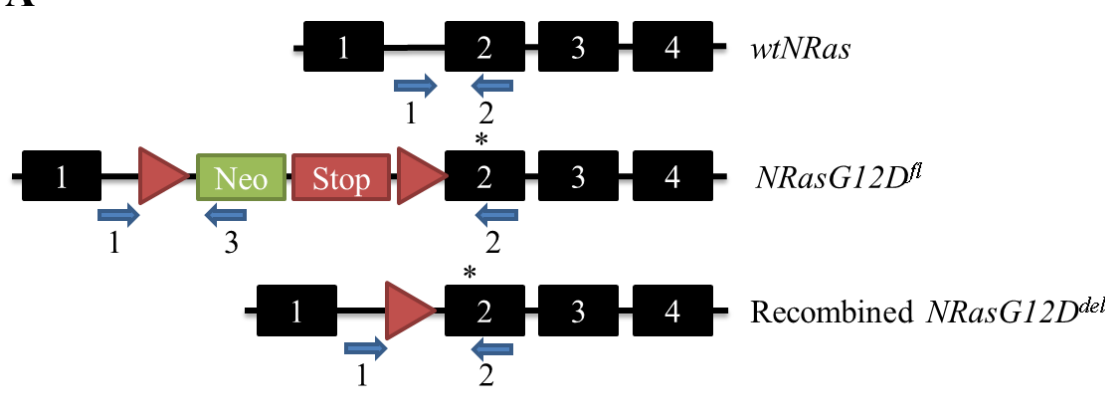

C

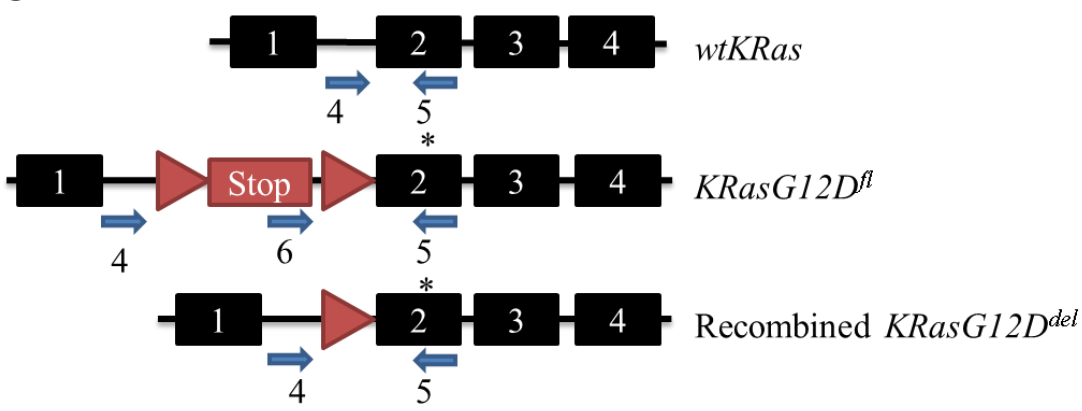

B

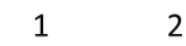

we we $487 \mathrm{bp}$ NRasG12D fl $345 \mathrm{bp}$

E
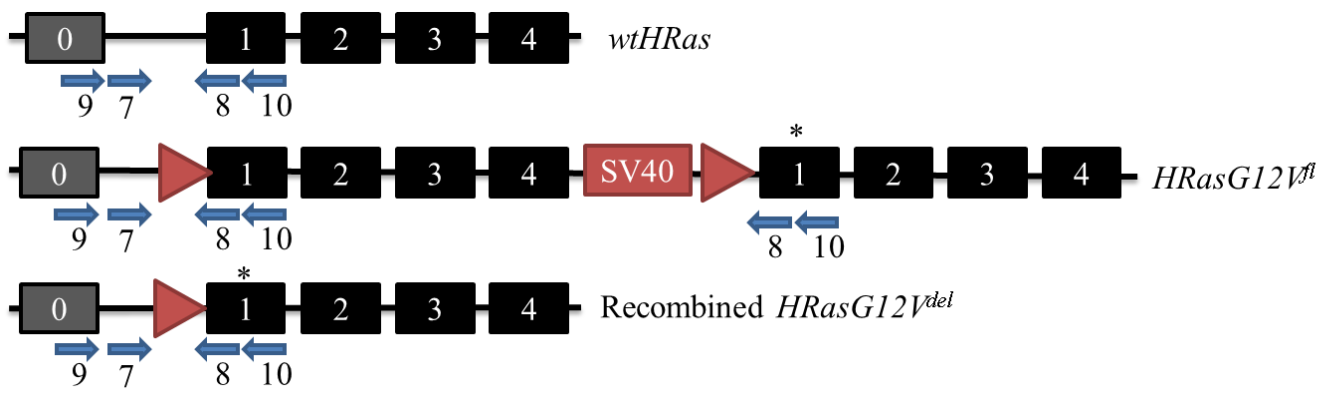

$\mathbf{F}$

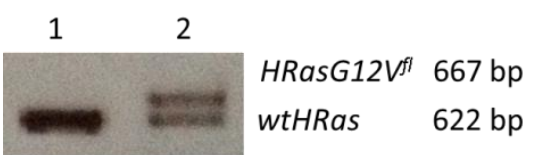

Figure 25: Scheme of the Ras loci and genotyping of conditional oncogenic Ras mice. (A) The figure shows the wtNRas allele, the floxed NRasG12D fl allele and the recombined $N R a s G 12 D^{\text {del }}$ allele. The floxed NRasG12D fl allele consists of a lox-stop-lox element (LSL) (red triangles and squared) which was inserted into the first intron, upstream of the G12D activating mutation in the second exon (Haigis et al. 2008). In the presence of a cre recombinase the stop element is excised and NRasG12D is expressed. The primer 
locations are indicated as blue arrows $(1=$ mNRas-WT-For, $2=$ mNRas-WT-Rev and $3=$ NRas-Mut-Rev $)$. The figure was adapted from (Haigis et al. 2008) (B) The primers mNRas-WT-For (1), mNRas-WT-Rev (2) and NRas-Mut-Rev (3) indicated in panel A identify the genomic wtNRas allele (487 bp) and the $N R a s G 12 D^{f l}$ allele (345 bp) by PCR performed on mouse tail DNA. Line 1 shows genotyping of a homozygous wtNRas mouse and line 2 that of a heterozygous NRasG12D $D^{\mathrm{f} /}$ mouse. (C) The figure shows the $w t K R a s$ allele, the floxed $K R a s G 12 D^{f l}$ allele and the recombined $K R a s G 12 D^{\text {del }}$ allele. The floxed $K R a s G 12 D^{f l}$ allele consists of a lox-stop-lox element (LSL) (red triangles and squared) which was inserted into the first intron, upstream of the G12D activating mutation in the second exon. In the presence of a cre recombinase the stop element is excised and KRasG12D is expressed. The primer locations are indicated as blue arrows $(4=$ Kras-WT_UP1, $5=$ Kras-URP_Lp1 and $6=$ KrasG12Dmut_UP). The figure was adapted from (Tuveson et al. 2004) (D) The primers Kras-WT_UP1 (4), Kras-URP_Lp1 (5) and KrasG12Dmut_UP (6) indicated in panel $\mathrm{C}$ were used to identify the wtKRas allele (270 bp) and the KRasG12D ${ }^{f l}$ allele $(170 \mathrm{bp}$ ). Line 1 shows genotyping of a homozygous wtKRas mouse and line 2 that of a heterozygous KRasG12 $D^{\mathrm{f} /} /$ mouse. (E) The figure shows the wtHRas allele, the floxed HRasG12 $V^{f l}$ allele and the recombined HRas G12 $V^{d e l}$ allele. The floxed HRasG12 $V^{f l}$ allele consists of a floxed (red triangles) wtHRas followed by a complete mutant $H R a s G 12 \mathrm{~V}$ gene. The respective vector was inserted into the first intron of the wt Hras locus. In the presence of a cre recombinase the floxed wtHRas sequence is excised and HRasG12V is expressed. The primer locations are indicated as blue arrows $(7=\mathrm{eCreRASF}, 8=\mathrm{eCreRasR}$, $9=\mathrm{mHRasG12Vrel} \mathrm{F1}$ and $10=\mathrm{mHRasG12Vrel} \mathrm{R1).} \mathrm{The} \mathrm{primers} 9$ and 10 are used for the quantification from cDNA for the recombination assay (see Figure 26C). The figure was adapted from (Chen et al. 2009) (F) The primers eCreRASF (7) and eCreRasR (8) indicated in panel E were used to identify the wtHRas allele $(622 \mathrm{bp})$ and the HRasG12 $\mathrm{V}^{f l}$ allele $(667 \mathrm{bp})$. Line 1 shows genotyping of a homozygous wtHRas mouse and line 2 that of a heterozygous HRas $G 12 \mathrm{~V}^{\mathrm{fl} / \mathrm{L}}$ mouse.

$M y f 5 c r e E R T^{t g /-}$ mice express a tamoxifen-inducible Cre recombinase under the control of Myf5 promoter (see Figure 26) which is expressed in RMS (Figure 29). For this purpose the Ptch $^{\text {del/+ }}$ oncRas $^{f l /-}$ mice were crossed to Myf5creERT ${ }^{\text {tg/- }}$ mice that were on a mixed background (50 \% BALB, $25 \%$ B6, $12.5 \%$ FVB, $12.5 \%$ SV129) (see Figure 26). 
A

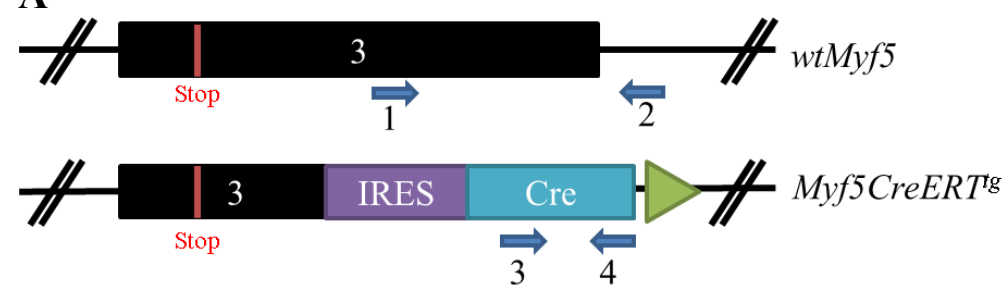

B

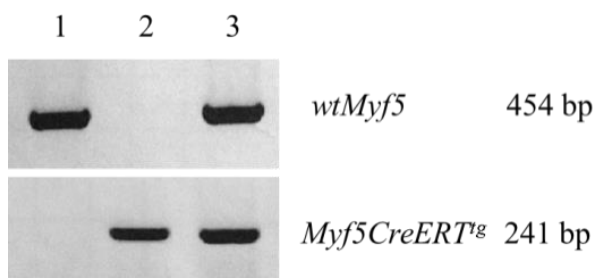

Figure 26: Scheme of the Myf5 locus and genotyping of the inducible Myf5CreERT mice. (A) The figure shows the $w t M y f 5$ allele and the Myf5creERT $T^{t g}$ allele. For the Myf5CreER mouse line, a targeting vector was constructed that placed an ires-CreER ${ }^{\text {TM}}$-FRT-Neo-FRT cassette in the $3^{\prime}$ untranslated region of the Myf5 gene following the stop codon in exon3. The FRT (green triangle) flanked Neo-cassette was flipped out resulting in the left FRT-site behind the CreERT insert. The primer locations are indicated as blue arrows $(1=\mathrm{cK} 382,2=\mathrm{ck} 383,3=\mathrm{c}-\mathrm{F}$ and $4=\mathrm{c}-\mathrm{R})$. The figure was adapted from (Biressi et al. 2013). (B) The primer pairs cK382 (1) and cK383 (2) and c-F (3) and c-R (4) indicated in panel A identify the genomic wtMyf5 allele ( $454 \mathrm{bp}$ ) and the Myf5creERT ${ }^{g}$ allele ( $241 \mathrm{bp}$ ) by PCR performed on mouse tail DNA. Line 1 genotyping of a $w t M y f 5$ mouse, line 2 genotyping of a homozygous $M y f 5 \mathrm{creER} T^{\mathrm{tg} / \mathrm{tg}}$ mouse and line 3 that of a heterozygous $M y f 5 c r e E R T^{\text {tg/ }}$ mouse.

In the resulting Ptch $^{\text {del/ }}$ oncRas $^{f l /-}$ Myf5CreERT ${ }^{\text {tg/- }}$ offspring (50\% BALB, $37.7 \%$ B6, $6.25 \%$ FVB, $6.25 \%$ SV129) the expression of oncogenic NRas, KRas or HRas was induced at an age of 4 weeks by tamoxifen. The mice were monitored weekly for up to 200 days for RMS formation. A schematic representation of the monitoring study is shown in Figure 27.

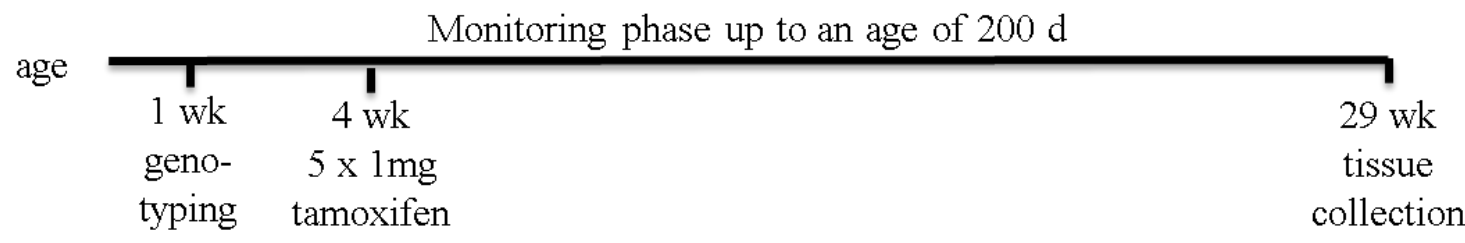

Figure 27: Schematic representation of the schedule for mouse monitoring. The Ptch $^{\mathrm{del/}+}$ oncRas $^{\mathrm{fl} /-}$ Myf5CreERT $T^{\mathrm{g} / \mathrm{-}}$ mice were biopsied at the age of 1-7 days, genotyped and half of them were treated at an age of 4 weeks with $1 \mathrm{mg}$ tamoxifen on 5 consecutive days. Untreated Ptch $^{\text {del//+}}$ oncRas $^{\text {fl/ }}$ Myf 5 CreERT $T^{\text {tg/- }}$ served as controls. Both cohorts were monitored for up to an age 200 days and sacrificed. All tumors and the reference tissue (skeletal muscle) were isolated.

To test the extent of the Cre recombinase induction, Myf5creERT $T^{t /+}$ mice were crossed with the $R 26 R$ reporter mice. The $R 26 R$ reporter mice express a Cre-inducible $L a c Z$ gene under the control of the endogenous Rosa26 promoter. The expression of the $\beta$-galactosidase gene was 
investigated in double heterozygous $M y f 5 c r e E R T^{t g /+} R 26 R^{+/-}$mice after the injection (i.p.) of $5 \mathrm{mg}$ tamoxifen for five consecutive days. One week after the first injection the tissue was isolated kyro-embedded and the activity of the Myf5creERT was detected by X-Gal staining (Figure 28)

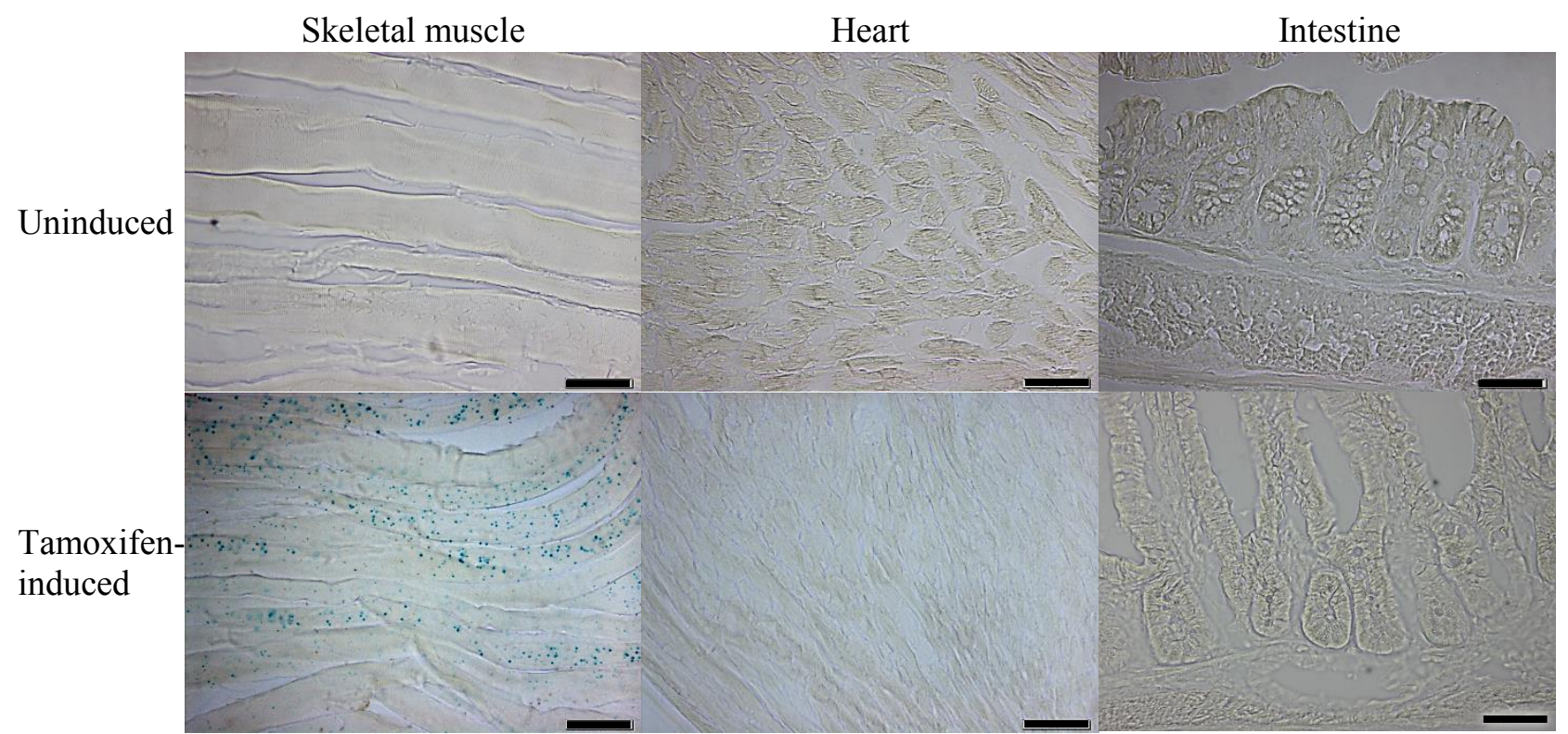

Figure 28: Tamoxifen-induced activity of the Myf5creERT in R26R-reporter mice. X-Gal-stainings of kryo-sections of tissues isolated from uninduced and tamoxifen-induced $M y f 5 c r e E R T^{g / t} R 26 R^{+/}$mice. Scale bar $=50 \mu \mathrm{m}$.

The skeletal muscle, heart and intestine were isolated from uninduced and tamoxifen-induced Myf5creERT $T^{t g /+} R 26 R^{+/-}$mice. Especially in the skeletal muscle of tamoxifen-induced mice a weak blue staining could be detected, whereas heart and intestine were negative. Furthermore I also planned to investigate the extent of the Cre induction in RMS. For this purpose heterozygous $\mathrm{Ptch}^{\mathrm{del} /+} \mathrm{Myf} 5 \mathrm{creER} \mathrm{Tg}^{\mathrm{tg} /+} \mathrm{R} 26 \mathrm{R}^{+/-}$mice were generated. Unfortunately, so far these mice have not developed any RMS.

However, to confirm recombination at the Ras loci after tamoxifen-mediated Cre induction, gDNA from skeletal muscle and RMS of uninduced and tamoxifen-induced

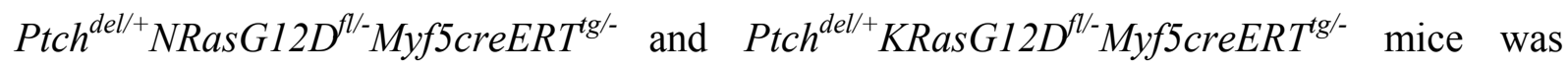
isolated (see chapter 4.15.1). The gDNA was analyzed by PCR (see chapter 4.15.5) with the primer pairs mNRas-WT-For and mNRas-WT-Rev for Ptch $^{\text {del/ }} N$ Ras $G 12 D^{f l /-}$ Myf5creERT $T^{t g /-}$ mice and Kras-WT_UP1 and Kras-URP_Lp1 for Ptch ${ }^{d e l /+}$ KRasG12 $D^{f l /-}$ Myf5creERT $T^{t g /-}$ mice. As seen in Figure 29A and B, in skeletal muscle only the wt band was detected from both 
tamoxifen-induced and none-induced mice. In addition, the wt band was also detected in RMS from none-induced mice. The wt band has a size of $487 \mathrm{bp}$ for $\operatorname{Ptch}^{\text {del/+ }} N$ RasG $12 D^{f l /-}$

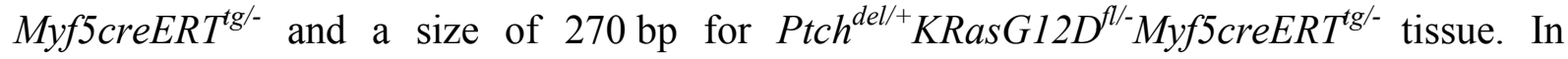
contrast the PCR (using the same primers) on the recombined gDNA results in a 30 bp larger band (see Figure 25 panel A and C recombined NRasG12D ${ }^{\text {del }}$ and KRasG12D ${ }^{\text {del }}$ allele), which is due to the remaining LoxP site after successful recombination. This band was only detected in RMS of mice that have been treated with tamoxifen. This shows that Myf5creERT is not leaky and that it is indeed strongly expressed in RMS.

Confirmation of the recombination at the floxed HRas locus was not possible by PCR on gDNA. For the testing cDNA from SM and RMS of tamoxifen-induced (oncHRas) and uninduced (wtHRas) Ptch ${ }^{\text {del/t+}} H R a s G 12 V^{f l /-}$ Myf5creERT ${ }^{t g /-}$ mice was amplified by PCR (see chapter 4.15.5) with the primer pair mHRasG12Vrel F1 and mHRasG12Vrel R1 (for primer location see Figure 25E primer 9 and 10). After amplification the PCR product was digested with BpmI. Since the G12V mutation destroys a BpmI site within the HRas sequence BpmI only digests wtHRas but not oncHRas cDNA. We would have expected to see just the digested fragments at $230 \mathrm{bp}$ and $72 \mathrm{bp}$ in uninduced mice and in tamoxifen-induced mice also the undigested one at $302 \mathrm{bp}$. However, as seen in Figure 29C the digested of the PCR product from SM and RMS cDNA of uninduced and tamoxifen-induced mice results in all three bands. Thus, suggesting that this system seems to be leaky. Nevertheless, the undigested band could also be detected in wt mice without a HRasG12 V allele (data not shown), indicating that there seems to be an error in the experimental setup.

Taken together these results indicate that the recombination at the NRas and KRas loci after tamoxifen-mediated Cre induction in induced Ptch $^{\text {del/ }}$ oncRas $^{\mathrm{fl} / /}$ Myf5 $\mathrm{creERT}^{\mathrm{tg} / \text { mice }}$ was successful in RMS, whereas the recombination at the HRas loci remained to be discovered. 
A

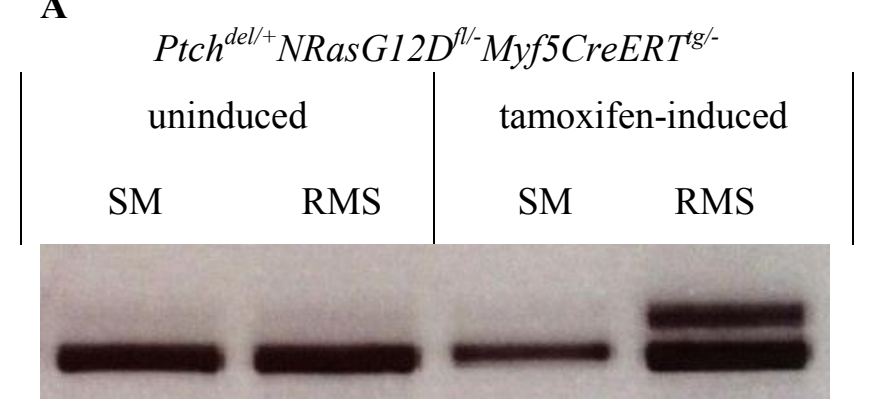

B

Btch ${ }^{\text {dell/ }}$ KRasG $12 D^{f l /}$ Myf5CreERT $T^{g /-}$

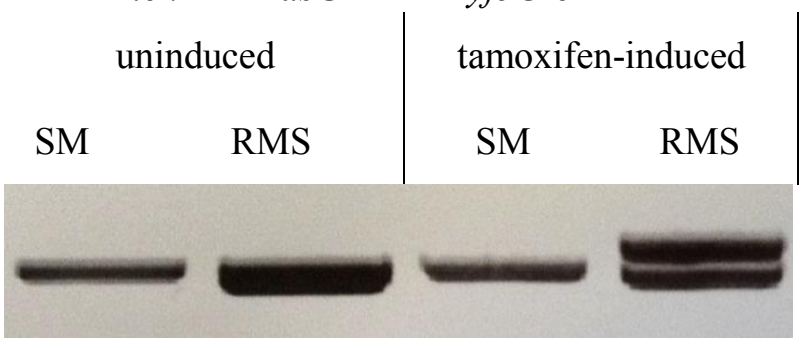

C

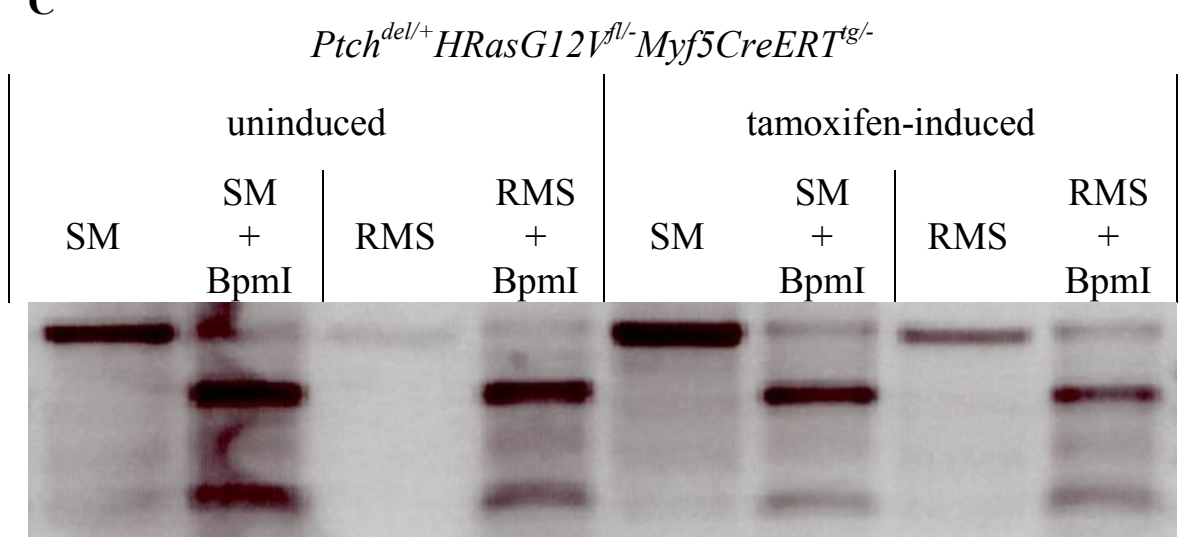

Figure 29: Recombination at the Ras loci in tamoxifen induced oncNRas and oncKRas Ptch $^{\text {del/ } /+}$ oncRas ${ }^{f l /-}$ Myf5CreERT ${ }^{\text {tg/ }}$ mice. The recombination at the Ras loci was analyzed by PCR on gDNA obtained from SM and RMS of uninduced and tamoxifen-induced (A) $\operatorname{Ptch}^{\text {del/ }} N$ Ras G12D $D^{f / /}$ Myf5creERT $T^{g /-}$, (B) Ptch $^{\text {del/ }}$ KRasG12D $D^{f / /} M y f 5 c r e E R T^{g / /}$ mice and (C) on cDNA from SM and RMS undigested and digested with BpmI of uninduced and tamoxifen-induced Ptch ${ }^{\text {del/ }} H$ RasG $12 \mathrm{~V}^{\mathrm{fl} / \mathrm{-}}$ $M y f 5 c r e E R T^{t g /}$ mice. For detailed explanation see text.

In order to get insight into the functionality of the oncRas alleles, we next measured Ras activity was measured in RMS of uninduced and tamoxifen-induced $\operatorname{Ptch}^{\text {del/+ }} \mathrm{NRas} G 12 \mathrm{D}^{\mathrm{fl} / \mathrm{-}}$

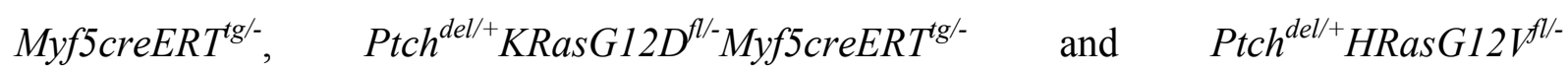
Myf5creERT $T^{\mathrm{tg} / \mathrm{-}}$ mice using the Ras Activation ELISA Assay Kit. As demonstrated in Figure 30 , the Ras signaling activity was increased in RMS of the induced $\operatorname{Ptch}^{\text {del/+ }} N$ Ras $G 12 D^{f l /-}$

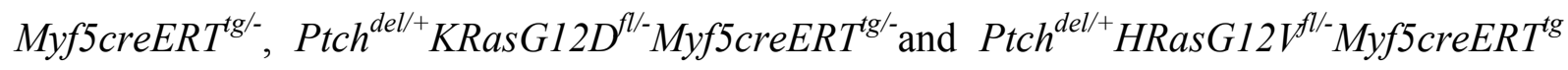
mice compared to none-induced mice. Together these data show that the recombination at the Ras loci after tamoxifen-mediated Cre induction was successful and results in the activation of the Ras pathway in RMS of induced mice. 
A Ptch $^{\text {del/+ }}$ NRasG12D $D^{f l /+}$ Myf 5 Cre $e^{\text {tg/- }}$

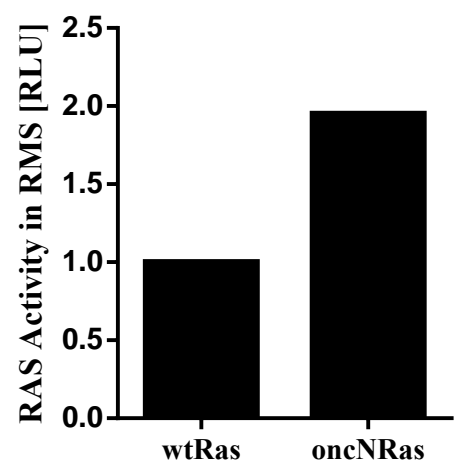

B

Ptch $^{\text {del/+}}$ KRasG $12 D^{f l /+} M y f 5 C r e^{t g /-}$

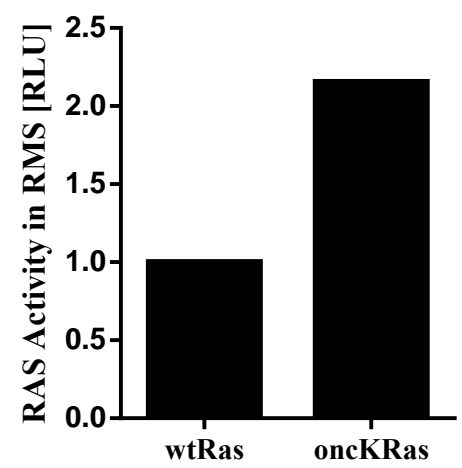

C

Ptch $^{\text {del/ }}$ HRasG12 $V^{f l /+}$ Myf 5 Cre $e^{\text {gg/- }}$

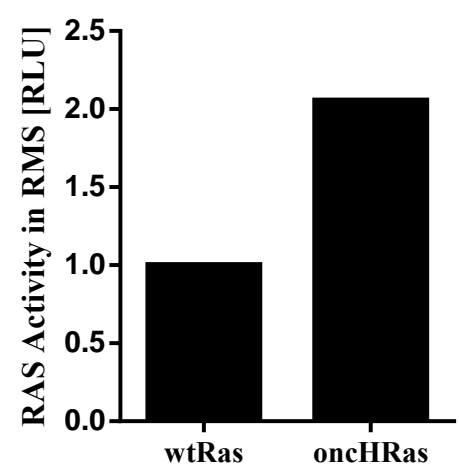

Figure 30: Enhanced Ras activity in RMS from induced Ptch ${ }^{d e / /+}$ oncRas $^{t / /-}$ Myf5creERT $^{t g /-}$ mice. Ras activity analysis using the Ras Activation ELISA ASSAY Kit in protein lysates of RMS from uninduced (wtRas) and tamoxifen-induced (oncRas) (A) Ptch ${ }^{\text {del/ }} N R a s G 12 D^{f / /} M y f 5 c r e E R T^{\text {tg/ }}$ mice $\quad$ (B)

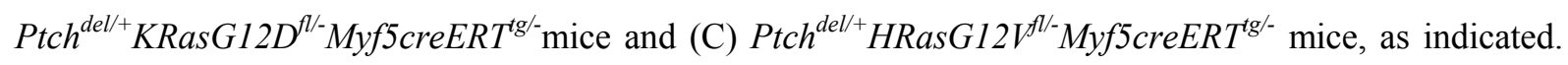
The Ras activity in wtRas RMS was set to 1 .

In a next setting of preparatory experiments, the influence of tamoxifen on the RMS growth was analyzed. For this purpose $P t c h^{\text {del/+ }}$ mice on exactly the same background as Ptch $^{\text {del/+ }}$ oncRas Myf5creERT $T^{\text {tg/ }}$ mice were used. As shown in Table 18 and Figure 31, the injection of $1 \mathrm{mg}$ tamoxifen on 5 consecutive days slightly increases but does not significantly change the RMS free survival of the animals. It also does not change the tumor incidence or RMS multiplicity.

Table 18: Influence of Tamoxifen on RMS development of $\operatorname{Ptch}^{\text {del/+ }}$ mice

\begin{tabular}{lcccccc}
\hline Genotype & $\begin{array}{c}\text { Status of } \\
\text { tamoxifen } \\
\text { induction }\end{array}$ & $\mathbf{N}$ & $\begin{array}{c}\text { Mice with } \\
\text { RMS }\end{array}$ & $\begin{array}{c}\text { Mice with } \\
\text { multiple } \\
\text { RMS }\end{array}$ & $\begin{array}{c}\text { RMS } \\
\text { number } \\
\text { per mouse } \\
(\text { mean } \pm \text { SEM) }\end{array}$ & $\begin{array}{c}\text { Median latency } \\
\text { time of RMS } \\
\text { (days) }\end{array}$ \\
\hline Ptch $^{\text {del/ } /+}$ & uninjected & 30 & $\begin{array}{c}17 \\
(60.7 \%)\end{array}$ & $9(52.9 \%)$ & $1.650 \pm 0.187$ & 60 \\
Ptch $^{\text {del/ } /+}$ & $\begin{array}{c}14 \\
\text { tamoxifen- } \\
\text { injected }\end{array}$ & 29 & $5(35.7 \%)$ & $1.429 \pm 0.173$ & 118 \\
\hline
\end{tabular}


A

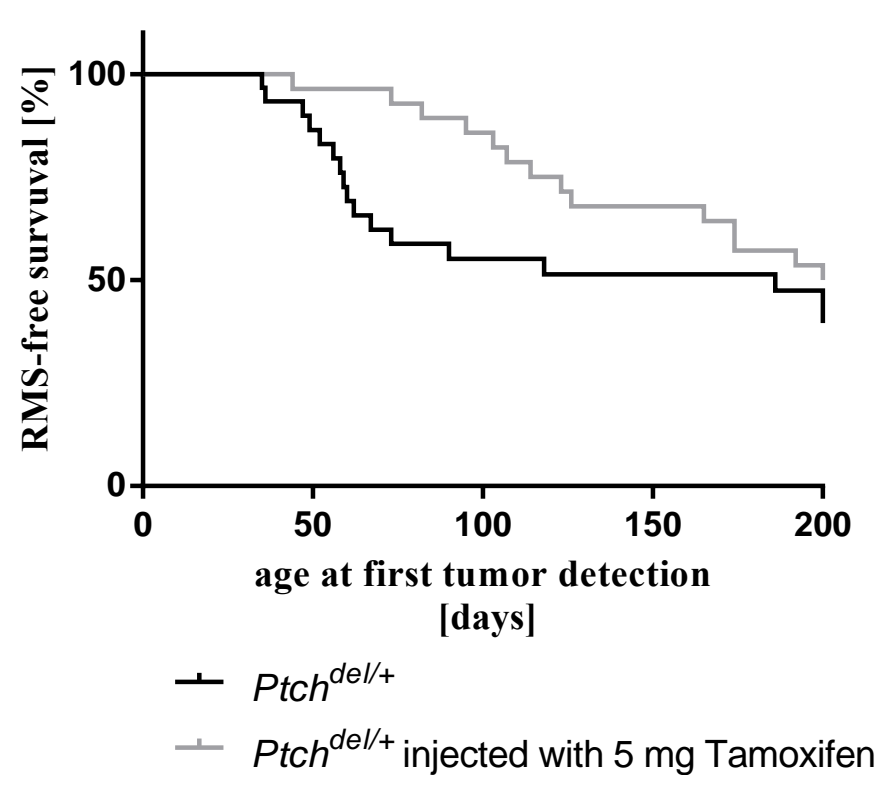

B

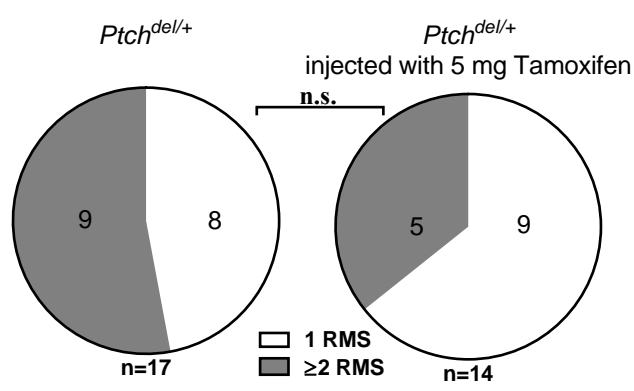

Figure 31: No significant effect of tamoxifen on RMS incidence, multiplicity and latency time. (A) Kaplan Mayer Curve showing the RMS free survival of $\mathrm{Ptch}^{\mathrm{del/+}}$ mice (black) and $\mathrm{Ptch}^{\mathrm{del} / \mathrm{+}}$ mice injected with $5 \mathrm{mg}$ tamoxifen (grey). Every event represents the detection of the first RMS in a mouse. (B) Graph shows the tumor multiplicity as RMS/animal of $\mathrm{Ptch}^{\mathrm{del} /+}$ mice and $\mathrm{Ptch}^{\mathrm{del} /+}$ mice injected with $5 \mathrm{mg}$ tamoxifen. Statistical significance of the RMS-free survival was tested by log-rank test $(P=0.1955)$, of the latency time by Gehan-Breslow-Wilcoxon test $(P=0.078)$ and of the multiplicity by Chi-squared test $(P=0.1687)$.

\subsubsection{Characterization of RMS after conditional expression of oncNRas in Ptch mutant mice}

To analyze the effects of the expression of oncNRas on the growth of Hh-associated RMS, 65 Ptch $^{\text {del// }}$ NRasG $12 D^{f / /}$ Myf5CreERT $T^{\text {tg/- }}$ mice were randomized in two groups and treated with tamoxifen or left untreated as described above.

\subsubsection{1. oncNRas increases the expression of Gli transcription factors and downregulates expression of early and late muscle markers in RMS of Ptch mutant mice}

As mentioned above expression of oncRAS in human RMS cell lines can influence $\mathrm{HH}$ signaling activity. In order to evaluate whether this was also true for RMS of Ptch mutant mice the expression levels of Glil-3 was measured in skeletal muscle that was used as a 
reference tissue and in RMS from tamoxifen-induced and uninduced $\operatorname{Ptch}^{\mathrm{del} / \mathrm{+}} \mathrm{NRas} G 12 \mathrm{D}^{\mathrm{fl} / \mathrm{-}}$

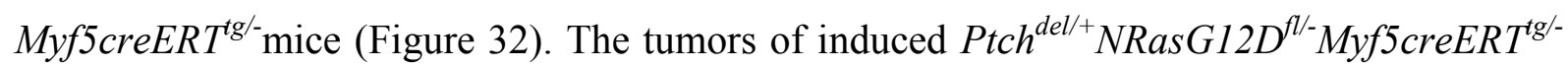
mice $(\mathrm{n}=6)$ show a significantly increased expression of the Hh pathway components Gli2 and Gli3 while the expression of Gli1 seems to be unchanged.

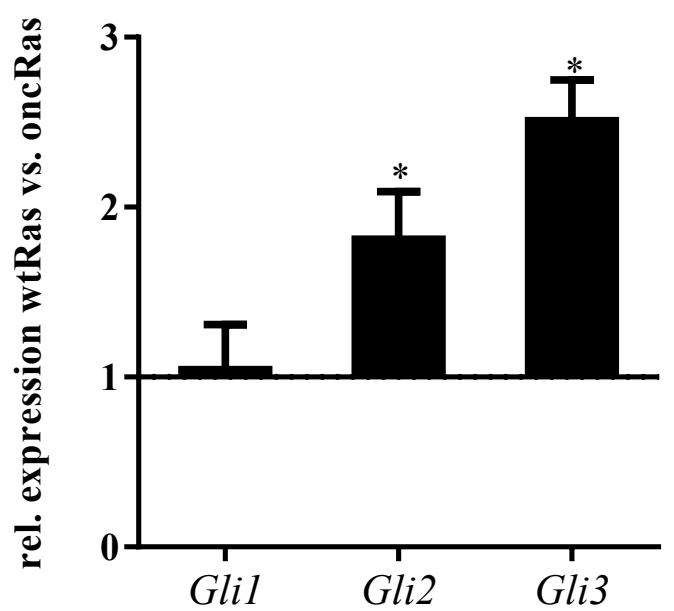

Figure 32: Increased expression of Gli2 and Gli3 by oncNRas in Ptch mutant RMS. Quantification of the Gli1, Gli2 and Gli3 expression levels measured by qRT-PCR analyses on cDNA from RMS of not induced (expression levels were set to 1) and induced Ptch $^{\text {del/ }}$ NRasG12D $D^{f l /} M y f 5 \mathrm{creERT}^{\mathrm{tg} /-}$ mice. For this purpose gene expression was measured in both RMS and skeletal muscle. Then all expression levels were normalized to $18 S$ rRNA levels. Next the data was normalized to expression in normal skeletal muscle of the same animal. Then all data was pooled and the plots finally show the relation of expression levels of oncNRas RMS to wtRas RMS that was set to 1 . All data are displayed as mean \pm SEM. Statistical significance was tested by Mann-Whitney test. $* P<0.05$

Due to the fact that active Ras signaling can influence Raf/Mek/Erk and PI3K/Akt signaling, the phosphorylation status of Erk and Akt were examined by Western blot analysis (see Chapter 4.16.3). As shown in Figure 33 the expression of oncNRas in Ptch mutant RMS does not obviously change phosphorylation levels of Erk and Akt. 


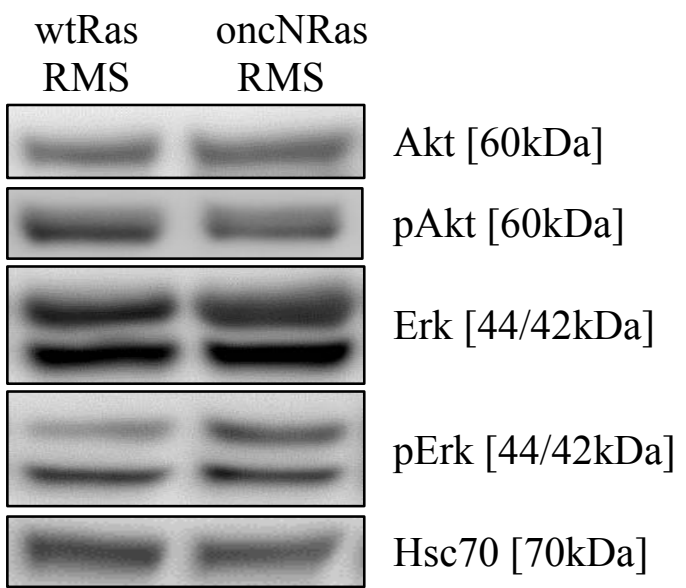

Figure 33: No obvious phosphorylation changes in oncNRas-expressing Ptch mutant RMS. Western blot analyses of Akt, pAkt, Erk, pErk in protein lysates from RMS isolated from uninduced (wtRas) and

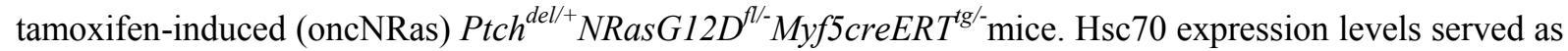
loading control.

Since Ras signaling also influences differentiation processes, the expression of the myogenesis proliferation and determination markers $M y o D$ and $M y f 5$ and of the differentiation marker Myogenin was measured by qRT PCR in skeletal muscle and RMS of wtRas $(n=6)$ and oncNRas $(n=6)$ mice. As illustrated in Figure 34, the expression of the markers is decreased in RMS that express oncogenic NRas compared to control. Although the decrease is not significant that data indicate that oncogenic NRas may inhibit the expression of muscle differentiation in RMS, at least in this mouse model.

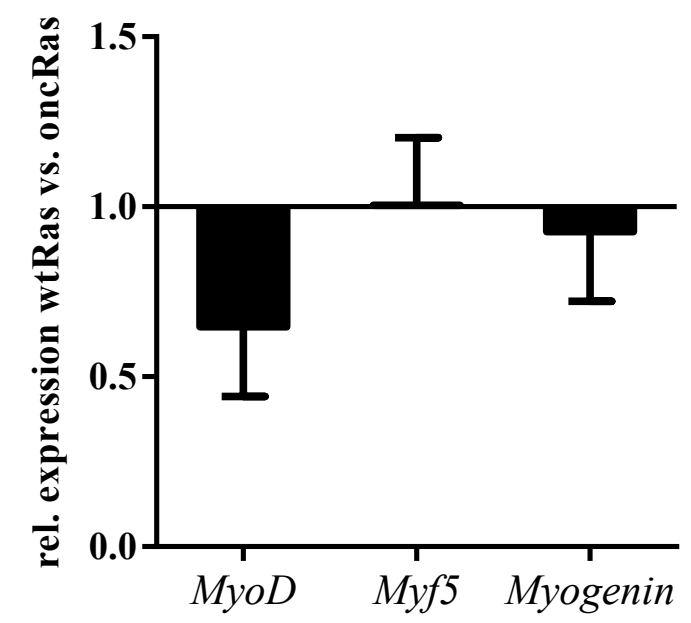

Figure 34: Decreased expression of MyoD and Myogenin in oncNRas-expressing Ptch mutant RMS.

Quantification of MyoD, Myf5 and Myogenin expression levels measured by qRT-PCR analyses on cDNA from RMS and skeletal muscle of uninduced and tamoxifen-induced Ptch $^{\text {del// }} N R a s G 12 D^{f l /} M y f 5 \mathrm{creERT}^{\mathrm{tg} /-}$ mice. Normalization procedure is given in Figure 32. The All data are displayed as mean \pm SEM. Statistical significance was tested by using Mann-Whitney test. 


\subsubsection{2. oncNRas decreases multiplicity of RMS in Ptch mutant mice}

To investigate the effects of oncNras on the tumor incidence, latency time and RMS multiplicity, the mice were monitored weekly (see chapter 4.17.4) until an age of 200 days, if possible. As shown in Table 19 and Figure 35, the analysis of $\operatorname{Ptch}^{\text {del/ }} N$ RasG12D $D^{f l-}$ Myf5CreERT ${ }^{\text {tg/- }}$ mice (wtRas $\mathrm{n}=32$; oncNRas $\mathrm{n}=33$ ) shows no significant differences in tumor incidence $(62.5 \%$ vs $60.6 \%)(P=0.835$ for palpable RMS by log-rank test $)$ or tumor-latency time (77.5 days vs 93 days) ( $P=0.8449$ for RMS by Gehan-Breslow-Wilcoxon test) between tamoxifen-induced mice compared to uninduced ones (Figure 35A). Thus, the NRas mutation did not alter the RMS-free survival. However, several mice developed more than one RMS, which were either detected during life or upon autopsy. Most interestingly, in mice with the NRas mutation the average number of tumors per mouse $(1.850 \pm 0.196$ vs $1.364 \pm 0.140)$ and the occurrence of multiple tumors (60\% vs $30 \%$ ) was significantly decreased compared to the wtRas controls $(P=0.0283)$ (Table 19). (Figure 35B). Together, these data suggest that oncogenic NRas in the Ptch mouse model for RMS inhibits tumor initiation and probably also progression of the tumors.

Table 19: Influence of oncNRas on RMS development of $\operatorname{Ptch}^{\text {del/ }} N$ RasG $12 D^{f l /-}$ Myf5CreERT ${ }^{t g /-}$ mice

\begin{tabular}{|c|c|c|c|c|c|c|}
\hline Genotype & $\begin{array}{l}\text { Status of } \\
\text { tamoxifen } \\
\text { induction }\end{array}$ & n & $\begin{array}{l}\text { Mice } \\
\text { with } \\
\text { RMS }\end{array}$ & $\begin{array}{l}\text { Mice with } \\
\text { multiple } \\
\text { RMS }\end{array}$ & $\begin{array}{c}\text { RMS } \\
\text { number } \\
\text { per mouse } \\
(\text { mean } \pm \text { SEM })\end{array}$ & $\begin{array}{c}\text { Median } \\
\text { latency } \\
\text { time of } \\
\text { RMS (days) }\end{array}$ \\
\hline $\begin{array}{l}\text { Ptch }^{\text {del/t+} N R a s G 12 D^{f l /-}} \\
\text { Myf5CreERT }\end{array}$ & uninduced & 32 & $\begin{array}{c}20 \\
(62.5 \%)\end{array}$ & $12(60 \%)$ & $1.850 \pm 0.196$ & 77.5 \\
\hline $\begin{array}{l}\text { Ptch }^{\text {del/+ }} \text { NRasG12D } \\
\text { Myf5CreERT }{ }^{f / /-}\end{array}$ & $\begin{array}{l}\text { tamoxifen- } \\
\text { induced }\end{array}$ & 33 & $\begin{array}{c}20 \\
(60.6 \%)\end{array}$ & $6(30 \%)$ & $1.364 \pm 0.140$ & 93 \\
\hline
\end{tabular}


A

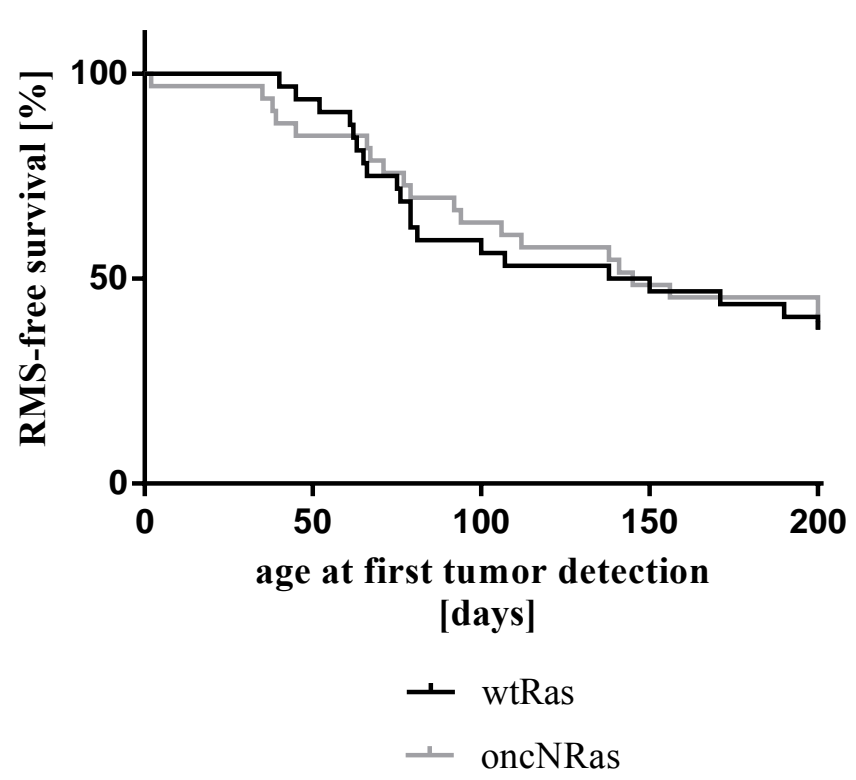

B

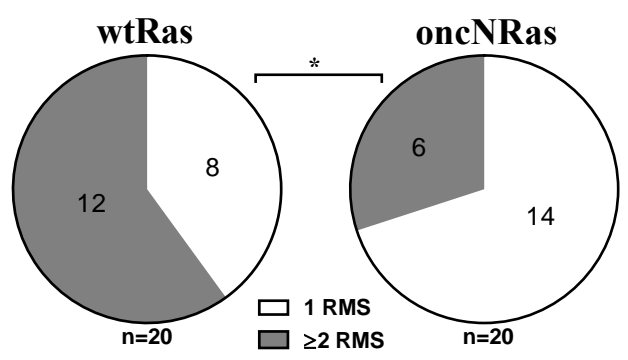

Figure 35: oncNRas decreases multiplicity of RMS of Ptch mutant mice. (A) Kaplan Mayer Curve showing the RMS free survival of wtRas (black) and oncNRas (grey) Ptch ${ }^{\text {del/ }} N R a s G 12 D^{f / /}$ Myf5CreERT $T^{t g /-}$ mice. Every event represents the detection of the first RMS in a mouse. (B) Graph shows the tumor multiplicity as RMS/animal of wtRas and oncNRas $\operatorname{Ptch}^{\text {del/ }} N$ Ras G12 $D^{f l /} M y f 5 C r e E R T^{t g /-}$ mice. Statistical significance of the RMS-free survival was tested by log-rank test $(P=0.8356)$, of the latency time by Gehan-Breslow-Wilcoxon test $(P=0.8449)$ and of the multiplicity by Chi-squared test $(P=0.0283)$.

\subsubsection{3. oncNRas decreases the proliferation rate of RMS of Ptch mutant mice}

In order to investigate the impact of oncNRas on proliferative capacity of RMS of Ptch mutant mice immunohistochemically stainings of RMS were performed with the Ki67 antibody. Ki67 is a nuclear protein required for proliferation. The tumors derived from wtRas $(\mathrm{n}=8)$ and oncNRas $(\mathrm{n}=7)$ Ptch $^{\text {del//+}} N \operatorname{Ras} G 12 D^{f l /-}$ Myf5CreERT $T^{t g /-}$ mice were stained and Ki67 positive and negative nuclei were calculated (Figure 36 ). 
A

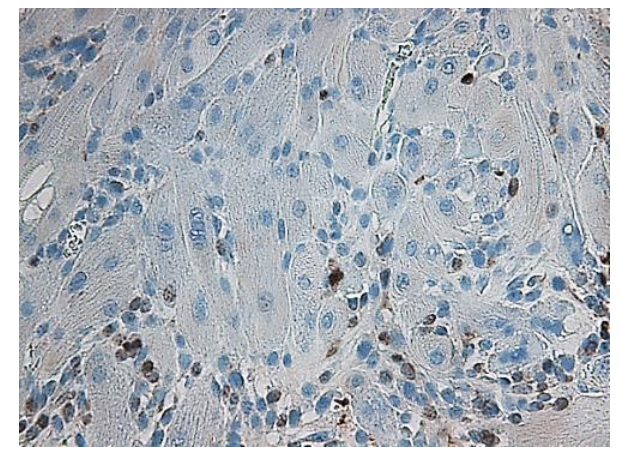

B

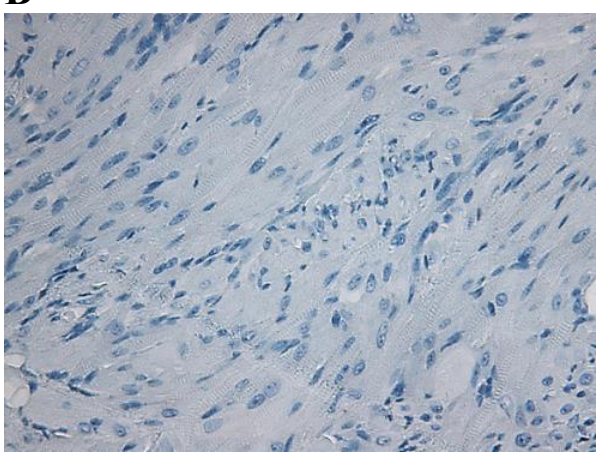

C

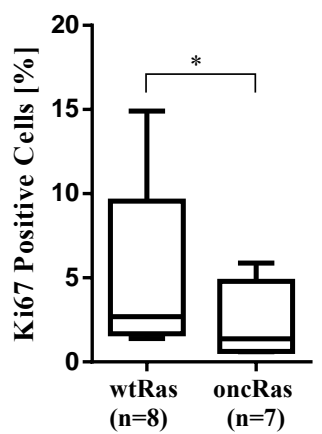

Figure 36: Decreased proliferation of oncNRas expressing RMS of Ptch mutant mice. Ki-67 staining of tumors derived from Ptch $^{\text {del/ }} N R a s G 12 D^{f l /}$ Myf5CreERT $T^{\text {tg/- }}$ mice expressing (A) wtRas (20x magnification) or (B) oncNRas (20x magnification). (C) Percentage of Ki67 positive nuclei counted on paraffin section of wtRas and oncNRas-expressing RMS derived from Ptch mutant mice. 6 pictures per section per RMS were analyzed. More than 1000 cells were counted. All data are displayed as mean \pm SEM. The cells were counted using image processing software FIJI as described in the Material and Methods section. Statistical significance was analyzed by Mann-Whitney Test. ${ }^{*} P<0.05$

The results revealed that oncNRas RMS significantly reduced the number of Ki67 positive cells compared to RMS from wtRas animals $(2.249 \% \pm 0.8144 \%$ vs $5.311 \% \pm 1.768 \%)$. These results indicate that oncogenic NRas reduces the proliferative capacity and thus the progression of Ptch deficient RMS.

The histological characterization by microscopy was done in collaboration with Dr. Walter Schulz-Schäfer, Department of Neuropathology at the University of Göttingen. As revealed by H\&E staining (see chapter 4.16.5) oncNRas expression does not change the histology of RMS of Ptch mutant mice (Figure 37). 
A

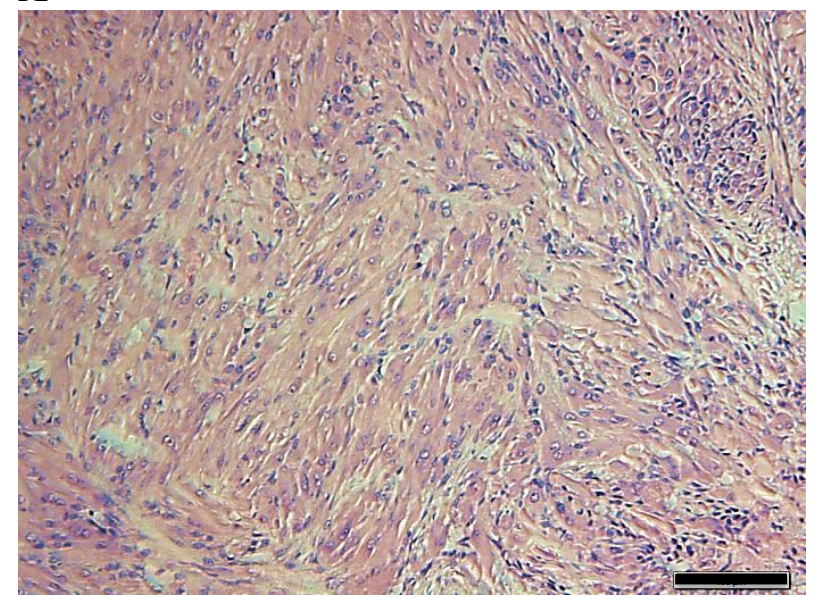

C

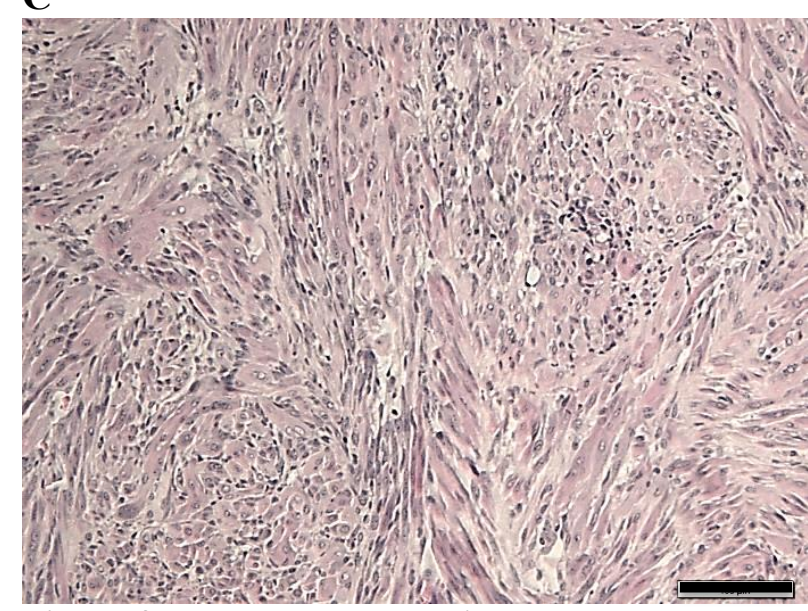

B

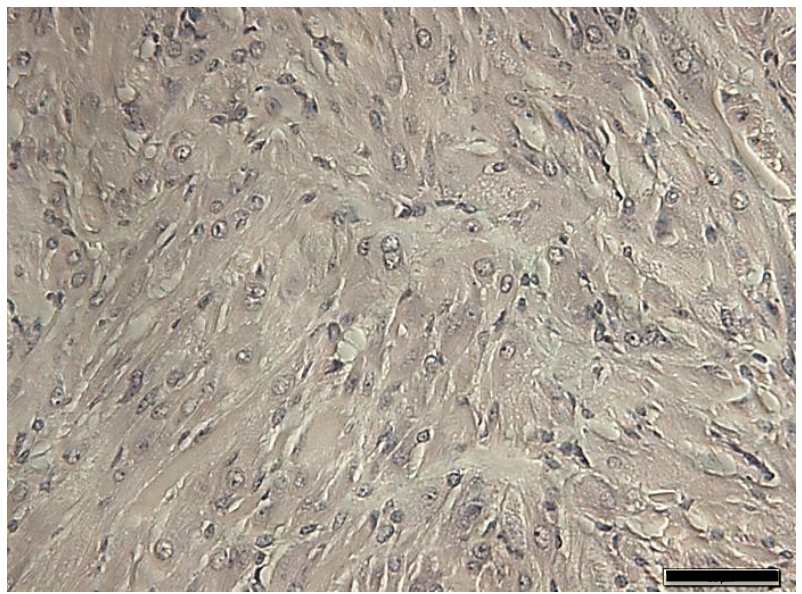

D

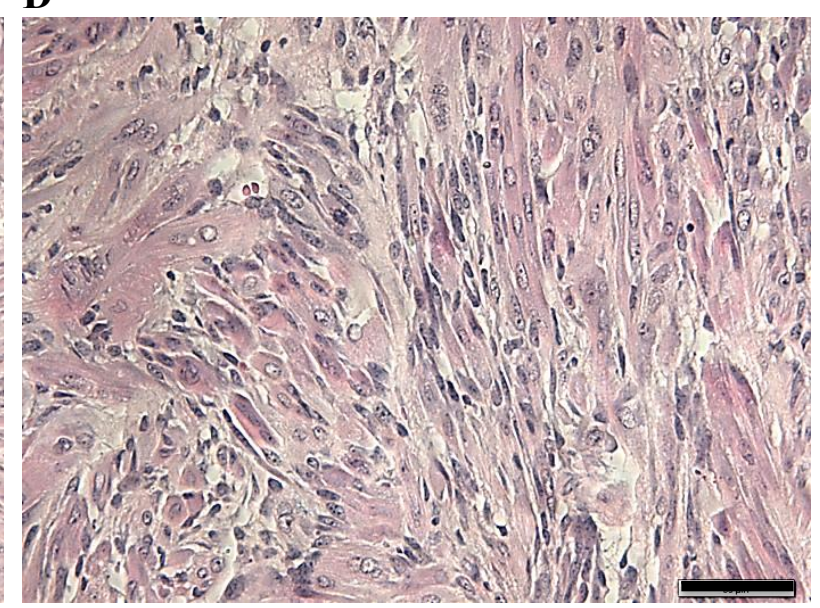

Figure 37: oncNRas expression does not change the histology of Ptch mutant RMS. H\&E stainings of RMS derived from Ptch $^{\text {del/ }}$ NRasG12D ${ }^{f l /}$ Myf5CreERT $T^{\mathrm{tg} /}$ mice expressing wtRas are shown in (A) and (B). (A) shows 10x magnification; scale bar $=100 \mu \mathrm{m}$ and (B) a 20x magnification; scale bar $=50 \mu \mathrm{m}$. (C) and (D) show RMS expressing oncNRas. (C) shows a 10x magnification, scale bar $=100 \mu \mathrm{m}$ and (D) a $20 \mathrm{x}$ magnification; scale bar $=50 \mu \mathrm{m}$.

\subsubsection{Characterization of RMS after conditional expression of oncKRas in Ptch mutant mice}

To analyze the effects of the expression of oncKRas on the growth of Hh-associated RMS 65 Ptch $^{\text {del/ }}$ KRasG12 $D^{f l /-}$ Myf5CreERT $T^{t g /-}$ mice were randomized in two groups. 


\subsubsection{1. oncKras decreases the expression of Gli transcription factors, increases pErk and pAkt levels and enhances the expression of early muscle differentiation markers in RMS of Ptch mutant mice}

As already seen in the study of $\operatorname{Ptch}^{\mathrm{del} / \mathrm{H}} \mathrm{NR}$ as $G 12 D^{f l /} \mathrm{Myf} 5 \mathrm{creERT} T^{\mathrm{tg} /}$-mice oncRAS can influence HH signaling activity. In order to evaluate whether this was also true for RMS of Ptch $^{\text {del// }}$ KRasG $12 D^{f l /-}$ Myf5creERT ${ }^{t g /-}$ mice the expression of Gli1-3 was measured in the reference tissue skeletal muscle and RMS from tamoxifen-induced $(n=5)$ and uninduced $(n=2)$ mice (Figure 38). Preliminary expression analysis shows that the expression of all three Glis

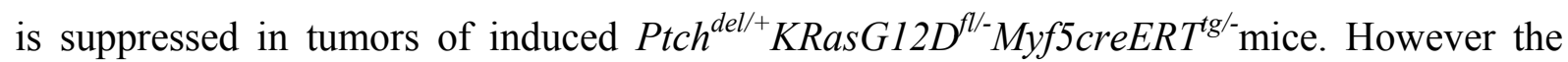
suppression was not significant.

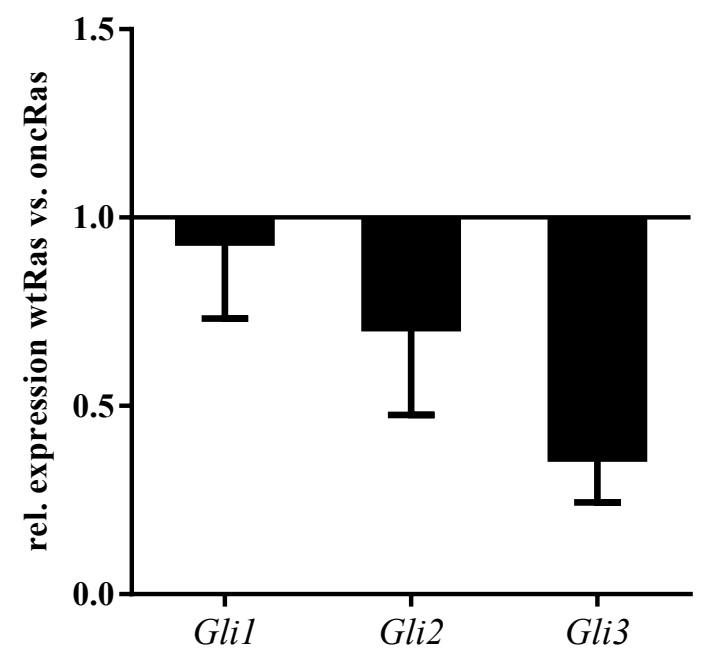

Figure 38: oncKRas decreases the expression of Glis in RMS of Ptch mutant mice. Quantification of the Gli1, Gli2 and Gli3 expression levels measured by qRT-PCR analyses on cDNA from RMS and

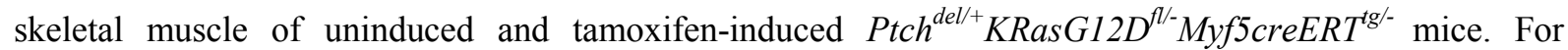
normalization of the data see Figure 32. All data are displayed as mean \pm SEM. Statistical significance was tested by using Mann-Whitney test.

Furthermore a western blot analysis from uninduced and tamoxifen-induced Ptch $^{\text {del// }}$ KRasG12D $D^{f / /}$ Myf5creERT $T^{t g /-}$ mice was performed (see Chapter 4.16.3). To see whether active KRas signaling can influence Raf/Mek/Erk and PI3K/Akt signaling the phosphorylation status of Erk and Akt was examined. Figure 39 shows that the expression of oncKRas in Ptch mutant RMS results in increased phosphorylation of Erk and Akt, which 
indicates that active KRas signaling in oncRas $\operatorname{Ptch}^{\text {del//+}}$ KRas $G 12 D^{f l /} M y f 5 \mathrm{creERT}^{\mathrm{tg} / \text { mice }}$ results in the activation of the Raf/Mek/Erk and PI3K/Akt pathways.

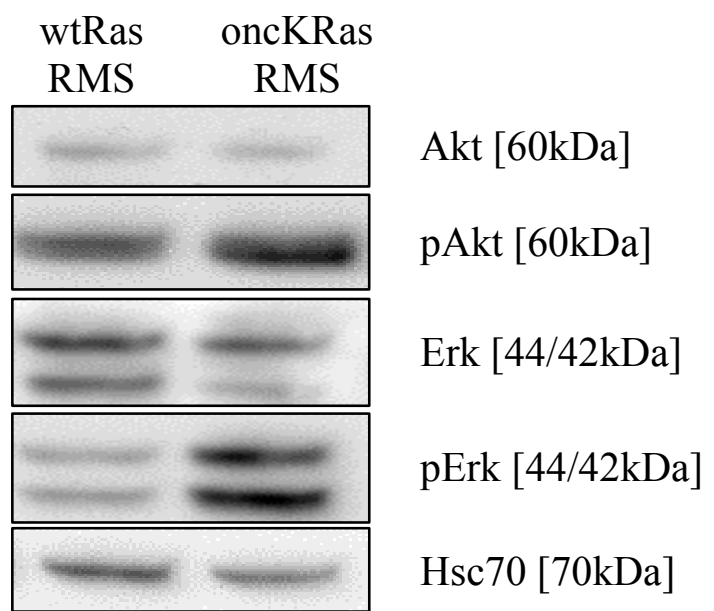

Figure 39: oncKRas increases pErk and pAkt levels in RMS of Ptch mutant mice. Western blot analyses of Akt, pAkt, Erk, pErk in protein lysates from RMS isolated from wtRas and oncKRas Ptch $^{\text {del/ }}$ KRasG12D ${ }^{f l /-}$ Myf5 creERT $^{\text {tg/- }}$ mice. Hsc70 expression levels served as loading control.

Next the expression of muscle differentiation markers were measured by qRT PCR in skeletal

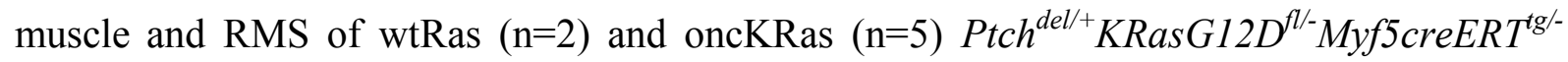
mice. The preliminary data illustrated in Figure 40 show an increased expression of the early differentiation markes $M y o D$ and $M y f 5$ and a suppressed expression of the late differentiation marker Myogenin in RMS expressing oncogenic KRas compared to the control. Although the modulations are not significant (probably due to the small sample size) the data indicate that oncogenic KRas may cause a more undifferentiated RMS phenotype i.e. it causes expression of myogenesis proliferation and determination markers and concomitantly block late muscle differentiation. 


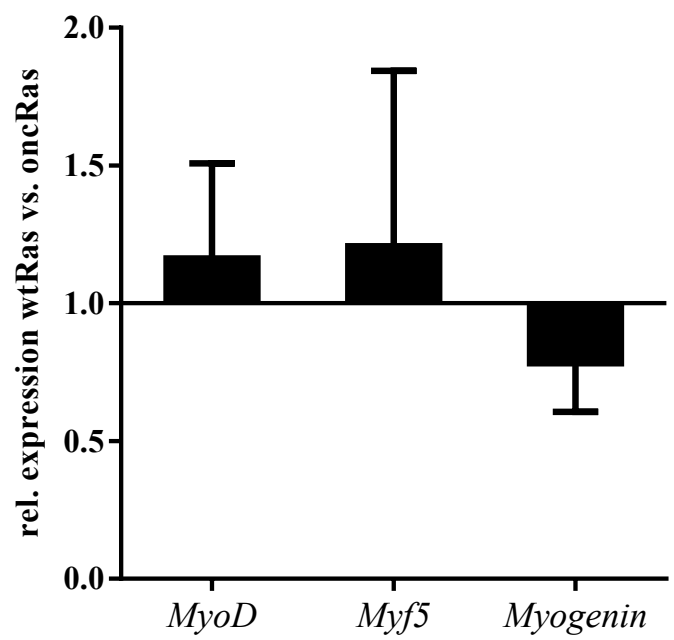

Figure 40: Slightly increased expression of the myogenesis proliferation and determination markers and decreased muscle differentiation marker in oncRas RMS of Ptch mutant mice. Quantification of the MyoD, Myf5 and Myogenin expression levels measured by qRT-PCR analyses on cDNA from RMS and skeletal muscle of uninduced and tamoxifen-induced Ptch $^{\text {del/ }} K_{R a s G 12 D^{f l /} M y f 5} \mathrm{creERT}^{\mathrm{tg} / \text { mice. }}$ Normalization was done as described in Figure legend 32. All data are displayed as mean \pm SEM. Statistical significance was tested by using Mann-Whitney test.

\subsubsection{2. oncKRas increases incidence and shortens latency time of RMS in Ptch mutant mice}

In order to investigate if the above mentioned modulation of oncKRas has an influence on the tumor incidence, latency time RMS multiplicity, the mice were monitored weekly (see chapter 4.17.4) for up to 200 days. As illustrated in Table 20 and Figure 41, the analysis of Ptch $^{\text {del// }}$ KRasG $12 D^{f / /}$ Myf5CreERT $T^{\text {tg/- }}$ mice shows significant differences in tumor development. Thus, in mice expressing oncogenic KRas the tumor incidence is significantly increased when compared to the control ( $82.2 \%$ vs $49.3 \%$, respectively) ( $P=0.0013$ for RMS by log-rank test). In addition the tumor-latency time is significantly decreased (79.7 days vs 102.8 days, respectively) ( $P=0.0018$ for RMS by Gehan-Breslow-Wilcoxon test). However the oncKRas does not influence the tumor multiplicity. 
Table 20: Influence of oncKRas on RMS development of $\operatorname{Ptch}^{\text {del/ }} K R a s G 12 D^{f l /-}$ Myf5CreERT $T^{\text {tg- }}$

\begin{tabular}{|c|c|c|c|c|c|c|}
\hline Genotype & $\begin{array}{l}\text { Status of } \\
\text { tamoxifen } \\
\text { induction }\end{array}$ & $\mathbf{n}$ & $\begin{array}{l}\text { Mice } \\
\text { with } \\
\text { RMS }\end{array}$ & $\begin{array}{c}\text { Mice with } \\
\text { multiple } \\
\text { RMS }\end{array}$ & $\begin{array}{c}\text { RMS } \\
\text { number } \\
\text { per mouse } \\
(\text { mean } \pm \text { SEM })\end{array}$ & $\begin{array}{c}\text { Median } \\
\text { Latency } \\
\text { time of } \\
\text { RMS (days) }\end{array}$ \\
\hline $\begin{array}{l}\text { Ptch }^{\text {del/t }} \text {KRRasG12D }^{\text {fl/- }} \\
\text { Mvf5CreERT }\end{array}$ & uninduced & 28 & $\begin{array}{c}13 \\
(49.3 \%)\end{array}$ & $6(46.2 \%)$ & $1.615 \pm 0.241$ & 103 \\
\hline $\begin{array}{l}\text { Ptch }^{\text {del/ } /+} \text { KRasG }_{12} D^{f l /-} \\
\text { Myf5CreERT }^{\text {tg/- }}\end{array}$ & $\begin{array}{l}\text { tamoxifen- } \\
\text { induced }\end{array}$ & 30 & $\begin{array}{c}24 \\
(82.2 \%)\end{array}$ & $\begin{array}{c}13 \\
(54.2 \%)\end{array}$ & $1.583 \pm 0.119$ & 79 \\
\hline
\end{tabular}

A

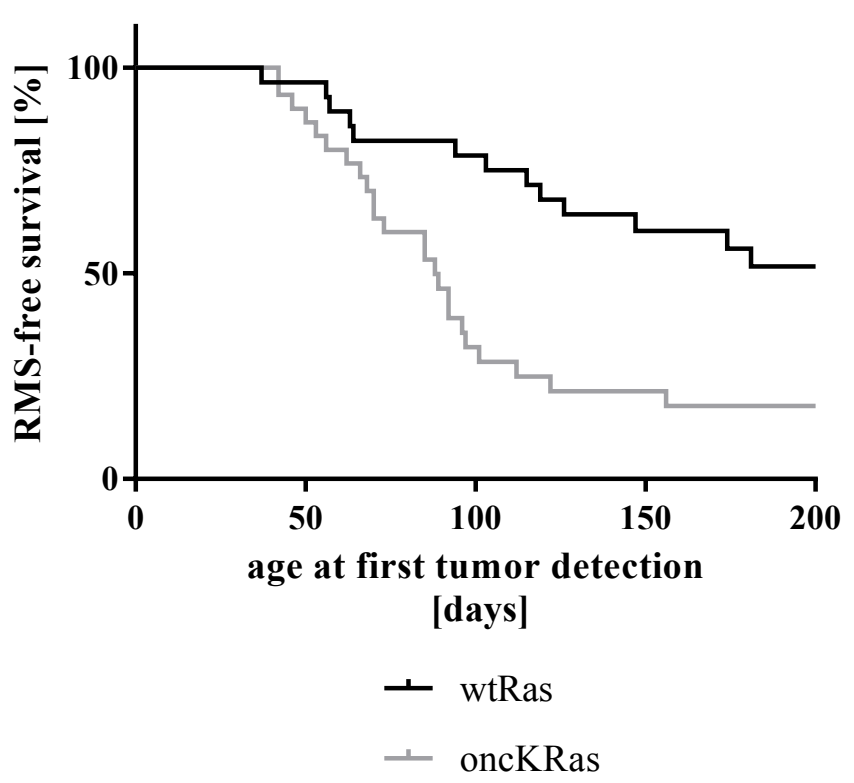

B

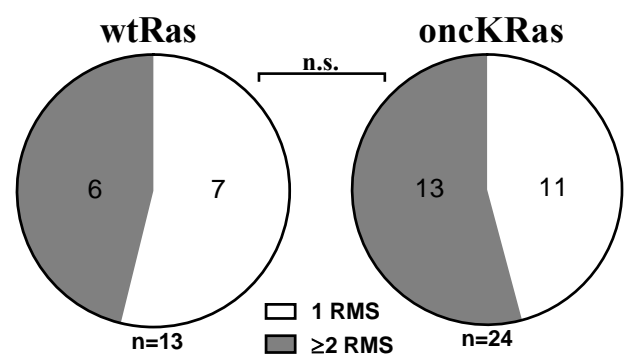

Figure 41: oncKRas significantly increases tumor incidence and shortens latency time. (A) Kaplan Mayer Curve with the RMS free survival of wtRas (black) and oncKRas (grey) Ptch $^{\text {del/ }} K R a s G 12 D^{f l /-}$ Myf5CreERT $T^{\mathrm{tg} /}$ mice. Every event represents the detection of the first RMS in a mouse. (B) Graph shows tumor multiplicity as RMS/animal in wtRas and oncKRas Ptch $^{\text {del/ }}{ }^{2}$ RasG $12 D^{f l /} M y f 5 C r e E R T^{t g /-}$ mice. Statistical significance of the RMS-free survival was tested by log-rank test $(P=0.0013)$, of the latency time by Gehan-Breslow-Wilcoxon test $(P=0.0018)$ and of the multiplicity by Chi-squared test $(P=$ $0.3208)$.

\subsubsection{3. oncKras does not change the proliferation rate or morphology of RMS of Ptch mutant mice}

In order to investigate if the decreased latency time and the increased tumor incidence in oncKRas expressing Ptch mutant mice are caused by changes in the proliferative capacity, RMS isolated from wtRas (n=7) and oncKRas (n=9) $\operatorname{Ptch}^{\text {del/ }}$ KRasG12D $D^{f / /}$ Myf5CreERT $T^{\text {tg/- }}$ mice were stained with the Ki-67 antibody and the Ki-67 positive and negative nuclei were 
calculated (Figure 36). The results show that oncKRas does not significantly increase the number of Ki67 positive nuclei compared to RMS from wtRas animal $(4.21 \% \pm 2.252 \%$ vs $3.782 \% \pm 1.188 \%$ ). This indicates that oncogenic KRas signaling does not or only moderately increase the proliferative capacity of Ptch deficient RMS.
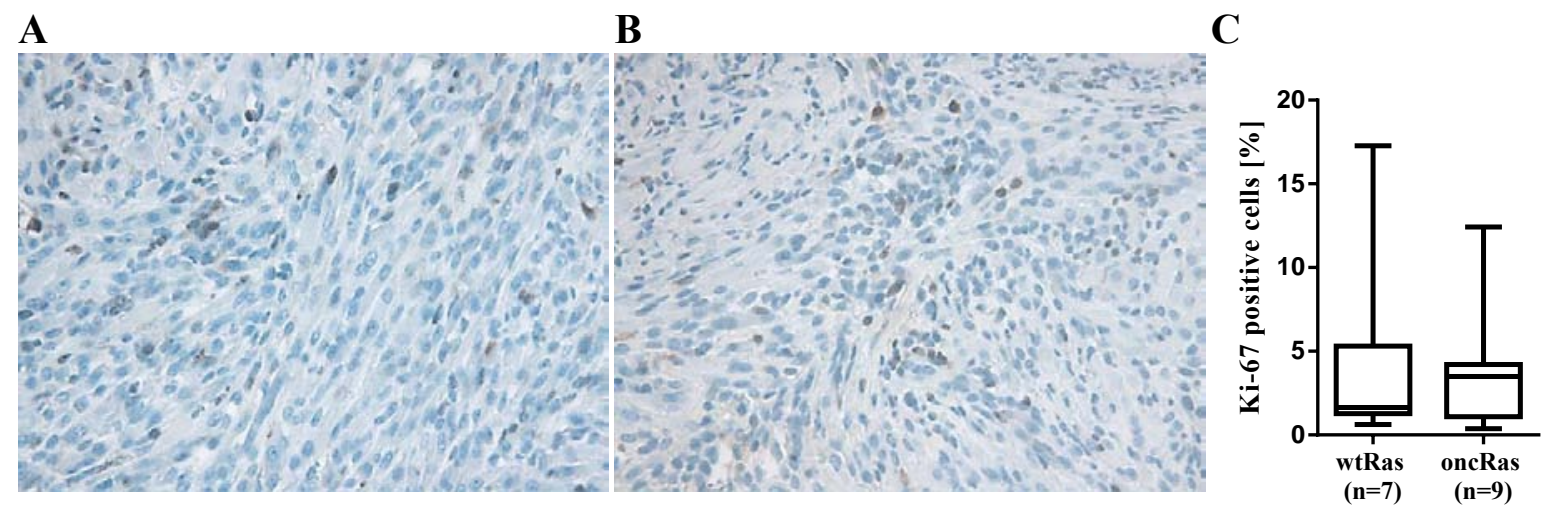

Figure 42: oncKRas does not significantly increase the proliferation rate of RMS of Ptch mutant mice. Ki-67 stainings of tumors derived from Ptch $^{\text {del/ }}{ }^{2}$ Ras $G 12 D^{f / /}$ Myf5CreERT ${ }^{\mathrm{tg} /}$ - mice expressing (A) wtRas (20x magnification) or (B) oncKRas (20x magnification). (C) The plot show the percentage of Ki67 positive nuclei per paraffin section of wtRas and oncRas mice. All data are displayed as mean \pm SEM. The cells were counted using image processing software FIJI. 3 (6 pictures per section per RMS were analyzed. More than 1000 cells were counted). Statistical significance was analyzed using Mann-Whitney test.

As revealed by $\mathrm{H} \& \mathrm{E}$ staining (see chapter 4.16.5) oncKRas expression also does not obviously change the histology of RMS of Ptch mutant mice (Figure 43). 
A

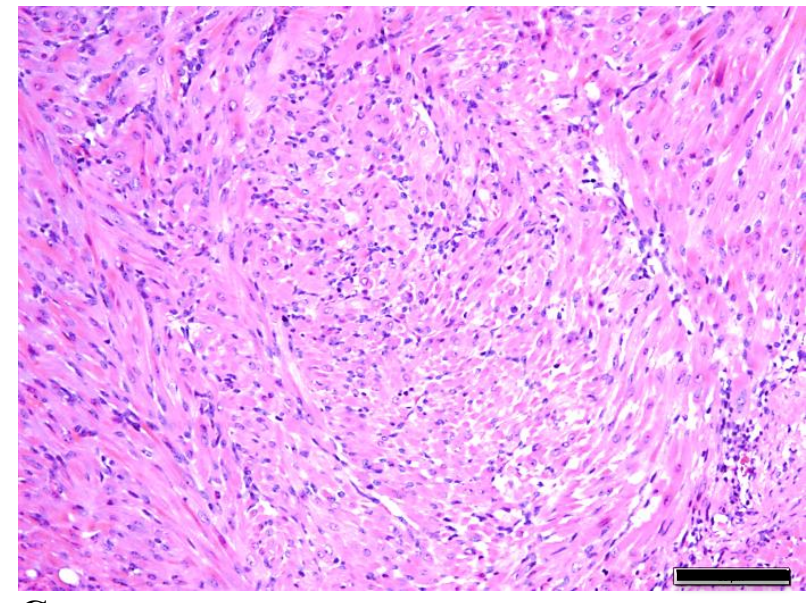

C

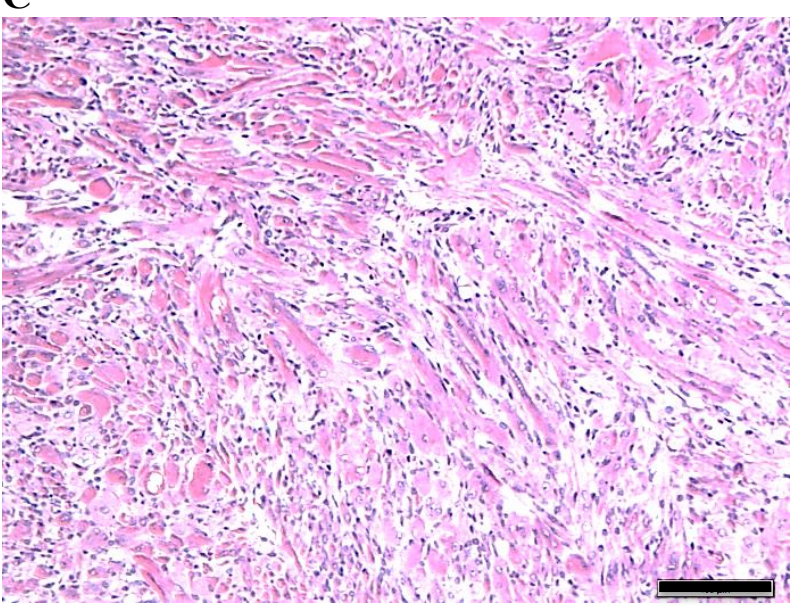

B

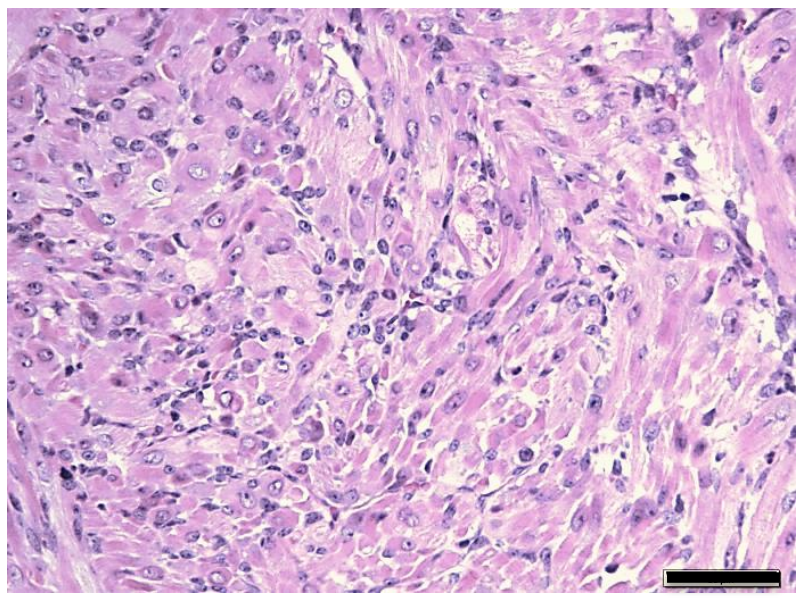

D

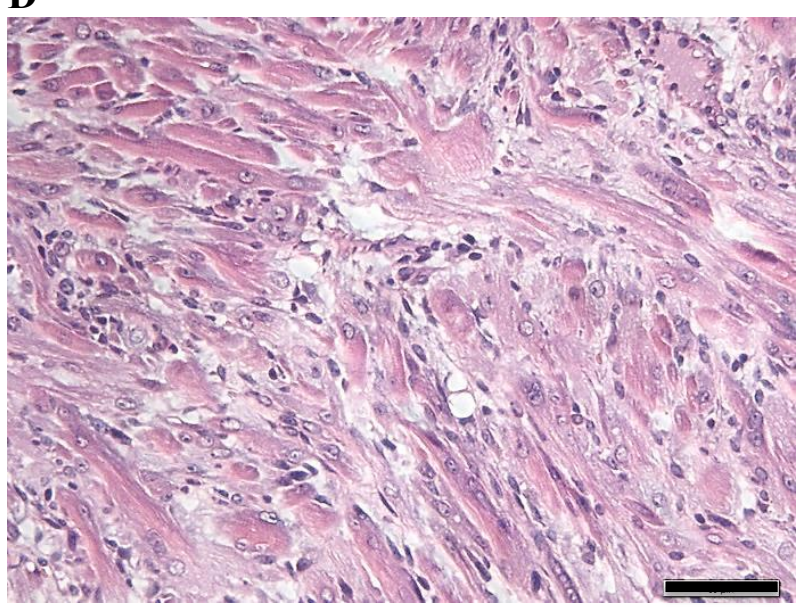

Figure 43: No differences in the tissue structure of RMS with and without active KRas signaling. H\&E stainings of tumors derived from Ptch ${ }^{\text {del/ }}$ KRasG12D $D^{f / /}$ Myf 5 CreERT $T^{g / /}$ mice expressing wtRas (A) 10x magnification (scale bar $=100 \mu \mathrm{m}$ ), (B) 20x magnification (scale bar $=50 \mu \mathrm{m}$ ) or oncKRas (C) 10x magnification (scale bar $=100 \mu \mathrm{m}$ ) and (D) 20x magnification (scale bar $=50 \mu \mathrm{m}$ ).

\subsubsection{Preliminary characterization of RMS after conditional expression of oncHRas in Ptch mutant mice}

In order to analyze the effects of oncogenic HRas (oncHRas) on RMS of Ptch mutant mice 60 Ptch $^{\text {del// }}$ HRas G12 $\mathrm{V}^{\mathrm{fl} / \mathrm{M}}$ Myf5CreERT $T^{\mathrm{tg} /-}$ mice were randomized in two groups. As also done with the other oncRas animals, one group was treated with $1 \mathrm{mg}$ tamoxifen on 5 consecutive days. The other group was left untreated. Figure 44 and Table 21 shows the data for approximately half of the animals. Although half of the mice is still living, the preliminary analysis reveals that compared to the controls oncHRas significantly increases the tumor incidence $(62.2 \% \mathrm{vs}$ $83.3 \%$, respectively) ( $P=0.0391$ for RMS by log-rank test), and significantly decreases the tumor-latency time (95 days vs 78 days, respectively) ( $P=0.0189$ for RMS by GehanBreslow-Wilcoxon test). It however does not influence the tumor multiplicity. 
Table 21: Influence of oncHRas on RMS development of Ptch ${ }^{\text {del/ }} H$ RasG $12 \mathrm{~V}^{\text {fl/- }}$ Myf5CreERT $T^{\text {tg/- }}$

\begin{tabular}{|c|c|c|c|c|c|c|}
\hline Genotype & $\begin{array}{c}\text { Status of } \\
\text { tamoxifen } \\
\text { induction }\end{array}$ & $\mathbf{n}_{\mathrm{A}}$ & $\begin{array}{l}\text { Mice } \\
\text { with } \\
\text { RMS }_{\mathrm{A}}\end{array}$ & $\begin{array}{c}\text { Mice with } \\
\text { multiple } \\
\text { RMS }_{\text {B }}\end{array}$ & $\begin{array}{c}\text { RMS number } \\
\text { per mouse } \\
(\text { mean } \pm \text { SEM })_{B}\end{array}$ & $\begin{array}{c}\text { Median } \\
\text { Latency } \\
\text { time of } \\
\text { RMS } \\
\text { (days) }\end{array}$ \\
\hline 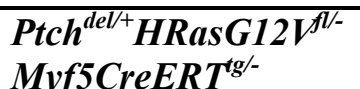 & uninduced & 30 & $\begin{array}{c}16 \\
(62.2 \%)\end{array}$ & $\begin{array}{c}6 \\
(46.2 \%)\end{array}$ & $1.615 \pm 0.241$ & 95 \\
\hline $\begin{array}{l}\text { Ptch }^{\text {del/ }} \text { HRasG } 12 V^{f / /-} \\
\text { Myf5CreERT }\end{array}$ & $\begin{array}{l}\text { tamoxifen- } \\
\text { induced }\end{array}$ & 31 & $\begin{array}{c}25 \\
(83.3 \%)\end{array}$ & $\begin{array}{c}7 \\
(46.6 \%)\end{array}$ & $1.583 \pm 0.119$ & 78 \\
\hline
\end{tabular}

$\mathrm{A}=$ all mice

$\mathrm{B}=$ mice that have been already subjected to autopsy

A

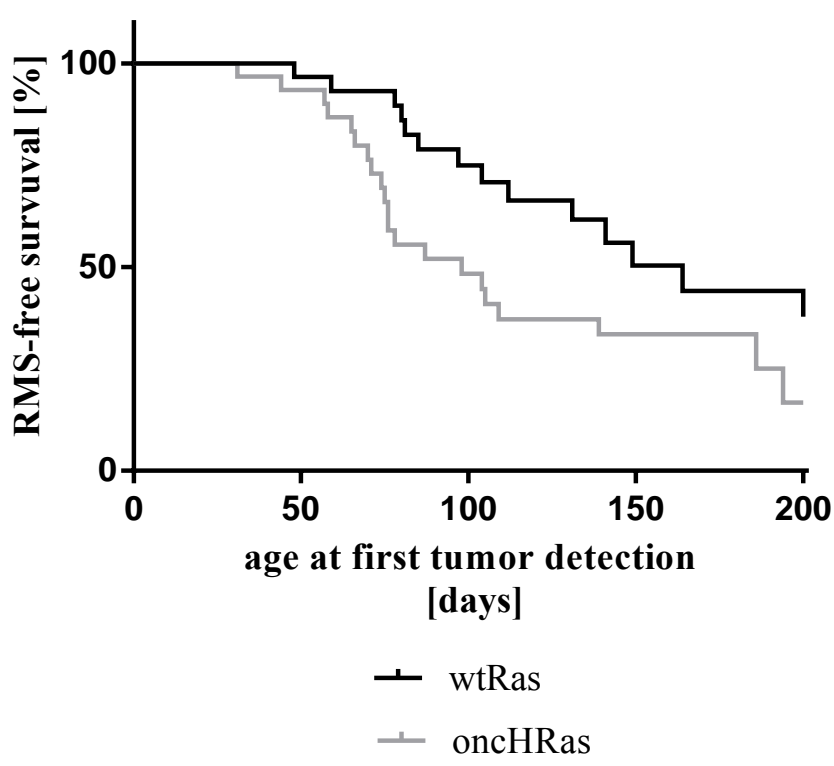

B

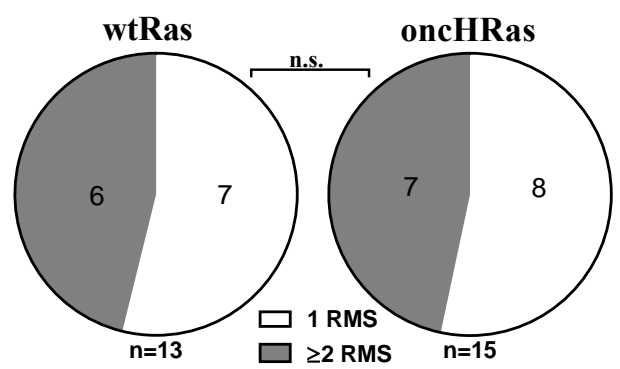

Figure 44: oncHRas significantly increases tumor incidence and shortens latency time. (A) Kaplan Mayer Curve with the RMS free survival of wtRas (black) and oncHRas (grey) Ptch ${ }^{d e l /+} H R a s G 12 V^{f l /-}$ Myf5CreERT $T^{\text {g/ }}$ mice. Every event represents the detection of the first RMS in a mouse. (B) Graph shows

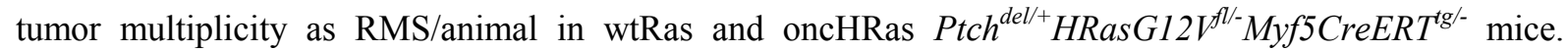
Statistical significance of the RMS-free survival was tested by log-rank test $(P=0.0391)$, of the latency time by Gehan-Breslow-Wilcoxon test $(P=0.0189)$ and of the multiplicity by Chi-squared test $(P=$ 0.4892). $* P<0.05$ 


\section{Discussion}

\subsection{Generation of RMS models with active Ras signaling in vivo and in vitro}

As already mentioned in the introduction activating mutations in RAS and active $\mathrm{HH}$ signaling are frequently found in human RMS (Paulson et al. 2011, Pressey et al. 2011, Zibat et al. 2010). Furthermore, these pathways are known to interact with each other. However, so far a connection of these two pathways in RMS has not been studied. The aim of this thesis was to investigate the interaction of Ras and Hh signaling in RMS. For this purpose, two experimental systems were established.

The first experimental system of this thesis was the establishment of stable cell lines for the in vitro analysis. These cell lines were used as tools to investigate the effects of oncogenic RAS signaling on HH signaling in RMS. Benefits of such cell lines are the possibility for simple manipulation, working with homogeneous material and the ability of various experiments within a short time.

It is known that active RAS signaling in cancer can have various outcomes depending on the tumor entity and can influence various functions of a cell. In this thesis we used ERMS and ARMS cell lines i.e. RUCH-2 and RMS-13, respectively, that are RAS wild type and that express major components of the HH signaling pathway. Despite the fact that so far just one case with an activating RAS mutation has been described in the alveolar subtype of RMS (Shern et al. 2014) we also checked the effects of RAS signaling on ARMS cells. With this experiment we hoped to get some insight into the mechanisms that prevent the prevalence of frequent RAS mutations in ARMS. These mechanisms could for example involve death of the cells due to apoptosis.

Since it is known that the three RAS proteins NRAS, KRAS and HRAS can differ in their posttranslational modifications and also can influence different cellular functions (Hancock 2003), cells expressing either oncogenic NRAS, KRAS or HRAS were generated and analyzed. In order to generate these cells, oncogenic RAS cDNA sequences, containing a glycine $(\mathrm{G})$ to valine $(\mathrm{V})$ substitution at residue $12(\mathrm{G} 12 \mathrm{~V})$ that constitutively activate RAS signaling, were cloned into a viral vector and stably transduced into in the above mentioned RMS cell lines. This should result in ectopical activation of RAS signaling in those cells. 
Indeed, both RUCH-2 and RMS-13 cells overexpressed RAS. RAS activity on the other hand was detected in RMS-13 cells by increased levels of pERK and in RUCH-2 cells by a RAS activation ELISA.

The second experimental system was the in vivo mouse models that allowed for the investigation of the effects of active Ras signaling on RMS. For this purpose the Ptch ${ }^{\text {del/ }}$ mouse model was employed. Based on histology these mice develop RMS resembling the human embryonal subtype. Furthermore, as human ERMS, these RMS show high Hh signaling activity, express Igf2 and show activity of PI3K/Akt signaling (Hahn et al. 2000, Kappler et al. 2003, Kappler et al. 2004). Thus, this mouse model is an ideal tool for studying murine RMS which share characteristics comparable to human ERMS. As mutations of all three subtypes of Ras were identified in human embryonal RMS (Stratton et al. 1989), distinct oncogenic Ras mouse models for each Ras subtype were generated, which namely were

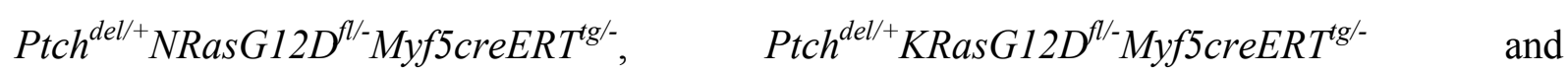
Ptch $^{\text {del/+ }}$ HRasG $12 \mathrm{~V}^{f l /-}$ Myf5creERT $T^{\text {tg/- }}$ mice.

When the mice were treated with tamoxifen, recombination of the lox sites occurred in oncNRas and oncKRas Ptch ${ }^{\text {del/+ }}$ oncRas $^{f l /-}$ Myf5CreERT $T^{\text {tg/- }}$ mice were comparable to those reported for other mouse models employing the respective oncRas mice (Haigis et al. 2008). Whereas, the recombination of the lox sites at the HRas locus could not be proven Ptch $^{\text {del/t }}$ HRasG12 $V^{f l /-}$ Myf5creERT ${ }^{t g /-}$ mice. For this purpose a new experimental setup remained to be established to investigate for correct recombination at the HRas locus. However, the recombination seems to be successful in all Ptch ${ }^{\mathrm{del} / \mathrm{+}}$ oncRas ${ }^{\mathrm{fl} / \mathrm{M}} \mathrm{Myf} 5 \mathrm{CreERT} \mathrm{T}^{\mathrm{tg} / \mathrm{-}}$ mice thus the tamoxifen-induction led to a RMS-specific increased Ras activity in induced compared to uninduced. Therefore these mouse lines were a useful tool for the investigation of the effects of active Ras signaling on RMS that show Hh-signaling activity.

\subsection{No obvious morphological changes of RMS by oncRas}

Active Ras signaling can have several effects on a tumor cell, including changes in the morphology. For example active HRas signaling was reported to change the morphology from flat-like to round-up-like of e.g. mouse NIH3T3 fibroblasts, normal rat kidney (NRK) cell and human MSU-1.1 cells (Yeh et al. 2008). The authors showed that active HRas signaling via activation of MEK/ERK mediates proteasome degradation of the signal transducer and activator of transcription (Stat3), which resulted in disruption of the microtubule in those 
cells. However, the activation of Ras signaling did not obviously change the morphology of

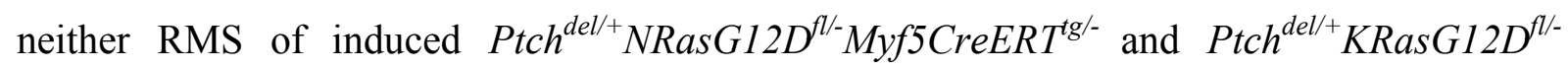
Myf5CreERT ${ }^{\text {tg/- }}$ mice nor of the cells and xenografts of the stably transduced RMS cell lines. This may indicate that oncRas signaling in RMS is not able to target the microtubule disruption by suppression of Stat3.

\subsection{Crosstalk of Ras and Hh signaling in RMS}

Several connections between the Ras and Hh signaling pathways have been shown in the past for example in lung, prostate and pancreas (reviewed by (Lauth 2011)). The modulations of active Ras signaling on Hh signaling can vary depending on the cancer and cell type. The outcome of such interaction can exhibit an activating as well as inhibiting effect on the activation state of these pathways (reviewed by (Aberger et al. 2012)). Since both pathways are active in RMS the potential crosstalk of RAS and HH signaling was investigated.

First, the effect of active Ras signaling on Hh signaling activity was analyzed. Indeed, oncRas altered the expression of Gli transcription factors both in human and in murine RMS. In the mouse, the effects of NRas and KRas were different. However, neither the NRas nor the KRas mutation significantly altered the expression level of Glil which is a potent read-out for active Hh signaling. (The analysis of the effect of the HRas mutation was not possible because Ptch $^{\text {del/ } /+}$ HRasG12D fl/- Myf5creERT $T^{\text {tg/- }}$ mice were still under observation).

In addition, active KRas signaling seems to slightly inhibit the expression of the Hh components Gli2 and Gli3 in Ptch $^{\text {del//+}}$ KRasG $12 D^{f l /-}$ Myf 5 creERT $T^{\text {tg/- }}$ mice, whereas in Ptch $^{\text {del// }}$ NRasG $12 D^{f l /-}$ Myf5creERT ${ }^{t g /-}$ mice the activation of NRas signaling resulted in significant upregulation of expression levels of Gli2 and Gli3. The latter observation is in line with previous investigations of our collaboration partners Marcel Kool and Simone Fulda on human RMS, who showed by microarray gene expression analysis that fusion-gene-negative RMS with active RAS signaling show an increased expression of the HH components, especially of GLI2. Furthermore, the array analysis showed that all fusion-gene-negative RMS samples that show a RAS signature are within the group showing active $\mathrm{HH}$ signaling. This was seen in three independent cohorts of RMS samples. These microarray data indicate that there is a positive correlation between both pathways in RMS, at least in human primary RMS samples. Since NRAS mutations are the most frequent ones in human RMS and since the 
microarray analysis did not distinguish between the individual RAS mutations in the samples, the mouse and human data may fit very nicely. Further analysis of the microarray data with discrimination between those samples with NRAS or KRAS mutations may allow for a better comparison of the mouse and human data.

Taken together the mouse data indicate that oncNRas and oncKRas can modulate the expression levels of components of the Hh pathway. The not significant results with respect to RAS/Ras-modulated GLI1/Gli1 expression levels - that is currently thought to be the best read-out for $\mathrm{HH} / \mathrm{Hh}$ signaling activity - hampers a clear statement about the influence of RAS mutations on $\mathrm{HH} / \mathrm{Hh}$ signaling activity in RMS. A clear statement probably requires a set of marker genes (instead of using only one i.e. GLII/Gli1 as a read-out for $\mathrm{HH} / \mathrm{Hh}$ signaling activity). Thus, in medulloblastoma $\mathrm{HH}$ signaling activity is demonstrated by a 5-genesignature that besides GLI1 includes PHK1, SHROOM2, PDLIM3, and OTX2 (Shou et al. 2015). Such a signature should also be established for RMS. In addition in situ hybridization assays for Hh signaling components should be performed. Together, this could finally help to draw a final conclusion about whether Hh signaling activity is modulated by oncRas or not.

Furthermore, the results from the in vivo experiments were different from those of the cell culture. Thus, the expression of all three RAS genes in cultured RUCH-2 and RMS-13 cells resulted in downregulation of the GLII transcription factors. This difference may be due to the fact that the cultured cells were not exposed to the tumor microenvironment that is well known to influence the tumor biology. Thus, the cells should be co-cultured with e.g. fibroblasts or bone-marrow-derived macrophages and the expression of the GLI transcription factors should be measured again.

Nevertheless, the fact that activation of RAS signaling decreased HH signaling activity in cultured RMS cell lines is similar to the RAS-mediated inhibition of HH signaling activity in cultured pancreatic carcinoma cells. In this cell line active KRAS signaling concomitantly suppressed the activity of the transcription factor GLI2 and GLI3 and thus inhibited HH signaling activity as demonstrated by downregulation of GLII expression. Furthermore oncKRAS simultaneously increased the expression and secretion of $\mathrm{SHH}$, which resulted in simultaneous activation of the surrounding tumor cells (Lauth et al. 2010). Gene expression analysis in RUCH-2 cells reveals the same effect on the expression levels of GLII (readout of 
HH signaling activity) and $S H H$. However, in contrast to pancreatic carcinoma cells RUCH-2 cells were not able to secrete SHH. Furthermore gene expression analysis in oncRAStransduced RUCH-2 cells in combination with specific inhibitors for canonical $\mathrm{HH}$, RAF/MEK/ERK and PI3K/AKT signaling provided evidence that GLII expression is regulated via MEK signaling, but not via canonical HH or PI3K signaling. This was due to the fact that blocking of canonical $\mathrm{HH}$ and PI3K signaling in the oncRAS cell lines did not reactivate GLII expression, whereas inhibition of MEK went along with the upregulation of the GLII expression back to the basal level of wtRAS control cells. In addition, since none of the RAS mutations by themselves altered the phosphorylation status of ERK the data suggest that ERK does not play a role in HH signaling activation.

The data also show that the MEK-inhibitor UO126 in NRAS- or KRAS-transduced RUCH-2 cells increased metabolic and proliferative capacity. This suggests that it is MEK activity that decreases the metabolic capacity and also inhibits the proliferative capacity of RAStransduced cells. These data therefore also suggest that MEK inhibitors are probably not a good treatment option in ERMS harboring oncogenic RAS mutations. Contrary effects of the MEK activation have been previously published by Seto and colleagues. These authors showed that KRAS-mediated MEK signaling positively, and not negatively, regulated GLII expression in gastric cancer cells (Seto et al. 2009). How active MEK signaling decreases HH signaling activity in RUCH-2 remains to be resolved. To further investigate the missing link between MEK and HH signaling in RUCH-2 cells, it first has to be analyzed if active MEK1 or MEK2 is responsible for this inhibition. As MEK1 has been shown to exhibit a stimulating effect on GLI1 transcription (see Seto and colleagues), it is possible that MEK2 is the kinase that inhibits the transcription of GLI1.

In the ARMS cell line RMS-13 and in contrast to RUCH-2, none of the two RAS effector pathways (i.e. MEK/ERK or PI3K/AKT signaling) seemed to be involved in inhibition of GLI1 expression. Thus, neither, HhA, PI103 nor UO126 were able to reverse the RASmediated downregulation of GLII expression. In addition, the fact that all three RAS oncogenes induced a strong phosphorylation of ERK that was reversed by UO126, again strongly argues against a role of ERK in GLII regulation. 
Since Lauth and colleagues showed that DYRK1B can be activated by RAS and can inhibit HH signaling in pancreas carcinoma cells, we next investigated the role of this kinase in RMS-13 cells. However, in contrast to Lauth et al. gene the expression analysis showed that oncogenic RAS signaling in the RMS-13 cells lead to the downregulation of the expression levels of DYRK1B. This downregulation was also detectable after knockdown DYRK1B in control cells and did not go along with upregulation of GLII, but rather with an even more pronounced downregulation of GLII. Together it can be said that RAS inhibits the expression of GLII by a jet unknown factor that is independent of RAF/MEK/ERK or PI3K/AKT signaling in RMS-13 cells.

Taken together there is a crosstalk between active Ras signaling and Hh signaling in RMS. However, this crosstalk seems to be differently regulated in ERMS and ARMS. In addition, the modulation of $\mathrm{HH} / \mathrm{Hh}$ signaling i.e. GLII/Glil expression by active Ras signaling is different between the ERMS cell line RUCH-2 and ERMS of mice. Thus, RAS-mediated inhibition of $\mathrm{HH}$ signaling activity was exclusively observed in cultured RUCH-2. Besides the possibility that the tumor microenvironment plays a role (see above) it is also possible that tumor cells in vivo may bypass the Ras-mediated inhibition of Hh signaling activity. This may involve additional genetically changes that may occur during tumor progression in the mouse.

In addition to the regulation of $\mathrm{HH}$ signaling by RAS, HH signaling activity seems to vice versa regulate RAS signaling, at least in the ERMS cell line RUCH-2. Thus, in RUCH-2 cells treatment with HhA decreased AKT/pAKT levels in both wtRAS and onRAS expressing cells. In addition, it increased phosphorylation of ERK in RUCH-2 cells that express oncRAS. Similar results have been demonstrated in RD cells that harbor an endogenous oncogenic NRAS mutation (Ridzewski et al. 2015).

The fact that HhA decreased AKT/pAKT levels in both wtRAS and onRAS expressing cells, but only it increased phosphorylation of ERK in onRAS-expressing cells RUCH-2 cells is hard to explain. However, a cross-inhibition between RAF/MEK/ERK and PI3K/AKT signaling pathways is well known. As reviewed by Mendoza et al such a crosstalk occurs mostly when one of the pathways is chemically blocked by a specific inhibitor. This results in the activation of the other pathway (Mendoza et al. 2011). A cross-inhibition can be seen between AKT and RAF. AKT can negatively regulate ERK activation by phosphorylation of 
the inhibitory sites in the Raf N-terminus (Cheung et al. 2008, Dhillon et al. 2002, Guan et al. 2000, Zimmermann and Moelling 1999). Furthermore, it has been shown that active $\mathrm{PI} 3 \mathrm{~K} / \mathrm{AKT}$ signaling can inhibit the activation of oncRAS mediated MEK/ERK signaling, which prevents cell cycle arrest or senescence caused by too high levels of phosphorylated ERK (Cheung et al. 2008).

Our data reveals that the inhibition of $\mathrm{HH}$ signaling with $\mathrm{HhA}$ in parallel inhibits the phosphorylation of AKT and increases phosphorylation of ERK, especially in embryonal RMS cell lines with oncRAS signaling. Based on these data one can hypothesize that HhA inhibits canonical Hedgehog signaling and somehow the phosphorylation of AKT. This in turn blocks the ability of AKT to phosphorylate the inhibitory sites in the RAF N-terminus, which results in strong induction of ERK phosphorylation especially in oncRAS RUCH-2 cells.

A different effect can be seen in RMS-13 cells. Thus, AKT phosphorylation went up after inhibition of MEK by UO126 in the oncRAS as well as in the wtRAS ARMS cells. MEK inhibitors can increase AKT activation by inducing epidermal growth factor (EGF)-mediated pathway activation (Hoeflich et al. 2009, Yu et al. 2002). It is expected that normally the phosphorylation of ERK inhibits GAB, which is responsible for the recruitment of PI3K to the EGF receptor (EGFR) and thus increases PI3K signaling (Lemmon and Schlessinger 2010, Wohrle et al. 2009).

\subsection{Active Ras signaling changes the growth behavior in RMS}

It has been shown that active Ras signaling can change the growth behavior of tumors in other cancers types like colon, lung and prostate cancers (Erlich et al. 2006, Haigis et al. 2008, Hofmann et al. 2012, Johnson et al. 2001). Despite the fact that the RAS genes are highly homologue they can act differently.

Indeed, expression of oncogenic NRas, KRas and HRas genes results in gene-specific differences with respect to RMS growth. While oncNRas had no effects neither on incidence nor latency time of RMS in Ptch mutant mice, oncKRas signaling strongly increased the RMS incidence and decreased the latency time. Furthermore preliminary data of RMS development after conditional expression of oncHRas in Ptch mutant mice provide evidence that oncHRas, 
as oncKRas, strongly increases the tumor incidence and decreases the latency time. This shows that oncogenic KRas and HRas mutations have a similar impact on RMS incidence and latency time, whereas the expression of the oncNRas mutation apparently does not influence these parameters. This suggests that oncKRas and oncHRas may not influence the growth of already initiated RMS. Due to the fact that RMS in Ptch mutant mice are initiated during embryogenesis during a small window that closes at embryonic day 13.5 (Nitzki et al. 2011). Nevertheless, it is also possible that oncHRas and oncKRas may initiate new tumors after birth (i.e. in 4 week old mice, when the mutation is induced) in the Ptch deficient organism.

Very unfortunately, the size of the tumors was not measured. Therefore it was not possible to judge whether the tumors harboring the respective oncRas mutations grew faster or slower after their initiation. For example the oncNRas mutation in RMS of Ptch-mutant mice did not influence incidence and latency time of the tumor. It however, and in contrast to oncKRas, lowered the number of Ki67 positive RMS cells. Thus, it is possible that the NRas mutation may have reduced the growth of RMS and may have resulted in smaller tumor volumes.

Regarding the lower numbers of Ki67 positive RMS cells in the $\operatorname{Ptch}^{\mathrm{del} / \mathrm{A}} \mathrm{NR}$ as G12D $D^{f l-}$ $M y f 5 \mathrm{creERT} T^{\mathrm{tg} / \mathrm{C}}$ model and the unchanged number of Ki67 positive cells in Ptch $^{\text {del//+}}$ KRasG $12 D^{f l /-}$ Myf5creERT ${ }^{t g /-}$ mice it remains to be said that this is different in colon cancer mouse models where oncKRas induces hyperproliferation and oncNRas does not change the proliferative capacity (Haigis et al. 2008).

With respect to the increased RMS incidence and shortening of the latency time of RMS in Ptch $^{\text {del/t+}}$ KRasG12D $D^{f l /}$ Myf5creERT $T^{\text {tg/- }}$ mice it was reported that active KRAS and HH signaling also increase the number of pancreatic intraepithelial tumors and increase the lethality of $P d x$ Cre;CLEG2;KrasG12D mice (Pasca di Magliano et al. 2006). However, how oncKRas modulates the tumorigenesis in Ptch mutant mice remains to be identified. As published by our group and others it is expected that the formation of human and murine rhabdomyoblastic tumors, which harbor a heterozygous mutation in Ptch, necessitates the epigenetic silencing of the remaining wt Ptch allele (Ecke et al. 2009, Tostar et al. 2006, Uhmann et al. 2005). The formation of RMS by the epigenetic silencing of the wt Ptch allele depends in the DNA methylation thus it can be suppressed by the treatment of the DNA methyltransferase (Dnmt) inhibitor 5-aza-20deoxycytidin (Ecke et al. 2009). Furthermore, we could recently demonstrated that the RMS formation in Ptch heterozygous mice requires the overexpression 
of the mutant Ptch allele which is associated with the promoter hypomethylation of the mutant Ptch allele (Nitzki et al. 2015). Interestingly, oncogenic RAS signaling has often been shown to be able to modulate DNA methylation by the upregulation of DNMT expression and promotion of cellular DNMTs activity. This induces a RAS-mediated hypomethylation (reviewed by (Mei et al. 2006). Thus, it is possible that oncKRas in Ptch mutant mice either promotes the overexpression of Dnmt which would result in the hypomethylation of the mutant Ptch allele or the process to silence the wt Ptch allele and thus increases the tumor incidence and decreases the latency time without affecting the proliferation.

Additionally to the tumor incidence and latency time the tumor multiplicity was investigated. Interestingly, activated NRas decreased the tumor multiplicity in the $\operatorname{Ptch}^{\mathrm{del} / \mathrm{+}}$ oncNRas $^{\mathrm{fl} / \mathrm{-}}$ Myf5creERT $T^{\text {tg/- }}$ mouse model, whereas the KRas and HRas mutations did not. The decrease of the tumor number per Ptch $^{d e l /+}$ oncNRas ${ }^{f l /-} M y f 5 c_{r e E R T} T^{t /-}$ mouse fits nicely to the fact that oncNRas suppressed the proliferative capacity of RMS.

A hypothesis for the decreased proliferative capacity in oncNRas expressing RMS might be oncogene-induced senescence (OIS). Thus, it was reported, that oncogenic Ras signaling can lead to OIS by accumulation of p53 and p16 (Serrano et al. 1997). It was shown that the inhibition of either p53 or p16 reversed the Ras-mediated senescence in human and primary human or rodent cells. Furthermore Courtois-Cox et al. reported that there was an upregulation of several senescence markers in benign dermal neurofibromas after the suppression of NF1. In this model, loss of NF1 resulted in activation of RAS that was responsible for senescence (Courtois-Cox et al. 2006). However, if OIS is responsible for the decreased proliferative capacity in the oncNRas expressing Ptch mutant RMS remains to be validated. Thus, the tissue of those animals should be analyzed for different senescence markers i.e. by performing a senescence-associated $\beta$-galactosidase (SA- $\beta$-Gal) staining and by checking p53 and p16.

Taken together these results indicate that, although the RAS genes code for highly homologous RAS proteins, N-, K- and HRAS mutations can induce different phenotypes of murine and human RMS, especially of the embryonal subtype.

In order to identify, which downstream targets of oncogenic NRas, KRas and HRas in Ptch mutant mice are responsible for the differences in RMS incidence, latency time or multiplicity, one could perform an array assay. 
At the moment the evidence of dual inhibition of RAS and HH targets is frequency discussed (Brechbiel et al. 2014). Whether it makes sense to inhibit both RAS signaling and HH signaling in RMS remains to be established. However so far it can be concluded that it is of importance to discriminate between the three RAS subtypes. Thus, in our mouse model, oncNRas seems to improve the prognosis (since it decreases the proliferation and tumor multiplicity), whereas oncKRas worsen the prognosis. This is in line with an unpublished Kaplan Mayer Curve of our collaborative project together with Marcel Kool and Simone Fulda that reveals that ERMS patients (many of them having NRAS mutations) with active RAS and HH signaling seem to have a better prognosis compared to those without.

In contrast to the embryonal subtype, oncogenic RAS signaling in the alveolar RMS subtype results in the enhancement of the tumor growth in nude mice and of proliferation and metabolic activity in vitro. The injected tumor cells with constitutively active forms of NRAS and KRAS showed an increased tumor growth rate in the xenograft model compared to the control cells.

When the ARMS cell line RMS-13 was incubated with inhibitors, the results revealed that HhA and PI103, but not UO126, can inhibit proliferation and metabolic activity of control cells, which indicated that proliferation of RMS-13 cells may be regulated by $\mathrm{HH}$ and PI3K/mTOR signaling. HhA and PI103 also repressed proliferation and metabolic activity in RMS-13 cells that express oncogenic NRAS, KRAS and HRAS. This was different for UO126. This MEK inhibitor repressed proliferation only in oncHRAS-transduced cells and metabolic activity in oncNRAS and oncHRAS transduced cells.

These results are hard to explain. However, it is possible that it is again MEK that decreases proliferation and metabolic activity in the settings in which ARMS cells express oncogenic RAS. In wtRAS RMS-13 cells, however, inhibition of MEK is not useful to stop proliferation. Since most ARMS do not harbor oncRAS mutations MEK inhibitors thus also do not appear to be a good treatment option for this RMS subtype. Furthermore, UO126 decreased pERK levels in wtRAS RMS-13 cells. This indicates that the drug must have inhibited MEK. It also indicates that the proliferation of wtRAS RMS-13 cells is independent of the MEK/ERK axis 


\subsection{Active Ras signaling slightly modulates the muscle differentiation behavior in RMS}

Several studies suggest that oncogenic RAS result in the dysregulation of the differentiation behavior in tumors. Thus, in a myogenic cell line constitutive activation of Ras signaling resulted in downregulation of the myogenic differentiation markers MyoD and Myogenin (Konieczny et al. 1989). Furthermore other studies suggest that the change in muscle differentiation is regulated via RAS-mediated PI3K signaling (Lee et al. 2010).

When the expression of the early myogenesis marker $M Y O D$ and the late muscle differentiation marker DESMIN were measured in wtRAS and oncRAS expressing cell lines, no significant differences were detected. Thus RUCH-2 merely express any of these markers, and the expression was slightly, but not significantly enhanced by oncRAS in RMS-13 cells.

Next, the expression levels of the myogenesis proliferation and determination markers $M y o D$ and Myf5 and the differentiation marker Myogenin were measured in RMS from uninduced and tamoxifen-induced Ptch $^{\text {del/ }}$ oncRas ${ }^{f l /-}$ Myf5creERT ${ }^{t g /-}$ mice. Here, the effects on the expression of these muscle differentiation markers were dependent on the respective Ras mutation. Our preliminary expression analysis provides evidence that oncNRas signaling rather suppresses the expression of the myogenesis proliferation and muscle differentiation markers in Ptch mutant RMS. This is comparable to the effects of oncogenic Ras signaling in murine muscle cells. Although the decrease in the expression of muscle differentiation markers is rather indicative for less differentiated cells, the RMS histologically did not obviously differ from control RMS (Lassar et al. 1989, Olson et al. 1987). In contrast, oncKRas rather seems to increase the expression levels of the early markers $M y o D$ and $M y f 5$ and to decrease the expression of the late differentiation marker Myogenin in Ptch mutant RMS, which may indicate that the RMS cells still maintain their muscle fate but lose the possibility for terminal differentiation. However, the differences in myogenesis proliferation and determination markers were not significant thus indicating that oncRas signaling probably does not modulate the muscle differentiation behavior in RMS. In order to prove whether oncRas influences the muscle differentiation behavior in RMS gene expression analysis of more mice and IHC stainings with the above mentioned muscle differentiation markers should be performed. 


\section{References}

Aberger F, Kern D, Greil R, Hartmann TN (2012). Canonical and noncanonical Hedgehog/GLI signaling in hematological malignancies. Vitam Horm 88: 25-54.

Aksamitiene E, Kiyatkin A, Kholodenko BN (2012). Cross-talk between mitogenic Ras/MAPK and survival PI3K/Akt pathways: a fine balance. Biochem Soc Trans 40: 139-146.

Aza-Blanc P, Ramirez-Weber FA, Laget MP, Schwartz C, Kornberg TB (1997). Proteolysis that is inhibited by hedgehog targets Cubitus interruptus protein to the nucleus and converts it to a repressor. Cell 89: 1043-1053.

Beachy PA, Karhadkar SS, Berman DM (2004). Tissue repair and stem cell renewal in carcinogenesis. Nature 432: 324-331.

Biressi S, Bjornson CR, Carlig PM, Nishijo K, Keller C, Rando TA (2013). Myf5 expression during fetal myogenesis defines the developmental progenitors of adult satellite cells. Developmental biology 379: 195-207.

Bitgood MJ, Shen L, McMahon AP (1996). Sertoli cell signaling by Desert hedgehog regulates the male germline. Current biology : $C B$ 6: 298-304.

Boguski MS, McCormick F (1993). Proteins regulating Ras and its relatives. Nature 366: 643-654.

Bollag G, McCormick F (1991). Regulators and effectors of ras proteins. Annual review of cell biology 7: 601-632.

Bourne HR, Sanders DA, McCormick F (1990). The GTPase superfamily: a conserved switch for diverse cell functions. Nature 348: 125-132.

Bourne HR, Sanders DA, McCormick F (1991). The GTPase superfamily: conserved structure and molecular mechanism. Nature 349: 117-127.

Bozulic L, Hemmings BA (2009). PIKKing on PKB: regulation of PKB activity by phosphorylation. Current opinion in cell biology 21: 256-261.

Brechbiel J, Miller-Moslin K, Adjei AA (2014). Crosstalk between hedgehog and other signaling pathways as a basis for combination therapies in cancer. Cancer treatment reviews 40: 750-759.

Breneman JC, Lyden E, Pappo AS, Link MP, Anderson JR, Parham DM et al (2003). Prognostic factors and clinical outcomes in children and adolescents with metastatic rhabdomyosarcoma--a report from the Intergroup Rhabdomyosarcoma Study IV. Journal of clinical oncology : official journal of the American Society of Clinical Oncology 21: 78-84.

Bridge JA, Liu J, Weibolt V, Baker KS, Perry D, Kruger R et al (2000). Novel genomic imbalances in embryonal rhabdomyosarcoma revealed by comparative genomic 
hybridization and fluorescence in situ hybridization: an intergroup rhabdomyosarcoma study. Genes Chromosomes Cancer 27: 337-344.

Bridge JA, Liu J, Qualman SJ, Suijkerbuijk R, Wenger G, Zhang J et al (2002). Genomic gains and losses are similar in genetic and histologic subsets of rhabdomyosarcoma, whereas amplification predominates in embryonal with anaplasia and alveolar subtypes. Genes Chromosomes Cancer 33: 310-321.

Briscoe J, Therond PP (2013). The mechanisms of Hedgehog signalling and its roles in development and disease. Nature reviews Molecular cell biology 14: 416-429.

Chen JK, Taipale J, Young KE, Maiti T, Beachy PA (2002). Small molecule modulation of Smoothened activity. Proceedings of the National Academy of Sciences of the United States of America 99: 14071-14076.

Chen JY, Lin JR, Cimprich KA, Meyer T (2012). A two-dimensional ERK-AKT signaling code for an NGF-triggered cell-fate decision. Molecular cell 45: 196-209.

Chen X, Mitsutake N, LaPerle K, Akeno N, Zanzonico P, Longo VA et al (2009). Endogenous expression of Hras $(\mathrm{G} 12 \mathrm{~V})$ induces developmental defects and neoplasms with copy number imbalances of the oncogene. Proceedings of the National Academy of Sciences of the United States of America 106: 7979-7984.

Chen X, Stewart E, Shelat AA, Qu C, Bahrami A, Hatley M et al (2013). Targeting oxidative stress in embryonal rhabdomyosarcoma. Cancer cell 24: 710-724.

Cheung M, Sharma A, Madhunapantula SV, Robertson GP (2008). Akt3 and mutant V600E B-Raf cooperate to promote early melanoma development. Cancer research 68: 34293439 .

Cohen MM, Jr. (2010). Hedgehog signaling update. Am J Med Genet A 152A: 1875-1914.

Courtois-Cox S, Genther Williams SM, Reczek EE, Johnson BW, McGillicuddy LT, Johannessen CM et al (2006). A negative feedback signaling network underlies oncogene-induced senescence. Cancer cell 10: 459-472.

Courtois-Cox S, Jones SL, Cichowski K (2008). Many roads lead to oncogene-induced senescence. Oncogene 27: 2801-2809.

Cowley S, Paterson H, Kemp P, Marshall CJ (1994). Activation of MAP kinase kinase is necessary and sufficient for PC12 differentiation and for transformation of NIH 3T3 cells. Cell 77: 841-852.

Dagher R, Helman L (1999). Rhabdomyosarcoma: an overview. Oncologist 4: 34-44.

De Giovanni C, Landuzzi L, Nicoletti G, Lollini PL, Nanni P (2009). Molecular and cellular biology of rhabdomyosarcoma. Future Oncol 5: 1449-1475.

Dent P, Haser W, Haystead TA, Vincent LA, Roberts TM, Sturgill TW (1992). Activation of mitogen-activated protein kinase kinase by v-Raf in NIH 3T3 cells and in vitro [see comments]. Science 257: 1404-1407. 
Der CJ, Krontiris TG, Cooper GM (1982). Transforming genes of human bladder and lung carcinoma cell lines are homologous to the ras genes of Harvey and Kirsten sarcoma viruses. Proceedings of the National Academy of Sciences of the United States of America 79: 3637-3640.

Dhillon AS, Meikle S, Yazici Z, Eulitz M, Kolch W (2002). Regulation of Raf-1 activation and signalling by dephosphorylation. The EMBO journal 21: 64-71.

Dijkgraaf GJ, Alicke B, Weinmann L, Januario T, West K, Modrusan Z et al (2011). Small molecule inhibition of GDC-0449 refractory smoothened mutants and downstream mechanisms of drug resistance. Cancer research 71: 435-444.

Downward J (1998). Ras signalling and apoptosis. Current opinion in genetics \& development 8: 49-54.

Downward J (2003). Targeting RAS signalling pathways in cancer therapy. Nature reviews Cancer 3: 11-22.

Downward J (2004). PI 3-kinase, Akt and cell survival. Seminars in cell \& developmental biology 15: 177-182.

Doyle B, Morton JP, Delaney DW, Ridgway RA, Wilkins JA, Sansom OJ (2010). p53 mutation and loss have different effects on tumourigenesis in a novel mouse model of pleomorphic rhabdomyosarcoma. J Pathol 222: 129-137.

Echelard Y, Epstein DJ, St-Jacques B, Shen L, Mohler J, McMahon JA et al (1993). Sonic hedgehog, a member of a family of putative signaling molecules, is implicated in the regulation of CNS polarity. Cell 75: 1417-1430.

Ecke I, Petry F, Rosenberger A, Tauber S, Monkemeyer S, Hess I et al (2009). Antitumor effects of a combined 5-aza-2'deoxycytidine and valproic acid treatment on rhabdomyosarcoma and medulloblastoma in Ptch mutant mice. Cancer research 69: 887-895.

Erlich S, Tal-Or P, Liebling R, Blum R, Karunagaran D, Kloog Y et al (2006). Ras inhibition results in growth arrest and death of androgen-dependent and androgen-independent prostate cancer cells. Biochemical pharmacology 72: 427-436.

Fan QW, Knight ZA, Goldenberg DD, Yu W, Mostov KE, Stokoe D et al (2006). A dual PI3 kinase/mTOR inhibitor reveals emergent efficacy in glioma. Cancer cell 9: 341-349.

Favata MF, Horiuchi KY, Manos EJ, Daulerio AJ, Stradley DA, Feeser WS et al (1998). Identification of a novel inhibitor of mitogen-activated protein kinase kinase. The Journal of biological chemistry 273: 18623-18632.

Feig LA, Buchsbaum RJ (2002). Cell signaling: life or death decisions of ras proteins. Curr Biol 12: R259-261.

Franke TF (2008). PI3K/Akt: getting it right matters. Oncogene 27: 6473-6488. 
Graham ES, Ball N, Scotter EL, Narayan P, Dragunow M, Glass M (2006). Induction of Krox-24 by endogenous cannabinoid type 1 receptors in Neuro2A cells is mediated by the MEK-ERK MAPK pathway and is suppressed by the phosphatidylinositol 3kinase pathway. The Journal of biological chemistry 281: 29085-29095.

Guan KL, Figueroa C, Brtva TR, Zhu T, Taylor J, Barber TD et al (2000). Negative regulation of the serine/threonine kinase B-Raf by Akt. The Journal of biological chemistry 275: 27354-27359.

Guenther MK, Graab U, Fulda S (2013). Synthetic lethal interaction between $\mathrm{PI3K} / \mathrm{Akt} / \mathrm{mTOR}$ and Ras/MEK/ERK pathway inhibition in rhabdomyosarcoma. Cancer letters 337: 200-209.

Gupta S, Takebe N, Lorusso P (2010). Targeting the Hedgehog pathway in cancer. Therapeutic advances in medical oncology 2: 237-250.

Hahn H, Wicking C, Zaphiropoulous PG, Gailani MR, Shanley S, Chidambaram A et al (1996). Mutations of the human homolog of Drosophila patched in the nevoid basal cell carcinoma syndrome. Cell 85: 841-851.

Hahn H, Wojnowski L, Zimmer AM, Hall J, Miller G, Zimmer A (1998). Rhabdomyosarcomas and radiation hypersensitivity in a mouse model of Gorlin syndrome. Nature Med 4: 619-622.

Hahn H, Wojnowski L, Specht K, Kappler R, Calzada-Wack J, Potter D et al (2000). Patched target Igf2 is indispensable for the formation of medulloblastoma and rhabdomyosarcoma. The Journal of biological chemistry 275: 28341-28344.

Hahn H, Nitzki F, Schorban T, Hemmerlein B, Threadgill D, Rosemann M (2004). Genetic mapping of a Ptch1-associated rhabdomyosarcoma susceptibility locus on mouse chromosome 2. Genomics 84: 853-858.

Haigis KM, Kendall KR, Wang Y, Cheung A, Haigis MC, Glickman JN et al (2008). Differential effects of oncogenic K-Ras and N-Ras on proliferation, differentiation and tumor progression in the colon. Nature genetics 40: 600-608.

Hancock JF (2003). Ras proteins: different signals from different locations. Nature reviews Molecular cell biology 4: 373-384.

Hoeflich KP, O'Brien C, Boyd Z, Cavet G, Guerrero S, Jung K et al (2009). In vivo antitumor activity of MEK and phosphatidylinositol 3-kinase inhibitors in basal-like breast cancer models. Clinical cancer research : an official journal of the American Association for Cancer Research 15: 4649-4664.

Hofmann I, Weiss A, Elain G, Schwaederle M, Sterker D, Romanet V et al (2012). K-RAS mutant pancreatic tumors show higher sensitivity to MEK than to PI3K inhibition in vivo. PloS one 7: e44146.

Hooper JE, Scott MP (2005). Communicating with Hedgehogs. Nature reviews Molecular cell biology 6: 306-317. 
Ji Z, Mei FC, Xie J, Cheng X (2007). Oncogenic KRAS activates hedgehog signaling pathway in pancreatic cancer cells. The Journal of biological chemistry 282: 1404814055.

Johnson L, Mercer K, Greenbaum D, Bronson RT, Crowley D, Tuveson DA et al (2001). Somatic activation of the K-ras oncogene causes early onset lung cancer in mice. Nature 410: 1111-1116.

Kappler R, Calzada-Wack J, Schnitzbauer U, Koleva M, Herwig A, Piontek G et al (2003). Molecular characterization of Patched-associated rhabdomyosarcoma. J Pathol 200: 348-356.

Kappler R, Bauer R, Calzada-Wack J, Rosemann M, Hemmerlein B, Hahn H (2004). Profiling the molecular difference between Patched- and p53-dependent rhabdomyosarcoma. Oncogene 23: 8785-8795.

Kasper M, Schnidar H, Neill GW, Hanneder M, Klingler S, Blaas L et al (2006). Selective modulation of Hedgehog/GLI target gene expression by epidermal growth factor signaling in human keratinocytes. Molecular and cellular biology 26: 6283-6298.

Knight ZA, Gonzalez B, Feldman ME, Zunder ER, Goldenberg DD, Williams O et al (2006). A pharmacological map of the PI3-K family defines a role for p110alpha in insulin signaling. Cell 125: 733-747.

Konieczny SF, Drobes BL, Menke SL, Taparowsky EJ (1989). Inhibition of myogenic differentiation by the H-ras oncogene is associated with the down regulation of the MyoD1 gene. Oncogene 4: 473-481.

Kyriakis JM, App H, Zhang XF, Banerjee P, Brautigan DL, Rapp UR et al (1992). Raf-1 activates MAP kinase-kinase. Nature 358: 417-421.

Lakso M, Pichel JG, Gorman JR, Sauer B, Okamoto Y, Lee E et al (1996). Efficient in vivo manipulation of mouse genomic sequences at the zygote stage. Proceedings of the National Academy of Sciences of the United States of America 93: 5860-5865.

Langenau DM, Keefe MD, Storer NY, Guyon JR, Kutok JL, Le X et al (2007). Effects of RAS on the genesis of embryonal rhabdomyosarcoma. Genes \& development 21: 1382-1395.

Lassar AB, Thayer MJ, Overell RW, Weintraub H (1989). Transformation by activated ras or fos prevents myogenesis by inhibiting expression of MyoD1. Cell 58: 659-667.

Lauth M, Bergstrom A, Shimokawa T, Tostar U, Jin Q, Fendrich V et al (2010). DYRK1Bdependent autocrine-to-paracrine shift of Hedgehog signaling by mutant RAS. Nature structural \& molecular biology 17: 718-725.

Lauth M (2011). RAS and Hedgehog--partners in crime. Front Biosci (Landmark Ed) 16: 2259-2270. 
Lee J, Choi KJ, Lim MJ, Hong F, Choi TG, Tak E et al (2010). Proto-oncogenic H-Ras, KRas, and N-Ras are involved in muscle differentiation via phosphatidylinositol 3kinase. Cell research 20: 919-934.

Lemmon MA, Schlessinger J (2010). Cell signaling by receptor tyrosine kinases. Cell 141: 1117-1134.

Linder B, (2015) "Interaction of the Hedgehog and vitamin D receptor signaling pathways in Patched associated cancers" Dissertation

Liu H, Gu D, Xie J (2011). Clinical implications of hedgehog signaling pathway inhibitors. Chinese journal of cancer 30: 13-26.

Mao J, Ligon KL, Rakhlin EY, Thayer SP, Bronson RT, Rowitch D et al (2006). A novel somatic mouse model to survey tumorigenic potential applied to the Hedgehog pathway. Cancer research 66: 10171-10178.

Marais R, Light Y, Paterson HF, Marshall CJ (1995). Ras recruits Raf-1 to the plasma membrane for activation by tyrosine phosphorylation. The EMBO journal 14: 31363145 .

Marigo V, Scott MP, Johnson RL, Goodrich LV, Tabin CJ (1996). Conservation in hedgehog signaling: induction of a chicken patched homolog by Sonic hedgehog in the developing limb. Development 122: 1225-1233.

Marigo V, Tabin CJ (1996). Regulation of patched by sonic hedgehog in the developing neural tube. Proceedings of the National Academy of Sciences of the United States of America 93: 9346-9351.

Martelli AM, Evangelisti C, Chiarini F, Grimaldi C, Cappellini A, Ognibene A et al (2010). The emerging role of the phosphatidylinositol 3-kinase/Akt/mammalian target of rapamycin signaling network in normal myelopoiesis and leukemogenesis. Biochimica et biophysica acta 1803: 991-1002.

Martinelli S, McDowell HP, Vigne SD, Kokai G, Uccini S, Tartaglia M et al (2009). RAS signaling dysregulation in human embryonal Rhabdomyosarcoma. Genes Chromosomes Cancer 48: 975-982.

Mebratu Y, Tesfaigzi Y (2009). How ERK1/2 activation controls cell proliferation and cell death: Is subcellular localization the answer? Cell Cycle 8: 1168-1175.

Mei FC, Young TW, Liu J, Cheng X (2006). RAS-mediated epigenetic inactivation of OPCML in oncogenic transformation of human ovarian surface epithelial cells. FASEB journal : official publication of the Federation of American Societies for Experimental Biology 20: 497-499.

Mendoza MC, Er EE, Blenis J (2011). The Ras-ERK and PI3K-mTOR pathways: cross-talk and compensation. Trends in biochemical sciences 36: 320-328. 
Niewiadomski P, Kong JH, Ahrends R, Ma Y, Humke EW, Khan S et al (2014). Gli protein activity is controlled by multisite phosphorylation in vertebrate Hedgehog signaling. Cell reports 6: 168-181.

Nitzki F, (2008) "Patched-assoziierte Tumoren: Modifikatorgene und Pathogenese" Dissertation

Nitzki F, Zibat A, Frommhold A, Schneider A, Schulz-Schaeffer W, Braun T et al (2011). Uncommitted precursor cells might contribute to increased incidence of embryonal rhabdomyosarcoma in heterozygous Patched1-mutant mice. Oncogene 30: 4428-4436.

Nitzki F, Tolosa EJ, Cuvelier N, Frommhold A, Salinas-Riester G, Johnsen SA et al (2015). Overexpression of mutant Ptch in rhabdomyosarcomas is associated with promoter hypomethylation and increased Gli1 and H3K4me3 occupancy. Oncotarget 6: 9113 9124.

Nusslein-Volhard C, Wieschaus E (1980). Mutations affecting segment number and polarity in Drosophila. Nature 287: 795-801.

Olson EN, Spizz G, Tainsky MA (1987). The oncogenic forms of N-ras or H-ras prevent skeletal myoblast differentiation. Molecular and cellular biology 7: 2104-2111.

Parada LF, Tabin CJ, Shih C, Weinberg RA (1982). Human EJ bladder carcinoma oncogene is homologue of Harvey sarcoma virus ras gene. Nature 297: 474-478.

Pasca di Magliano M, Sekine S, Ermilov A, Ferris J, Dlugosz AA, Hebrok M (2006). Hedgehog/Ras interactions regulate early stages of pancreatic cancer. Genes \& development 20: 3161-3173.

Paulson V, Chandler G, Rakheja D, Galindo RL, Wilson K, Amatruda JF et al (2011). Highresolution array $\mathrm{CGH}$ identifies common mechanisms that drive embryonal rhabdomyosarcoma pathogenesis. Genes Chromosomes Cancer 50: 397-408.

Pressey JG, Anderson JR, Crossman DK, Lynch JC, Barr FG (2011). Hedgehog pathway activity in pediatric embryonal rhabdomyosarcoma and undifferentiated sarcoma: A report from the Children's Oncology Group. Pediatric blood \& cancer.

Ridzewski R, Rettberg D, Dittmann K, Cuvelier N, Fulda S, Hahn H (2015). Hedgehog Inhibitors in Rhabdomyosarcoma: A Comparison of Four Compounds and Responsiveness of Four Cell Lines. Frontiers in oncology 5: 130.

Riobo NA, Haines GM, Emerson CP, Jr. (2006a). Protein kinase C-delta and mitogenactivated protein/extracellular signal-regulated kinase-1 control GLI activation in hedgehog signaling. Cancer research 66: 839-845.

Riobo NA, Lu K, Ai X, Haines GM, Emerson CP, Jr. (2006b). Phosphoinositide 3-kinase and Akt are essential for Sonic Hedgehog signaling. Proceedings of the National Academy of Sciences of the United States of America 103: 4505-4510. 
Roberg-Larsen H, Strand MF, Krauss S, Wilson SR (2014). Metabolites in vertebrate Hedgehog signaling. Biochemical and biophysical research communications 446: 669-674.

Robinson MJ, Cheng M, Khokhlatchev A, Ebert D, Ahn N, Guan KL et al (1996). Contributions of the mitogen-activated protein (MAP) kinase backbone and phosphorylation loop to MEK specificity. The Journal of biological chemistry 271: 29734-29739.

Rohatgi R, Milenkovic L, Scott MP (2007). Patched1 regulates hedgehog signaling at the primary cilium. Science 317: 372-376.

Roop DR, Lowy DR, Tambourin PE, Strickland J, Harper JR, Balaschak M et al (1986). An activated Harvey ras oncogene produces benign tumours on mouse epidermal tissue. Nature 323: 822-824.

Rubin BP, Nishijo K, Chen HI, Yi X, Schuetze DP, Pal R et al (2011). Evidence for an Unanticipated Relationship between Undifferentiated Pleomorphic Sarcoma and Embryonal Rhabdomyosarcoma. Cancer cell 19: 177-191.

Rudalska R, Dauch D, Longerich T, McJunkin K, Wuestefeld T, Kang TW et al (2014). In vivo RNAi screening identifies a mechanism of sorafenib resistance in liver cancer. Nature medicine 20: 1138-1146.

Ruiz i Altaba A, Mas C, Stecca B (2007). The Gli code: an information nexus regulating cell fate, stemness and cancer. Trends Cell Biol 17: 438-447.

Santos E, Tronick SR, Aaronson SA, Pulciani S, Barbacid M (1982). T24 human bladder carcinoma oncogene is an activated form of the normal human homologue of BALBand Harvey-MSV transforming genes. Nature 298: 343-347.

Scales SJ, de Sauvage FJ (2009). Mechanisms of Hedgehog pathway activation in cancer and implications for therapy. Trends in pharmacological sciences.

Schaaf G, Hamdi M, Zwijnenburg D, Lakeman A, Geerts D, Versteeg R et al (2010). Silencing of SPRY1 triggers complete regression of rhabdomyosarcoma tumors carrying a mutated RAS gene. Cancer research 70: 762-771.

Schindelin J, Arganda-Carreras I, Frise E, Kaynig V, Longair M, Pietzsch T et al (2012). Fiji: an open-source platform for biological-image analysis. Nature methods 9: 676-682.

Scholl FA, Betts DR, Niggli FK, Schafer BW (2000). Molecular features of a human rhabdomyosarcoma cell line with spontaneous metastatic progression. British journal of cancer 82: 1239-1245.

Schuler M, Ali F, Metzger E, Chambon P, Metzger D (2005). Temporally controlled targeted somatic mutagenesis in skeletal muscles of the mouse. Genesis 41: 165-170.

Serrano M, Lin AW, McCurrach ME, Beach D, Lowe SW (1997). Oncogenic ras provokes premature cell senescence associated with accumulation of p53 and p16INK4a. Cell 88: $593-602$. 
Seto M, Ohta M, Asaoka Y, Ikenoue T, Tada M, Miyabayashi K et al (2009). Regulation of the hedgehog signaling by the mitogen-activated protein kinase cascade in gastric cancer. Molecular carcinogenesis 48: 703-712.

Shern JF, Chen L, Chmielecki J, Wei JS, Patidar R, Rosenberg M et al (2014). Comprehensive genomic analysis of rhabdomyosarcoma reveals a landscape of alterations affecting a common genetic axis in fusion-positive and fusion-negative tumors. Cancer discovery 4: 216-231.

Shirokawa JM, Elisei R, Knauf JA, Hara T, Wang J, Saavedra HI et al (2000). Conditional apoptosis induced by oncogenic ras in thyroid cells. Mol Endocrinol 14: 1725-1738.

Shou Y, Robinson DM, Amakye DD, Rose KL, Cho YJ, Ligon KL et al (2015). A five-gene hedgehog signature developed as a patient preselection tool for hedgehog inhibitor therapy in medulloblastoma. Clinical cancer research : an official journal of the American Association for Cancer Research 21: 585-593.

Smith MA, Altekruse SF, Adamson PC, Reaman GH, Seibel NL (2014). Declining childhood and adolescent cancer mortality. Cancer 120: 2497-2506.

Soriano P (1999). Generalized lacZ expression with the ROSA26 Cre reporter strain. Nature genetics 21: 70-71.

St-Jacques B, Hammerschmidt M, McMahon AP (1999). Indian hedgehog signaling regulates proliferation and differentiation of chondrocytes and is essential for bone formation. Genes \& development 13: 2072-2086.

Stecca B, Mas C, Clement V, Zbinden M, Correa R, Piguet V et al (2007). Melanomas require HEDGEHOG-GLI signaling regulated by interactions between GLI1 and the RAS-MEK/AKT pathways. Proceedings of the National Academy of Sciences of the United States of America 104: 5895-5900.

Steelman LS, Pohnert SC, Shelton JG, Franklin RA, Bertrand FE, McCubrey JA (2004). JAK/STAT, Raf/MEK/ERK, PI3K/Akt and BCR-ABL in cell cycle progression and leukemogenesis. Leukemia 18: 189-218.

Stokoe D, Macdonald SG, Cadwallader K, Symons M, Hancock JF (1994). Activation of Raf as a result of recruitment to the plasma membrane. Science 264: 1463-1467.

Stratton MR, Fisher C, Gusterson BA, Cooper CS (1989). Detection of point mutations in Nras and K-ras genes of human embryonal rhabdomyosarcomas using oligonucleotide probes and the polymerase chain reaction. Cancer research 49: 6324-6327.

Sultan I, Qaddoumi I, Yaser S, Rodriguez-Galindo C, Ferrari A (2009). Comparing adult and pediatric rhabdomyosarcoma in the surveillance, epidemiology and end results program, 1973 to 2005: an analysis of 2,600 patients. Journal of clinical oncology : official journal of the American Society of Clinical Oncology 27: 3391-3397.

Tomayko MM, Reynolds CP (1989). Determination of subcutaneous tumor size in athymic (nude) mice. Cancer chemotherapy and pharmacology 24: 148-154. 
Tostar U, Malm CJ, Meis-Kindblom JM, Kindblom LG, Toftgard R, Unden AB (2006). Deregulation of the hedgehog signalling pathway: a possible role for the PTCH and SUFU genes in human rhabdomyoma and rhabdomyosarcoma development. J Pathol 208: $17-25$.

Tostar U, Toftgard R, Zaphiropoulos PG, Shimokawa T (2010). Reduction of human embryonal rhabdomyosarcoma tumor growth by inhibition of the hedgehog signaling pathway. Genes Cancer 1: 941-951.

Tsumura H, Yoshida T, Saito H, Imanaka-Yoshida K, Suzuki N (2006). Cooperation of oncogenic K-ras and p53 deficiency in pleomorphic rhabdomyosarcoma development in adult mice. Oncogene 25: 7673-7679.

Tuveson DA, Shaw AT, Willis NA, Silver DP, Jackson EL, Chang S et al (2004). Endogenous oncogenic K-ras(G12D) stimulates proliferation and widespread neoplastic and developmental defects. Cancer cell 5: 375-387.

Uhmann A, Ferch U, Bauer R, Tauber S, Arziman Z, Chen C et al (2005). A model for PTCH1/Ptch1-associated tumors comprising mutational inactivation and gene silencing. Int J Oncol 27: 1567-1575.

Uhmann A, Dittmann K, Nitzki F, Dressel R, Koleva M, Frommhold A et al (2007). The Hedgehog receptor Patched controls lymphoid lineage commitment. Blood 110: 18141823.

Uhmann A, Niemann H, Lammering B, Henkel C, Hess I, Nitzki F et al (2011). Antitumoral effects of calcitriol in basal cell carcinomas involve inhibition of hedgehog signaling and induction of vitamin D receptor signaling and differentiation. Molecular cancer therapeutics 10: 2179-2188.

Varjosalo M, Taipale J (2008). Hedgehog: functions and mechanisms. Genes \& development 22: $2454-2472$.

Wang B, Fallon JF, Beachy PA (2000). Hedgehog-regulated processing of Gli3 produces an anterior/posterior repressor gradient in the developing vertebrate limb. Cell 100: 423 434.

Wang C (2012). Childhood rhabdomyosarcoma: recent advances and prospective views. Journal of dental research 91: 341-350.

Wen X, Lai CK, Evangelista M, Hongo JA, de Sauvage FJ, Scales SJ (2010). Kinetics of hedgehog-dependent full-length Gli3 accumulation in primary cilia and subsequent degradation. Molecular and cellular biology 30: 1910-1922.

Wetmore C (2003). Sonic hedgehog in normal and neoplastic proliferation: insight gained from human tumors and animal models. Current opinion in genetics \& development 13: $34-42$.

Wexler L, LJ H (1997). Rhabdomyosarkoma and the Undifferentiated Sarcoma. In Principles and Practice of Pediatric Oncology; third edition, edited by Philip A Pizzo and David G Poplack Lippincott-Raven Publishers Philadelphia. 
Wohrle FU, Daly RJ, Brummer T (2009). Function, regulation and pathological roles of the Gab/DOS docking proteins. Cell communication and signaling : CCS 7: 22.

Yamanaka H, Oue T, Uehara S, Fukuzawa M (2011). Hedgehog signal inhibitor forskolin suppresses cell proliferation and tumor growth of human rhabdomyosarcoma xenograft. Journal of pediatric surgery 46: 320-325.

Yeh HH, Wu CH, Giri R, Kato K, Kohno K, Izumi H et al (2008). Oncogenic Ras-induced morphologic change is through MEK/ERK signaling pathway to downregulate Stat 3 at a posttranslational level in NIH3T3 cells. Neoplasia 10: 52-60.

Yu CF, Liu ZX, Cantley LG (2002). ERK negatively regulates the epidermal growth factormediated interaction of Gab1 and the phosphatidylinositol 3-kinase. The Journal of biological chemistry 277: 19382-19388.

Zheng CF, Guan KL (1993). Dephosphorylation and inactivation of the mitogen-activated protein kinase by a mitogen-induced Thr/Tyr protein phosphatase. The Journal of biological chemistry 268: 16116-16119.

Zibat A, Uhmann A, Nitzki F, Wijgerde M, Frommhold A, Heller T et al (2009). Time-point and dosage of gene inactivation determine the tumor spectrum in conditional Ptch knockouts. Carcinogenesis 30: 918-926.

Zibat A, Missiaglia E, Rosenberger A, Pritchard-Jones K, Shipley J, Hahn H et al (2010). Activation of the hedgehog pathway confers a poor prognosis in embryonal and fusion gene-negative alveolar rhabdomyosarcoma. Oncogene: Sep 6. [Epub ahead of print].

Zimmermann S, Moelling K (1999). Phosphorylation and regulation of Raf by Akt (protein kinase B). Science 286: 1741-1744. 


\section{Abbreviations}

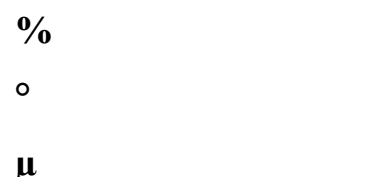

Ab

AEC

Akt/AKT

AP

ARMS

BCC

Bp

BrdU

BSA

cDNA

C

$\mathrm{CO}_{2}$

Cre

Ct

D

DAB

ddH2O

del

Dhh/DHH

DMEM

DMSO

Dnmt/DNMT

DNA

dNTPs

DTT

E

E

E. coli

EDTA

EGF

EGFR
Percent

Degree

Micro

Antibody

3-amino-9 ethylcarbazole

v-akt murine thymoma viral oncogene homolog or Protein kinase B

Alkaline phosphatase

Alveolar Rhabdomyosarcoma

Basal cell carcinoma

base pair

5-Bromo-2-Deoxyuridine

bovine serum albumine

cDNA copyDNA

Celcius

Carbon dioxyde

causes recombination

cycle threshold

Days

Diamino-Benzidine

double distilled water

deleted, deletion

Desert Hedgehog

Dulbeco's Modified Eagle Medium

N.N.-Dimethylsulfoxide

DNA methyltransferase

Deoxyribonucleic acid

Desoxyribonucleotide triphosphate

Dithiothreitol

Extinction

Exon

Escherichia coli

Ethylen-diamine-tetraacetate

epidermal growth factor

endothelial growth factor receptor 


\begin{tabular}{|c|c|}
\hline ERMS & Embryonal Rhabdomyosarcoma \\
\hline ERT & tamoxifen-inducible estrogen receptor \\
\hline EtOH & ethanol \\
\hline FCS & fetal calf serum \\
\hline FICS & Fluorescein isothiocyanate \\
\hline FoxF1/FOXF1 & Forkhead box F1 \\
\hline GAPs & GTPase Activating Protein \\
\hline gDNA & genomic DNA \\
\hline GDP & Guanosine diphosphate \\
\hline GDS & Guanine Dissociation Stimulators \\
\hline GEF & Guanine nucleotide Exchange Factor \\
\hline Gli/GLI & Glioma oncogene \\
\hline $\mathbf{G l i}_{\text {act }}$ & Gli activator form \\
\hline $\mathbf{G l i}_{\text {rep }}$ & Gli repressor form \\
\hline GTP & Guanosine triphosphate \\
\hline Erk/ERK & Extracellular-signal-regulated kinases \\
\hline $\mathbf{H}$ & hour(s) \\
\hline $\mathbf{H}_{2} \mathbf{O}_{2}$ & hydrogen peroxide \\
\hline $\mathrm{HCl}$ & hydrochloric acid \\
\hline $\mathbf{H} \& \mathbf{E}$ & Haematoxylin/Eosin \\
\hline HEK-293 & human embryonic kidney cells 293 \\
\hline Hh/HH & Hedgehog \\
\hline HhA & $\begin{array}{l}\text { HhAntag: (Benzamide, N-[4-chloro-3-[6-(dimethylamino)-1H- } \\
\text { benzimidazol-2-yl]phenyl]-3,5-dimethoxy-) }\end{array}$ \\
\hline HRP & horseradish peroxidase \\
\hline Hsc70 & heat-shock protein 70 \\
\hline i.p. & Intraperitoneal \\
\hline IHC & Immunhistochemistry \\
\hline Ihh/IHH & Indian Hedgehog \\
\hline $\mathbf{K}$ & Kilo \\
\hline kDa & Kilodalton \\
\hline LacZ & LacZ $\beta$-Galactosidase \\
\hline LB-Medium & lysogeny broth Medium \\
\hline $\operatorname{loxP}$ & $\begin{array}{l}\text { loxP Sequence (DNA-Consensus sequence for binding of cre- } \\
\text { recombinase) }\end{array}$ \\
\hline Map & Mitogen-activated protein \\
\hline
\end{tabular}


Mek/MEK

Min

MSCV

MTOR

mRNA

Myf5

N

neoR

NF1

Nf-kB

n.s.

N-terminal

OD

OIS

PBS

PCR

PFA

pH

PI103

PI3K

$\mathrm{PIP}_{2}$

$\mathrm{PIP}_{3}$

PS

Ptch

Ptch $^{\text {del }}$

PDK1

p53

qRT-PCR

Raf/RAF

Ras/RAS

RIPA buffer

RLU

RMS

RNA

Rpm

RT
MAP kinase-extracellular signal-regulated kinase

minute(s)

murine stem cell virus

mammalian target of rapamycin

messenger RNA

Myogenic factor 5

number (sample)

Neomycin resistance

Neurofibromin 1

kappa light polypeptide gene enhancer in B cells

not significant

$\mathrm{N}$-terminal aminoterminal

optical density

oncogene-induced senescence

Phosphate buffered saline

Polymerase chain reaction

Paraformaldehyde

lat. potentia hydrogenii

PI3-K alpha Inhibitor 1

Phosphatidylinositol 3-kinase

Phosphatidylinositol (4,5)-bisphosphate

Phosphatidylinositol $(3,4,5)$-trisphosphate

Penicillin/Streptomycin

Patched

Ptch allele with deletion of Exon 8 and 9

Phosphotidylinositide-dependent kinase

Transformation related protein 53

quantitative real time PCR

rapidly accelerated fibrosarcoma

Rat sarcoma

radioimmunoprecipitation assay buffer

relative light units

Rhabdomyosarcoma

Ribonucleicacid

rounds per minute

room temperature 
RTK

Stat3

SDS

SEM

Shh/SHH

SMA

Smo/SMO

SNP

SOC

STE

Sufu

SYBR.

T

Taq

TBE

TBS

TBST

Tris

U

UO126

$\mathbf{v} / \mathbf{v}$

Vol

$\mathbf{w} / \mathbf{v}$

WST-1

wt

X-Gal
Receptor tyrosine kinase

signal transducer and activator of transcription

Sodiumdodecyl sulfate

standard error of the mean

Sonic hedgehog

Smooth muscle actin

Smoothened

single nucleotide polymorphism

super optimal broth with catabolite repression

SDS/Tris/EDTA-buffer

Suppressor of fused

Synergy Brands Inc

transgenes Allele

Thermus aquaticus

Tris-boric acid-EDTA-solution

Tris-buffered saline

Tris-buffered sodium chloride-solution containing Tween-20

tris(hydroxymethyl)aminomethane

unit (enzyme)

1,4-Diamino-2,3-dicyano-1,4-bis[2-aminophenylthio]butadiene

volume/volume

Volume

weight/volume

water soluble tetrazolium salt 1

Wild type

5-Bromo-4-chloro-3-indolyl- $\beta$-Galaktosid 


\section{Appendix}

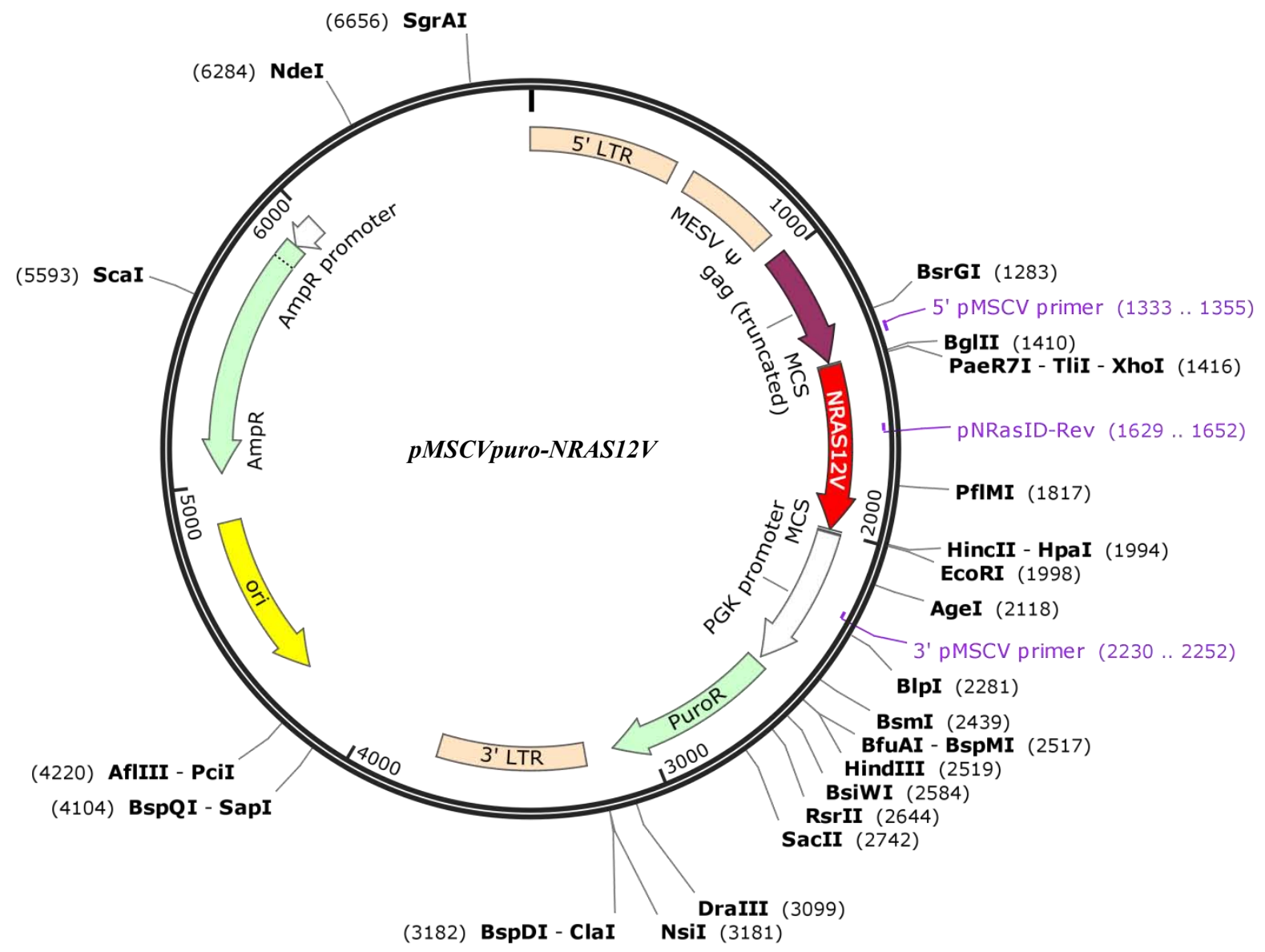

Appendix 1: Plasmid map of pMSCVpuro-NRAS12V plasmid. The plasmid was used to stably generate constitutive active RAS signaling in human RMS cell lines. The map was created by using SnapGene ${ }^{\circledR}$ Viewer. 


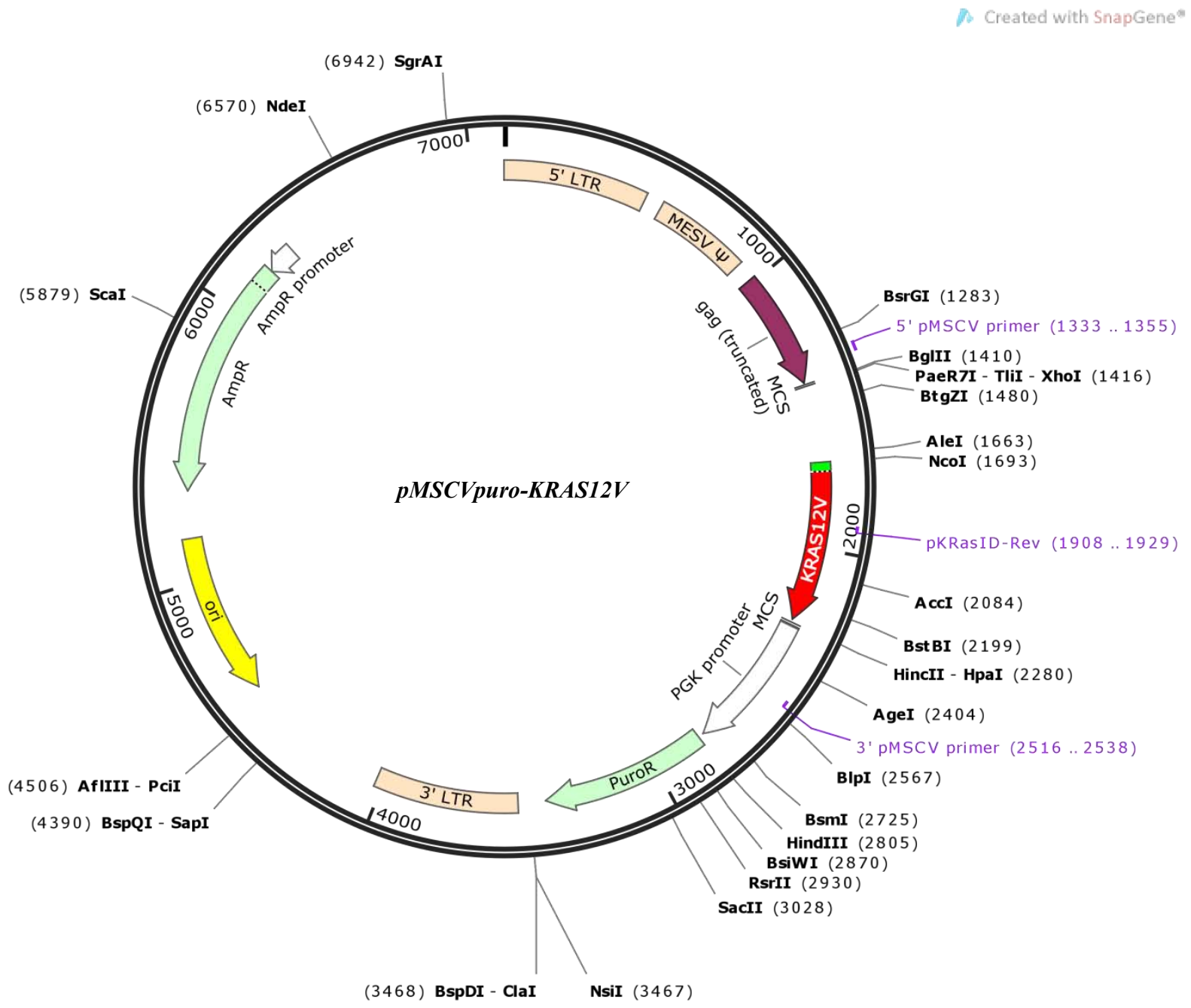

Appendix 2: Plasmid map of pMSCVpuro-KRAS12V plasmid. The plasmid was used to stably generate constitutive active KRAS signaling in human RMS cell lines. The map was created by using SnapGene ${ }^{\circledR}$ Viewer. 


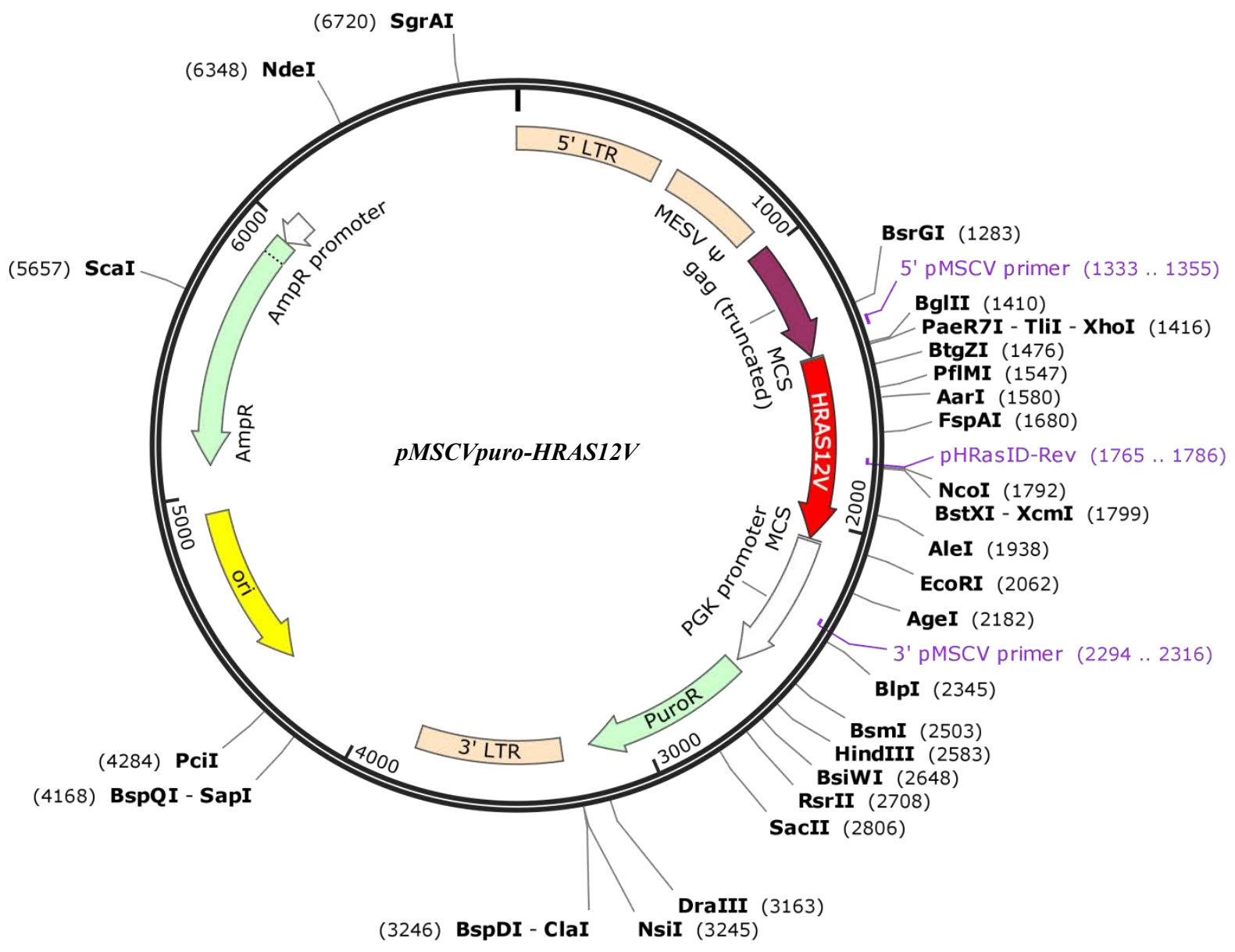

Appendix 3: Plasmid map of pMSCVpuro-HRAS12V plasmid. The plasmid was used to stably generate constitutive active HRAS signaling in human RMS cell lines. The map was created by using SnapGene ${ }^{\circledR}$ Viewer. 


\section{Acknowledgement}

This thesis would not have been possible without the support, the good feedbacks and help of the people around me. Especially, my major thanks go to Prof. Dr. Heidi Hahn for given me the opportunity to work in her working group, for her endless support, for her always open door and the inspiring discussions. Without you I cannot have finished this work. Thank you Heidi!

Furthermore I would also like to thank the members of my thesis committee meetings Prof. Dr. Dieter Kube and Prof. Dr. Felix Brembeck for the helpful inputs and exciting discussions during the meetings.

Special thanks go to the "mouse house girls" Susan Peter and Jennifer Flemming for helping me with words and deeds, for the welcoming atmosphere and all the phone calls about the state of my mice. Remain as you are, you are great!

A big thank you goes to Dr. Michael Engelke and Prof Dr. Tobias Pukrop for helping me to develop the stable transduced cells.

Of course, very special thanks go to the actual and former members of the lab for the exchange, the tireless support, the scientific discussions and the barbecues after work. Thank you, Dr. Anja Uhmann, Dr. Frauke Nitzki, Dr. Penelope Pelczar, Dr. Benedikt Linder, Dr. Marco Becker, Dr. Rosalie Ridzewski, Julia Dräger, Joana Pycek, Natalie Hönig and Julia Heise. Furthermore I would like to thank Anke Fromhold and Ina Hess for their technical support. Thank you for the trivial "Lab-mess".

And most importantly my sincere appreciation and heartfelt thanks goes to Michael, my lovely partner, thank you for helping me through the ups and downs during this phase of my live, for supporting me, helping me to come down and for cheering me up when I needed it. Thank you very much!

And at the end I would like to say a special thanks to my parents Regina and Hans Peter. They have supported me in a grand manner and given me the best starting conditions for my way. Without you I would not be here. Thank you! 


\section{Curriculum vitae}

\begin{tabular}{|c|c|}
\hline 012-hitherio & $\mathrm{PhD}$ in Biology \\
\hline & Georg-August University of Göttingen, Germany \\
\hline & Georg-August University School of Science (GAUSS) \\
\hline & Program: Molecular Medicine \\
\hline & $\begin{array}{ll}\text { Title of the Thesis: Interaction between Hedgehog/Ptched and Ras signaling } \\
\text { in Rhabdomyosarcoma }\end{array}$ \\
\hline 2009-2011 & Master of Science in Biomedical Sciences \\
\hline & Bonn-Rhein-Sieg University \\
\hline & Applies Sciences \\
\hline & Molecular biology / genetics, immunology, human biology and physiology \\
\hline & $\begin{array}{l}\text { Title of the Thesis: Functional characterization of colchicine analogues as } \\
\text { target against lymphoma }\end{array}$ \\
\hline 2006-2009 & Bachelor of Science in Applied Biology \\
\hline & Bonn-Rhein-Sieg University \\
\hline & Applies Sciences \\
\hline & Human biology, Microbiology, genetic und cell culture \\
\hline & $\begin{array}{ll}\text { Title of the Thesis: } & \text { Construction of a recombinant C.glutamicum strain that } \\
& \text { allows constitutive production of poly(3- } \\
& \text { hydroxybuturate) }\end{array}$ \\
\hline
\end{tabular}

\section{Research Publications}

Nitzki F, Cuvelier N, Drager J, Schneider A, Braun T, Hahn H (2015). Hedgehog/Patchedassociated rhabdomyosarcoma formation from deltal-expressing mesodermal cells. Oncogene.

Ridzewski R, Rettberg D, Dittmann K, Cuvelier N, Fulda S, Hahn H (2015). Hedgehog Inhibitors in Rhabdomyosarcoma: A Comparison of Four Compounds and Responsiveness of Four Cell Lines. Frontiers in oncology 5: 130.

Schott C, Graab U, Cuvelier N, Hahn H, Fulda S (2015). Oncogenic RAS Mutants Confer Resistance of RMS13 Rhabdomyosarcoma Cells to Oxidative Stress-Induced Ferroptotic Cell Death. Frontiers in oncology 5: 131.

Nitzki F, Tolosa EJ, Cuvelier N, Frommhold A, Salinas-Riester G, Johnsen SA et al (2015). Overexpression of mutant Ptch in rhabdomyosarcomas is associated with promoter hypomethylation and increased Gli1 and H3K4me3 occupancy. Oncotarget 6: 9113-9124.

Sitnikov N, Velder J, Abodo L, Cuvelier N, Neudorfl J, Prokop A et al (2012). Total synthesis of indole-derived allocolchicine analogues exhibiting strong apoptosis-inducing activity. Chemistry 18: 12096-12102. 